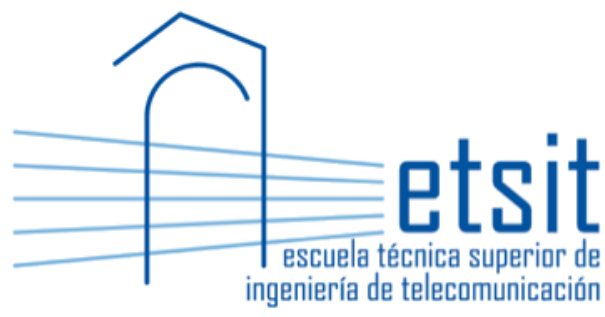

\title{
Análisis Experimental de la Propagación en Redes de Área Corporal para la Banda de Ultra Wideband
}

Autor:

Rubén Gregorio García Serna

Directores:

Dr. D. José María Molina García-Pardo

Dra. D. ${ }^{a}$ Concepción García Pardo 



\section{Universidad \\ Politécnica \\ de Cartagena}

DT-16

\section{CONFORMIDAD DE SOLICITUD DEAUTORIZACIÓN DE DEPÓSITO DE TESIS DOCTORAL POR EL/LA DIRECTOR/A DE LA TESIS}

D. José María Molina García-Pardo y Da . Concepción García Pardo, Director y Codirectora de la Tesis doctoral "Análisis Experimental de la Propagación en Redes de Área Corporal para la Banda de Ultra Wideband".

\section{INFORMAN:}

Que la referida Tesis Doctoral, ha sido realizada por D. Rubén Gregorio García Serna, dentro del Programa de Doctorado Tecnologías de la Información y las Comunicaciones, dando nuestra conformidad para que sea presentada ante el Comité de Dirección de la Escuela Internacional de Doctorado para ser autorizado su depósito.

La rama de conocimiento en la que esta tesis ha sido desarrollada es:

․ Ciencias

․ Ciencias Sociales y Jurídicas

Ingeniería y Arquitectura

En Cartagena, a 4 de ABRIL de 2017

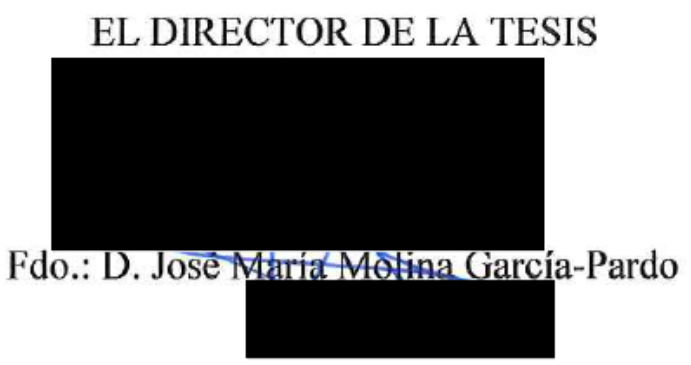

LA CODIRECTORA DE LA TESIS

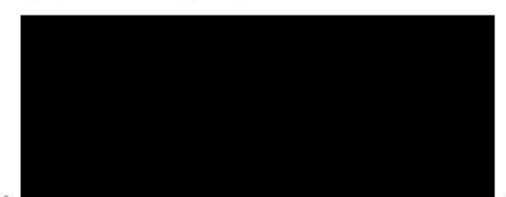

Fdo.: D". Concepcron Garcla Yardo 


\section{Universidad \\ Politécnica \\ de Cartagena}

DT-17

\section{CONFORMIIDAD DE DEPÓSITO DE TESIS DOCTORAL POR LA COMISIÓN ACADÉMICA DEL PROGRAMA}

D. Fernando Daniel Quesada Pereira, Presidente de la Comisión Académica del Programa Tecnologías de la Información y las Comunicaciones.

\section{INFORMA:}

Que la Tesis Doctoral titulada, "Análisis Experimental de la Propagación en Redes de Área Corporal para la Banda de Ultra Wideband", ha sido realizada, dentro del mencionado Programa de Doctorado, por D. Rubén Gregorio García Serna, bajo la dirección y supervisión del Dr. José María Molina García-Pardo y la Dra. Concepción García Pardo.

En reunión de la Comisión Académica de fecha $04 / 04 / 12$, visto que en la misma se acreditan los indicios de calidad correspondientes y la autorización del Director/a de la misma, se acordó dar la conformidad, con la finalidad de que sea autorizado su depósito por el Comité de Dirección de la Escuela Internacional de Doctorado.

La Rama de conocimiento por la que esta tesis ha sido desarrollada es:

$\square$ Ciencias

$\square \quad$ Ciencias Sociales y Jurídicas

$\not$ Ingeniería y Arquitectura

En Cartagena, a 4 de $A B R / L$ de 2017

EL PRESIDENTE DE LA_GQMISIÓN ACADÉMICA

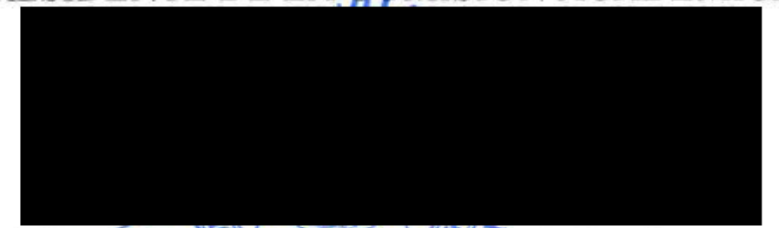

Fdo. D. Fernando Daniel ouesada Pereira $\frac{\cos 20}{\cos 2}$ 


\section{AGRADECIMIENTOS}

Desarrollar una tesis doctoral es un proceso complejo que implica muchos sacrificios a nivel personal. Durante todo este tiempo son muchas las personas que me han animado a seguir adelante, por lo que realmente me parece casi imposible dar las gracias a todos los que, de alguna forma, me han apoyado a lo largo de este recorrido.

En primer lugar, me gustaría dar las gracias a mis directores de tesis José María Molina García-Pardo y Concepción García Pardo por darme la oportunidad de desarrollar este proyecto con ellos y por sus consejos durante todo este tiempo.

En segundo lugar, quiero agradecer a mis padres Encarna y Onofre por todo el cariño y ánimo que me han dado durante estos años. También quiero agradecer a mis abuelos Encarna, Amador y Pepita por tener la total y absoluta confianza de que terminaría esta etapa en algún momento.

Gracias a mi hermano David y el resto de mi familia por echarme una mano siempre que lo he necesitado.

Por último, tengo que darte las gracias a ti Noelia, por estar a mi lado, por apoyarme, por soportarme y por creer en mi. 


\section{RESUMEN}

La posibilidad de monitorizar la actividad del cuerpo humano, ya sea desde la perspectiva médica, del deporte o del entretenimiento, utilizando dispositivos de diversa índole desplegados en el interior o sobre el cuerpo es en la actualidad un tema que está despertando un enorme interés tanto en el ámbito científico como social. Desde implantes inteligentes a elementos capaces de registrar nuestras constantes vitales o cuantificar nuestra actividad física, multitud de dispositivos son susceptibles de organizarse formando una red de elementos interconectados de manera inalámbrica con el objetivo de transmitir la información que sus respectivos sensores han recogido. A esta estructura de red centrada en el cuerpo se le denomina de manera genérica red de área corporal inalámbrica (WBAN, Wireless Body Area Network).

La interacción con el cuerpo hace que la propagación de señales entre dispositivos de una WBAN presente características diferenciadoras respecto a las encontradas en otros canales radio tradicionales. Además, debido a que los tejidos biológicos presentan características dieléctricas (permitividad relativa y conductividad) dependientes de la frecuencia, el diseño de sistemas que operen de manera eficiente en este entorno requiere el desarrollo de modelos que describan, según la frecuencia de operación, los fenómenos que afectan a la propagación en los distintos canales radio entre dispositivos ubicados en el interior, la superficie o en la proximidad del cuerpo.

Entre las diferentes bandas de frecuencias propuestas para redes WBAN, la banda de Ultra Wideband (UWB) de $3.1 \mathrm{GHz}$ a $10.6 \mathrm{GHz}$ ha captado un gran interés en los últimos años debido a que, características tales como el alto ancho de banda, baja potencia de emisión, alto nivel de seguridad, reducidas dimensiones de los dispositivos y alta resolución temporal, la hacen especialmente adecuada en su aplicación a este tipo de redes.

Con el objetivo de caracterizar la propagación entre dispositivos de una WBAN operando a frecuencias dentro de la banda de UWB, la presente tesis recoge los resultados de los estudios realizados, destinados por un lado a la caracterización del canal de propagación off-body entre un dispositivo colocado sobre la superficie del cuerpo y un punto de acceso externo considerando el canal estacionario en el tiempo (o estático) y condiciones de visión directa, y por otro lado la caracterización de los efectos sobre el canal radio derivados del 
movimiento relativo entre dos dispositivos en una WBAN debido a la respiración, considerando al menos uno de ellos ubicado en el interior del cuerpo. En el primer caso el canal off-body estático se ha modelado a partir de las medidas sobre sujetos reales considerando diferentes posiciones de colocación de una antena receptora sobre el cuerpo y dos posturas: de pie y tumbado. En el segundo caso, el canal de propagación in-body se ha modelado empleando un phantom líquido para emular las condiciones de propagación en el interior del cuerpo en la banda de UWB y se han analizado los escenarios de propagación correspondientes a considerar un dispositivo en el interior del cuerpo y otro ubicado sobre la superficie del mismo (canal in-body a on-body), fuera de este a una cierta distancia (canal inbody a off-body) y, también en el interior conjuntamente con el primer dispositivo (canal inbody a in-body). 


\section{ABSTRACT}

Monitoring the body activity from the health, sport or entertainment point of view, by means of smart devices deployed in, on or around the human body is one of the most appealing topics from the last years in the scientific research and social areas. From smart implants to electronic devices designed to register our vital signs or to quantify our every day activity, a plethora of devices with sensing capabilities can be able to arrange into an interconnected wireless network topology in order to transmit the collected data. This kind of network where the human (or even animal) body is the main interaction element is called wireless body area network (WBAN).

The electromagnetic interaction among the devices and the body makes the signal propagation phenomenon in a WBAN exhibits unique characteristics compared to the ones shown in traditional radio channels. Due to the frequency dependence of the dielectrical properties (relative permittivity and conductivity) of biological tissues, there is a need of new models describing the propagation among network nodes placed inside the human body, on the surface or in an external (around the body) position at several frequencies.

From the group of proposed frequency bands to establish wireless connections among nodes in a WBAN, the Ultra Wideband (UWB) band from $3.1 \mathrm{GHz}$ to $10.6 \mathrm{GHz}$ is getting great attention during the last years because special characteristics like the high available bandwidth, low transmission power, high security level, low profile devices and high temporal and spatial resolution, just to name a few, make this band particularly appropriate to this kind of networks.

With the aim of fulfilling the lack of models describing the UWB radio channel in WBAN, this thesis presents the results of the experimental channel characterization research activity performed on the one hand, from the time invariant (or static) off-body channel point of view between a node placed on the body surface and an external access point considering line of sight conditions, and from the other hand, from the dynamic point of view considering the effects of the relative movement between two WBAN nodes due to breathing on the radio channel, considering at least one of them placed inside the human body. The off-body radio channel has been modeled from the measurements performed on real subjects considering different attachment positions of a receiver antenna on the body and 
two body postures: standing and lying down. The dynamic channel has been modeled using a liquid phantom to emulate the propagation inside the body at UWB frequencies and the propagation channel has been studied considering one device inside the body and another placed on the body surface (in-body to on-body channel), off the body at some separation distances (in-body to off-body channel) and both devices inside the body (in-body to in-body channel). 


\section{CONTENIDOS}

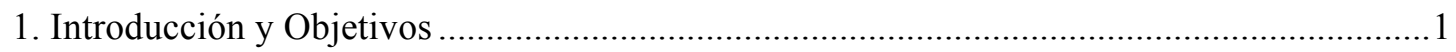

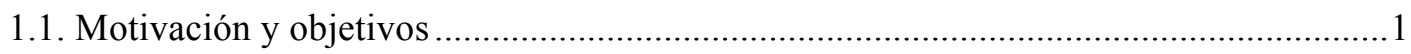

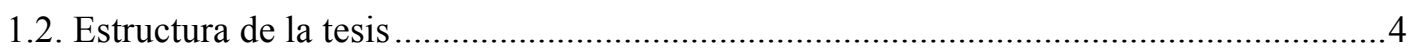

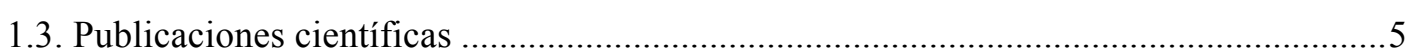

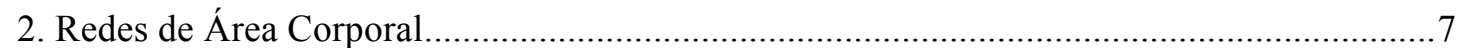

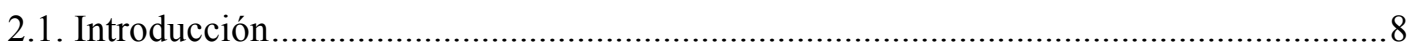

2.2. Tecnologías inalámbricas en redes de área corporal ...............................................10

2.3. Estandarización en WBAN. El estándar IEEE 802.15.6 …..........................................13

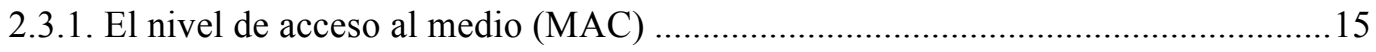

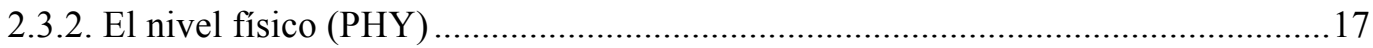

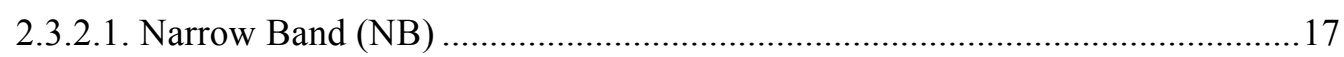

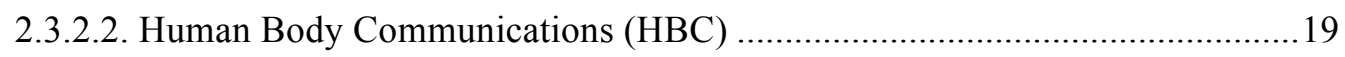

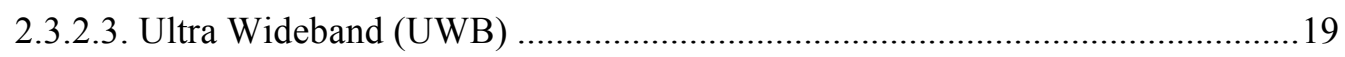

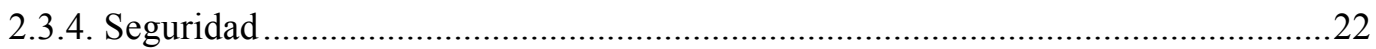

2.4. Introducción a la tecnología Ultra Wideband (UWB) ..................................................22

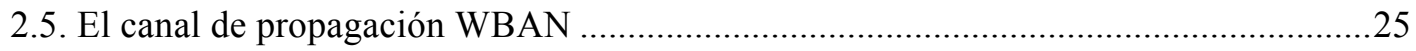

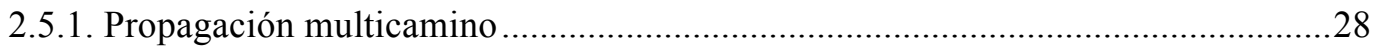

2.5.2. Modelado del canal de propagación WBAN en UWB...........................................30

2.5.2.1. Modelado de las pérdidas de propagación.........................................................30

2.5.2.2. Modelado del perfil de potencia-retardo (PDP) ...............................................33

2.5.3. Modelos estadísticos de canal WBAN en UWB …………………………..............34

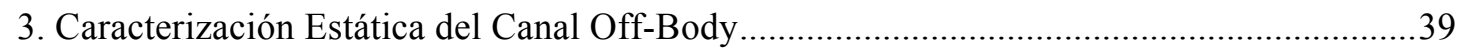

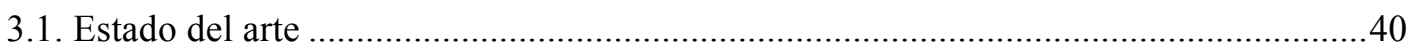

3.2. Esquema de medidas del canal de propagación off-body estático ................................43

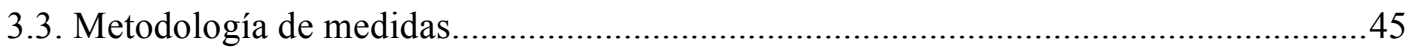

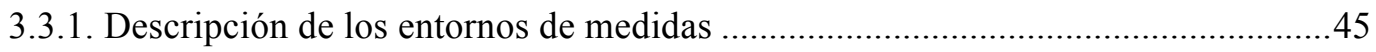

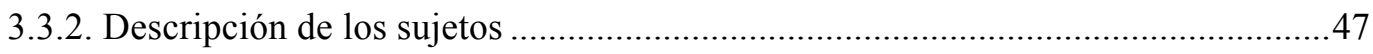

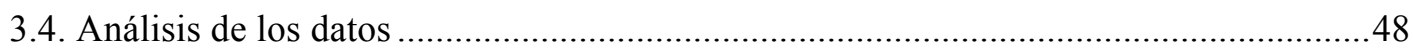

3.5. Influencia del entorno de medidas en el canal de propagación off-body UWB .............51

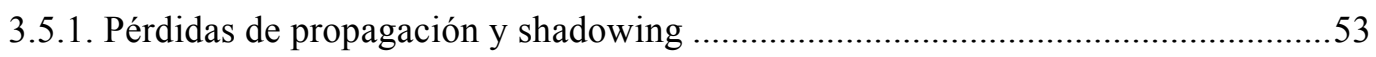

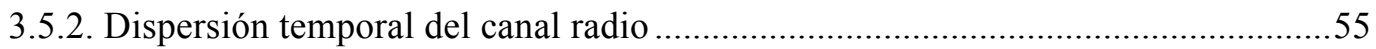


3.6. Influencia de la posición de colocación de una antena sobre el cuerpo en el canal off-

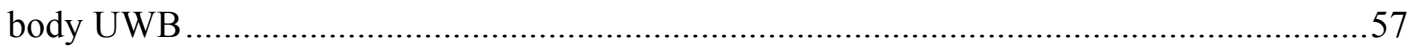

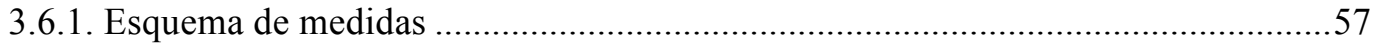

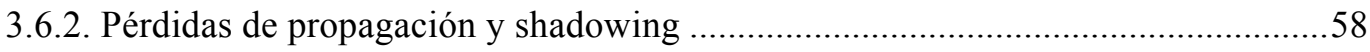

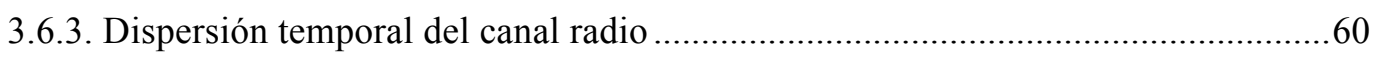

3.7. Influencia de la postura en el canal de propagación off-body UWB ............................62

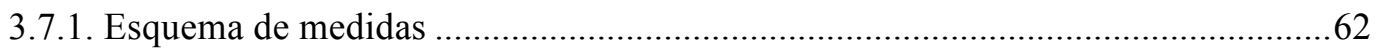

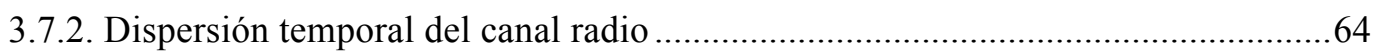

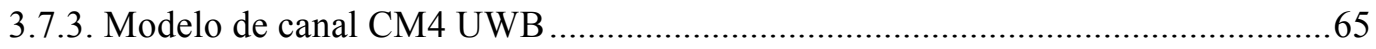

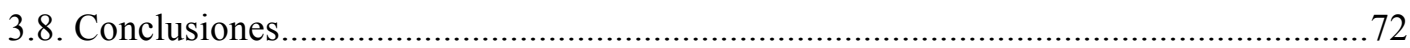

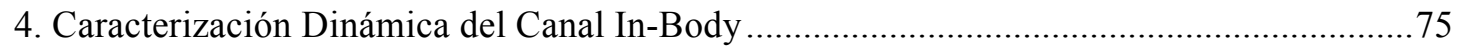

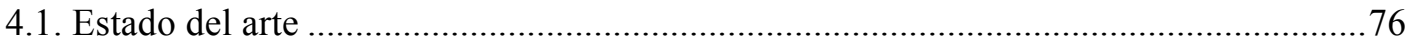

4.2. Esquema de medidas del canal de propagación in-body en condiciones dinámicas .....79

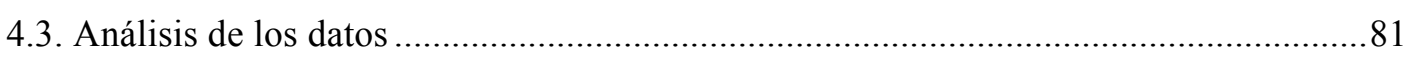

4.3.1. Caracterización del movimiento relativo entre dispositivos .................................82

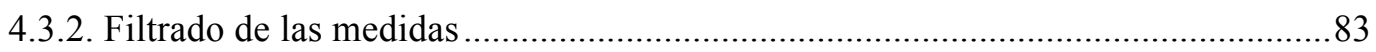

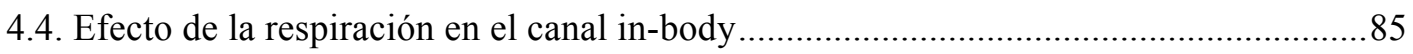

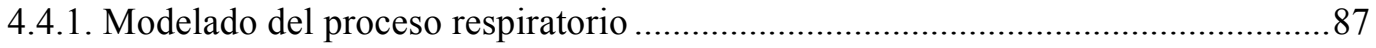

4.4.1.1. Modelado del proceso respiratorio para el canal IB2OB ...............................89

4.4.1.2. Modelado del proceso respiratorio para el canal IB2OFF.............................91

4.4.1.3. Modelado del proceso respiratorio para el canal IB2IB .................................93

4.5. Caracterización en frecuencia del canal in-body dinámico ...........................................95

4.5.1. Modelado de la forma del espectro Doppler ......................................................96

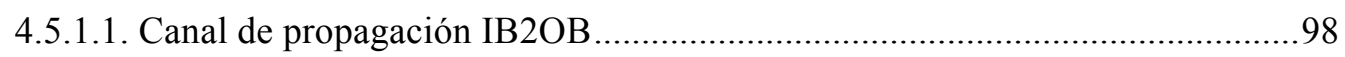

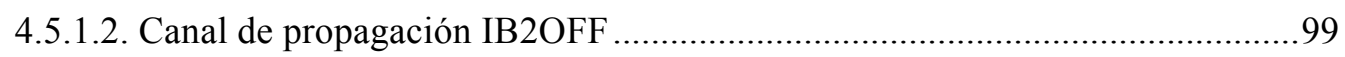

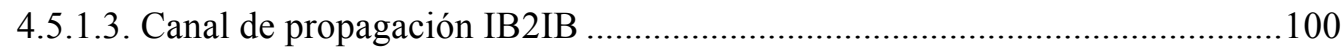

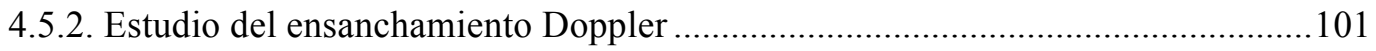

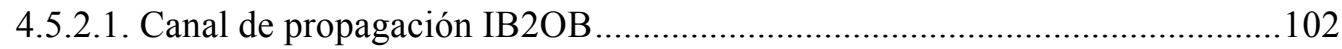

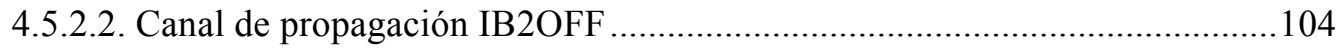

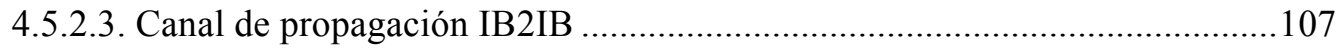

4.6. Caracterización en el tiempo del canal in-body dinámico.............................................109

4.6.1. Caracterización de la autocorrelación en el canal IB2OB....................................111

4.6.2. Caracterización de la autocorrelación en el canal IB2OFF ..................................112

4.6.3. Caracterización de la autocorrelación en el canal IB2IB ....................................113

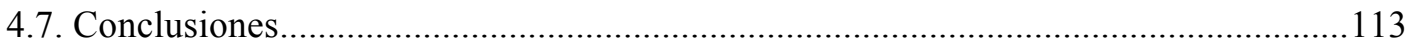

5. Conclusiones y Futuras Líneas de Investigación..............................................................117 
5.1. Conclusiones. 118

5.2. Futuras líneas de investigación 120

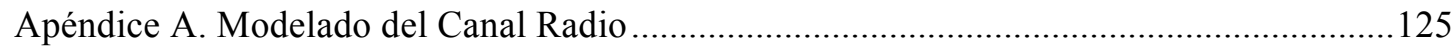

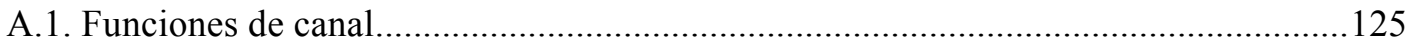

A.1.1. Canales deterministas variables en el tiempo .....................................................126

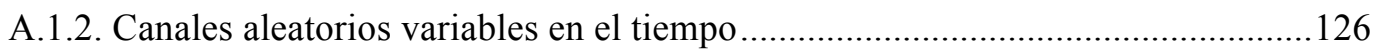

A.1.3. Clasificación de canales habituales. Simplificaciones sobre las funciones de

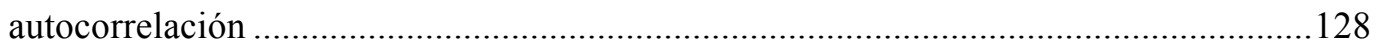

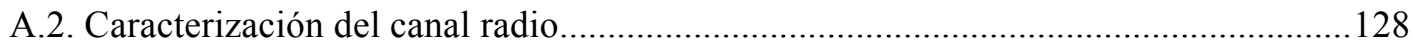

A.2.1. Modelado estático del canal radio ....................................................................129

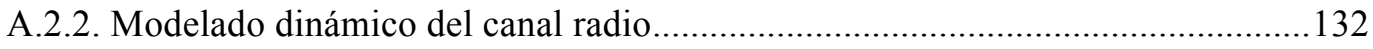

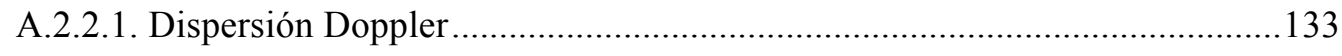

A.2.2.2. Tiempo de coherencia del canal .................................................................. 134

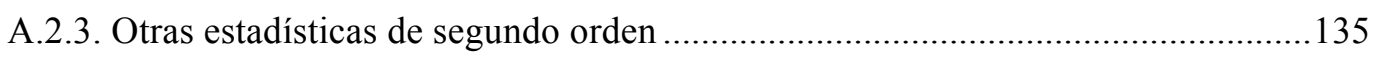

A.2.4. Metodología de modelado del canal de propagación ..........................................136

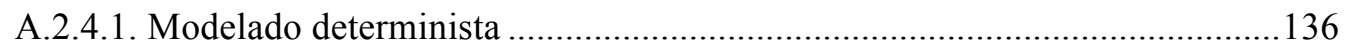

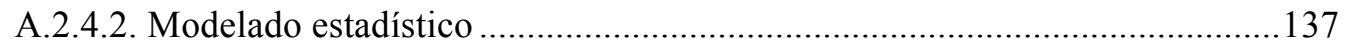

Apéndice B. Fundamentos de la Propagación en Tejidos Biológicos....................................141

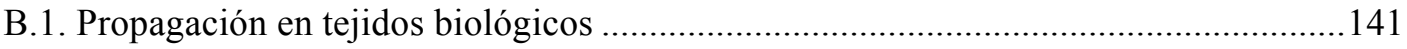

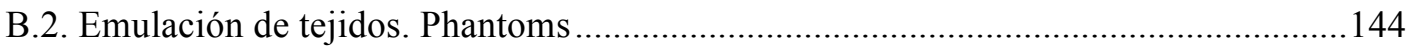

B.2.1. Modelos numéricos (phantoms numéricos) ......................................................145

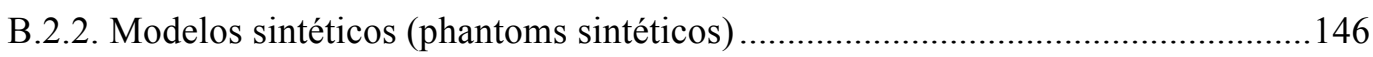

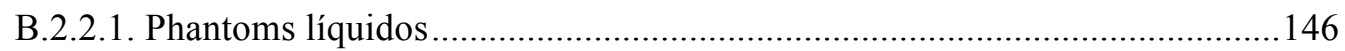

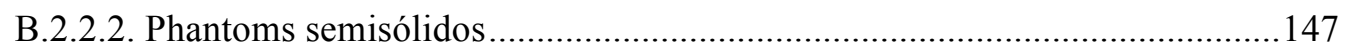

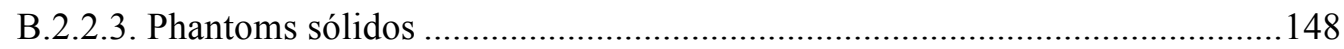

Apéndice C. Hardware y Software para Medidas del Canal Radio .......................................149

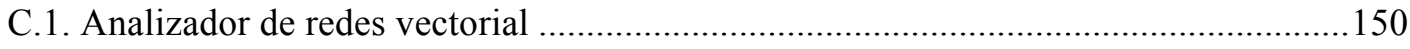

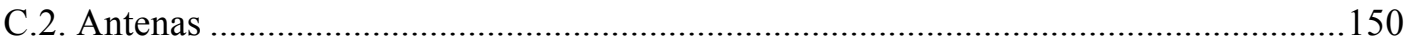

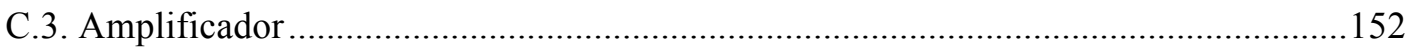

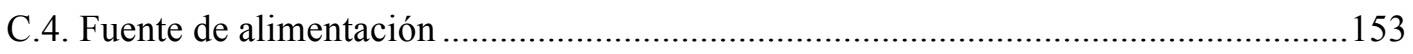

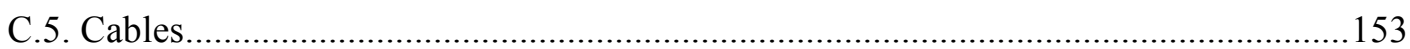

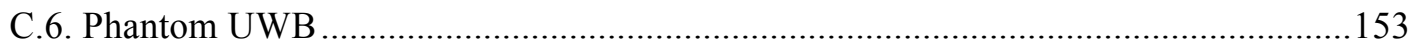

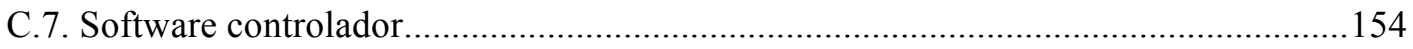

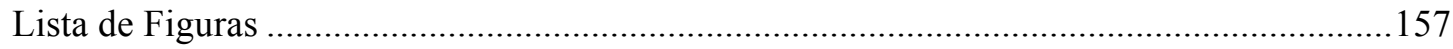

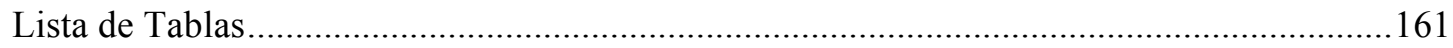




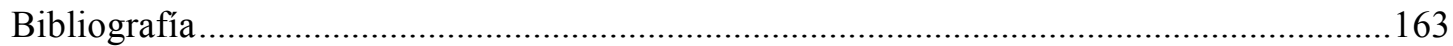

Acrónimos 175 


\section{Introducción y Objetivos}

\subsection{Motivación y objetivos}

Como consecuencia del aumento en la esperanza de vida y la disminución de la natalidad en la mayoría de las sociedades, la edad media de la población mundial se encuentra en constante aumento. Entre 2015 y 2050 la proporción de la población mundial con más de 60 años de edad pasará de cerca de 900 millones hasta los 2000 millones lo cual, teniendo en cuenta que la población mundial a fecha del 2015 era de 7300 millones de personas y en el 2050 se espera que supere los 9300 millones, representa un aumento del $12 \%$ al $22 \%$ y, en particular en Europa, este aumento se elevará hasta el 34\% para el 2050 [UN, 2015]. En la Figura 1.1 se presenta la evolución prevista en el número de individuos por sexo en Europa entre 2015 y 2050.
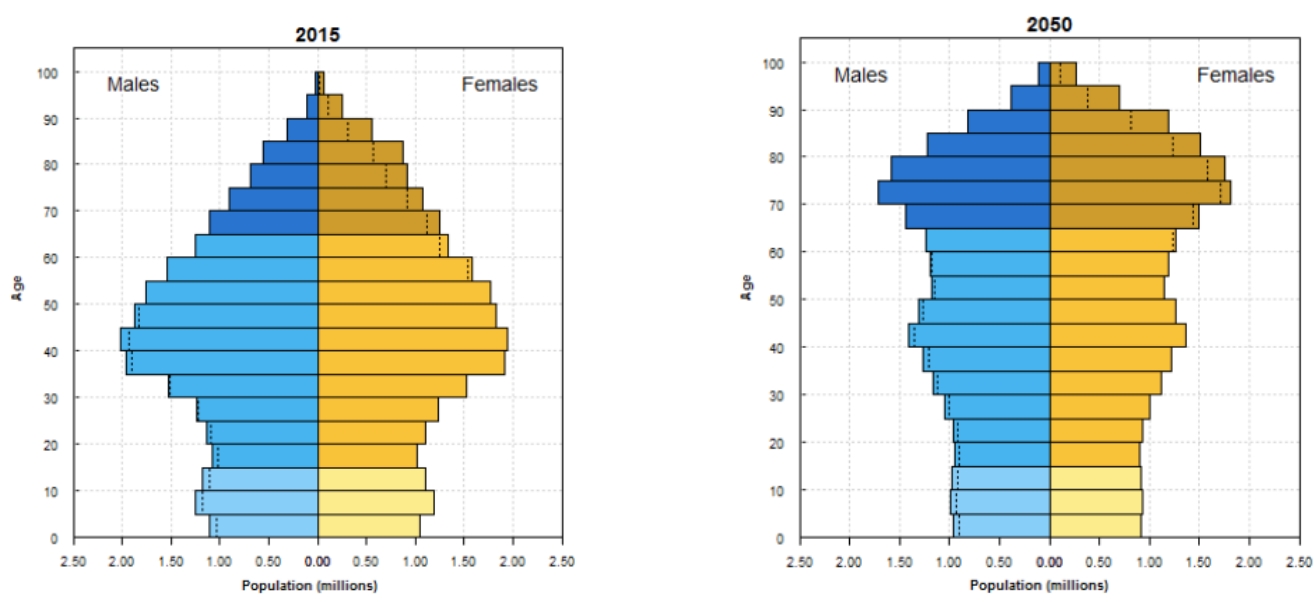

Figura 1.1: Distribución de la población en Europa según edad media [UN, 2015].

El envejecimiento progresivo de la población presenta serios retos económicos debido a los costes asociados a las enfermedades crónicas y la escasez de recursos sanitarios (personal médico, hospitales, camas, etc.). Esto conlleva que el desarrollo sostenible de las sociedades pase por encontrar medidas que optimicen las infraestructuras sanitarias existentes buscando soluciones tecnológicas que minimicen el impacto del incremento de la edad media atendiendo a dos premisas: prevención y atención remota. 
Las políticas de envejecimiento activo [OMS, 2015] y la implantación masiva de sistemas que ayuden a las personas a mantener un estado óptimo de salud hasta el máximo de edad posible constituyen los elementos centrales de la prevención. Al aumentar la calidad de vida de las personas durante más años, estas pueden seguir siendo miembros activos de la sociedad aportando valor a la economía. Por otro lado, la optimización de los recursos sanitarios pasa por la utilización de sistemas que permitan monitorizar la salud de los pacientes de manera remota, en los casos en los sea posible, lo cual permite seguir la evolución del estado de salud de los pacientes casi en tiempo real y tomar medidas de urgencia efectivas en caso de incidencias graves. Esto constituye un cambio de paradigma, migrando del modelo actual basado en la atención sanitaria reactiva, en el cual no se toman acciones sobre la salud de un paciente hasta que este no busca ayuda, a un modelo activo en el cual se puedan tomar acciones previo a que el propio paciente muestre síntomas [Gupta et al., 2013].

Durante los últimos años estamos asistiendo a un desarrollo sin precedentes en el área de la electrónica y las comunicaciones. Hoy en día vemos como habitual aquello que nos parecía imposible hace solo unos años $\mathrm{y}$, sin lugar a duda, es un proceso sin vuelta atrás. Los avances en las técnicas de fabricación y los materiales nos han permitido disponer en tamaños cada vez más reducidos, de dispositivos dotados de sensores capaces de medir determinados aspectos de la realidad física en la que se encuentran. De manera aislada, esta característica ofrece únicamente una visión limitada de un aspecto del proceso medido, pero de manera conjunta permite obtener una perspectiva general y tomar decisiones en base a una cierta lógica. Múltiples dispositivos organizados y ubicados, ya sea en el interior del cuerpo, sobre la superficie o en su inmediata proximidad, pueden obtener información acerca de parámetros fisiológicos, cinemáticos o contextuales del mismo y transmitirlos de manera inalámbrica hacia otro elemento capaz de realizar las funciones de concentrador (hub) y re-transmisor. A esta estructura se le denomina red de área corporal inalámbrica (WBAN, Wireless Body Area Network). Los datos recogidos pueden tener utilidad en un ámbito local o personal, o bien pueden reenviarse hacia un sistema remoto capaz de almacenar de manera segura esta información y proporcionar mecanismos para procesar los datos con propósitos específicos, como la monitorización médica, o integrarlos en una estructura organizativa de nivel superior aportando valor a una Ciudad/Región Inteligente (Smart City/Region).

Con el fin de promover la implantación masiva de las WBAN y como respuesta a las limitaciones que los anteriores estándares para redes de área personal (PAN, Personal Area Network), tales como el IEEE 802.15.4, sufrían en su aplicación a entornos centrados en el cuerpo humano, el Institute of Electrical and Electronics Engineers (IEEE) publicó en febrero 
del año 2012 el estándar IEEE 802.15.6. Como es habitual en los estándares IEEE 802, en este únicamente se definen las características de los niveles de acceso al medio (MAC, Media Access Control) y físico (PHY) requeridos para la interconexión entre dispositivos de una red de área corporal inalámbrica. A nivel físico, el estándar contempla tres bandas de frecuencias, cada una orientada a un tipo de escenario de aplicación específico: banda estrecha (NB, Narrow Band), Ultra Wideband (UWB) y comunicaciones a través del cuerpo (HBC, Human Body Communications). De entre las diferentes bandas de frecuencias anteriores, UWB está ganando cada vez más interés entre la comunidad científica por características como las siguientes:

- Baja potencia de emisión y consumo.

- Inmunidad frente a interferencias por multicamino.

- Alta tasa de transferencia.

- Alto nivel de seguridad.

- Alta resolución temporal (y espacial).

- Hardware sencillo.

Debido a que los distintos dispositivos que conforman una WBAN suelen estar alimentados mediante baterías, la baja potencia de emisión es clave para alargar el tiempo entre reposición o carga. Esto es especialmente importante en el caso de dispositivos implantados donde la sustitución de las baterías se debe realizar mediante procedimientos quirúrgicos. Además, debido a que los dispositivos se van a encontrar dentro o en la inmediata proximidad del cuerpo, la baja potencia de emisión permite tener unos niveles bajos de radiación, cuantificado por medio de la tasa de absorción específica (SAR, Specific Absortion Rate), lo cual hace a la tecnología UWB una candidata especialmente adecuada en aplicaciones médicas (eHealth). Unido a lo anterior, la potencia de la señal transmitida se distribuye a lo largo del ancho de banda disponible, por lo que el nivel de la señal se encuentra cercano al nivel de ruido de otras tecnologías de banda estrecha, proporcionando además, un alto nivel de seguridad en las comunicaciones. En general la banda de UWB permite realizar un balance de compromiso entre alta tasa de transferencia a corta distancia y baja tasa de transferencia a larga distancia.

A pesar de las ventajas inherentes de la tecnología UWB en su aplicación a las WBAN, la dependencia con la frecuencia de las características dieléctricas de los tejidos biológicos hace que el modelado de la propagación sea en general complejo. Dependiendo de la posición relativa entre nodos de una WBAN, el canal radio presentará un comportamiento 
que debe ser modelado apropiadamente con el fin de diseñar sistemas de comunicaciones que funcionen de manera óptima en esta banda.

De entre los distintos tipos de canales en los cuales el empleo de UWB aporta un valor diferenciador se puede destacar el canal off-body entre un dispositivo sobre la superficie del cuerpo y otro en el entorno de este y, en los últimos años, el canal in-body entre un dispositivo en el interior del cuerpo y otro colocado sobre la superficie o a una cierta distancia del mismo. Debido al reducido número de estudios asociados al modelado de los dos tipos de canales anteriores, se ha planteado la presente tesis con el propósito de abordar los siguientes objetivos:

1) Revisión del estado del arte en el campo de la propagación en redes de área corporal inalámbricas para la banda de UWB de 3.1-10.6 GHz.

2) Desarrollo de procedimientos de medidas para la caracterización en banda ancha del canal radio off-body e in-body en UWB.

3) Caracterización de la propagación off-body en entornos indoor y bajo condiciones de visión directa (LOS, Line of Sight) entre una estación base y dispositivos colocados en diferentes partes del cuerpo de un sujeto considerando el canal estático para UWB.

4) Caracterización de los efectos derivados de la variación en el tiempo de la posición relativa entre dos dispositivos de una WBAN, al menos uno de ellos ubicado en el interior del cuerpo, debido a la respiración.

5) Verificación de los modelos con las medidas experimentales.

\subsection{Estructura de la tesis}

En este apartado se va a describir cada uno de los capítulos en los cuales se estructura la presente tesis:

En el Capítulo 2 se presenta un marco introductorio a las redes de área corporal inalámbricas, considerando sus elementos constitutivos, bandas de frecuencia, estandarización y modelos de canal. Además, se introduce los fundamentos de la tecnología UWB y su aplicación a las redes WBAN.

En el Capítulo 3 se presentan los resultados correspondientes a la caracterización del canal de propagación WBAN off-body para la banda de UWB, entre un punto de acceso transmisor colocado a una distancia máxima de tres metros respecto a un dispositivo receptor colocado sobre la superficie del cuerpo de un sujeto. En primer lugar, se evalúa el efecto de 
las características del entorno de medidas en el canal de propagación, cuantificado en términos de las pérdidas de propagación y la dispersión de retardo del canal radio. A continuación, se presenta el estudio de la influencia de la posición de colocación de una antena receptora sobre el cuerpo de un conjunto de sujetos en el canal de propagación offbody, considerando condiciones de visión directa y sujetos de pie. Por último, se presenta un estudio sobre la variación en las características del canal off-body UWB en función de la postura adoptada por un sujeto: de pie y tumbado. Adicionalmente para cada postura, se realiza un modelado de la respuesta al impulso del canal empleando el modelo CM4 propuesto por el estándar IEEE 802.15.6 para UWB, así como la comparación de los valores obtenidos con estudios previos.

En el Capítulo 4 se presentan los resultados de la caracterización del canal de propagación in-body, considerando el movimiento relativo entre dos dispositivos de una WBAN causado por efecto de la respiración. El modelado de las condiciones de propagación en el interior del cuerpo se realiza por medio de un phantom líquido diseñado para emular la respuesta dieléctrica de los tejidos humanos con alto contenido en agua (como los músculos) en la banda de 3.1-10.6 GHz. Se consideran tres tipos de canales distintos atendiendo a la colocación de los dispositivos respecto al cuerpo: canal in-body a on-body considerando un dispositivo en el interior del cuerpo y otro en la superficie del mismo, canal in-body a offbody entre un dispositivo colocado en el interior del cuerpo y otro exterior a una cierta distancia, y por último, canal in-body a in-body considerando dos dispositivos en el interior del cuerpo. De esta forma se presenta la caracterización tanto en frecuencia, en términos del modelado de la forma del espectro Doppler y la anchura del mismo, como en el tiempo por medio del modelado de la autocorrelación y el tiempo de coherencia.

En el Capítulo 5 se presentan las principales conclusiones extraídas a partir de los estudios realizados en la presente tesis sobre la caracterización de la propagación en redes WBAN para la banda de UWB considerando condiciones de canal estático y dinámico. Así mismo, se listan algunas de las posibles líneas de investigación planteadas en el ámbito del modelado de la propagación en redes de área corporal inalámbricas.

\subsection{Publicaciones científicas}

La presente tesis ha dado como resultado 2 publicaciones internacionales (IJ) en una revista indexada en el Journal Citations Report (JCR), 2 congresos internacional (IC) y 1 congreso nacional (NC). 
Los resultados derivados de los estudios desarrollados en el capítulo 3 han sido publicados en:

IJ1 Ruben-Gregorio Garcia-Serna, Concepcion Garcia-Pardo and Jose-Maria MolinaGarcia-Pardo, "Effect of the Receiver Attachment Position on Ultra Wideband OffBody Channels", IEEE Antennas and Wireless Propagation Letters, vol. 14, pp. 1101-1104. January 2015. Digital Object Identifier: 10.1109/LAWP.2015.2394737.

IC1 Ruben-Gregorio Garcia-Serna, Concepcion Garcia-Pardo, Jose-Maria MolinaGarcia-Pardo, "Influence of the Posture in Body Surface to External UWB Body Area Networks Channels", IEEE Antennas and Propagation Symposium. Orlando, Estados Unidos de América. 7-13 July 2013.

IC2 Ruben-Gregorio Garcia-Serna, Jose-Maria Molina- Garcia-Pardo, Leandro Juan Llácer, Concepcion Garcia-Pardo and Narcis Cardona, "Initial Phantom Measurements of the Doppler Effect During Respiration in BAN", IEEE Antennas and Propagation Symposium. Fajardo, Puerto Rico. 26 June - 1 July 2016.

Por otro lado, los resultados correspondientes a los estudios desarrollados en el capítulo 4 han sido publicados en:

NC1 Ruben-Gregorio Garcia-Serna, Jose-Maria Molina- Garcia-Pardo, Leandro Juan Llácer, Concepcion Garcia-Pardo and Narcis Cardona, "Caracterización del Efecto Doppler Durante la Respiración en Redes de Área Corporal", XXXI Simposium nacional de la unión científica internacional de radio URSI, Madrid, Spain. 5-7 September 2016.

IJ2 Ruben-Gregorio Garcia-Serna, Member, IEEE, Jose-Maria Molina-Garcia-Pardo, Concepcion Garcia-Pardo, Leandro Juan-Llacer, Senior Member, IEEE and Narcis Cardona, Senior Member, IEEE, "Dynamic Characterization of In-Body UWB Channels During Breathing", IEEE Antennas and Wireless Propagation Letters, 2017 (Enviado). 


\section{Capítulo 2}

\section{Redes de Área Corporal}

En este capítulo se presenta una introducción a las redes de área corporal inalámbricas o WBAN, con el fin de establecer un marco de referencia para el resto de capítulos de la presente tesis. Para ello, en primer lugar se describen los elementos constitutivos y los posibles escenarios de aplicación de este tipo de redes. Tras esto se detallan las diferentes tecnologías de transmisión inalámbrica propuestas para su aplicación en enlaces entre dispositivos de una WBAN.

A continuación, se presenta el estado actual de la estandarización en el área materializado en el estándar IEEE 802.15.6, haciendo especial mención al empleo de la banda UWB, debido a las características inherentes que hacen de esta una candidata ideal para la implementación del nivel físico. Así mismo, se presenta una introducción a esta tecnología.

Por último, se detallan los diferentes tipos de canales de propagación entre dispositivos de una WBAN y las características propias de la propagación en entornos centrados en el cuerpo y, en particular, la propagación en la banda de UWB. Además, se presentan los modelos contemplados en el estándar IEEE 802.15.6 destinados a la caracterización de la respuesta al impulso en canales radio entre dispositivos WBAN para UWB. 


\subsection{Introducción}

A la estructura formada por un conjunto de dispositivos, capaces de medir un determinado aspecto del entorno físico que les rodea, desplegados en diferentes ubicaciones del cuerpo humano (aunque puede extrapolarse a animales), organizados y con capacidad de establecer enlaces inalámbricos entre ellos se le denomina red de área corporal inalámbrica.

En una red WBAN se pueden distinguir dos tipos de dispositivos: dispositivos sensores, diseñados para medir un cierto parámetro físico, ya sea del propio cuerpo o del entorno que le rodea, y nodos concentradores (hub) a los cuales se conectan los primeros para transmitir los datos recogidos. Los nodos sensores poseen, por lo general, poca capacidad de proceso y batería en comparación con los nodos concentradores. Adicionalmente, los nodos sensores pueden tener capacidad de actuación (nodos actuadores), permitiendo llevar a cabo ciertas acciones, como puede ser en aplicaciones médicas la liberación de un medicamento.

Dependiendo del ámbito de interconexión entre dispositivos de una WBAN, se pueden identificar los siguientes tres niveles [Maduranga et al., 2014], [Movassaghi et al., 2014], representados en la Figura 2.1:

- Nivel 1 (intra-ban): interconexión entre dispositivos desplegados en el cuerpo y un elemento central que actúe de servidor personal (PS, Personal Server).

- Nivel 2 (inter-ban): interconexión entre un PS y un punto de acceso (AP, Access Point) por medio de una tecnología inalámbrica de corto alcance/red local.

- Nivel 3 (beyond-ban): interconexión entre un AP y un sistema remoto haciendo uso de una tecnología de transmisión de larga distancia.

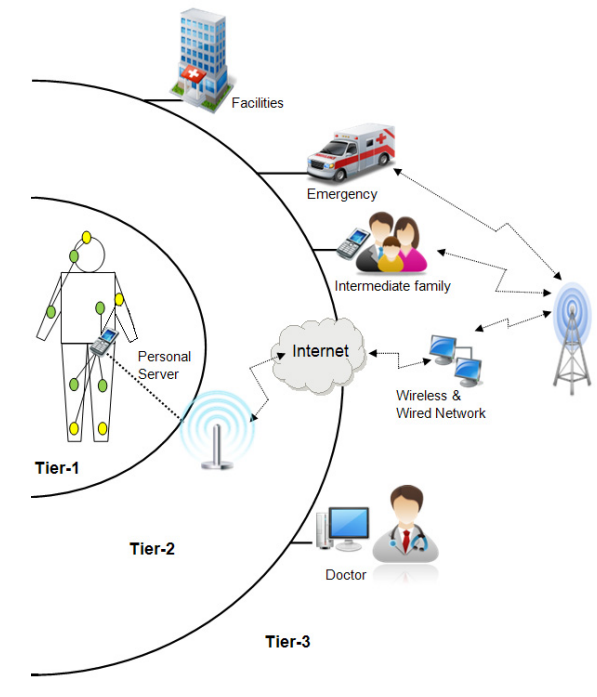

Figura 2.1: Niveles de interconexión en WBAN [Movassaghi et al., 2014]. 
Los datos obtenidos por los dispositivos desplegados en el cuerpo son, en un primer nivel, transmitidos a un dispositivo que actúe de servidor personal con mayor capacidad de procesado y almacenamiento. Este dispositivo puede fusionar los datos recogidos por los diferentes sensores y tener cierta lógica de procesado. Los datos almacenados son transmitidos a un segundo nivel por medio de un punto de acceso que puede poseer una doble funcionalidad: por un lado interconectar distintas WBAN y por otro conectar, por medio de una tecnología de red inalámbrica de larga distancia, con un sistema remoto en el tercer nivel donde la información es almacenada y procesada según la aplicación específica. Aunque esta estructuración en niveles permite separar los requerimientos y tecnologías de interconexión, es dependiente de la aplicación en concreto ya que, por ejemplo, el servidor personal del primer nivel puede sustituirse por un dispositivo, tal como teléfono móvil 3G/4G, con capacidad de transmitir los datos directamente a los sistemas del tercer nivel.

La posibilidad de monitorizar diferentes aspectos del cuerpo humano, desde las constantes vitales hasta el movimiento y el entorno que le rodea, permite plantear multitud de aplicaciones para las redes WBAN. De manera general, se puede establecer la siguiente clasificación de tipos de aplicaciones [Wang y Wang, 2013], [Hall y Hao, 2012]:

- Aplicaciones médicas: diagnóstico médico remoto, monitorización continua de constantes vitales, rehabilitación, etc.

- Asistencia a personas con minusvalías: asistencia a personas invidentes, asistencia al habla, miembros artificiales, sistemas de prevención de caídas para personas mayores, etc.

- Electrónica de consumo e identificación personal: auriculares inalámbricos, difusión de audio/video a grupos, identificación personal, pago automático, etc.

- Navegación y servicios basados en la localización: turismo, sistemas de transporte inteligente (ITS, Intelligent Transport Systems), etc.

Aunque cada tipo de aplicación anterior presenta unos requerimientos específicos, el éxito en el empleo de una red WBAN pasa por considerar al menos los siguientes requisitos básicos [Negra et al., 2016]:

- Fiabilidad.

- Latencia.

- Seguridad de la información.

- Consumo energético. 
Debido a la naturaleza de los datos recogidos por los distintos dispositivos desplegados en el cuerpo, ya sea en el interior o en el exterior del mismo, la fiabilidad y la seguridad de los mismos es fundamental. La información recogida debe caracterizar de manera precisa la variable física objetivo y el sistema debe asegurar que los valores no son susceptibles de ser modificados/suplantados por un posible atacante durante la transferencia de los mismos hasta el sistema final. Además, usualmente los datos deben transmitirse con la mínima latencia posible, ya que de lo contrario, puede que no tengan validez o, por ejemplo en aplicaciones médicas, esto suponga graves riesgos para la salud de un paciente. Por último, debido a que los dispositivos usualmente se alimentan por baterías, las aplicaciones deben optimizar el consumo para alargar al máximo el tiempo entre reemplazo/carga de las mismas.

\subsection{Tecnologías inalámbricas en redes de área corporal}

Una de las principales ventajas que aportan las redes de área corporal inalámbricas es precisamente la libertad de movimiento que ofrecen a los usuarios al no estar limitados por la rigidez debida a una estructura de interconexión entre dispositivos cableada. Ya que los enlaces entre dispositivos que conforman la red se hace de manera inalámbrica, la selección de la tecnología de transmisión es de vital importancia a la hora de garantizar los parámetros de operación requeridos en la aplicación objetivo. Según la naturaleza de información recogida por la red, los requerimientos en cuanto a ancho de banda, seguridad, tasa de error de bit (BER, Bit Error Rate), calidad de servicio, distancia de operación, etc., serán diferentes. En la Tabla 2.1. se describen los diferentes requerimientos que presentan algunas de las aplicaciones WBAN más habituales.

\begin{tabular}{|c|c|c|c|}
\hline Aplicación & Tasa de datos & Latencia & BER \\
\hline $\begin{array}{c}\text { Administración de } \\
\text { fármacos }\end{array}$ & $<16 \mathrm{kbps}$ & $<250 \mathrm{~ms}$ & $<10^{-10}$ \\
\hline $\begin{array}{c}\text { Estimulación cerebral } \\
\text { profunda }\end{array}$ & $<320 \mathrm{kbps}$ & $<250 \mathrm{~ms}$ & $<10^{-10}$ \\
\hline Cápsula endoscópica & $1 \mathrm{Mbps}$ & - & $<10^{-10}$ \\
\hline ECG & $\begin{array}{c}192 \mathrm{kbps} \\
(6 \mathrm{Kbps}, 32 \mathrm{canales})\end{array}$ & $<250 \mathrm{~ms}$ & $<10^{-10}$ \\
\hline EEG & $\begin{array}{c}86.4 \mathrm{kbps} \\
(300 \mathrm{~Hz} \text { muestra, } 12-\end{array}$ & $<250 \mathrm{~ms}$ & $<10^{-10}$ \\
\hline bit ADC, $24 \mathrm{canales})$ & $\begin{array}{c}1.536 \mathrm{kbps} \\
(8 \mathrm{kHz} \text { muestra, } 16-\mathrm{bit} \\
\text { ADC, } 12 \mathrm{canales})\end{array}$ & $<250 \mathrm{~ms}$ & $<10^{-10}$ \\
\hline EMG & $<1 \mathrm{kbps}$ & $<250 \mathrm{~ms}$ & $<10^{-10}$ \\
\hline Monitor de glucosa & $1 \mathrm{Mbps}$ & $<20 \mathrm{~ms}$ & $<10^{-5}$ \\
\hline Audio & $<10 \mathrm{Mbps}$ & $<100 \mathrm{~ms}$ & $<10^{-3}$ \\
\hline Video & $50-100 \mathrm{kbps}$ & $<10 \mathrm{~ms}$ & $<10^{-3}$ \\
\hline Voz & & & \\
\hline & & & \\
\hline
\end{tabular}

Tabla 2.1: Requisitos de operación para distintas aplicaciones WBAN [Cordeiro, 2007]. 
Con el propósito de implementar los enlaces inalámbricos entre dispositivos desplegados en una WBAN, se han propuesto en los últimos años distintas tecnologías candidatas, un resumen de las cuales se presenta en la Tabla 2.2. Debido a que el ámbito de aplicación de las redes de área corporal es muy diverso, la elección de una tecnología u otra vendrá dado por el cumplimiento de alguna de las siguientes premisas básicas [Maduranga et al., 2014]:

- Tasa de datos escalable.

- Bajo consumo.

- Bajo factor de forma.

- Potencia de transmisión controlable.

- Tráfico con prioridad.

- Seguridad en las transmisiones.

- Coexistencia con otras tecnologías inalámbricas.

- Acceso multiusuario.

\begin{tabular}{|c|c|c|c|c|}
\hline $\begin{array}{c}\text { Tecnología } \\
\text { inalámbrica }\end{array}$ & $\begin{array}{l}\text { Distancia } \\
\text { máxima }\end{array}$ & Tasa de datos & $\begin{array}{l}\text { Número de } \\
\text { nodos }\end{array}$ & $\begin{array}{c}\text { Banda de } \\
\text { frecuencias } \\
(\mathrm{MHz}) \\
\end{array}$ \\
\hline RFID Pasivo & $0-3 \mathrm{~m}$ & $<848 \mathrm{kbps}$ & $1+$ lector & $\begin{array}{c}13.5 \\
860-960\end{array}$ \\
\hline RFID Activo & $0-100 \mathrm{~m}$ & $>10 \mathrm{Mbps}$ & $\begin{array}{c}\text { Hasta } 1000+ \\
\text { lector }\end{array}$ & 433 \\
\hline $\begin{array}{c}\text { MedRadio/ } \\
\text { Zarlink }\end{array}$ & $2 \mathrm{~m}$ & $200-800 \mathrm{kbps}$ & N/A & $\begin{array}{l}402-405 \\
433-434 \\
\end{array}$ \\
\hline MICS & $0-10 \mathrm{~m}$ & 19 ó $76 \mathrm{kbps}$ & N/A & $402-405$ \\
\hline $\begin{array}{l}\text { Wireless } \\
\text { Medical } \\
\text { Telemetry } \\
\text { System } \\
\text { (WMTS) }\end{array}$ & $30-60 \mathrm{~m}$ & $>250 \mathrm{kbps}$ & N/A & $\begin{array}{c}608-614 \\
1395-1400 \\
1429-1432\end{array}$ \\
\hline Sensium & $1-5 m$ & $50 \mathrm{kbps}$ & $8+1$ & $\begin{array}{l}868-870 \\
868-915 \\
902-928 \\
\end{array}$ \\
\hline $\begin{array}{c}\text { Bluetooth/ } \\
802.15 .1\end{array}$ & $1-10 \mathrm{~m}$ & 1-3 Mbps & $7+1$ & $2400-2500$ \\
\hline Bluetooth LE & $1-10 \mathrm{~m}$ & $1 \mathrm{Mbps}$ & $7+1$ & $2400-2500$ \\
\hline $\begin{array}{c}\text { Zigbee/ } \\
802.15 .4 \\
\end{array}$ & $10-100 \mathrm{~m}$ & $<250 \mathrm{kbps}$ & Sin límite & $2400-2500$ \\
\hline ANT & $10-30 \mathrm{~m}$ & $1 \mathrm{Mbps}$ & $\mathrm{N} / \mathrm{A}$ & $2400-2500$ \\
\hline $\begin{array}{c}\text { WLAN } \\
802.11 \mathrm{a} / \mathrm{b} / \mathrm{g} / \mathrm{n}\end{array}$ & $\begin{array}{c}35-120 \mathrm{~m} \\
(\mathrm{a} / \mathrm{b} / \mathrm{g}) \\
70-250 \mathrm{~m}(\mathrm{n})\end{array}$ & $54-150 \mathrm{Mbps}$ & 255 & $\begin{array}{l}2400-2500 \\
5725-5850\end{array}$ \\
\hline UWB & $3-10 \mathrm{~m}$ & 53-480 Mbps & $127+1$ & $3100-10600$ \\
\hline
\end{tabular}

Tabla 2.2: Comparativa de tecnologías inalámbricas para WBAN. 
De las tecnologías identificadas en la Tabla 2.2, cabe destacar las siguientes:

- Zigbee: es una tecnología basada en el estándar IEEE 802.15.4, orientada a la interconexión de dispositivos que requieran una baja tasa de transferencia a la vez que bajo consumo. A nivel físico (PHY), el estándar define varias bandas de frecuencia para la transmisión de datos soportando un canal de $20 \mathrm{kbps}$ en la banda de $868 \mathrm{MHz}, 10$ canales de $40 \mathrm{kbps}$ en la banda de $915 \mathrm{MHz}$ y 16 canal es de 256 kbps en la banda de $2.4 \mathrm{GHz}$.

- Wireless Local Area Network (WLAN): por su implantación es la tecnología de red inalámbrica más extendida. Se basa en la norma IEEE 802.11 sobre la que se han definido diferentes variantes $(\mathrm{a} / \mathrm{b} / \mathrm{g} / \mathrm{n}$, etc.) en las cuales cada vez se ha ido aumentando más la tasa de transferencia. Opera en la banda ISM principalmente en la banda de $2.4 \mathrm{GHz}$ y $5 \mathrm{GHz}$.

- Medical Implant Communication Services (MICS): es una tecnología diseñada para establecer enlaces entre implantes. Opera en la banda de 401-406 MHz sobre la que hay asignados 10 canales de $300 \mathrm{kHz}$ cada uno.

- Bluetooth: junto con la tecnología WLAN es la segunda con mayor nivel de implantación. Tanto la variante tradicional como la LE (Low Energy) operan en la banda ISM (Industrial, Scientific and Medical) de $2.4 \mathrm{GHz}$ entre $2402 \mathrm{MHz}$ y $2480 \mathrm{MHz}$ sobre 79 canales de $1 \mathrm{MHz}$.

- Ultra Wideband (UWB): es una tecnología que opera en la banda regulada inicialmente por la Federal Communications Commision (FCC) de 3.1-10.6 GHz con canales de hasta $500 \mathrm{MHz}$. Proporciona una baja potencia de emisión, bajo consumo y alta tasa de transferencia.

En su aplicación a entornos WBAN, sobre todo en aplicaciones médicas, algunas de las tecnologías anteriores presentan serios problemas. Tanto Zigbee como Bluetooth y WLAN operan en la misma banda ISM por lo tanto son susceptibles de interferirse entre ellas o ser interferidas por otras tecnologías o sistemas que operen en la misma banda. Además, ya que los dispositivos en una WBAN deben estar alimentados por medio de baterías, se requiere que el consumo sea el mínimo posible para alargar el reemplazo de las mismas lo más posible. Respecto a esto, aunque Zigbee posee unos niveles de consumo entre $17.4 \mathrm{~mA}$ y 19.7 mA, bajos comparados con los niveles de consumo presentes en Bluetooth $(<30 \mathrm{~mA}) \mathrm{o}$ WLAN (82 mA), siguen siendo altos. Además, las tres tecnologías tienen problemas de escalado, lo cual representa una importante limitación en configuraciones en las cuales se tengan cientos de nodos desplegados. 
Muchas aplicaciones WBAN requieren de un gran ancho de banda, como por ejemplo la transferencia de video en tiempo real desde una cápsula endoscópica, por lo que la mayoría de las tecnologías anteriores, a excepción de UWB, al no ser capaces de proporcionar tasas de transferencia por encima de $1 \mathrm{Mbps}$, no constituyen soluciones viables. Con el fin de poder hacer frente a los requerimientos en cuanto a ancho de banda, calidad de servicio, seguridad y bajo consumo de las aplicaciones WBAN, la tecnología UWB se presenta como una candidata ideal. Uno de los aspectos más importantes de esta tecnología es el enorme ancho de banda disponible, y por lo tanto la tasa de transferencia superior a la alcanzable por cualquier otra de las tecnologías de banda estrecha consideradas. Además, debido a que la potencia transmitida se distribuye a lo ancho del ancho de banda disponible, el nivel de interferencia sobre otros sistemas operando en bandas vecinas es casi inexistente ya que cae por bajo del nivel de ruido de estas. Esto además tiene el beneficio adicional de la seguridad, ya que presenta un nivel muy bajo de detección.

\subsection{Estandarización en WBAN. El estándar IEEE 802.15.6}

Con el fin de hacer frente a las deficiencias que presentan los estándares del IEEE previamente diseñados para el desarrollo de redes de área personal (PAN), tales como el IEEE 802.15.3 y el IEEE 802.15.4, debido a la interacción de los dispositivos con el cuerpo humano, en noviembre de 2007 se constituyó el grupo de trabajo (TG, Task Group) TG6 con el fin de desarrollar un estándar de comunicaciones entre dispositivos desplegados en el interior, sobre la superficie o a una cierta distancia del cuerpo asegurando un nivel de fiabilidad, calidad de servicio (QoS, Quality of Service), baja potencia de emisión, alta tasa de transferencia (hasta $10 \mathrm{Mbps}$ ), alto nivel de seguridad y sin interferencia orientado al desarrollo de las redes WBAN, no únicamente limitado a seres humanos. El estándar IEEE 802.15.6 se presentó en febrero del 2012 y en él se especifican tanto las características del nivel físico (PHY) como las del nivel de enlace (MAC) a cumplir por los dispositivos de una WBAN [IEEE 802.15.6, 2012]. Los requerimientos funcionales, los cuales el estándar pretende cubrir son los listados en la Tabla 2.3. [TG6 TRD, 2008]. 


\begin{tabular}{|c|c|}
\hline Topología & Estrella (1 salto), árbol (2 saltos) \\
\hline Tiempo de configuración & Inserción/eliminación $<3 \mathrm{~s}$ \\
\hline Número de dispositivos & Típico 6, hasta 256 \\
\hline Tasa de datos & $10 \mathrm{Kbps}-10 \mathrm{Mbps}$ \\
\hline Distancia & $\approx 3 \mathrm{~m}$ \\
\hline PER & $<10 \%$ para una carga de 256 bytes \\
\hline Latencia & $\begin{array}{c}<125 \text { ms (aplicaciones médica), }<250 \\
\text { (aplicaciones no médicas) }\end{array}$ \\
\hline Jitter & $<50 \mathrm{~ms}$ \\
\hline Fiabilidad & $\begin{array}{c}<1 \text { s para alarmas, }<10 \text { ms para aplicaciones } \\
\text { con feedback }\end{array}$ \\
\hline Consumo & $\begin{array}{l}>1 \text { año (1\% LDC }+500 \mathrm{mAh} \text { batería }) ;>9 \mathrm{~h} \\
\text { (always ON }+50 \mathrm{mAh} \text { batería) }\end{array}$ \\
\hline Densidad & Hasta 10 BAN en un volumen de 6x6x6 m \\
\hline Potencia Tx & $0.1 \mathrm{~mW}$ \\
\hline $\begin{array}{l}\text { Índice de Absorción } \\
\text { Especifico (SAR) }\end{array}$ & $<10 \mathrm{dbm}$ (en la superficie) \\
\hline
\end{tabular}

Tabla 2.3: Requisitos funcionales del estándar IEEE 802.15.6.

En el estándar se definen dos tipos de dispositivos presentes en una WBAN: nodos y hubs (nodos concentradores/coordinadores). Los nodos son dispositivos sensores heterogéneos con recursos limitados en cuanto a capacidad de procesamiento, batería, memoria y transmisión de datos. Estos se pueden, a su vez, clasificar de la siguiente forma:

- Nodos implantados: son aquellos nodos colocados en el interior del cuerpo humano, justo debajo de la superficie o a mayor profundidad (marcapasos, bombas de insulina, etc.).

- Nodos en la superficie del cuerpo: son aquellos nodos colocados en la superficie del cuerpo o a una separación máxima de $2 \mathrm{~cm}$.

- Nodos externos: son aquellos nodos que no tienen contacto directo con la superficie del cuerpo humano. Se pueden considerar como nodos externos todos aquellos colocados entre unos centímetros y $5 \mathrm{~m}$ de separación respecto al cuerpo.

Por otro lado, los hubs o coordinadores son dispositivos con mayores recursos destinados a servir de concentradores para los datos enviados por el resto de nodos de la WBAN y enlace, por medio de una tecnología radio de larga distancia, con otros elementos remotos del sistema. Además, estos dispositivos son los responsables de coordinar el acceso al medio y la gestión de potencia de los nodos de la red. En toda WBAN únicamente puede haber un nodo hub mientras que pueden existir hasta 256 nodos.

En el estándar IEEE 802.15.6 se definen dos topologías de interconexión entre los diferentes dispositivos de la red: estrella y estrella extendida (o en árbol restringido). Estas topologías implican conexión directa (one-hop) de los nodos con el hub para el caso de la 
conexión en estrella, o bien conexión a través de otro nodo intermedio (two-hop) para el caso de la topología en estrella ampliada o árbol. En la Figura 2.2. se muestran las dos posibles topologías de red contempladas.

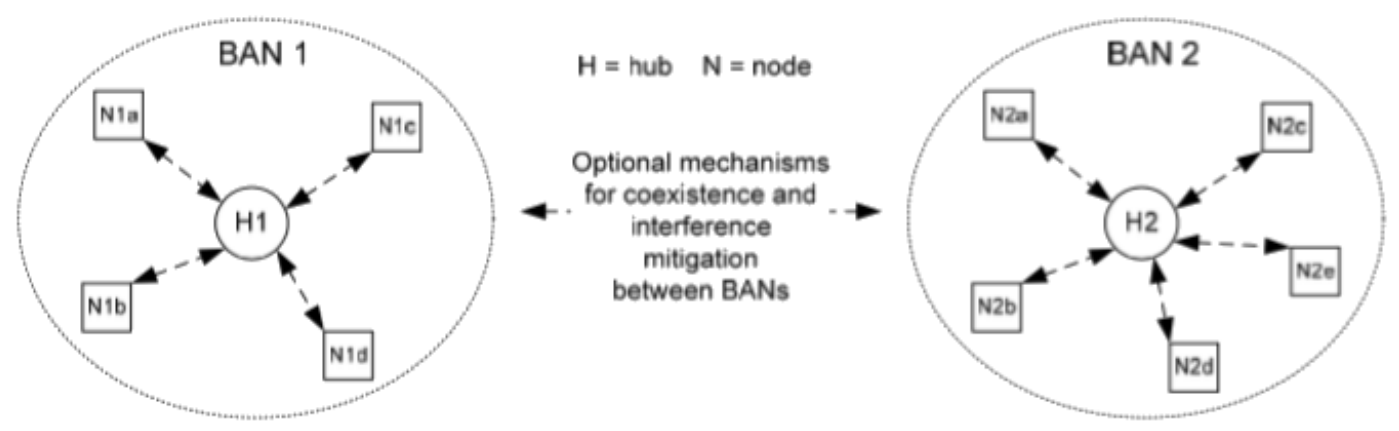

(a)

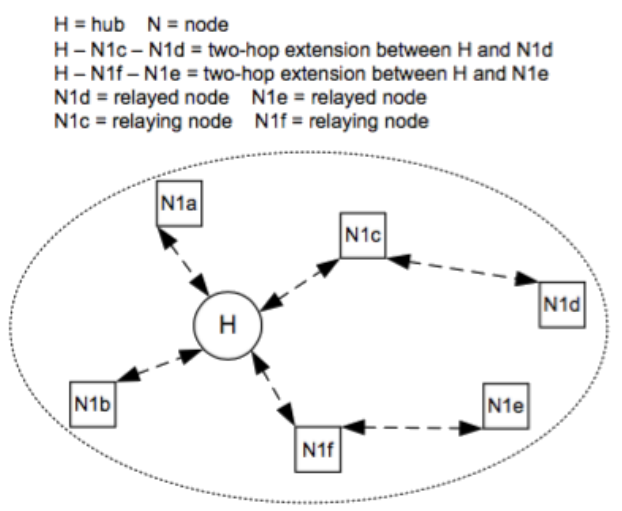

(b)

Figura 2.2: Topologías de red contempladas en el estándar IEEE 802.15.6. (a) Topología en estrella, (b) topología en estrella extendida [IEEE 802.15.6, 2012].

Respecto a la regulación sobre el nivel máximo de potencia emitida la norma únicamente remite a las regulaciones propias de cada país. Por ejemplo, para la banda Medical Implants Communication Services (MICS) de 402-405 MHz, en el caso de Europa, la ETSI establece un nivel de potencia máximo de emisión de $25 \mu \mathrm{W}$ ERP.

\subsubsection{EI nivel de acceso al medio (MAC)}

Este nivel es el encargado de arbitrar el acceso al canal. El coordinador (hub) establece una base de referencia de tiempos, la cual se estructura como una concatenación de periodos de baliza (beacon periods) o supertramas de igual longitud, a su vez cada una de estas, compuesta por intervalos de asignación de recursos (numerados de 0 a 255).

Debido a las diferentes aplicaciones y flujos de datos a los que el estándar debe dar soporte, se definen tres posibles modos de acceso seleccionables por el hub de una WBAN: 
- Con baliza y con delimitadores de supertrama.

- Sin baliza y con delimitadores de supertrama.

- Sin baliza y sin delimitadores de supertrama.

En el primer modo, el coordinador envía (salvo que por regulación esté prohibido, como es en la banda MICS) paquetes baliza (beacons) en cada periodo de baliza para indicar el inicio de cada supertrama. La supertrama, como se puede ver en la Figura 2.3 (a), se divide a su vez en fase de acceso exclusivo (EAP, Exclusive Acces Phase), fase de acceso aleatorio 1 (RAP1, Random Access Phase 1), EAP2, RAP2, fase de acceso gestionado (MAP, Managed Access Phase) y fase de acceso por contienda (CAP, Contention Access Phase). Las fases EAP1 y EAP2 se emplean para tráfico de alta prioridad, mientras que RAP1, RAP2 y CAP se emplean únicamente para tráfico normal y el acceso a los recursos se realiza por contienda. Por último, la fase MAP se emplea básicamente para tráfico basado en peticiones.

En el segundo modo, el hub únicamente tiene una fase MAP en toda supertrama, tal y como se muestra en la Figura 2.3 (b).

Por último, en el tercer modo tal y como se muestra en la Figura 2.3 (c), un hub debe proporcionar intervalos de asignación no planificados. Tras determinar que el siguiente intercambio de tramas va a tener lugar en este modo, un nodo debe tratar cualquier intervalo de tiempo como EAP1 o RAP1 y llevar a cabo el acceso al medio por medio de un procedimiento de contienda.

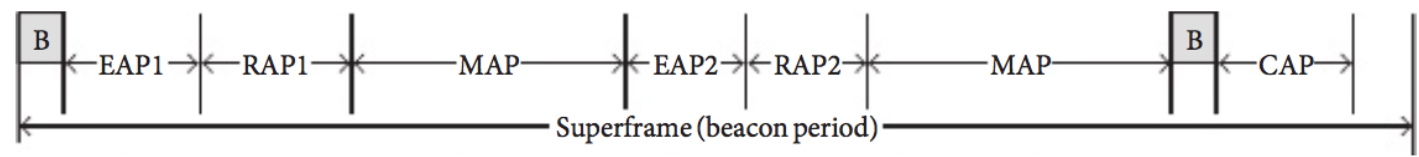

(a)

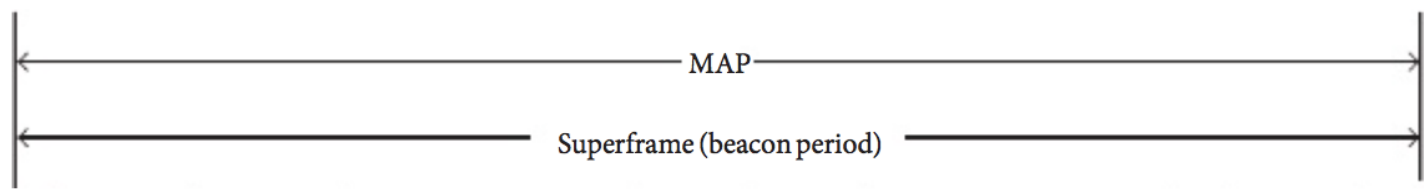

(b)

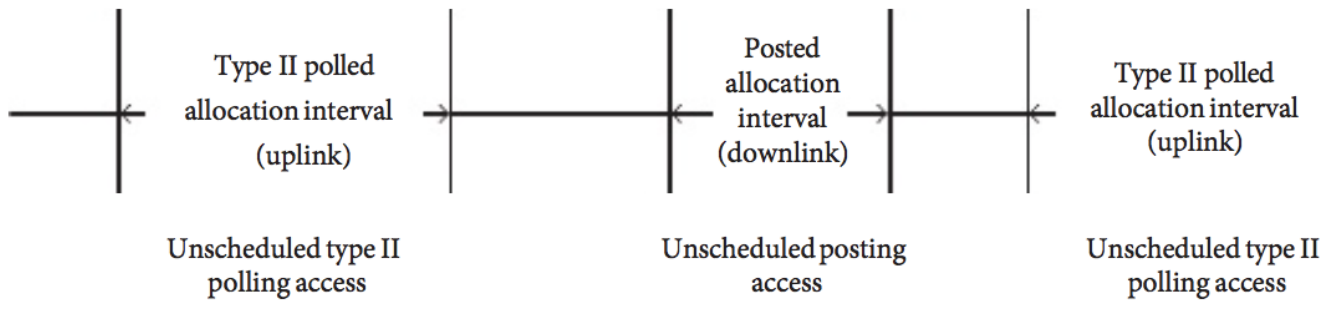

(c)

Figura 2.3: Modos de acceso en IEEE 802.15.6. (a) Con baliza y delimitadores de trama, (b) sin baliza y delimitadores de trama, (c) sin baliza ni delimitadores de trama [Ullah et al., 2013]. 
El mecanismo de acceso al medio empleado en cada una de las fases descritas anteriormente puede dividirse en las siguientes tres categorías:

- Acceso aleatorio (empleando slotted Aloha o CSMA/CD dependiendo de la implementación de nivel físico utilizada).

- Acceso improvisado sin planificar.

- Acceso planificado y sondeo planificado.

\subsubsection{El nivel físico (PHY)}

Entre las responsabilidades de este nivel están la activación y desactivación de los transceptores radio, la detección de actividad en el canal actual (CSA, Clear Channel Assessment) y el envío y recepción de datos. En el estándar IEEE 802.15.6 se proponen tres posibles implementaciones para el nivel físico:

- Narrow Band (NB): comunicaciones en banda estrecha.

- Human Body Communicacions (HBC): comunicaciones empleando el cuerpo como medio canal.

- Ultra Wideband (UWB): comunicaciones en banda ancha.

De las tres propuestas anteriores, únicamente se consideran de obligado cumplimiento UWB y HBC, quedando NB como opcional.

\subsubsection{Narrow Band (NB)}

La implementación del nivel físico por medio de tecnologías de banda estrecha está orientado en el estándar a aplicaciones principalmente médicas, en las cuales se establezcan enlaces bidireccionales fiables de corto alcance entre dispositivos. En el estándar se proponen las diferentes bandas de frecuencias listadas en la Tabla 2.4, de forma que todo dispositivo que implemente esté nivel físico debe ser capaz de transmitir y recibir datos en alguna de ellas [IEEE 802.15.6, 2012]. 


\begin{tabular}{|c|c|c|c|c|}
\hline Frecuencias & Descripción & $\begin{array}{l}\text { Ancho de } \\
\text { Banda }\end{array}$ & Modulaciones & $\begin{array}{c}\text { Tasa } \\
\text { Transferencia }\end{array}$ \\
\hline $402-405 \mathrm{MHz}$ & MICS (Global) & $300 \mathrm{kHz}$ & $\begin{array}{c}\pi / 2 \text {-DBPSK } \\
\pi / 4 \text {-DQPSK } \\
\pi / 8 \text {-D8PSK }\end{array}$ & $57.5-455.4 \mathrm{kbps}$ \\
\hline $420-450 \mathrm{MHz}$ & WMTS (Japón) & $300 \mathrm{kHz}$ & GMSK & $57.5-187.5 \mathrm{kbps}$ \\
\hline $863-870 \mathrm{MHz}$ & WMTS (Europa) & $400 \mathrm{kHz}$ & $\begin{array}{c}\pi / 2 \text {-DBPSK } \\
\pi / 4 \text {-DQPSK } \\
\pi / 8 \text {-D8PSK }\end{array}$ & 76.6-607.1 kbps \\
\hline $902-928 \mathrm{MHz}$ & $\begin{array}{c}\text { ISM (Nueva } \\
\text { Zelanda, } \\
\text { Australia, } \\
\text { Estados Unidos) } \\
\end{array}$ & $500 \mathrm{kHz}$ & $\begin{array}{c}\pi / 2 \text {-DBPSK } \\
\pi / 4 \text {-DQPSK } \\
\pi / 8 \text {-D8PSK }\end{array}$ & 76.6-607.1 kbps \\
\hline $950-956 \mathrm{MHz}$ & ISM (Japón) & $400 \mathrm{kHz}$ & $\begin{array}{c}\pi / 2 \text {-DBPSK } \\
\pi / 4 \text {-DQPSK } \\
\pi / 8 \text {-D8PSK }\end{array}$ & 76.6-607.1 kbps \\
\hline $2.36-2.40 \mathrm{GHz}$ & $\begin{array}{l}\text { MBAN (Estados } \\
\text { Unidos) }\end{array}$ & $1 \mathrm{MHz}$ & $\begin{array}{l}\pi / 2 \text {-DBPSK } \\
\pi / 4 \text {-DQPSK }\end{array}$ & 91.9-971.4 kbps \\
\hline $\begin{array}{c}\text { Banda de } 2.4 \\
\text { GHz }\end{array}$ & ISM (Global) & $1 \mathrm{MHz}$ & $\begin{array}{l}\pi / 2 \text {-DBPSK } \\
\pi / 4 \text {-DQPSK }\end{array}$ & 91.9-971.4 kbps \\
\hline
\end{tabular}

Tabla 2.4: Bandas de frecuencias asignadas en el estándar IEEE 802.15.6 para PHY NB.

En la Tabla 2.4. se puede ver que ciertas frecuencias poseen regulaciones regionales, por lo cual, únicamente son posibles en ciertos países, como por ejemplo la banda Wireless Medical Telemetry System (WMTS), la Medical Body Area Networks (MBAN) o la Industrial, Scientific and Medical (ISM). A excepción de la banda MICS, la cual es la propuesta en el estándar para la realización de enlaces cuando al menos uno de los extremos de la comunicación es un nodo implantado, el resto pueden ser empleadas en cualquier tipo de aplicación WBAN.

Los datos proporcionados por las capas superiores deben acomodarse en una estructura de trama de nivel físico denominada Physical Layer Protocol Data Unit (PPDU). Esta se compone de preámbulo y cabecera del protocolo de convergencia de nivel físico (PLCP, Physical Layer Convergence Protocol), los cuales se emplean en recepción para sincronización temporal y demodulación de los datos, y los datos del nivel físico (PSDU, Physical-layer Service Data Unit), los cuales se conforman concatenando la cabecera y los datos del nivel MAC, además de una secuencia de comprobación de trama (FCS, Frame Check Sequence). En la Figura 2.4 se muestra la estructura de PPDU descrita.

\begin{tabular}{|c|c|c|}
\hline $\begin{array}{c}\text { Preámbulo } \\
\text { PLCP }\end{array}$ & $\begin{array}{c}\text { Cabecera } \\
\text { PLCP }\end{array}$ & PSDU \\
\hline
\end{tabular}

Figura 2.4: Estructura de PPDU para PHY NB [IEEE 802.15.6, 2012]. 


\subsubsection{Human Body Communications (HBC)}

A diferencia de UWB y NB, HBC plantea la transferencia de datos empleando como canal de propagación el propio cuerpo. Para ello, en lugar de emplear antenas se emplean electrodos en contacto directo con la piel. La banda de frecuencias contemplada en el estándar para esta implementación de este nivel físico es la centrada en $21 \mathrm{MHz}$, con $5.25 \mathrm{MHz}$ de ancho de banda. La tasa máxima de transferencia que se puede alcanzar con HBC es de $1.3125 \mathrm{Mbps}$.

La trama de nivel físico PPDU en HBC está formada por preámbulo de PLCP, delimitador de inicio de trama (SFD, Start Frame Delimiter), cabecera de PLCP y el campo de datos de nivel físico o PSDU. Adicionalmente, el campo SFD puede reemplazarse por un campo indicador de tasa de transferencia (RI, Rate Indicator). En la Figura 2.5 se muestra la estructura descrita.

\begin{tabular}{|c|c|c|c|}
\hline $\begin{array}{c}\text { Preámbulo } \\
\text { PLCP }\end{array}$ & SFD/RI & $\begin{array}{c}\text { Cabecera } \\
\text { PLCP }\end{array}$ & PSDU \\
\hline
\end{tabular}

Figura 2.5: Estructura de PPDU para PHY HBC [IEEE 802.15.6, 2012].

\subsubsection{Ultra Wideband (UWB)}

De entre las tres posibles implementaciones contempladas por el estándar IEEE 802.15.6 para el nivel físico, UWB es el especificado para proporcionar un amplio espectro de oportunidades de implementación aportando alto rendimiento, robustez, baja complejidad y muy reducida potencia de emisión, lo cual, además de ser segura en términos de nivel de radiación, proporciona un nivel muy bajo de interferencias con otros sistemas [IEEE 802.15.6, 2012].

Dentro de la banda de UWB, el estándar define dos tipos de tecnologías de transmisión:

- IR-UWB.

- FM-UWB.

La tecnología por impulsos radio o IR-UWB es la más simple de las dos y es de obligada implementación tanto por parte de los nodos como de los hubs. Permite alcanzar tasas de transferencia entre los 0.497 Mbps hasta los 15.6 Mbps. Por otro lado, FM-UWB implica la modulación en frecuencia de las señales UWB y es de implementación opcional por parte de nodos y hubs. La máxima tasa de datos alcanzable en este caso es de $250 \mathrm{Kbps}$. 
La tecnología IR-UWB posee una serie de características diferenciadoras respecto a FM-UWB que la hacen una elección preferente a la hora de implementar aplicaciones médicas en WBAN [Maduranga et al., 2014]:

- Bajo consumo de los transmisores (mayor duración de las baterías).

- Alta tasa de datos.

- Bajo perfil de los dispositivos.

- Inmunidad frente a interferencias por multicamino.

A pesar de las ventajas anteriores, la tecnología IR-UWB presenta algunos inconvenientes a resolver en su aplicación a las WBAN:

- Alta complejidad en la arquitectura del receptor.

- Susceptibilidad frente a interferencias de otros sistemas.

El estándar establece una división de la banda de frecuencias asignada a UWB en banda baja y alta, tal y como se indica en la Tabla 2.5, y todo dispositivo debe ser capaz de operar en al menos una de ellas. A su vez, cada sub-banda se encuentra dividida en un conjunto de canales, de entre los cuales son obligatorios el 1 y el 6 para el caso de IR-UWB y el 6 para el caso de FM-UWB.

\begin{tabular}{|c|c|c|c|c|}
\hline Banda & $\begin{array}{c}\text { Número de } \\
\text { Canal }\end{array}$ & $\begin{array}{c}\text { Frecuencia } \\
\text { Central (MHz) }\end{array}$ & $\begin{array}{c}\text { Ancho de } \\
\text { Banda (MHz) }\end{array}$ & Obligatoriedad \\
\hline \multirow{3}{*}{ Baja } & 0 & 3494.4 & 499.2 & Opcional \\
\cline { 2 - 5 } & 1 & 3993.6 & 499.2 & Obligatorio \\
\cline { 2 - 5 } & 2 & 4492.8 & 499.2 & Opcional \\
\hline \multirow{4}{*}{ Alta } & 3 & 6489.6 & 499.2 & Opcional \\
\cline { 2 - 5 } & 4 & 6988.8 & 499.2 & Opcional \\
\cline { 2 - 5 } & 5 & 7488.0 & 499.2 & Opcional \\
\cline { 2 - 5 } & 6 & 7987.2 & 499.2 & Obligatorio \\
\cline { 2 - 5 } & 7 & 8486.4 & 499.2 & Opcional \\
\cline { 2 - 5 } & 8 & 8985.6 & 499.2 & Opcional \\
\cline { 2 - 5 } & 9 & 9484.8 & 499.2 & Opcional \\
\hline
\end{tabular}

Tabla 2.5: Bandas de frecuencia para los canales PHY UWB.

Con el fin de establecer una clasificación respecto a los posibles tipos de aplicaciones según los requerimiento de servicio, se establecen dos posibles modos de operación:

- Por defecto (default mode).

- Alta calidad de servicio (high QoS mode). 
Mientras que el modo por defecto está destinado tanto a aplicaciones médicas como no médicas, el modo de alta QoS está destinado únicamente a aplicaciones médicas en las que la calidad de servicio sea un factor crítico. Por otro lado, en el modo por defecto, es obligatorio la implementación de IR-UWB siendo opcional FM-UWB, mientras que en el modo de alta QoS la tecnología a implementar es IR-UWB.

Previo a la transmisión física de los datos, ya sea mediante IR-UWB o de FM-UWB, los datos proporcionados por las capas superiores deben acomodarse en una estructura de trama de nivel físico o PPDU, compuesta a su vez por una cabecera de sincronización (SHR, Synchronization Header), la cabecera propia del nivel físico (PHR, Physical-layer Header) y los datos (PSDU), tal y como se muestra en la Figura 2.6.

\begin{tabular}{|c|c|c|}
\hline SHR & PHR & PSDU \\
\hline
\end{tabular}

Figura 2.6: Estructura de PPDU para PHY UWB [IEEE 802.15.6, 2012].

Para transmitir los bits de la PPDU por medio de una señal de RF se definen tres posibles esquemas de modulación digital:

- On-Off Keying (OOK).

- DBPSK/DQPSK.

- Combinación de fase CP-BFSK con modulación en frecuencia de banda ancha, FM-UWB.

En el modo por defecto la modulación OOK es de obligada implementación, mientras que las modulaciones diferenciales CBPSK/DQPSK son opcionales. Por el contrario, en el modo de alta QoS sucede lo contrario, siendo de obligada implementación las modulaciones diferenciales DBPSK/DQPSK. En la Tabla 2.6. se resume la asignación de los esquemas de modulación a cada uno de los modos de operación y tecnologías de transmisión UWB.

\begin{tabular}{|c|c|c|c|}
\hline Modo & $\begin{array}{c}\text { Tecnología } \\
\text { UWB }\end{array}$ & $\begin{array}{l}\text { Esquema de } \\
\text { modulación }\end{array}$ & $\begin{array}{c}\text { Tasa de } \\
\text { datos }\end{array}$ \\
\hline \multirow{2}{*}{ Normal } & IR-UWB & OOK & $\begin{array}{c}\geq 0.487 \\
\text { Mbps }\end{array}$ \\
\hline & FM-UWB & CP-BFSK/FM-UWB & $256 \mathrm{Kbps}$ \\
\hline $\begin{array}{l}\text { Alta } \\
\text { QoS }\end{array}$ & IR-UWB & DPSK & $\begin{array}{l}\geq 0.487 \\
\text { Mbps }\end{array}$ \\
\hline
\end{tabular}

Tabla 2.6: Modulaciones para PHY UWB según los modos de calidad de servicio. 


\subsubsection{Seguridad}

Se contemplan tres niveles de seguridad dependiendo del tipo de aplicación concreta hacia la cual esté orientada la WBAN:

- Nivel 1: $\sin$ seguridad.

- Nivel 2: autenticación sin cifrado de datos.

- Nivel 3: autenticación y cifrado de datos.

El nivel de seguridad se negocia entre nodo y hub durante el proceso de unión a la red del primero. En comunicaciones seguras unicast ambos pares de la comunicación necesitan activar una clave maestra (MK, Master Key) preestablecida o crear una nueva MK y, posteriormente generar una clave temporal (PTK, Pairwise Temporal Key) que será empleada como clave de sesión. En el caso de comunicaciones seguras multicast, los hubs necesitan distribuir al resto de nodos una clave temporal para el grupo (GTK, Group Temporal Key). La distribución segura de esta clave se hace entre el hub y cada nodo usando una PTK. La estructura completa de seguridad propuesta en el estándar IEEE 802.15.6 se muestra en la Figura 2.7.

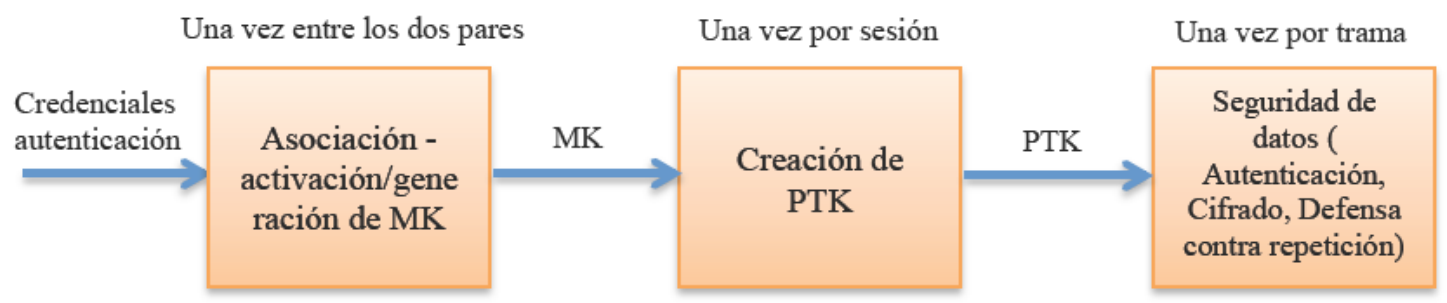

Figura 2.7: Estructura de seguridad en el estándar IEEE 802.15.6.

\subsection{Introducción a la tecnología Ultra Wideband (UWB)}

A pesar de que la tecnología de transmisión de banda ultra ancha o Ultra Wideband (UWB) ha ganado cada vez más popularidad en los últimos años, sus orígenes se remontan mucho antes. Los primeros pulsos electromagnéticos generados por parte de Heinrich Herz en 1893 o Guillermo Marconi en 1901 se pueden considerar como los precursores de los sistemas de transmisión por impulsos actuales [Di Benedetto y Giancola, 2004]. A pesar de esto, su empleo a lo largo de los años pasó a un segundo plano a favor de las transmisiones empleando señales moduladas por medio de portadoras sinusoidales. No fue sino tras la segunda Guerra Mundial y debido a los avances militares en el campo del RADAR lo que hizo que la tecnología UWB empezara a cobrar una mayor importancia. 
Se entiende por sistemas UWB aquellos para los cuales se cumple que las señales empleadas poseen un ancho de banda fraccional superior a $0.2-0.25$, o bien un ancho de banda absoluto superior a $500 \mathrm{MHz}$. Por regla general, se emplea el criterio del ancho de banda fraccional para señales inferiores a $2.5 \mathrm{GHz}$ y el criterio del ancho de banda absoluto para las superiores [Di Benedetto y Giancola, 2004]. Entendemos como ancho de banda fraccional $\left(B W_{f r a c}\right)$ al ancho de banda de la señal respecto a la frecuencia central, o lo que es lo mismo:

$$
B W_{\text {frac }}=\frac{f_{H}-f_{L}}{\left(\frac{f_{H}+f_{L}}{2}\right)}
$$

donde $f_{L}$ y $f_{H}$ son las frecuencias mínima y máximas de la señal entre las cuales la densidad espectral de potencia (PSD, Power Spectral Density) se encuentra $10 \mathrm{~dB}$ por bajo de su máximo. Adicionalmente, una importante característica de este tipo de sistemas es su baja PSD [Ghavami et al., 2005], definida como:

$$
P S D=\frac{P}{B W}
$$

donde $P_{t x}$ es la potencia total transmitida y $B W$ es el ancho de banda de la señal. Debido al tipo de señales empleadas, generalmente pulsos con anchos temporales en el rango de nano segundos, y a partir de la relación inversa entre tiempo y frecuencia, la potencia transmitida se reparte a lo largo de un enorme ancho de banda. Esto hace que este tipo de sistemas tengan una baja probabilidad de detección y baja interferencia sobre otras tecnologías de banda estrecha que operen en bandas de frecuencias adyacentes debido a que las señales UWB se encuentran bajo el nivel de ruido de estos sistemas.

Otra consecuencia del gran ancho de banda en los sistemas UWB es su efecto sobre la capacidad de los mismos. La capacidad de puede definir a partir del teorema de ShannonHartley de la siguiente forma:

$$
C=B \cdot \log \left(1+\frac{S}{N}\right)
$$

donde $C$ es la capacidad máxima del canal en bits/segundo, $B$ es el ancho de banda del canal en hercios $(\mathrm{Hz})$ y $S / N$ es la relación entre la potencia de la señal y la potencia de ruido, ambas expresadas en vatios (W). De la ecuación anterior se puede deducir que en un sistema UWB para una determinada relación señal a ruido $(S / N)$, la capacidad del canal se puede 
aumentar incrementando el ancho de banda del mismo, aunque no de manera infinita ya que al aumentar el ancho de banda también lo hace el ruido en el sistema. Esto da lugar a poder implementar sistemas de comunicaciones con una alta tasa de transferencia teniendo en cuenta las limitaciones entre distancia y relación señal a ruido.

Con el fin de establecer un marco regulatorio al uso de sistemas de banda ultra ancha, la US Federal Communications Commission (FCC) estableció en el 2002 la banda de frecuencias que podía ser usada por estos sistemas de manera libre de licencias, asegurando transmisiones sin interferencia con otros sistemas operando en bandas adyacentes por medio del empleo de una máscara que regula la potencia radiada isotrópica equivalente (EIRP, Equivalent Isotropic Radiated Power) máxima para el espectro regulado. La FCC reservó un ancho de banda de $7.5 \mathrm{GHz}$ entre $3.1 \mathrm{GHz}$ y $10.6 \mathrm{GHz}$ con una EIRP máxima inferior a $-41.3 \mathrm{dBm} / \mathrm{MHz}$.

La trasposición de la norma de la FCC a estándares en el espacio regulatorio europeo elaborados por parte del European Telecommunications Standards Institute (ETSI) a partir de los mandatos de la Comisión Europea (CE) a través del comité Electronic Communications Commitee (ECC) de la Conférence Européenne des Postes et Télécommunications (CEPT) modificó la banda total de frecuencias disponibles realizando una subdivisión en dos: de 3.1-4.8 GHz (banda baja) y de $6 \mathrm{GHz}$ a $8.5-9 \mathrm{GHz}$ (banda alta), además de introducir limitaciones adicionales en cada una de las sub-bandas y cambiar la definición de sistema UWB por medio del ancho de banda absoluto de $500 \mathrm{MHz}$ a $50 \mathrm{MHz}$ [ETSI TR 103 181-1, 2015].

Las principales limitaciones encontradas durante el proceso de regulación en el marco europeo se debieron a las mayores restricciones sobre posibles interferencias con otros sistemas previamente regulados. Debido a esto, las máscaras de emisión se variaron y adaptaron para asegurar una interferencia mínima o inexistente en las bandas compartidas. Se recomienda el uso de la banda alta con una EIRP de $-41.3 \mathrm{dBm} / \mathrm{MHz}$ hasta los $8.5 \mathrm{GHz}$ y usando mecanismos de mitigación como el Detection And Avoidance (DAA), hasta los $9 \mathrm{GHz}$. Las limitaciones sobre la banda baja son más estrictas, contemplando una EIRP de $-70 \mathrm{dBm} / \mathrm{MHz}$ en condiciones normales y $-41.3 \mathrm{dBm} / \mathrm{MHz}$ empleando técnicas de mitigación de interferencias tales como DAA o Low Duty Cycle (LDC), debido a la cantidad de sistemas que pueden ser víctima de interferencias (GPS, WiMax, etc.). Realmente se han definido diferentes máscaras de emisión adaptadas a cada escenario de aplicación (radar, aplicaciones médicas, aplicaciones vehiculares, vigilancia, sistemas indoor, etc.). Un ejemplo de las máscaras generales definidas en la regulación europea y su comparación con la máscara general contemplada en la norma de la FCC puede verse en la Figura 2.8. 


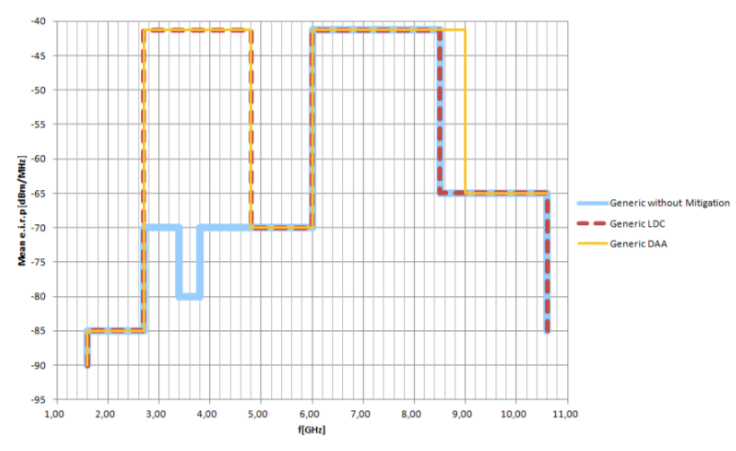

(a)

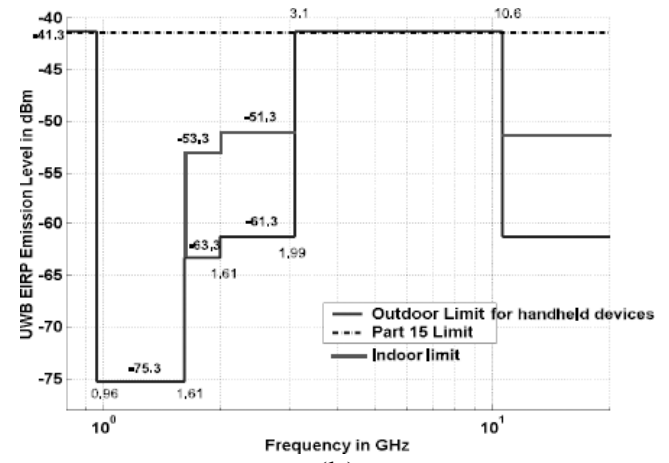

(b)

Figura 2.8: Mascaras de emisión genéricas UWB. (a) ETSI [ETSI TR 103 181-1, 2015], (b) FCC [Begaud, 2011].

Por último, tras las últimas modificaciones en los estándares europeos llevados a cabo por la ETSI, queda excluida la posibilidad de usar UWB en entornos exteriores (outdoor), restringiendo su uso a interiores. A pesad de las limitaciones impuestas en la regulación y como consecuencia, la reducción en el interés por esta tecnología en entornos generales, su uso como tecnología de enlace a corta distancia entre dispositivos en redes PAN y WBAN ha aumentado considerablemente debido a las características inherentes a la tecnología UWB, la cual la hacen especialmente atractiva en entornos médicos y aplicaciones en las cuales se requiera una alta tasa de transferencia de datos.

\subsection{El canal de propagación WBAN}

El diseño de sistemas de comunicaciones que funcionen de manera óptima en escenarios en los cuales el cuerpo humano constituya un elemento central requiere de la adecuada caracterización de las condiciones que este impone sobre la propagación de señales.

Las particulares características que ejerce el cuerpo humano, en cuanto a la propagación de señales se refiere, hacen que en general la caracterización del canal de propagación radio en una WBAN sea complicada y, por lo tanto, difícil establecer un modelo único que sea válido para todos los posibles escenarios. De manera general, el cuerpo humano presenta diversos mecanismos que influyen en la propagación de señales. En el interior del mismo, debido a las características dieléctricas heterogéneas de los tejidos humanos (distintos valores de permitividad relativa $\varepsilon_{r}$ ) la señal sufre grandes pérdidas por absorción, reflexión entre capas de tejido y difracción. En el exterior, los principales fenómenos que influyen en la propagación son la reflexión de la señal transmitida en elementos del entorno, la difracción y el bloqueo de la señal por partes del cuerpo. Además, debido a que el cuerpo no es una entidad estática, el movimiento de este causa que el canal de propagación sea variable en el 
tiempo, pudiendo aparecer una variación relativa en la frecuencia de la señal recibida o efecto Doppler debida al cambio en la distancia relativa entre dispositivos ubicados en el cuerpo. Así mismo, este efecto puede darse en enlaces entre dispositivos ubicados en el cuerpo y dispositivos externos (ya sean fijos o móviles) por el movimiento de estos o por el movimiento de elementos presentes en las inmediaciones.

Debido a la influencia del cuerpo en el canal radio, el modelado de los diferentes escenarios de propagación correspondientes a los posibles enlaces entre dispositivos que conforman una WBAN es complejo. De manera general, nos interesa obtener un modelo estadístico que sea por un lado lo suficiente sencillo y por otro lo más detallado posible para ajustarse al máximo a los fenómenos observados. Además, hay que tener en cuenta que, ya que el cuerpo está en movimiento, las condiciones del canal varían en el tiempo.

El estudio de la propagación en la banda de UWB ha centrado gran parte de la atención en la comunidad científica durante años pero no ha sido hasta hace poco tiempo cuando, debido al creciente interés en el campo de las redes de área personal y corporal, se ha empezado a replantear los resultados existentes teniendo en cuenta la influencia que ejerce el cuerpo en el canal radio. Dependiendo de la ubicación de los dispositivos en relación con el cuerpo, se puede establecer la siguiente clasificación de los mismos:

- Dispositivos vestibles (wearables): son aquellos diseñados para ser colocados sobre el cuerpo, bien empleando un mecanismo de sujeción específico, bien por medio de la incorporación de los mismos a la estructura de una prenda de vestir.

- Dispositivos implantados: son aquellos diseñados para ser colocados ya sea de manera quirúrgica, o de manera no invasiva (por ejemplo ingeridos), en el interior del cuerpo humano.

Dependiendo de las distintas ubicaciones entre dispositivos que conforman una WBAN, se puede establecer la siguiente clasificación general de posibles tipos de canales de propagación [Hall y Hao, 2012]:

- In-body: canal radio entre dispositivos, alguno de los cuales se encuentra ubicado en el interior del cuerpo.

- On-body: canal radio entre dispositivos ubicados en la superficie del cuerpo, en contacto directo con el mismo o a una pequeña separación.

- Off-body: canal radio entre un dispositivo colocado en la superficie del cuerpo y otro ubicado en el entorno de este a una cierta distancia, normalmente inferior a $3 \mathrm{~m}$. Como extensión de este se puede considerar el canal de propagación entre dos 
dispositivos colocados en la superficie del cuerpo de dos individuos o canal Body-to-Body [Maman et al., 2014].

A pesar de que la anterior clasificación nos permite hacer una separación en dominios aislados (dentro del cuerpo, sobre el cuerpo y fuera del cuerpo), cada uno de ellos mostrando un comportamiento característico en cuanto a los fenómenos que afectan a la propagación de señales, no abarca todos los escenarios de comunicaciones posibles entre dispositivos en una WBAN. Por este motivo, se puede extender la clasificación de canales anterior de la siguiente forma:

- Implante a implante (IB2IB).

- Implante a superficie (IB2OB).

- Implante a exterior (IB2OFF).

- Superficie del cuerpo a superficie del cuerpo con visión directa (OB2OB LOS).

- Superficie del cuerpo a superficie del cuerpo sin visión directa (OB2OB NLOS).

- Superficie del cuerpo a exterior con visión directa (OB2OFF LOS).

- Superficie del cuerpo a exterior sin visión directa (OB2OFF NLOS).

Estos siete escenarios son los mismos que identifica el estándar IEEE 802.15.6. En este, a su vez, se plantean cuatro tipos de modelos de canal (CM, Channel Model) diferentes aplicables a los escenarios anteriores, nombrados de CM1 a CM4 tal y como se muestra en la Tabla 2.7.

\begin{tabular}{|c|c|c|c|}
\hline Escenario & Descripción & Banda de Frecuencias & $\begin{array}{l}\text { Modelo } \\
\text { de canal }\end{array}$ \\
\hline S1 & Implante a Implante & $402-405 \mathrm{MHz}$ & CM1 \\
\hline $\mathrm{S} 2$ & Implante a superficie & $402-405 \mathrm{MHz}$ & CM2 \\
\hline S3 & Implante a exterior & $402-405 \mathrm{MHz}$ & CM2 \\
\hline S4 & $\begin{array}{l}\text { Superficie a superficie } \\
\text { (LOS) }\end{array}$ & $\begin{array}{c}13.5,50,400,600,900 \\
\mathrm{MHz} 2.4 \\
3.1-10.6 \mathrm{GHz}\end{array}$ & CM3 \\
\hline S5 & $\begin{array}{l}\text { Superficie a superficie } \\
\text { (NLOS) }\end{array}$ & $\begin{array}{c}13.5,50,400,600,900 \\
\mathrm{MHz} 2.4 \\
3.1-10.6 \mathrm{GHz}\end{array}$ & CM3 \\
\hline S6 & $\begin{array}{c}\text { Superficie a exterior } \\
\text { (LOS) }\end{array}$ & $\begin{array}{l}900 \mathrm{MHz} 2.4, \\
3.1-10.6 \mathrm{GHz} \\
\end{array}$ & CM4 \\
\hline S7 & $\begin{array}{c}\text { Superficie a exterior } \\
\text { (NLOS) }\end{array}$ & $\begin{array}{l}900 \mathrm{MHz} 2.4, \\
3.1-10.6 \mathrm{GHz}\end{array}$ & CM4 \\
\hline
\end{tabular}

Tabla 2.7: Escenarios de interconexión entre dispositivos y modelos de canal [IEEE P802.15-08-078009-0006, 2009]. 
En la Figura 2.9. se identifican gráficamente los modelos de canal listados en la Tabla 2.7 .

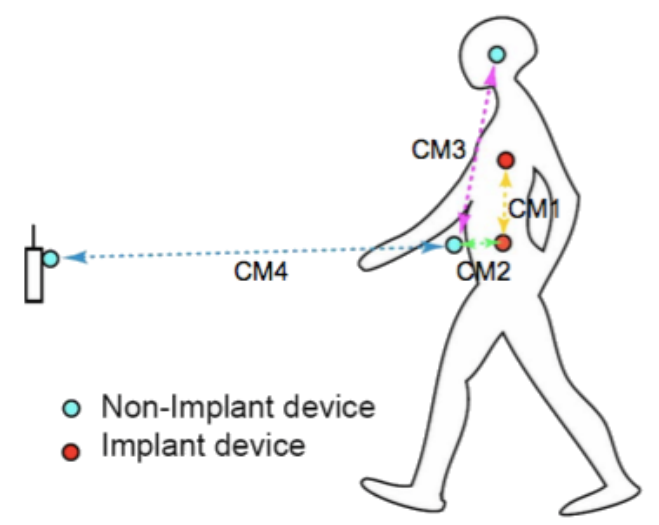

Figura 2.9: Canales de propagación en BAN [IEEE P802.15-08-0780-09-0006, 2009].

La propagación de señales en cada uno de los canales identificados anteriormente presenta unas particularidades que deben ser modeladas adecuadamente con el fin de diseñar sistemas de comunicaciones que operen de manera óptima bajo la influencia del cuerpo humano a la frecuencia considerada.

\subsubsection{Propagación multicamino}

Durante la propagación entre transmisor $(\mathrm{Tx})$ y receptor $(\mathrm{Rx})$, una señal puede interactuar con los diferentes elementos dispersores, reflectores, difractores, etc., presentes en el canal radio dando lugar a que la señal recibida esté formada por réplicas de la señal transmitida atenuadas, retardadas en el tiempo y posiblemente distorsionadas. A cada una estas réplicas se le denomina componente multicamino (MPC, Multipath Component). Debido a que habitualmente en una red de área corporal inalámbrica tanto transmisor y receptor como los diferentes elementos en el canal no se encuentran estáticos, el número y las características de cada componente multicamino varían en el tiempo de manera que, en general, el canal será percibido como variable en el tiempo.

En el caso de sistemas de banda estrecha, debido a la baja resolución temporal ( $\Delta \tau \approx 1 / B W$ ), no es posible identificar de manera precisa cada una de las MPC recibidas, por lo que la señal recibida resultante es la suma vectorial de cada una de ellas [Rappaport, 1996]. Esto provoca desvanecimiento (fading) en la amplitud de la señal recibida que puede ser más o menos profundo dependiendo de cómo se combinen en fase cada componente recibida. Además, considerando que el número de componentes es elevado, se puede asumir 
que se cumple el teorema del límite central y la amplitud de la señal resultante se puede modelar por medio de una estadística gaussiana o normal.

Al contrario de lo que sucede en los sistemas de banda estrecha, debido al gran ancho de banda disponible en UWB la resolución temporal es del orden de nanosegundos, lo que da lugar a poder identificar MPC con separaciones espaciales por bajo del centímetro. Esto se traduce en que el receptor es capaz de identificar componentes individuales, combinándolas (por ejemplo mediante un receptor Rake), reduciendo el efecto del desvanecimiento o fading en la señal recibida.

En el caso particular de la propagación en entornos centrados en el cuerpo, las componentes multicamino, tal y como se identifican en la Figura 2.10, pueden clasificarse en dos grupos:

- Componentes debidas a los fenómenos de difracción, reflexión, dispersión, etc., en partes del cuerpo.

- Componentes debidas a la interacción con el entorno.

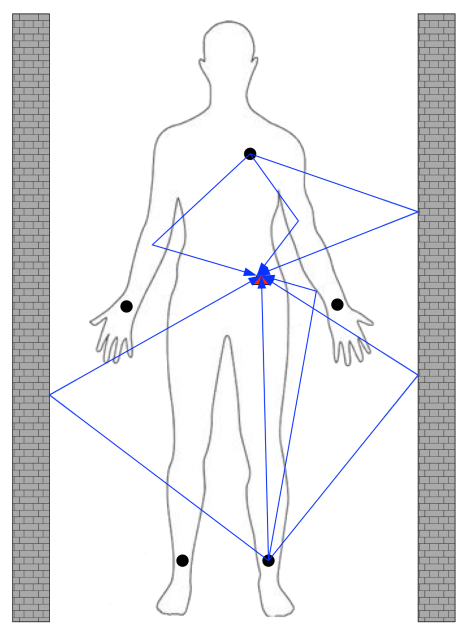

Figura 2.10: Propagación multicamino en WBAN.

Debido a la baja profundidad de penetración $\delta$ de las señales en el cuerpo para frecuencias por encima de $1 \mathrm{GHz}$, la propagación entre dos dispositivos ubicados en posiciones opuestas del cuerpo (NLOS), como por ejemplo uno colocado en la parte frontal y otro en la posterior, se produce principalmente por onda de superficie (creeping wave) y reflexión/difracción en partes del cuerpo y con elementos del entorno. Dependiendo de la aplicación concreta se considerarán predominantes unos efectos u otros, de manera que por ejemplo, en la caracterización de la propagación sobre el cuerpo serán de interés las componentes multicamino recibidas con retardos de unos pocos nanosegundos (ns) debidas al 
efecto del cuerpo, mientras que en la caracterización de la propagación entre elementos situados en el cuerpo y elementos externos (ya sean ubicados sobre el cuerpo de otra persona o fijos) será de interés además, la identificación de las componentes por reflexiones en el entorno.

\subsubsection{Modelado del canal de propagación WBAN en UWB}

Los efectos derivados de la interacción con el cuerpo por parte de los dispositivos que componen una WBAN, hacen que la propagación de señales en este tipo de canales radio presente características diferenciadoras respecto al resto de canales tradicionales. Como consecuencia de que las características de radiación de las antenas se vean afectadas por la proximidad con los tejidos biológicos, es muy difícil desacoplar el efecto de estas sobre las características de propagación del canal radio de manera que, habitualmente la caracterización de la propagación en canales WBAN incluye a las propias antenas como parte del canal radio. Aunque esto facilita el proceso de modelado, liga los resultados obtenidos de la caracterización de la propagación a las propiedades de las antenas empleadas durante las medidas.

A la hora de caracterizar los fenómenos asociados a la propagación de señales UWB en el canal WBAN, habitualmente se plantea un estudio de los parámetros del canal radio en base a dos modelos:

- Modelo de pérdidas de propagación.

- Modelo de perfil de potencia-retardo.

Los dos niveles de modelado anteriores permiten obtener una visión del canal de propagación WBAN en términos del comportamiento a gran y pequeña escala. Mientras que por medio del modelado de las pérdidas de propagación es posible determinar la variación a gran escala en la potencia media recibida en función de la distancia y la frecuencia, el modelado del perfil de potencia-retardo permite obtener una visión a pequeña escala de los efectos causados por la propagación multicamino, de especial importancia en sistemas UWB por la alta resolución temporal (y espacial) que presentan.

\subsubsection{Modelado de las pérdidas de propagación}

Las pérdidas de propagación (PL, Path Loss) representan la reducción en la potencia de una señal transmitida al propagarse en un cierto medio. La variación en la potencia media recibida presenta una doble dependencia: frecuencia y distancia, aunque habitualmente la 
dependencia de la frecuencia se suele ignorar considerando únicamente la dependencia con la distancia. En el caso del modelado de las pérdidas según la distancia, habitualmente se emplea el modelo tradicional que caracteriza el aumento lineal de las pérdidas con el logaritmo de la distancia en función de un determinado exponente de pérdidas $\gamma$ o $n$. Por otro lado, el efecto de dispersión del valor las pérdidas respecto al valor medio se modela por medio de un término de shadowing $S$ que tiene en cuenta el bloqueo de la señal por partes del cuerpo o elementos del entorno entre transmisor y receptor. En el Apéndice A, sección A.2.1.1 se detalla la metodología empleada para el modelado de las pérdidas de propagación.

Aunque en sistemas de banda estrecha la variación en las pérdidas de propagación debida a la variación en la frecuencia se suele ignorar, debido al ancho de banda de las señales en UWB, este efecto tiene especial relevancia en las WBAN debido a que las características dieléctricas de los tejidos varían en función de la frecuencia, por lo tanto la propagación de las señales tendrá unas consideraciones específicas en cada uno de los posibles enlaces entre dispositivos de una red de área corporal inalámbrica identificados en la Tabla 2.7. A continuación se detalla, para los tres canales genéricos: on-body, off-body e inbody, algunas de las principales características que afectan a las pérdidas de propagación en cada uno de ellos.

\section{A. Canales on-body}

En la banda de frecuencias disponibles en UWB de 3.1-10.6 GHz, la profundidad de penetración $\delta$ de las señales en el cuerpo es muy pequeña (del orden de milímetros). Por este motivo la propagación de señales enlaces on-body se produce mayoritariamente por onda de superficie (creeping wave). En este caso, la distancia recorrida por una señal entre dos dispositivos ubicados en posiciones opuestas del cuerpo es habitualmente considerada como la comprendida sobre la superficie del mismo y no la distancia directa entre ambos (a través del cuerpo) [Zasowski et al., 2005]. Durante la propagación de señales sobre el cuerpo, las señales sufren además pérdidas por difracción en la distintas partes de este.

Para entornos indoor, se han reportado valores de $n$ entre 2.8 para LOS y 1.34 para LOS [Abbasi et al., 2011], 2.9 para LOS y 1.7 para NLOS [Goswami et al., 2015] aunque en otros estudios se han reportado valores de 3, 4.4 [Ghannoum et al., 2010] y superiores a 8 [Takizawa et al., 2008] para LOS. Los valores para $n$ reportados en estudios realizados en entornos de cámara anecoica son significativamente superiores, entre otros motivos debido a la ausencia de componentes multicamino, obteniéndose valores en algunos casos por encima de 19 [Chen et al., 2011]. 


\section{B. Canales off-body}

Al contrario de lo que sucede en el caso de los canales on-body, el modelado de las pérdidas de propagación en enlaces off-body carece de un número significativo de contribuciones científicas. Las dos situaciones generales que se pueden dar en este tipo de enlaces son: visión directa (LOS) entre Tx y Rx o bloqueo de la señal entre ambos (NLOS).

Suponiendo una separación suficiente de la antena colocada sobre el cuerpo respecto a la superficie del mismo ( $>5 \mathrm{~mm}$ ), de manera que se minimicen los efectos de este sobre las características de propagación de la antena, las pérdidas que sufre la señal vienen dominadas por la distancia entre Tx y Rx, así como los efectos de reflexión, difracción y dispersión ocasionados por la interacción de la señal transmitida con los elementos del entorno.

\section{Canales in-body}

La propagación de señales en el interior del cuerpo es difícil de modelar. El cuerpo humano se suele considerar como un material dieléctrico con pérdidas cuyas características varían con la frecuencia. A frecuencias de UWB las pérdidas de propagación debido a las altas pérdidas de propagación entre capas de tejido son muy altas, por lo que como consecuencia, las frecuencias hábiles para el establecimiento de enlaces entre implantes o entre implantes y la superficie (o exterior) haciendo uso de la banda de UWB se restringen a la banda baja (3.1-4.8 GHz).

A partir del modelado electromagnético de los tejidos (ver Apéndice B), es posible cuantificar las pérdidas de propagación en función de la profundidad de penetración $\delta$, definida como la distancia a la cual la intensidad de campo de una señal incidente cae a $1 / e$ de su valor inicial, a las frecuencias consideradas. En la Figura 2.11. se muestra el valor de las pérdidas de propagación en tejidos de alto contenido en agua (como los músculos) según la frecuencia para dos profundidades: $5 \mathrm{~cm}$ y $10 \mathrm{~cm}$.

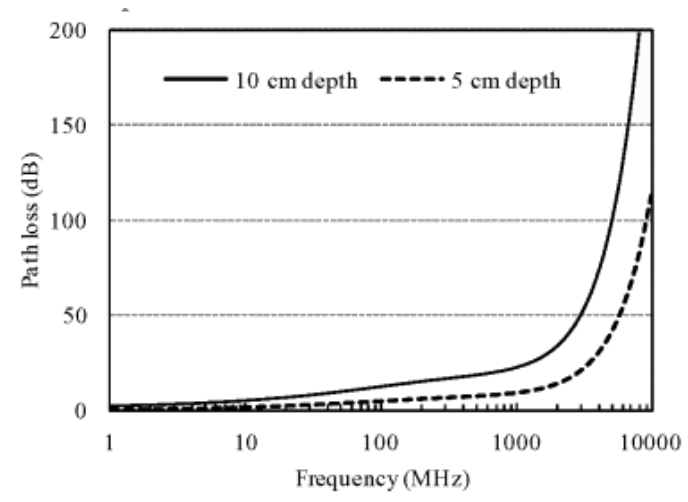

Figura 2.11: Dependencia de las pérdidas de propagación según la frecuencia en tejidos con alto contenido en agua [Wang y Wang, 2013]. 
Como se puede observar, las pérdidas para la banda de UWB son superiores a $20 \mathrm{~dB}$ a $3.1 \mathrm{GHz}$, haciendo inviable los enlaces por encima de $5 \mathrm{~cm}$ para frecuencias de la franja superior de la banda. Por medio de simulaciones se han reportado valores para el exponente de pérdidas de 0.85 [Khaleghi et al., 2011] para una profundidad del implante de 20-80 mm.

\subsubsection{Modelado del perfil de potencia-retardo (PDP)}

Como consecuencia de los fenómenos de interacción de una señal transmitida con el cuerpo y el entorno, tales como la reflexión, la difracción y la dispersión, al receptor llegará una composición de réplicas de la señal original, retardadas, atenuadas y, posiblemente, distorsionadas. Por esta razón, la respuesta al impulso variable temporal $h(t, \tau)$ del canal UWB puede modelarse de la siguiente forma [Pahlavan y Levesque, 2005]

$$
h(t, \tau)=\sum_{k} \beta_{k}(t) e^{j \phi_{k}(t)} \delta\left(t-\tau_{k}(t)\right)
$$

donde $k$ es el índice de cada componente multicamino, $\beta_{k}(t)$ es la ganancia compleja, $\phi_{k}(t)=2 \pi f_{c} \tau_{k}(t)+\phi_{0 k}$ es el desplazamiento en fase debido a propagación en espacio libre de la componente $k, \tau_{k}$ es el retardo de propagación dependiente de la longitud del camino recorrido entre Tx y $\mathrm{Rx}$, y $\delta(\cdot)$ es la función delta de Dirac. En este modelo, la variable $t$ representa el instante temporal al cual se refiere la realización de la respuesta al impulso, mientras que $\tau$ representa el retardo temporal de cada componente multicamino en referencia al instante $t$ tal y como se representa en la Figura 2.12.

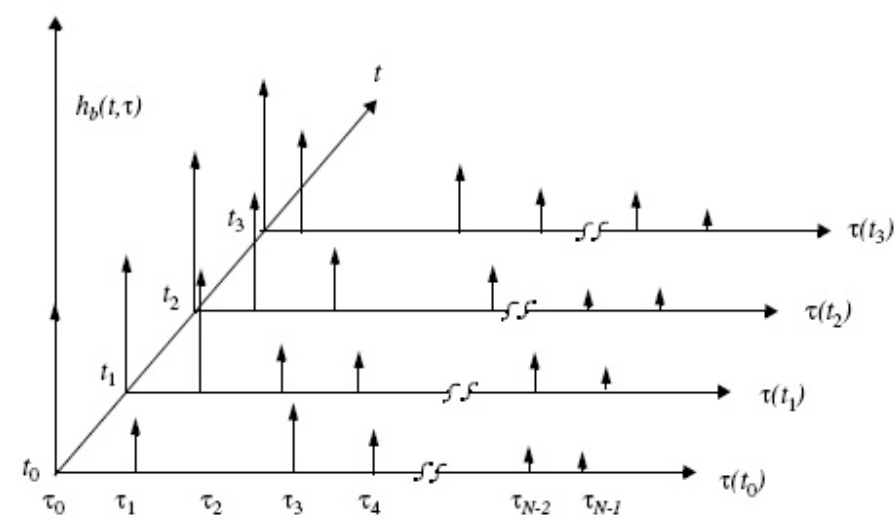

Figura 2.12: Modelo de respuesta al impulso variable temporal en un canal multicamino [Rappaport, 1996]. 
En este modelo, el eje de retardo se suele subdividir en intervalos de anchura equivalente a la resolución de la medida $(\Delta \tau \approx 1 / B W)$ denominados bins. En cada bin puede haber ninguna, una o múltiples componentes multicamino para un cierto valor de realización $t$. Por lo tanto, la capacidad de resolver componentes multicamino de un sistema vendrá dado por el ancho de banda ( $B W$ ) del mismo. En el caso de los sistemas UWB, considerando un ancho de banda total de $7.5 \mathrm{GHz}$ (suponiendo útil la banda completa de 3.1-10.6 GHz), la anchura de cada bin es aproximadamente 133 ps, lo que supone una capacidad de resolución espacial de unos $4 \mathrm{~cm}$. Las componentes con mismo retardo, que por lo tanto caen en un mismo bin, no pueden ser resueltas por el sistema y pueden dar lugar a desvanecimiento cuya estadística de amplitudes puede modelarse por medio de una determinada función de distribución. Habitualmente las distribuciones Rayleigh (en el caso de no existir una componente dominante) y Rice (en el caso de una componente dominante) son las que se emplean para modelar la estadística de desvanecimiento en los canales de propagación móviles. En el caso de las redes WBAN, múltiples estudios han determinado que la distribución log-normal es la que mejor caracteriza este tipo de desvanecimiento [Taparugssanagorn et al., 2009], [Roblin, 2011], [Fort et al., 2006b].

El modelado del efecto causado en el canal por la propagación multicamino se suele realizar por medio del análisis de la respuesta al impulso del canal $h(t, \tau)$ considerando este bajo condiciones de estacionalidad $(t=0)$. De esta manera se puede obtener una representación de la distribución de potencias de las componentes multicamino recibidas a diferentes retardos. A esta representación se le denomina perfil de potencia-retardo (PDP, Power Delay Profile). Del análisis del perfil de potencia-retardo se puede derivar la caracterización estadística en términos de los momentos centrales de primer y segundo orden. El análisis detallado se puede consultar en el Apéndice A, sección A.2.1.2.

\subsubsection{Modelos estadísticos de canal WBAN en UWB}

A la hora de platear un modelo estadístico que describa la propagación de señales en un canal WBAN para la banda de UWB, habitualmente se emplean modificaciones de modelos de propagación multicamino previamente desarrollados para esta banda, principalmente del modelo Saleh-Valenzuela (S-V) [Saleh y Valenzuela, 1987], [Molisch, 2009]. Debido a la alta resolución temporal disponible en la banda de UWB, la señal recibida está compuesta por un conjunto de réplicas de la señal transmitida agrupadas en clusters cuya estructura depende del entorno físico de propagación. En el caso de propagación en WBAN, algunos estudios consideran únicamente dos clusters, uno debido a las componentes recibidas por fenómenos derivados de la interacción de la señal transmitida con partes del cuerpo y otro 
formado por las componentes multicamino debidas a la interacción con el entorno [Roblin, 2011]. A pesar de esto, en la propuesta de modelos de canal para el estándar IEEE 802.15.6 [IEEE P802.15-08-0780-09-0006, 2009] los modelos detallados para la banda de UWB consideran las diferentes componentes multicamino agrupadas en un único cluster. Debido a que esta banda únicamente se considera para la propagación de señales sobre el cuerpo (canales on-body) o entre el cuerpo y un dispositivo externo (canales off-body), los modelos de canal para los cuales se presenta una caracterización de la respuesta al impulso son el CM3 y el CM4.

El modelo de canal CM3 propuesto en el estándar IEEE 802.15.6 supone una adaptación del S-V considerando un único cluster. Por lo tanto, la respuesta al impulso $h(\tau)$ se modela de la siguiente forma [IEEE P802.15-08-0780-09-0006, 2009],

$$
\begin{gathered}
h(\tau)=\sum_{l=0}^{N-1} a_{l} e^{\left(j \phi_{l}\right)} \delta\left(\tau-\tau_{l}\right) \\
10 \log _{10}\left|a_{l}\right|^{2}=\left\{\begin{array}{c}
0 \quad l=0 \\
\gamma_{0}+10 \log _{10}\left(\mathrm{e}^{\left(-\frac{\tau_{l}}{\Gamma}\right)}\right)+s \quad l \neq 0
\end{array}\right. \\
p\left(\tau_{l} \mid \tau_{l-1}\right)=\lambda \mathrm{e}^{\left(-\lambda\left(\tau_{l}-\tau_{l-1}\right)\right)} \\
p(L)=\frac{\bar{L}^{L} \exp (\bar{L})}{L !}
\end{gathered}
$$

donde:

$a_{l}$ es la amplitud de la componente multicamino l-ésima.

$\tau_{l}$ es el retardo correspondiente a la componente multicamino l-ésima.

$\phi_{l}$ es la fase de la componente multicamino l-ésima y se modela con una distribución uniforme entre $[0,2 \pi)$.

$L$ es el número total de componentes multicamino consideradas.

$\delta(\cdot)$ es la función delta de Dirac.

$\Gamma$ es un parámetro de decaimiento exponencial con factor Rice $\gamma_{0}$. 
$S$ es un factor de shadowing, modelado mediante una distribución normal de medio 0 y desviación típica $\sigma_{s}\left(N\left(0, \sigma_{s}\right)\right)$.

$\lambda$ es la tasa de llegada de componentes multicamino asociada a la distribución de Poisson que modela la llegada de componentes $p\left(\tau_{l} \mid \tau_{l-1}\right)$.

$\bar{L}$ es el número medio de componentes multicamino recibidas.

En la Figura 2.13. se representa cada uno de los parámetros del modelo de respuesta al impulso descrito arriba.

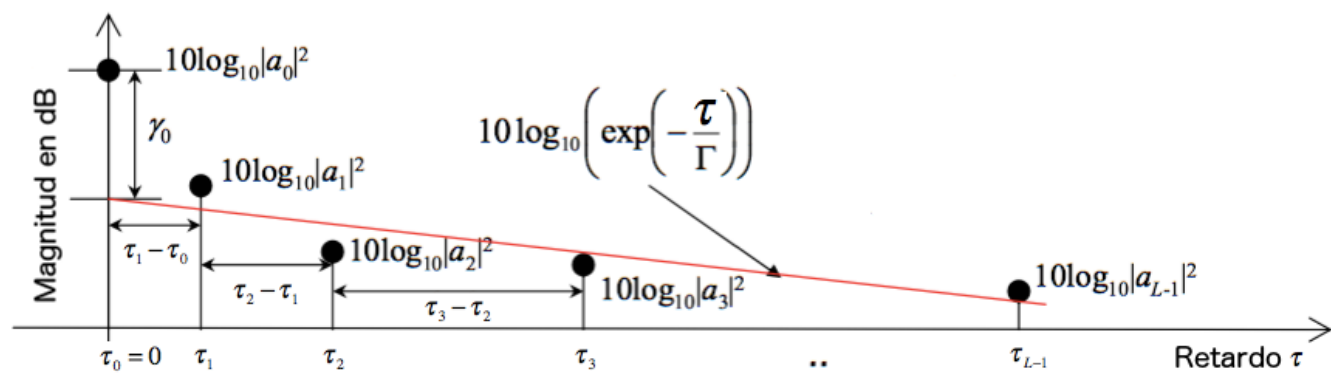

Figura 2.13: Modelo de respuesta al impulso para el canal CM3 en el estándar IEEE 802.15.6.

$\mathrm{Al}$ igual que el modelo anterior, el modelo de canal CM4 para off-body considera una adaptación del S-V con un único cluster. Por lo tanto, la respuesta al impulso $h(\tau)$ se modela de la siguiente forma [IEEE P802.15-08-0780-09-0006, 2009],

$$
\begin{aligned}
& h(\tau)=\sum_{l=0}^{L-1} a_{l} \delta\left(\tau-\tau_{l}\right) \\
& \left|a_{l}\right|^{2}=\Omega_{0} e^{-\frac{\tau_{l}}{\Gamma}-k[1-\delta(l)]} \beta
\end{aligned}
$$

donde:

$\bar{L}$ es el número medio de componentes multicamino recibidas, modelado por medio de una distribución de Poisson.

$a_{l}$ es la amplitud de la componente multicamino l-ésima.

$\tau_{l}$ es el retardo correspondiente a la componente multicamino l-ésima, modelado por medio de una distribución de Poisson.

$k$ es el efecto del factor $\mathrm{K}$ de la distribución Rice, calculado como $k=\Delta k(\ln 10 / 10)$. 
$\Omega_{0}$ son las pérdidas de propagación. En el caso de normalizar la amplitud de la respuesta al impulso, se puede considerar $\Omega_{0}=1$.

$\delta(\cdot)$ es la función delta de Dirac.

$\Gamma$ es un parámetro de decaimiento exponencial.

$\beta$ es un factor de shadowing, modelado mediante una distribución normal de medio $0 \mathrm{y}$ desviación típica $\sigma_{\beta}\left(N\left(0, \sigma_{\beta}\right)\right)$. 


\section{Capítulo 3 \\ Caracterización Estática del Canal Off-Body}

En este capítulo se presentan los resultados obtenidos de la caracterización del canal de propagación off-body en la banda UWB de 3.1-8 GHz entre un dispositivo colocado en la superficie del cuerpo y otro externo, a modo de punto de acceso, a una distancia máxima de $3 \mathrm{~m}$, considerando el canal radio estático y visión directa (LOS). El análisis se ha realizado a partir de medidas experimentales del canal radio considerando diferentes sujetos y entornos.

En primer lugar, se exponen los resultados del estudio realizado con el objetivo de evaluar la influencia que el entorno de medidas tiene sobre el canal de propagación off-body entre una antena transmisora fija y una receptora colocada sobre el cuerpo de un sujeto. Adicionalmente, los datos se han comparado con los valores obtenidos en condiciones de propagación sin la influencia del sujeto.

En segundo lugar, se presentan los resultados relativos a la caracterización del canal de propagación off-body en términos de las pérdidas de propagación y la dispersión temporal, considerando diferentes enlaces entre una antena receptora colocada en distintas partes del cuerpo de un sujeto y una antena transmisora fija.

Por último, la variación en el canal de propagación off-body relativa a la postura de un sujeto es evaluada considerando dos posiciones: de pie y tumbado. La caracterización del canal off-body se presenta por un lado en términos de las pérdidas de propagación y dispersión temporal del canal de propagación y por otro lado en función de la variación de los parámetros del modelo de canal CM4 para UWB propuesto por en el estándar IEEE 802.15.6 [IEEE 802.15.6, 2012]. 


\subsection{Estado del arte}

La caracterización del canal de propagación off-body entre un dispositivo colocado sobre la superficie del cuerpo de un sujeto y un punto de acceso externo (AP), en la banda de UWB es un campo poco tratado en la literatura, si se compara con el volumen de estudios existentes relacionados con el canal on-body entre dos dispositivos colocados sobre la superficie del cuerpo como los incluidos en [Fort, 2006] para la banda de 3-6 GHz, en [Chen et al., 2011] para 3-5 GHz, en [Hirose et al., 2012] para la banda completa regulada por la FCC de 3.1-10.6 GHz y en [See et al., 2012] para 3.5-4.5 GHz.

El estudio de la propagación en el canal off-body se realiza mayoritariamente desde la perspectiva del modelado de las pérdidas de propagación y el comportamiento dispersivo temporal del canal [Abbasi et al., 2012], y en pocos casos se presenta un modelo completo de respuesta al impulso del canal. En [Goulianos et al., 2010] se detalla un modelo de PDP para la banda de 3.5-6.5 GHz, en [Yu et al., 2016] un modelo autoregresivo (AR, Autoregressive) de respuesta al impulso para la banda de 6-8.5 GHz y en [Di Bari et al., 2013] se presenta una validación estadística del modelo de respuesta al impulso para el CM4 del IEEE 802.15.6 en la banda de 3-10 GHz considerando 109 posiciones de colocación de una antena receptora sobre el cuerpo de un sujeto.

Habitualmente, el modelado de la propagación en redes WBAN y en particular para el caso del canal off-body, se lleva a cabo desde una perspectiva estadística a partir de la información acerca del canal radio obtenida ya sea mediante la medida de la respuesta en frecuencia del canal por medio de un analizador de redes vectorial o VNA (medida del canal radio en frecuencia), o la respuesta al impulso por medio de un osciloscopio digital (medida del canal radio en el tiempo) [Catherwood y Scanlon, 2013]. En algunos estudios, el análisis de la propagación se lleva a cabo a partir de los datos acerca de la variación de la potencia recibida (RSS, Received Signal Strength) empleando dispositivos comerciales tales como los PulseOn ${ }^{\mathrm{TM}}$ de TimeDomain ${ }^{\circledR}$ como en [Khan et al., 2013]. Este último método, aunque permite caracterizar el desvanecimiento de la señal recibida empleando estadísticos de primer y segundo orden, ofrece una visión limitada del canal radio y por lo tanto, insuficiente para el diseño de sistemas de comunicaciones inalámbricos operando en entornos reales centrados en el cuerpo.

Los modelos estadísticos para off-body en UWB contemplados en los distintos estudios existentes se pueden clasificar a dos niveles: gran escala y pequeña escala. Los modelos a gran escala consideran tanto las pérdidas de propagación (Path Loss) como el shadowing o efecto de bloqueo de la señal por parte del cuerpo, mientras que los modelos a 
pequeña escala se centran en la caracterización del desvanecimiento o fading en el canal debido principalmente al efecto multicamino. El modelo de pérdidas de propagación más habitual en la literatura sobre WBAN, además de ser el contemplado en la propuesta de modelos de canal para el estándar IEEE 802.15.6 [IEEE P802.15-08-0780-09-0006, 2009] para UWB tanto en el modelo de canal CM3 como CM4, en base a los estudios presentados en [Aoyagi et al., 2008] y [Sawada et al., 2008], es el que contempla un aumento lineal de las pérdidas con el logaritmo de la distancia según un exponente $\gamma$, [Khan et al., 2011], [Abassi, 2012], [Yoo y Cotton, 2015]. Los valores reportados en diferentes estudios para el exponente de pérdidas van desde 1.07 para LOS y 1.09 para NLOS [Abbasi et al., 2011], 1.15 para LOS y 1.52 para NLOS [Khan et al., 2011], hasta 2.75 para LOS [Serna et al., 2015]. La alta disparidad en los valores obtenidos para los exponentes de pérdidas sugiere que estos son fuertemente dependientes de las medidas realizadas (tipo de antena, entorno, posición de colocación sobre el cuerpo, etc.). Además, los valores obtenidos para el exponente de pérdidas menores que 2 (condiciones de propagación ideales en espacio libre) ponen en duda que los métodos de estima de las pérdidas haciendo uso de las técnicas tradicionales basadas en la distancia entre Tx y Rx sean eficaces en su aplicación a las redes de área corporal [Smith et al., 2013]. En cuanto al modelado del shadowing o bloqueo de la señal transmitida por partes del cuerpo entre Tx y Rx, diferentes estudios han confirmado que la distribución log-normal es la que mejor se ajusta a la estadística de los valores observados [Wang y Wang, 2013].

La caracterización a pequeña escala del canal off-body en UWB se realiza a partir de la respuesta al impulso del canal $h(t, \tau)$. Habitualmente el eje de retardo se cuantifica en intervalos (bins) de tamaño aproximado a la inversa al ancho de banda considerado ( $\Delta \tau \approx 1 / B W$ ) [Molisch, 2009] cuya estadística de desvanecimiento puede modelarse por medio de una distribución log-normal, ya que debido a que, al contrario de lo que sucede en los sistemas de banda estrecha, el número de componentes multicamino recibidas con diferencia de retardos inferior al tamaño de cada bin es pequeño, no puede aplicarse el teorema del límite central y modelar el proceso mediante una distribución Rayleigh o Rice. A partir del perfil de potencia-retardo considerando el canal estacionario, calculado como $|h(\tau)|^{2}$, puede caracterizarse el comportamiento dispersivo temporal del canal, habitualmente determinado por medio dos parámetros: retardo medio (mean excess delay) y el valor rms del ensanchamiento de retardo (rms delay spread) (ver Apéndice A, sección A.2.1.2). Esto es de importancia fundamental en el diseño de sistemas de comunicaciones puesto que permite definir la máxima tasa de transferencia y el esquema de modulación óptimo. 
Los entornos estudiados principalmente son por un lado interiores (indoor), tales como oficina, laboratorio u hospital [Taparugssanagorn et al., 2009b], y por otro cámara anecoica [Abbasi et al., 2012], [Goulianos et al., 2008]. Además, los diferentes estudios consideran medidas tanto sobre sujetos reales como empleando materiales que emulan de manera sintética las características dieléctricas de los tejido biológicos en una banda de frecuencias [Pasquero y D’Errico, 2016].

Debido a la interacción en campo cercano con el cuerpo, las características de propagación de las antenas, tales como la frecuencia de resonancia, la impedancia de entrada y el diagrama de radiación [Edwards y Khattak, 2010] sufren variaciones. Además, conforme la antena está mas cerca del cuerpo, se ha observado un aumento en la directividad de la misma en la dirección normal a este. Así mismo, se ha observado una reducción en las pérdidas de camino cuando la distancia de separación con el cuerpo es igual a $5 \mathrm{~mm}$ [Ali et al., 2010] o superior [Tuovinen et al., 2013].

A lo largo de este capítulo se pretende por un lado realizar una caracterización de la influencia que el entorno y la posición de colocación de una antena sobre el cuerpo de un sujeto ejerce sobre las características del canal de propagación off-body entre una antena fija colocada a modo de punto de acceso en el entorno de un sujeto y una antena colocada sobre la superficie del cuerpo del mismo, y por otro lado validar el modelo de canal CM4 para UWB propuesto en el estándar IEEE 802.15.6. Para ello se han desarrollado un conjunto de medidas sobre sujetos reales en dos entornos de interior de diferentes dimensiones y características. 


\subsection{Esquema de medidas del canal de propagación off-body estático}

La caracterización de la propagación off-body en la banda de UWB entre un dispositivo Rx colocado en diferentes partes del cuerpo de varios sujetos y uno Tx situado a una distancia máxima de $3 \mathrm{~m}$ y a una altura de $1.4 \mathrm{~m}$, considerando el canal estático y visión directa entre ambos, se ha realizado en base a las medidas del canal radio por medio de un esquema de medidas en el dominio de la frecuencia. En la Figura 3.1 se describen los diferentes elementos empleados para realizar la medida del canal de propagación.

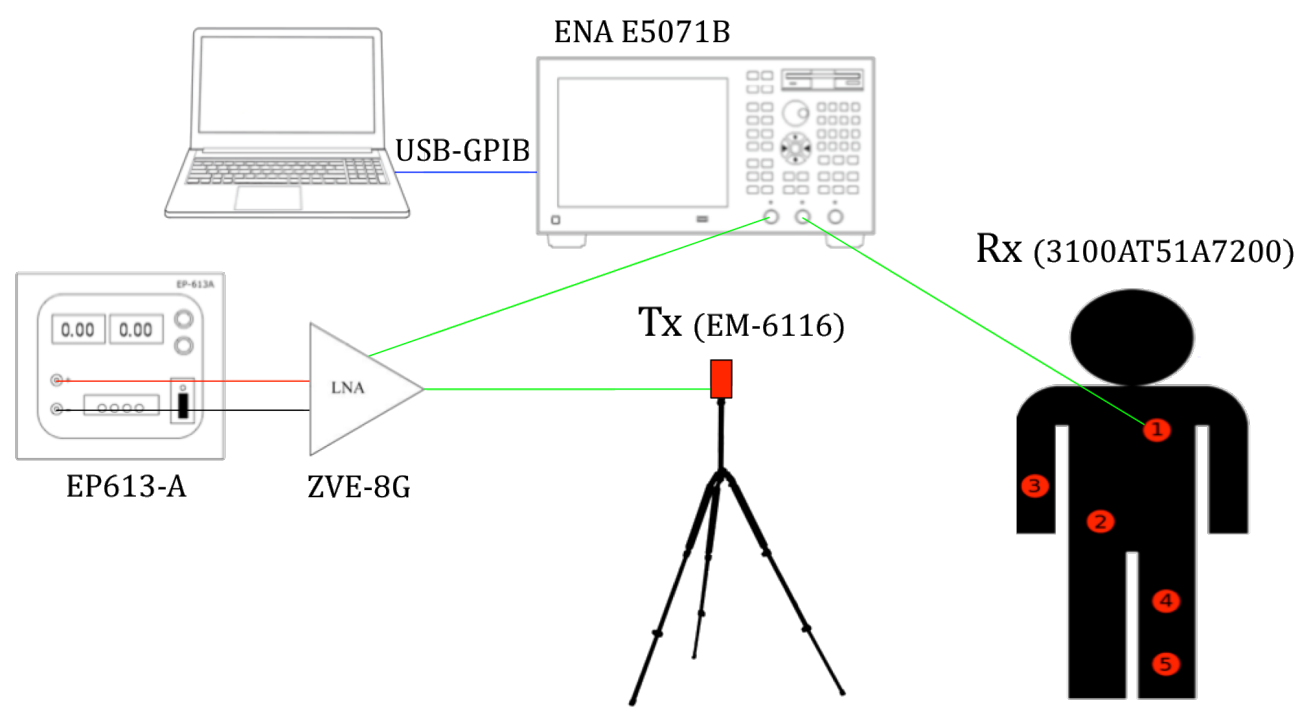

Figura 3.1: Esquema de medidas en frecuencia del canal off-body UWB.

Se ha empleado un analizador de redes vectorial (VNA, Vector Network Analyzer) Agilent ENA E5071B como elemento central para medir el parámetro de transmisión $S_{21}$ del canal, equivalente a la respuesta en frecuencia $H(f, t)$ del mismo [Rappaport, 1996]. Debido a que se han considerado condiciones de estacionalidad en sentido amplio y scattering incorrelado (WSSUS, Wide-Sense Stationary Uncorrelated Scattering) en el canal radio (ver Apéndice A, sección A.1.3), la respuesta en frecuencia anterior se puede suponer independiente del instante temporal de la realización $(t=0)$ y simplificarla a $H(f)$.

Para la realización de las medidas del canal radio, la salida del puerto de Tx del VNA se ha conectado mediante un cable de $1 \mathrm{~m}$ (con $2 \mathrm{~dB}$ de pérdidas) a la entrada de un amplificador Mini-Circuits ZVE-8G, el cual posee una banda de operación de 2-8 GHz, previamente alimentado a $12 \mathrm{~V}$ por medio de una fuente de alimentación de tensión variable modelo EP613-A. La salida del amplificador se ha conectado mediante un cable de $1 \mathrm{~m}$ a una antena UWB omnidireccional modelo EM-6116. Por otro lado, el puerto de entrada 1 del VNA se ha conectado por medio de un cable de $6 \mathrm{~m}$ (con $10 \mathrm{~dB}$ de pérdidas) con una antena 
UWB de bajo perfil modelo 3100AT51A7200 del fabricante Johanson Technology. Esta antena se ha colocado sobre diferentes partes del cuerpo de un conjunto de sujetos, elegidas por su idoneidad como punto de colocación de distintos dispositivos para aplicaciones médicas y no médicas. El control del proceso de medidas se ha realizado por medio de una aplicación desarrollada en Visual Basic 6.0 y ejecutada en un portátil conectado por medio de un interfaz USB-GPIB con el VNA. Tanto los detalles de los elementos empleados como el software puede consultarse en el Apéndice C.

Con el fin de eliminar la influencia de cables y resto de dispositivos empleados, se ha realizado un proceso previo de calibración de tipo Thru en el VNA. Para ello, por medio de un conector de unión se ha cerrado el circuito conectando el cable de la antena Tx con el de la antena Rx. Con este procedimiento, las pérdidas y retardos introducidos por los elementos de medida son eliminados pero no los posibles efectos introducidos por las antenas. Una vez completado el proceso de calibración, se ha procedido a medir el rango dinámico del sistema. Para ello se ha apagado la fuente de alimentación y se ha medido el parámetro $S_{21}$. El valor medio obtenido para el ruido ha sido en torno a $-106.52 \mathrm{~dB}$. En la Figura 3.2 se muestra el valor medido.

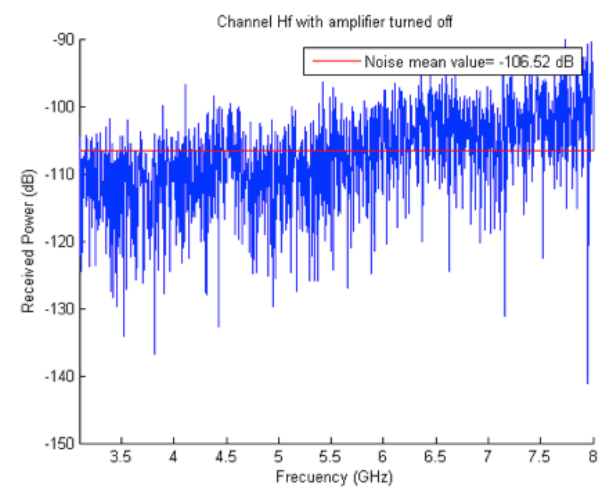

Figura 3.2: Medición del rango dinámico del sistema de medidas.

Ya que la frecuencia máxima de operación del amplificador empleado llega hasta los $8 \mathrm{GHz}$, las medidas se han limitado a la banda UWB de 3.1-8 GHz. Para ello, el analizador de redes se ha configurado con los parámetros indicados en la Tabla 3.1.

\begin{tabular}{|l|l|}
\hline Potencia Tx & $-6 \mathrm{dBm}$ \\
\hline Rango de medida & $3.1-8 \mathrm{GHz}$ \\
\hline Ancho de banda intermedio (IF) & $1 \mathrm{kHz}$ \\
\hline Número de puntos & 1601 \\
\hline Formato de datos & Polar/lineal \\
\hline Tiempo de medida & Automático \\
\hline
\end{tabular}

Tabla 3.1: Configuración del VNA para las medidas off-body UWB en estático. 
A partir de la configuración escogida para el VNA, el barrido en frecuencia por medio de la generación de 1601 tonos entre 3.1-8 $\mathrm{GHz}(B W=4.9 \mathrm{GHz})$ permite detectar componentes multicamino con un retardo máximo, $\tau_{\max }=(N-1) / B W$ de 326.5 ns. Además, la resolución temporal del sistema $\Delta \tau \approx 1 / B W$ es de aproximadamente, $0.2 \mathrm{~ns}$.

Con el fin de aumentar el rango dinámico de las medidas y eliminar los efectos producidos por pequeñas variaciones en la postura de los sujetos durante el tiempo de medida del canal radio, se han obtenido 10 realizaciones de cada una de las respuestas en frecuencia $H(f)$ para cada enlace $\mathrm{Tx}-\mathrm{Rx}$, con un tiempo total de medida inferior a 1 minuto, que posteriormente se han promediado temporalmente durante la etapa de procesado de datos previamente al análisis en banda ancha del canal radio.

\subsection{Metodología de medidas}

Se ha realizado un conjunto de medidas en frecuencia del canal radio, empleando los elementos descritos en el punto anterior, destinadas a la caracterización de diferentes aspectos de la propagación off-body en entornos de interior para la banda de UWB. Estas medidas se han llevado a cabo en dos entornos distintos y sobre sujetos reales. En los siguiente puntos se detallan las características tanto de los entornos en los que se han desarrollado las medidas como de los sujetos.

\subsubsection{Descripción de los entornos de medidas}

La caracterización de la propagación off-body se ha realizado a partir de las medidas en frecuencia del canal radio realizadas en dos entornos de interior diferentes situados en la planta sótano de la Escuela Técnica Superior de Ingeniería de Telecomunicación (ETSIT) de la Universidad Politécnica de Cartagena (UPCT). En la Figura 3.3 se muestra la localización de los emplazamientos donde se realizaron las medidas. 


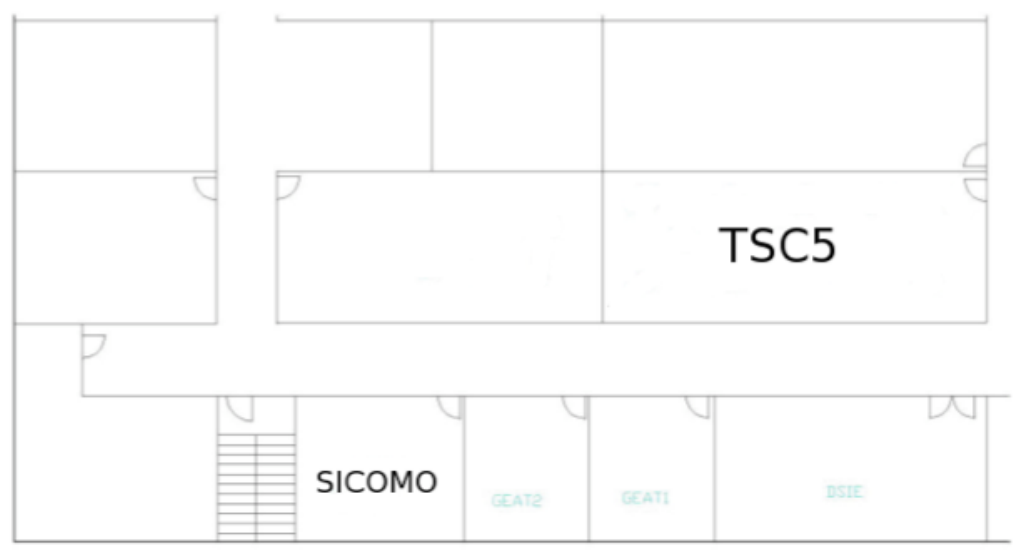

Figura 3.3: Plano de la planta sótano del edificio de la ETSIT.

El primero de los entornos es el laboratorio TSC5. Las medidas se realizaron en un área de 5.9 x $5.9 \mathrm{~m}$ situada al fondo del mismo y delimitada por elementos de mobiliario. Tanto las paredes como el suelo están construidas en hormigón y todo el perímetro está rodeado por mesas con elementos metálicos y equipos electrónicos. El techo se encuentra a una altura superior a $3 \mathrm{~m}$. En la Figura 3.4 se presenta un esquema del área de medidas en el interior del laboratorio.

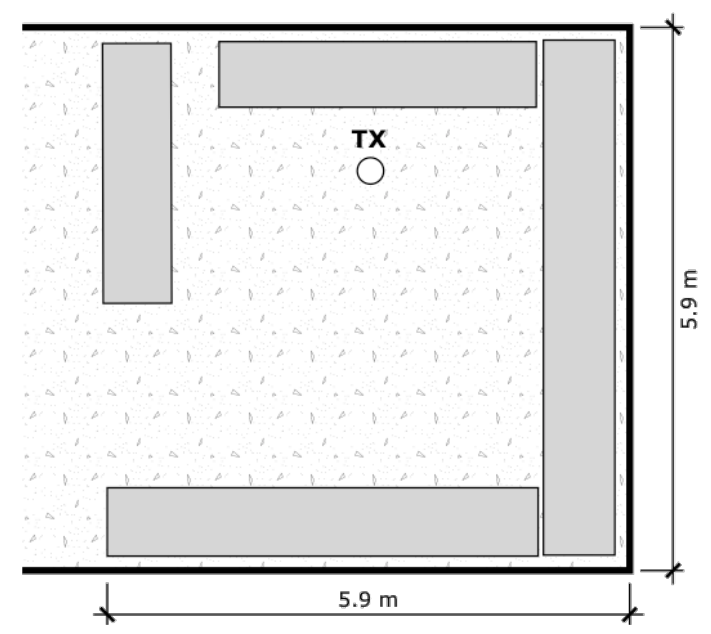

Figura 3.4: Esquema del entorno de medidas en el laboratorio TSC5.

El segundo de los entornos donde se han realizado las medidas es el laboratorio del grupo de Sistemas de Comunicaciones Móviles (SiCoMo). Se trata de una estancia de $5.7 \mathrm{~m}$ de ancho, $6.6 \mathrm{~m}$ de largo y una altura del techo superior a los $3 \mathrm{~m}$. Al igual que en el caso del laboratorio TSC5 el perímetro se encuentra rodeado por mesas de madera con elementos metálicos y ordenadores. Tanto el suelo y techo como las paredes están fabricadas en hormigón. En la Figura 3.5 se presenta un esquema del área de medidas en el interior del laboratorio del SiCoMo. 


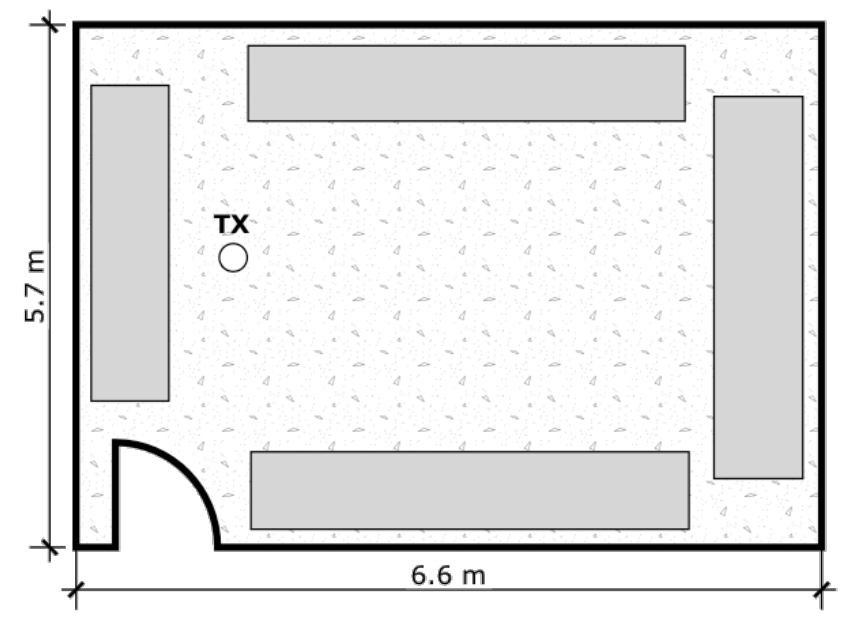

Figura 3.5: Esquema del entorno de medidas en el laboratorio del SiCoMo.

\subsubsection{Descripción de los sujetos}

Las medidas de la respuesta en frecuencia del canal estático off-body en la banda de UWB se han efectuado considerando una antena transmisora fija, a modo de punto de acceso, y una antena receptora colocada sobre diferentes partes del cuerpo de sujetos reales, situados estos a una distancia de separación mínima de $1 \mathrm{~m}$ respecto de la antena Tx. Considerando un valor máximo para el coeficiente de absorción específica (SAR), relativo a la exposición a campos electromagnéticos no ionizantes de alta frecuencia $(100 \mathrm{kHz}$ a $100 \mathrm{GHz})$, de $0.08 \mathrm{~W} / \mathrm{Kg}$ para cuerpo completo [ICNIRP, 2009] y considerando un peso medio de los sujetos de $70 \mathrm{Kg}$, se tiene un valor máximo de SAR de $5.6 \mathrm{~W}$ (para un tiempo máximo de exposición de 6 minutos). En el caso de las medidas realizadas, teniendo en cuenta una potencia de emisión de $-6 \mathrm{dBm}$ y el efecto del amplificador ( $+34 \mathrm{~dB}$ de ganancia) y las pérdidas de los cables, la potencia radiada estimada se encuentra entorno a $27 \mathrm{dBm}$, lo cual teniendo en cuenta además la distancia de separación mínima de $1 \mathrm{~m}$ entre la fuente emisora y los sujetos, garantiza un nivel de exposición muy inferior al máximo considerado como seguro.

En las medidas realizadas en el laboratorio TSC5 se han empleado dos sujetos con las características indicadas en la Tabla 3.2.

\begin{tabular}{|l|c|c|}
\hline & Sujeto 1 & Sujeto 2 \\
\hline Sexo & Hombre & Hombre \\
\hline Edad (años) & 33 & 34 \\
\hline Altura (cm) & 182 & 172 \\
\hline Peso (kg) & 82 & 65 \\
\hline
\end{tabular}

Tabla 3.2: Sujetos en medidas en laboratorio TSC5. 
Por otro lado, en las medidas realizadas en el laboratorio del SiCoMo se han empleado tres sujetos cuyas características se indican en la Tabla 3.3.

\begin{tabular}{|l|c|c|c|}
\hline & Sujeto 1 & Sujeto 2 & Sujeto 3 \\
\hline Sexo & Hombre & Mujer & Mujer \\
\hline Edad (años) & 34 & 23 & 23 \\
\hline Altura (cm) & 172 & 165 & 170 \\
\hline Peso (kg) & 65 & 54 & 73 \\
\hline
\end{tabular}

Tabla 3.3: Sujetos en medidas en laboratorio del SiCoMo.

Previo a la realización de las medidas se ha pedido a todos los sujetos que mantengan la postura estática en la posición considerada con el fin de evitar posibles variaciones en el canal de propagación durante el tiempo de medidas del mismo.

Las medidas del canal de propagación off-body se han llevado a cabo considerando cinco puntos de colocación sobre la parte frontal del cuerpo de los sujetos y bajo condiciones de visión directa (LOS). Las posiciones han sido seleccionadas por ser posibles puntos de colocación de dispositivos para aplicaciones medicas y no médicas. En la Figura 3.6 se muestra las diferentes posiciones consideradas.

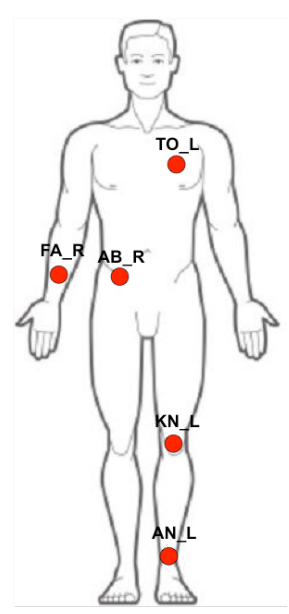

Figura 3.6: Posiciones de colocación de la antena Rx sobre el cuerpo de los sujetos.

\subsection{Análisis de los datos}

La caracterización del canal de propagación off-body UWB bajo condiciones estáticas presentada en este capítulo se ha realizado a partir de la caracterización estadística de los fenómenos a gran escala y pequeña escala del canal radio. Los parámetros considerados a gran escala son las pérdidas de camino (Path Loss) y el shadowing. Por otro lado, los parámetros considerados para la caracterización de los fenómenos a pequeña escala, centrados en los efectos derivados de la propagación multicamino, son aquellos asociados a la 
dispersión temporal producida por el canal. Adicionalmente, se han derivado los parámetros asociados al modelo de canal CM4 propuesto en el estándar IEEE 802.1.5.6 considerando como medio físico (PHY) UWB [IEEE P802.15-08-0780-09-0006, 2009].

Durante la etapa de procesado de los datos mediante Matlab, y salvo que se indique lo contrario, el conjunto de las 10 realizaciones de la respuesta en frecuencia del canal $H(f)$, para cada grupo de medidas, obtenidas por medio del VNA configurado este con los parámetros descritos en la Tabla 3.1, han sido primeramente promediadas temporalmente y el resultado convertido al dominio del tiempo para obtener la correspondiente respuesta al impulso del canal $h(\tau)$, calculada de la siguiente forma,

$$
h(\tau)=i f f t\left\{W_{\text {Hann }}\left(\frac{1}{10} \sum_{i=1}^{10} H_{i}(f)\right)\right\}
$$

donde $W_{\text {Hann }}$ es una ventana de Hann de longitud igual al total de puntos en frecuencia considerados (1601). El objetivo del enventanado es reducir los lóbulos laterales en la respuesta al impulso debido a que la respuesta en frecuencia es realmente una secuencia limitada en banda. Como inconveniente, el enventanado reduce la resolución temporal de la respuesta al impulso por un factor $\beta$ dependiente del ancho a $-3 \mathrm{~dB}$ del lóbulo principal del tipo de ventana empleado, en comparación con el de la ventana rectangular. En el caso de usar una ventana de Hann $\beta \approx 1.6$ por lo la resolución de la medida se reduce en el mismo valor a $\Delta \tau=\beta / B W(\Delta \tau \approx 1.6 \times 0.22 \mathrm{~ns} \approx 0.4 \mathrm{~ns})$.

Para cada respuesta al impulso, se ha obtenido el perfil de potencia-retardo o PDP (ver Apéndice A, sección A.2.1) de la siguiente forma,

$$
\operatorname{PDP}(\tau)=|h(\tau)|^{2}
$$

A partir del PDP se puede calcular las pérdidas de propagación como la inversa de la ganancia calculada en unidades logarítmicas a partir de la potencia de las componentes multicamino recibidas, de la siguiente forma (ver Apéndice A, sección A.2.1.1),

$$
P L(d)=-10 \log _{10}\left(\sum_{\tau} P D P(\tau)\right)
$$


Las pérdidas de propagación experimentadas por una señal transmitida se han modelado por medio de una ley creciente en función del logaritmo de la distancia con exponente $\gamma$ de la siguiente forma,

$$
P L_{d B}(d)=P L_{d B}\left(d_{0}\right)+10 \gamma \log _{10}\left(\frac{d}{d_{0}}\right)+S_{\sigma}
$$

donde $P L_{d B}\left(d_{0}\right)$ son las perdidas a una distancia de referencia, que a lo largo de la presente tesis hemos fijado a $1 \mathrm{~m}, \gamma$ es el exponente de pérdidas y $S_{\sigma}$ es el término de shadowing, el cual modela la dispersión de los valores de las pérdidas medidos respecto del valor modelado a la distancia considerada. Este término habitualmente se caracteriza estadísticamente por medio de una variable aleatoria de media 0 y desviación típica $\sigma$.

La caracterización a pequeña escala se ha realizado por medio del modelado del comportamiento dispersivo temporal del canal de propagación debido a los efectos de la propagación multicamino en el canal a la frecuencia de UWB. El valor de la dispersión o ensanchamiento temporal que sufre una señal recibida está relacionado con la máxima tasa de transferencia alcanzable en el canal, por lo tanto, es un parámetro de diseño de gran importancia. En el presente capítulo se han empleado dos métricas para cuantificar el ensanchamiento temporal de la señal: el retardo medio $\tau_{m}$ (mean excess delay) y el valor rms (root mean square) del ensanchamiento $\tau_{r m s}$ (rms delay spread), calculados de la siguiente forma (ver Apéndice A, sección A.2.1.2),

$$
\begin{gathered}
\tau_{m}=\frac{\sum_{k=1}^{L} P D P\left(\tau_{k}\right) \cdot \tau_{k}}{\sum_{k=1}^{L} P D P\left(\tau_{k}\right)} \\
\tau_{r m s}=\sqrt{\frac{\sum_{k=1}^{L} P D P\left(\tau_{k}\right) \cdot \tau_{k}^{2}}{\sum_{k=1}^{L} P D P\left(\tau_{k}\right)}-\left(\frac{\sum_{k=1}^{L} P D P\left(\tau_{k}\right) \cdot \tau_{k}}{\sum_{k=1}^{L} P D P\left(\tau_{k}\right)}\right)^{2}}
\end{gathered}
$$

donde $L$ es el número total de componentes multicamino consideradas para el cálculo. 


\subsection{Influencia del entorno de medidas en el canal de propagación off- body UWB}

La caracterización de la influencia que el entorno donde se realizan las medidas ejerce sobre las características del canal de propagación estático off-body en la banda de UWB se ha realizado a partir de las medidas de la respuesta en frecuencia del canal radio llevadas a cabo en dos entornos distintos: laboratorio TSC5 y laboratorio del SiCoMo. En cada uno de ellos se ha obtenido en primer lugar, la respuesta en frecuencia del canal de propagación entre una antena transmisora Tx fija situada a $1.4 \mathrm{~m}$ sobre el suelo y una receptora $\mathrm{Rx}$ a la misma altura ubicada a tres distancias de separación ( $1 \mathrm{~m}, 2 \mathrm{~m}$ y $3 \mathrm{~m})$, colocada sobre un soporte de madera, y en segundo lugar, se ha obtenido la respuesta en frecuencia del canal off-body entre la antena transmisora fija y una antena receptora colocada sobre la superficie del cuerpo de un sujeto a la misma altura y distancias de separación que el caso anterior. Con el fin de reducir los efectos sobre la antena causados por la interacción en campo cercano con el cuerpo, se ha empleado un elemento aislante de $10 \mathrm{~mm}$ entre la antena Rx y la superficie del mismo. En la Figura 3.7 se muestra el montaje realizado para las medidas del canal sin la influencia de un sujeto.

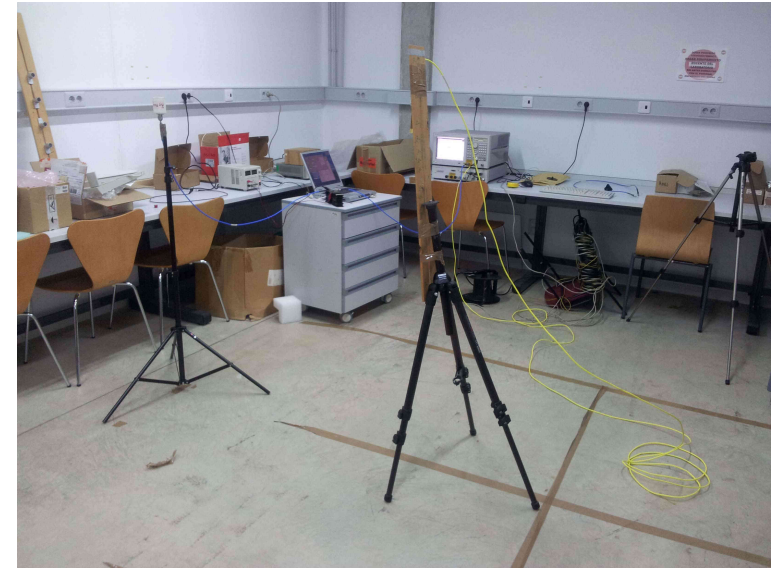

(a)

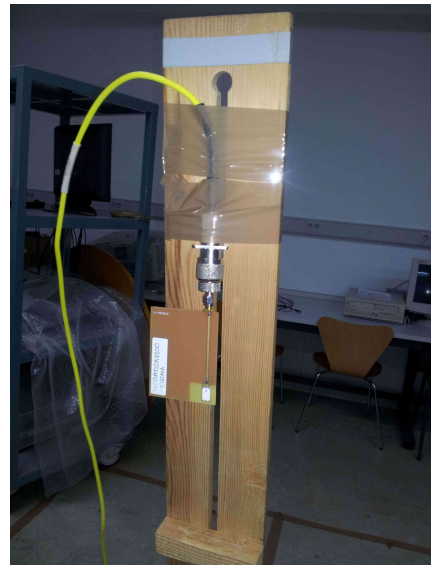

(b)

Figura 3.7: Montaje para medidas del canal sin presencia de un sujeto. (a) escenario general de medidas, (b) detalle de la antena receptora.

Las respuestas en frecuencia correspondientes a las diez realizaciones del canal radio medidas por medio del VNA han sido convertidas al dominio del tiempo para obtener la respuesta al impulso $h(\tau)$ usando la expresión (3.1). A partir de estas, se han calculado los perfiles de potencia-retardo por medio de la expresión (3.2). En la Figura 3.8 se muestran los PDPs correspondientes a las medidas realizadas a las tres distancias consideradas en los dos entornos indoor para el caso de las medidas sin y con sujetos. 


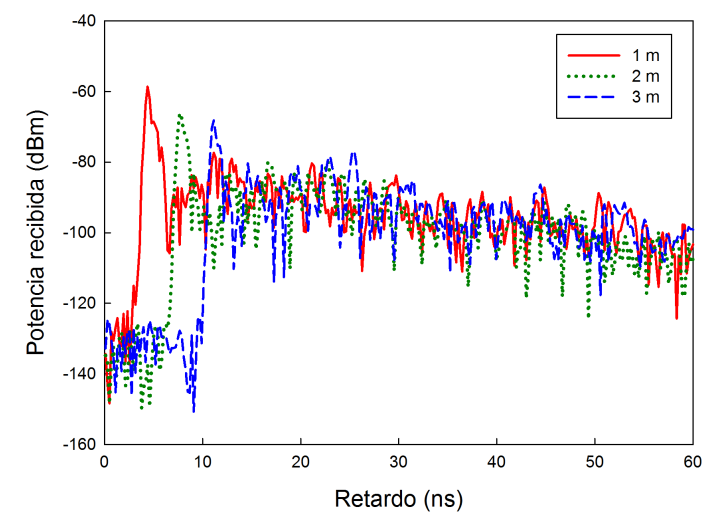

(a)

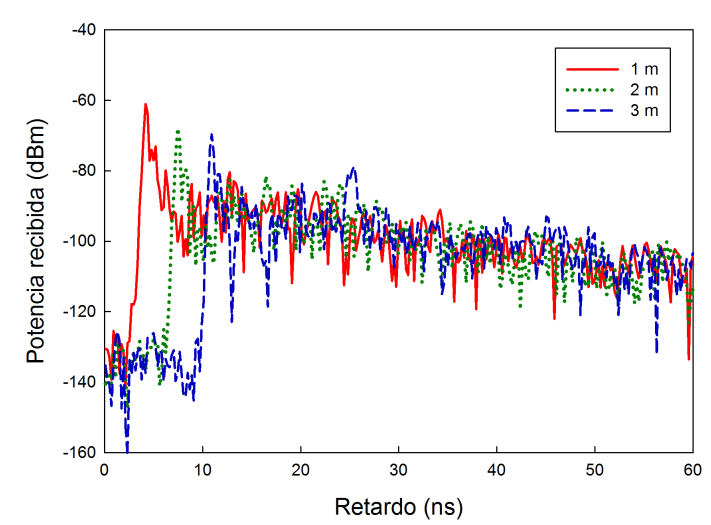

(c)

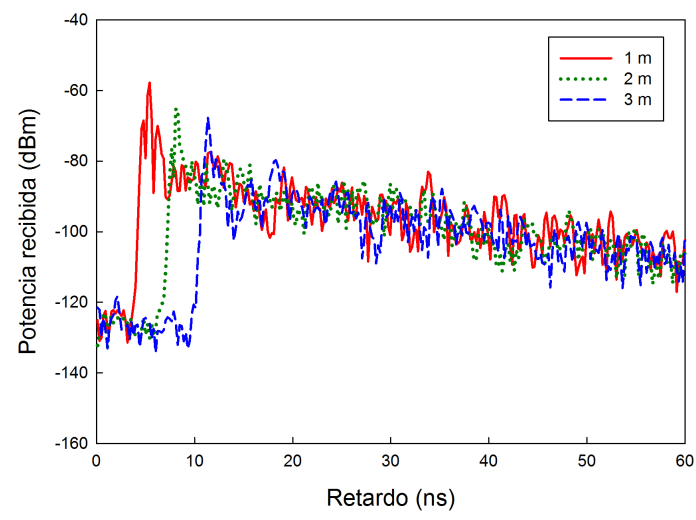

(b)

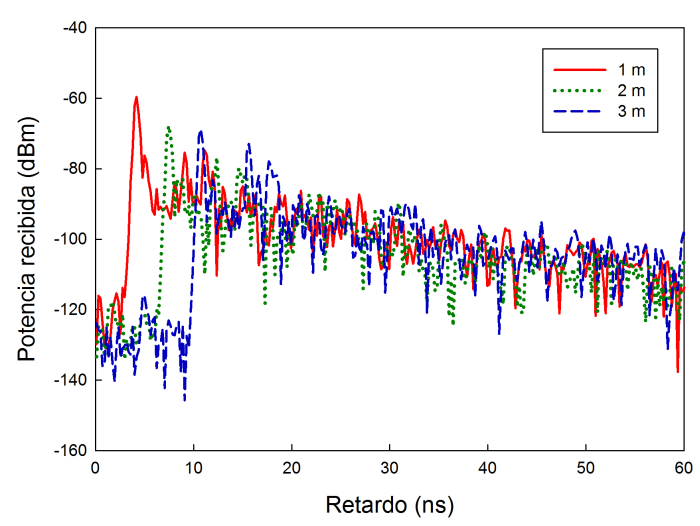

(d)

Figura 3.8: PDPs análisis de influencia del entorno de medidas. (a) Sin sujeto en laboratorio TSC5, (b) sin sujeto en laboratorio del SiCoMo, (c) con sujeto en laboratorio TSC5, (d) con sujeto en laboratorio del SiCoMo.

En el caso de las medidas sin sujetos, debido a las mayores dimensiones del laboratorio TSC5, se observa que considerando un nivel de potencia recibida umbral fija, existen componentes multicamino con potencias superiores a ese nivel a mayores retardos que para el caso del laboratorio del SiCoMo. Considerando un nivel mínimo de $-90 \mathrm{dBm}$ en el caso del TSC5, se han detectado componentes con un nivel de potencia por encima de ese umbral hasta $44 \mathrm{~ns}$ a $3 \mathrm{~m}$, mientras que para el caso del laboratorio del SiCoMo el retardo máximo es $35 \mathrm{~ns}$ a la misma distancia.

En el caso de las medidas sobre el cuerpo, debido al efecto de bloqueo o shadowing que este produce sobre las componentes multicamino, se observa que la potencia de las componentes recibidas en ambos entornos, a los mismos retardos, es similar ( $25 \mathrm{~ns}$ a $3 \mathrm{~m}$ para las medidas en el TSC5 frente a $21 \mathrm{~ns}$ en el laboratorio del SiCoMo a la misma distancia). Este efecto se va a cuantificar en los siguientes apartados. 


\subsubsection{Pérdidas de propagación y shadowing}

A partir de los perfiles de potencia-retardo calculados para cada uno de los entornos de medida y considerando el caso de propagación sin influencia del cuerpo y bajo la influencia del mismo, se han calculado las pérdidas de camino para cada caso usando la expresión (3.3). Además, se ha realizado un ajuste lineal sobre los valores de pérdidas con el fin de obtener los parámetros del modelo descrito por medio de la expresión (3.4). En la Tabla 3.4 se muestran los valores correspondientes a los parámetros del modelo de pérdidas para el caso de propagación sin influencia del cuerpo en los dos entornos.

\begin{tabular}{|c|c|c|c|}
\hline Entorno & $\boldsymbol{P \boldsymbol { L } _ { \boldsymbol { d } \boldsymbol { B } } ( \boldsymbol { d } _ { 0 } )}$ & $\boldsymbol{\gamma}$ & $\boldsymbol{\sigma}_{\boldsymbol{s}}(\boldsymbol{d B})$ \\
\hline TSC5 & 55.2 & 1.50 & 0.61 \\
\hline SiCoMo & 52.5 & 2.04 & 0.78 \\
\hline
\end{tabular}

Tabla 3.4: Valores de ajuste al modelo de pérdidas para medidas sin sujetos.

Valores para el exponente de pérdidas $\gamma$ entorno a 2 han sido reportados en estudios anteriores como en [Ghassemzadeh et al., 2003] para UWB en entornos indoor y propagación LOS.

Para la evaluación de la influencia del entorno de medidas en el canal off-body, considerando la antena Rx sobre el cuerpo, se ha seleccionado la posición 2 de colocación sobre la parte derecha de la cintura del sujeto cerca de la cadera. Este punto se ha seleccionado debido a que representa un posible lugar de colocación de un dispositivo concentrador/coordinador con mayor capacidad de proceso que un nodo normal, desde el cual se establezcan los enlaces hacia un punto de acceso (AP) externo. En la Tabla 3.5 se listan los valores para el modelo de pérdidas en el caso de la antena sobre el cuerpo. Se observa que en ambos casos el exponente de pérdidas obtenido es inferior a 2 (condiciones de propagación en espacio libre). Este valor ha sido obtenido por otros autores en otros estudios como [Abassi, 2012], [Khan et al., 2011], [Khan et al., 2013].

\begin{tabular}{|c|c|c|c|}
\hline Entorno & $\boldsymbol{P \boldsymbol { L } _ { \boldsymbol { d } \boldsymbol { B } } ( \boldsymbol { d } _ { 0 } )}$ & $\boldsymbol{\gamma}$ & $\boldsymbol{\sigma}_{\boldsymbol{S}}(\boldsymbol{d B})$ \\
\hline TSC5 & 57.4 & 1.53 & 0.47 \\
\hline SiCoMo & 55.8 & 1.83 & 0.52 \\
\hline
\end{tabular}

Tabla 3.5: Valores de ajuste al modelo de pérdidas para medidas con sujetos.

Tal y como se observa en la Figura 3.9 la diferencia en el valor de las pérdidas de propagación para el canal off-body entre una antena colocada sobre el cuerpo de un sujeto y una antena externa, respecto al valor medido sin la influencia del cuerpo, está en torno a los $2 \mathrm{~dB}$ en los dos entornos indoor considerados. 


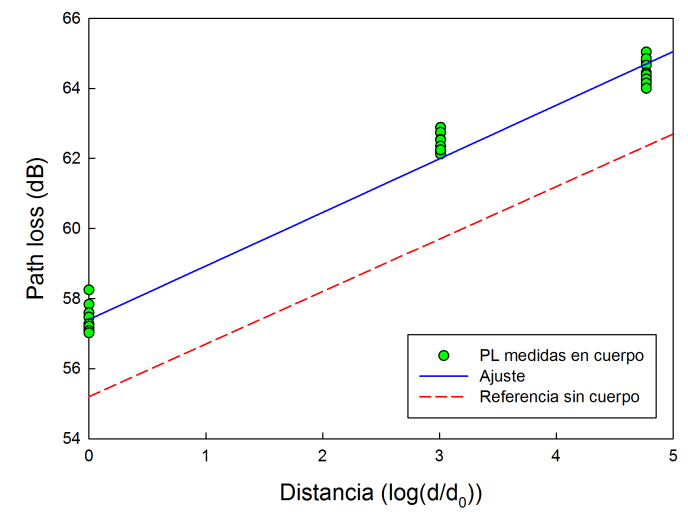

(a)

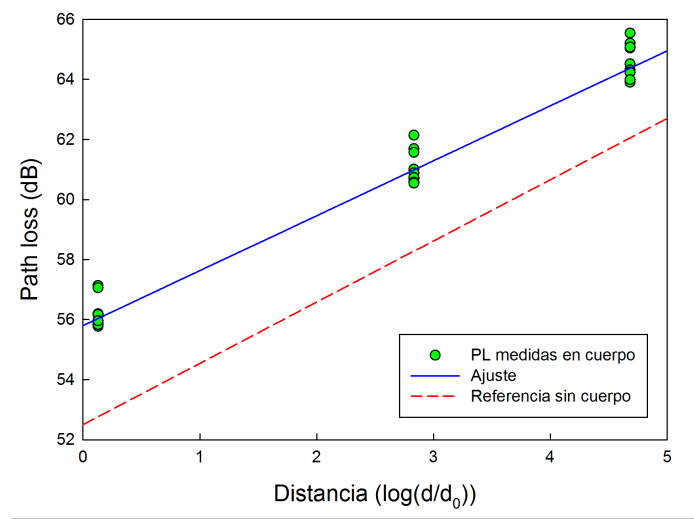

(b)

Figura 3.9: Variación de las pérdidas de camino respecto del entorno de medidas en medidas con y sin cuerpo. (a) TSC5, (b) SiCoMo.

Otro efecto observado es la reducción en la desviación típica del shadowing para el caso de la antena en el cuerpo de un sujeto respecto al correspondiente sin considerar sujeto. Esto se debe a que al estar considerando condiciones de propagación por visión directa (LOS) y debido al efecto de bloqueo por parte del cuerpo, el número de componentes multicamino recibidas a retardos mayores a la distancia entre Tx y Rx se reduce respecto al número recibido sin considerar la antena Rx sobre el cuerpo. En el caso de que la propagación hubiera sido sin visión directa (NLOS) el efecto habría sido el contrario, debido a que el mecanismo de propagación principal entre Tx y Rx es el multicamino, ya que a frecuencias de UWB una señal no se propaga a través del cuerpo. En [Kahn, 2011] se han obtenido variaciones en el exponente de pérdidas entre LOS y NLOS del $32.17 \%$ con un aumento en el valor de pérdidas para una distancia de referencia de $1 \mathrm{~m}$ en torno a $7 \mathrm{~dB}$.

Respecto al exponente de pérdidas se observa que tanto para el caso del canal radio considerando la antena sin sujeto y con sujeto en el laboratorio TSC5 el valor es muy similar, mientras que para el laboratorio del SiCoMo se observa una disminución el valor del exponente de pérdidas para el caso de la antena sobre el sujeto respecto a sin este. Esto puede estar debido a que, por el efecto de bloqueo del cuerpo, el número de componentes recibidas con un nivel de potencia similar a la componente por visión directa es menor, esto hace que a la hora calcular las pérdidas, exista poca variación en la potencia de la componente principal a las distancias consideradas y al realizar el modelado, el exponente de pérdidas sea algo menor al obtenido sin la influencia del sujeto. 


\subsubsection{Dispersión temporal del canal radio}

El efecto de ensanchamiento temporal de una señal recibida debido al efecto multicamino en el canal de propagación se puede modelar en función del ensanchamiento de retardo medio (mean excess delay) $\tau_{m}$ calculado usando la expresión (3.5) y el valor rms del ensanchamiento (rms delay spread) $\tau_{r m s}$ calculado por medio de la expresión (3.6). Para la obtención de estos parámetros a partir de los perfiles de potencia-retardo, se han aplicado dos valores de umbral bajo la componente más significativa: $20 \mathrm{~dB}$ y $30 \mathrm{~dB}$, de manera que las componentes cuyo nivel de potencia se encuentran por bajo de este umbral son descartadas. Por lo tanto, la variación de este umbral tendrá una repercusión en los valores medidos para el ensanchamiento del canal debido a que variará el número efectivo de componentes que se tendrán en cuenta para el cálculo.

En las Tablas 3.6 y 3.7 se muestran los valores medios, sin y con la influencia del cuerpo en las medidas $\left(\mu_{f s} \mathrm{y} \mu_{s u}\right)$, y desviación típica $\left(\sigma_{f s} \mathrm{y} \sigma_{s u}\right)$ para los parámetros de dispersión $\tau_{r m s}$ y $\tau_{m}$ a los dos umbrales considerados para las medidas.

\begin{tabular}{|c|c|c|c|c|c|c|c|c|c|}
\hline \multicolumn{10}{|c|}{$\tau_{r m s}(n \boldsymbol{s})$} \\
\hline \multirow{2}{*}{$\begin{array}{c}\text { Umbral } \\
\text { (dB) }\end{array}$} & \multirow{2}{*}{$\begin{array}{l}\text { Distancia } \\
\text { (m) }\end{array}$} & \multicolumn{4}{|c|}{ TSC5 } & \multicolumn{4}{|c|}{$\begin{array}{l}\text { SiCoMo } \\
\end{array}$} \\
\hline & & $\mu_{f s}$ & $\mu_{s u}$ & $\sigma_{f s}$ & $\sigma_{s u}$ & $\mu_{f s}$ & $\mu_{s u}$ & $\sigma_{f s}$ & $\sigma_{s u}$ \\
\hline \multirow[t]{3}{*}{$20 \mathrm{~dB}$} & $1 \mathrm{~m}$ & 0.84 & 0.73 & 0.12 & 0.14 & 0.86 & 1.92 & 0.45 & 0.05 \\
\hline & $2 \mathrm{~m}$ & 4.68 & 4.03 & 0.15 & 0.08 & 2.13 & 2.55 & 1.09 & 0.06 \\
\hline & $3 \mathrm{~m}$ & 6.49 & 5.68 & 0.09 & 0.24 & 4.56 & 2.86 & 0.93 & 0.19 \\
\hline \multirow[t]{3}{*}{$30 \mathrm{~dB}$} & $1 \mathrm{~m}$ & 3.74 & 2.66 & 0.08 & 0.07 & 2.98 & 2.46 & 0.33 & 0.10 \\
\hline & $2 \mathrm{~m}$ & 6.68 & 5.74 & 0.09 & 0.12 & 6.13 & 3.64 & 0.49 & 0.04 \\
\hline & $3 \mathrm{~m}$ & 8.89 & 7.61 & 0.02 & 0.15 & 6.68 & 3.93 & 0.61 & 0.26 \\
\hline
\end{tabular}

Tabla 3.6: rms Delay Spread para medidas con y sin sujeto en laboratorios TSC5 y SiCoMo.

\begin{tabular}{|c|c|c|c|c|c|c|c|c|c|}
\hline \multicolumn{10}{|c|}{$\tau_{m}(n s)$} \\
\hline \multirow{2}{*}{$\begin{array}{l}\text { Umbral } \\
\text { (dB) }\end{array}$} & \multirow{2}{*}{$\begin{array}{l}\text { Distancia } \\
\text { (m) }\end{array}$} & \multicolumn{4}{|c|}{ TSC5 } & \multicolumn{4}{|c|}{ SiCoMo } \\
\hline & & $\mu_{f s}$ & $\mu_{s u}$ & $\sigma_{f s}$ & $\sigma_{s u}$ & $\mu_{f s}$ & $\mu_{s u}$ & $\sigma_{f s}$ & $\sigma_{s u}$ \\
\hline \multirow{3}{*}{$20 \mathrm{~dB}$} & $1 \mathrm{~m}$ & 0.38 & 0.31 & 0.03 & 0.02 & 0.41 & 1.25 & 0.18 & 0.07 \\
\hline & $2 \mathrm{~m}$ & 2.25 & 1.82 & 0.08 & 0.08 & 1.07 & 2.69 & 0.75 & 0.08 \\
\hline & $3 \mathrm{~m}$ & 5.06 & 3.57 & 0.04 & 0.43 & 2.73 & 2.74 & 0.54 & 0.12 \\
\hline \multirow{3}{*}{$30 \mathrm{~dB}$} & $1 \mathrm{~m}$ & 1.27 & 0.85 & 0.02 & 0.02 & 1.09 & 1.56 & 0.08 & 0.11 \\
\hline & $2 \mathrm{~m}$ & 3.55 & 3.06 & 0.04 & 0.07 & 3.35 & 3.27 & 0.67 & 0.06 \\
\hline & $3 \mathrm{~m}$ & 6.86 & 5.19 & 0.03 & 0.42 & 4.22 & 3.30 & 0.54 & 0.15 \\
\hline
\end{tabular}

Tabla 3.7: Mean Excess Delay para medidas con y sin sujeto en laboratorios TSC5 y SiCoMo.

Respecto a la variación en la dispersión temporal del canal radio, en general se observa un efecto de reducción en el valor tanto de $\tau_{r m s}$ como de $\tau_{m}$ para el caso de las medidas en el SiCoMo respecto al TSC5. Sin la influencia del cuerpo en las medidas, esta 
reducción va desde un $29 \%$ a 3 m para un umbral de $20 \mathrm{~dB}$ a un $24 \%$ a $3 \mathrm{~m}$ en el caso de 30 dB. En el caso de la antena sobre el cuerpo esta reducción va desde un $49 \%$ para $3 \mathrm{~m}$ a $20 \mathrm{~dB}$ hasta un $48 \%$ a $3 \mathrm{~m}$ para $30 \mathrm{~dB}$. Esto se puede justificar por la diferencia de tamaños entre ambos entornos. En el caso del laboratorio del SiCoMo, al ser menor que el TSC5 las componentes multicamino llegan al receptor a menor nivel de retardo y se atenúan más rápido (a partir de $35 \mathrm{~ns}$ a $3 \mathrm{~m}$ las componentes presentan un nivel de potencia inferior a $-90 \mathrm{~dB}$ frente a los 44 ns en el TSC5) por lo que, en general el efecto de ensanchamiento temporal producido en la señal recibida es menor.

En la Figura 3.10 se representa la dependencia del incremento en la dispersión temporal en el canal respecto a la variación en las pérdidas de propagación en cada uno de los entornos para los casos sin sujeto y con sujeto.

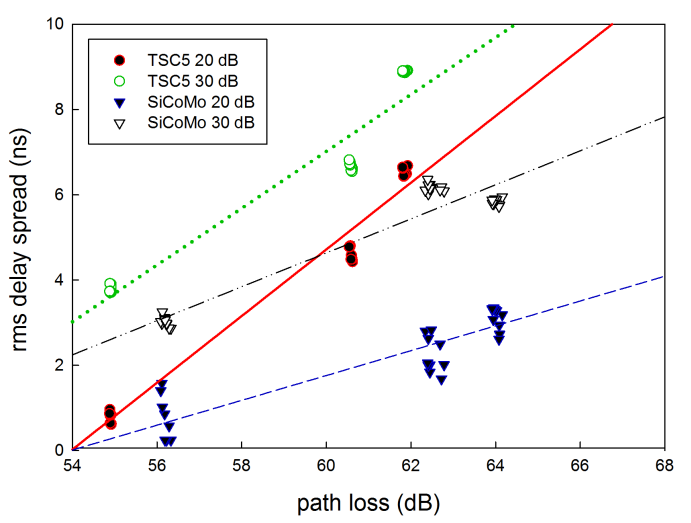

(a)

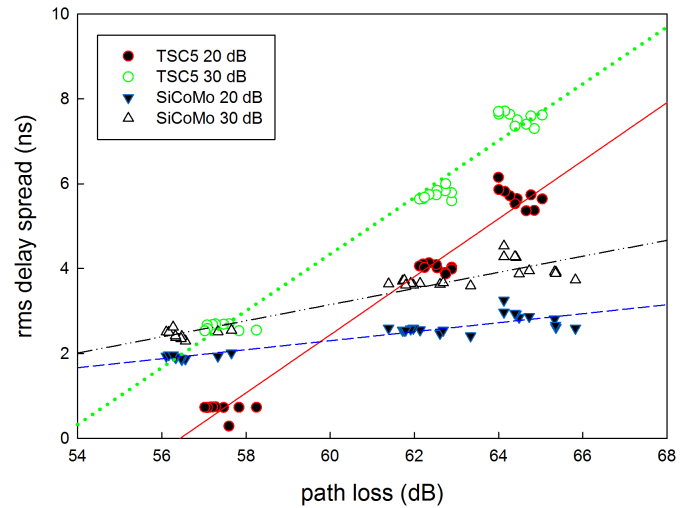

(b)

Figura 3.10: Variación del ensanchamiento de retardo del canal por entorno de medidas. (a) Sin sujeto, (b) con antena en sujeto.

A partir de la Figura 3.10 podemos observar que el ensanchamiento de retardo del canal off-body está relacionado con el aumento de las pérdidas de propagación. En referencia a la dispersión de los valores para $\tau_{r m s}$ respecto a las perdidas de propagación, se observa una alta variabilidad con el umbral escogido en el caso de las medidas sin sujetos. El valor de dispersión promedio obtenido en el laboratorio del SiCoMo para $20 \mathrm{~dB}$ es superior al obtenido para las medidas en el laboratorio TSC5. Esto puede deberse a que, por las mayores dimensiones de este, las componentes llegan más atenuadas y a ese nivel de umbral se estén rechazando componentes que deberían tenerse en cuenta para el cálculo. Para un umbral de $30 \mathrm{~dB}$ se observa que la dispersión promedio del valor de $\tau_{r m s}$ en el caso del SiCoMo para el canal sin sujeto es un $28 \%$ inferior a la dispersión medida en el TSC5. Por otro lado, considerando las medidas con la antena sobre los sujetos, se observa que la dispersión es inferior al caso del canal sin sujeto y que, considerando un valor de umbral de $30 \mathrm{~dB}$, se 
observa una reducción promedio similar en ambos entornos del 41\%. Esta reducción se debe al efecto de bloqueo de componentes multicamino causado por el cuerpo.

\subsection{Influencia de la posición de colocación de una antena sobre el cuerpo en el canal off-body UWB}

Debido a las características morfológicas y dieléctricas del cuerpo humano, la posición de colocación de una antena sobre el cuerpo de un sujeto puede afectar a la propagación en los enlaces off-body entre esta y un punto de acceso situado en las inmediaciones.

\subsubsection{Esquema de medidas}

Las medidas se han realizado considerado condiciones de propagación LOS en un entorno indoor para el cual se ha elegido el interior del laboratorio del SiCoMo. Las medidas se han llevado a cabo sobre tres sujetos voluntarios cuyas características se describen en la Tabla 3.3. Los sujetos se han colocado en posición de pie a tres distancias de separación (1 m, $2 \mathrm{~m}$ y $3 \mathrm{~m}$ ) de la antena Tx colocada a una altura de $1.4 \mathrm{~m}$ sobre el suelo. Durante el tiempo de medidas estos han permanecido estáticos con los brazos paralelos al cuerpo con el fin de reducir las variaciones en el canal debidas al movimiento. Se han considerado las cinco posiciones de colocación de la antena receptora Johanson Tec. 3100AT51A7200 identificadas anteriormente sobre el cuerpo de los sujetos. En la Figura 3.11 se indica tanto las posiciones de colocación de la antena Rx sobre el cuerpo como la ubicación de los sujetos.

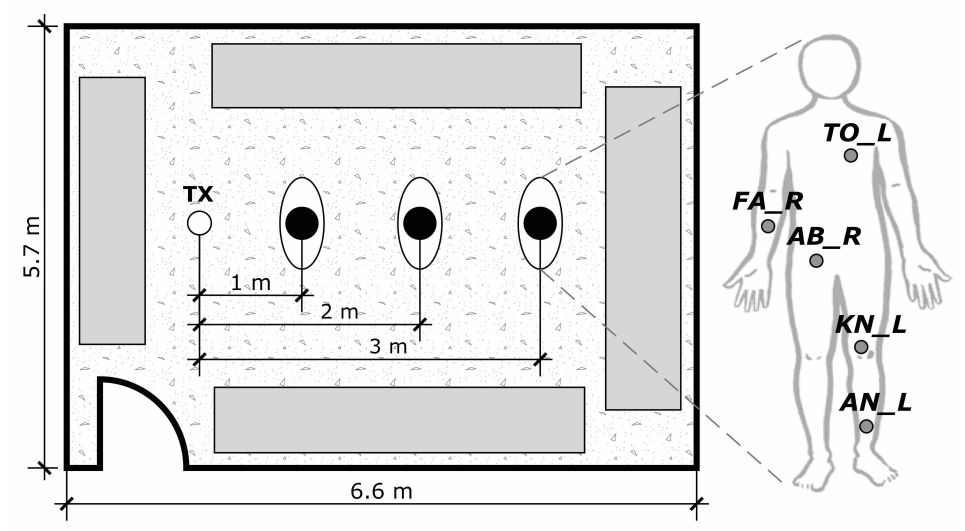

Figura 3.11: Entorno de medidas y posiciones de colocación de la antena Rx sobre el cuerpo.

Las medidas del canal radio se han realizado empleando el método medidas en frecuencia descrito en la sección 3.2, con el analizador de redes vectorial Agilent ENA E5071B como dispositivo de generación de la señal de barrido y medida del parámetro de dispersión $S_{21}$ equivalente a la respuesta en frecuencia del canal. 


\subsubsection{Pérdidas de propagación y shadowing}

El modelado de las pérdidas de propagación en cada uno de los enlaces considerados entre la antena Tx y los puntos de colocación de la antena Rx sobre el cuerpo de los sujetos a las tres distancias de separación consideradas, se ha realizado mediante el análisis de los perfiles de potencia-retardo obtenidos a partir de las medidas de las respuestas en frecuencia del canal radio para cada combinación de sujeto, enlace y distancia por medio de las expresiones (3.1) y (3.2). Las pérdidas de propagación obtenidas empleando la expresión (3.3) se han ajustado al modelo definido por la expresión (3.4) por medio de un ajuste lineal de los datos. En la Tabla 3.8 se muestran los valores del modelo para cada enlace considerado en cada uno de los sujetos.

\begin{tabular}{|c|c|c|c|}
\hline \multirow{2}{*}{ Enlace } & \multicolumn{3}{|c|}{$\boldsymbol{P L}_{d \boldsymbol{B}}\left(\boldsymbol{d}_{0}\right)(\boldsymbol{d B}) / \boldsymbol{\gamma} / \boldsymbol{\sigma}_{S}(\boldsymbol{d B})$} \\
\cline { 2 - 4 } & Sujeto 1 & Sujeto 2 & Sujeto 3 \\
\hline TO_L & $55.1 / 1.73 / 0.8$ & $52.5 / 2.21 / 1.1$ & $56.4 / 1.82 / 0.6$ \\
\hline AB_R & $55.8 / 1.83 / 0.5$ & $46.2 / 2.39 / 0.5$ & $57.3 / 2.23 / 0.8$ \\
\hline FA_R & $56.6 / 1.62 / 0.7$ & $50.7 / 2.67 / 1.2$ & $61.4 / 1.28 / 1.3$ \\
\hline KN_L & $49.8 / 1.95 / 0.3$ & $46.9 / 2.75 / 0.3$ & $52.2 / 1.38 / 0.8$ \\
\hline AN_L & $48.8 / 2.56 / 1.1$ & $48.3 / 3.90 / 1.6$ & $50.9 / 2.77 / 1.1$ \\
\hline Todos & $54.3 / 1.59 / 2.5$ & $49.2 / 2.75 / 3.1$ & $56.7 / 1.55 / 3.4$ \\
\hline
\end{tabular}

Tabla 3.8: Parámetros del modelo de pérdidas para cada enlace off-body Tx-Rx.

Al igual que se ha reportado en [Abbasi et al., 2012], para un rango de distancias entre 0.1-1 m entre un sujeto y un punto de acceso a $1 \mathrm{~m}$ del suelo en la banda de 3-10 GHz considerando 109 puntos de colocación de una antena sobre el cuerpo, los valores de $P L_{d B}\left(d_{0}\right)$ a una distancia de $1 \mathrm{~m}$ para los enlaces correspondientes a los puntos de colocación en la parte inferior de los sujetos (KN_L y AN_L), son menores a los del resto de enlaces. Esto puede deberse a que las componentes multicamino recibidas por reflexiones en el suelo pueden incrementar el nivel de señal recibido y por lo tanto reducirse el nivel de pérdidas a esta distancia. Este efecto no se observa en los valores para $P L_{d B}\left(d_{0}\right)$ reportados en [Khan et al., 2011], considerando medidas a 6 distancias (1-6 m) entre un sujeto y punto de acceso a $1 \mathrm{~m}$ sobre el suelo en la banda de 5.9-7.25 GHz considerando 5 puntos de colocación de una antena sobre el cuerpo. En este estudio, los puntos de colocación de la antena por encima de la cintura son los que menores valores de pérdidas presentan. Además, los valores reportados en este estudio, en comparación con los mostrados en la Tabla 3.8, son superiores al $20 \%$.

Respecto a los valores obtenidos para el exponente de pérdidas $\gamma$, los obtenidos para los tres sujetos considerados son mayores tanto a los reportados en [Abbasi et al., 2012] (0.26 para el área de las piernas hasta 1.89 para el tronco), como a los reportados en [Khan et al., 
2011]. En este último estudio, el exponente de pérdidas obtenido para la posición de colocación de una antena en el tobillo, en condiciones de propagación LOS, es de 1.2 siendo las posiciones con menor exponente las correspondientes a la cintura $(\gamma=0.84)$ y muñeca $(\gamma=0.96)$. En las medidas realizadas sobre los sujetos 1 a 3, aunque posiciones de colocación similares (AB_R y FA_R) presentan valores para el exponente de pérdidas menores a los obtenidos para el resto de posiciones, este hecho no se cumple de manera igual en los tres sujetos. En los resultados mostrados en la Tabla 3.8 se puede ver que, el valor obtenido para el exponente de pérdidas $\gamma$ en el punto de colocación AN_L correspondiente a la parte inferior de la espinilla es el mayor en comparación con el resto para los tres sujetos. Esto puede deberse a que esta posición es la que presenta peor nivel de alineamiento entre las antenas Tx y Rx por lo que, aunque a la distancia de referencia el valor de $P L_{d B}\left(d_{0}\right)$ sea bajo, el nivel de pérdidas con la distancia sea mayor que para el resto de enlaces. Esto es coherente con el hecho de que las posiciones ubicadas por encima de la cintura, por lo general, presentan valores del exponente de pérdidas más bajos.

En la Tabla 3.9 se muestra la variación en el número medio de componentes multicamino recibidas ( $\bar{L}$ ) para cada enlace y sujeto considerando (S1, S2 y S3). Únicamente se han considerado para el cálculo aquellas que se encuentran por encima de un umbral de $-30 \mathrm{~dB}$ bajo la componente más significativa.

\begin{tabular}{|c|c|c|c|}
\hline \multirow{2}{*}{ Enlace } & \multicolumn{3}{|c|}{$\bar{L}_{30 d B}(S 1 / S 2 / S 3)$} \\
\hline & $1 \mathrm{~m}$ & $2 \mathrm{~m}$ & $3 \mathrm{~m}$ \\
\hline TO_L & $19 / 20 / 37$ & $30 / 32 / 43$ & $38 / 50 / 61$ \\
\hline AB $R$ & $17 / 15 / 21$ & $32 / 24 / 27$ & $35 / 27 / 29$ \\
\hline FA_R & $22 / 16 / 21$ & $31 / 30 / 37$ & $38 / 32 / 38$ \\
\hline KN_L & $17 / 21 / 20$ & $23 / 30 / 27$ & $32 / 36 / 26$ \\
\hline AN_L & $16 / 20 / 23$ & $27 / 30 / 27$ & $30 / 44 / 27$ \\
\hline
\end{tabular}

Tabla 3.9: Número medio de componentes multicamino recibidas para cada punto de colocación de Rx.

Promediando los valores para las pérdidas de propagación para todos los enlaces considerados para cada una de las distancias en cada sujeto, y comparando estos valores con respecto de los medidos y reportados en la Tabla 3.4 considerando propagación sin influencia del cuerpo en el SiCoMo, en la Figura 3.12 se muestra la variación media para cada enlace respecto de las pérdidas de referencia sin cuerpo. 


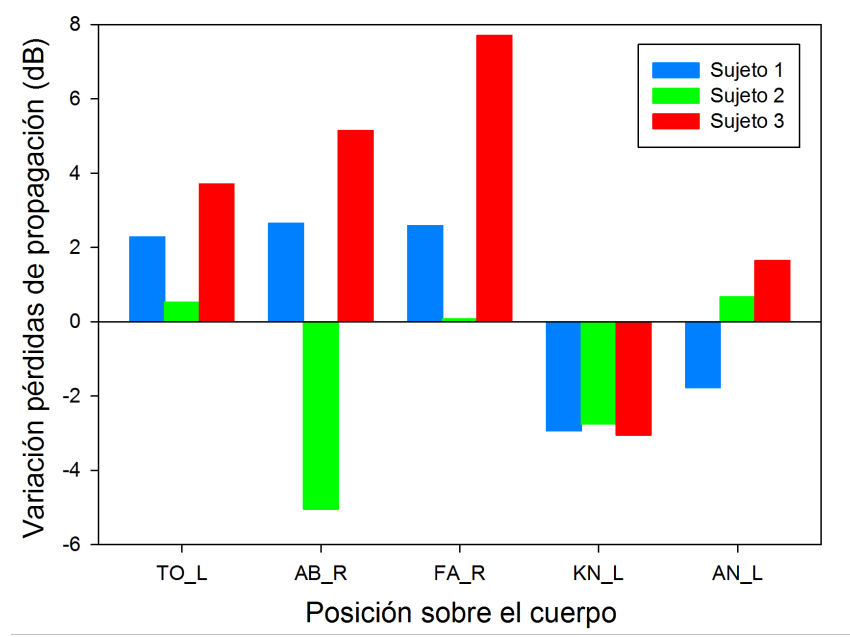

Figura 3.12: Valor medio de pérdidas de propagación por cada enlace en el cuerpo respecto a los valores de referencia sin cuerpo.

A partir de los valores de la Figura 3.12 se puede observar que las posiciones de colocación de la antena Rx sobre el cuerpo de un sujeto que mayor variabilidad presentan respecto al valor de las pérdidas de propagación, en comparación con los valores sin la influencia del cuerpo, son la correspondiente al punto de colocación en la parte derecha del abdomen ( $\left.A B \_R\right)$ con una variación de entre $2.7 \mathrm{~dB}$ y $5.3 \mathrm{~dB}$ entorno a un valor medio de pérdidas de $58.4 \mathrm{~dB}$ y la posición de colocación en el antebrazo derecho (FA_R) con una variación de entre $0.1 \mathrm{~dB}$ y $7.9 \mathrm{~dB}$ en torno a un valor de pérdidas de $58.7 \mathrm{~dB}$. Por el contrario, las posiciones con menor variabilidad son las correspondientes a la parte superior derecha del torso (TO_L) con valores entre $0.5 \mathrm{~dB}$ y $3.7 \mathrm{~dB}$ entorno a un valor medio de pérdidas de $58.2 \mathrm{~dB}$ y la espinilla derecha (AN_L) con diferencias en las pérdidas entre $0.7 \mathrm{~dB}$ y $1.7 \mathrm{~dB}$ entorno a un valor de $60.1 \mathrm{~dB}$. En la Figura 3.12 los valores de variación por bajo de 0 indican valores de perdidas medidos inferiores a los obtenidos en el caso de considerar el canal sin la influencia de un sujeto. En general se observa que la morfología y la orientación de las antenas en el punto de colocación sobre el cuerpo, son factores que pueden influir notablemente en el valor medido para las pérdidas de propagación en el canal off-body.

\subsubsection{Dispersión temporal del canal radio}

La caracterización de la dispersión temporal del canal radio debido a la propagación multicamino se ha realizado a partir del análisis del parámetro de dispersión de retardo rms delay spread, obtenido a partir del perfil de potencia-retardo por medio de la expresión (3.6). Con el fin de eliminar las contribuciones debidas a componentes cercanas al umbral de ruido del sistema, se ha empleado un valor de umbral de $30 \mathrm{~dB}$ bajo el nivel de potencia 
recibido para la componente más significativa. Adicionalmente, los valores obtenidos han sido comparados con los obtenidos a través de las medidas realizadas sin la influencia del cuerpo en el interior del SiCoMo y recogidos en la Tabla 3.6. Los valores para el rms delay spread $\tau_{\text {rms }}$ obtenidos para cada enlace entre Tx y cada posición de colocación de Rx sobre el cuerpo de los tres sujetos (S1, S2 y S3) a las tres distancias consideradas se muestran en la Tabla 3.10.

\begin{tabular}{|c|c|c|c|c|c|c|c|c|c|}
\hline \multirow{2}{*}{ Enlace } & \multicolumn{10}{|c|}{$\tau_{\text {rms }}(\boldsymbol{n s})$} \\
\cline { 2 - 11 } & \multicolumn{3}{|c|}{$\mathbf{1 ~ m}$} & \multicolumn{3}{|c|}{$\mathbf{~ m}$} & \multicolumn{3}{|c|}{$\mathbf{3 ~ m}$} \\
\cline { 2 - 10 } & S1 & S2 & S3 & S1 & S2 & S3 & S1 & S2 & S3 \\
\hline TO_L & 2.11 & 2.16 & 3.24 & 3.88 & 4.16 & 5.27 & 4.62 & 6.61 & 6.02 \\
\hline AB_R & 2.39 & 1.96 & 2.35 & 3.51 & 3.17 & 3.21 & 3.91 & 3.46 & 3.94 \\
\hline FA_R & 2.73 & 1.64 & 2.26 & 4.06 & 3.71 & 3.70 & 5.78 & 4.46 & 3.99 \\
\hline KN_L & 1.83 & 2.01 & 2.15 & 2.39 & 3.74 & 2.59 & 4.22 & 4.46 & 3.03 \\
\hline AN_L & 1.63 & 1.98 & 2.3 & 3.15 & 4.18 & 2.46 & 3.76 & 6.63 & 2.96 \\
\hline
\end{tabular}

Tabla 3.10: rms delay spread a $30 \mathrm{~dB}$ para las posiciones de Rx sobre el cuerpo de los sujetos a las tres distancias consideradas.

Valores medios para $\tau_{r m s}$ de $3.34 \mathrm{~ns}$ a $30 \mathrm{~dB}$ de umbral se han reportado con anterioridad en [Abbasi et al., 2012]. Comparado con los valores sin tener en cuenta la influencia del cuerpo considerando indoor y LOS, se observa un efecto de reducción relacionado con el bloqueo de componentes multicamino por partes del cuerpo. En la Figura 3.13 se muestra este efecto de reducción en el valor del rms delay spread considerando una distancia de referencia de $2 \mathrm{~m}$ para los enlaces considerados.

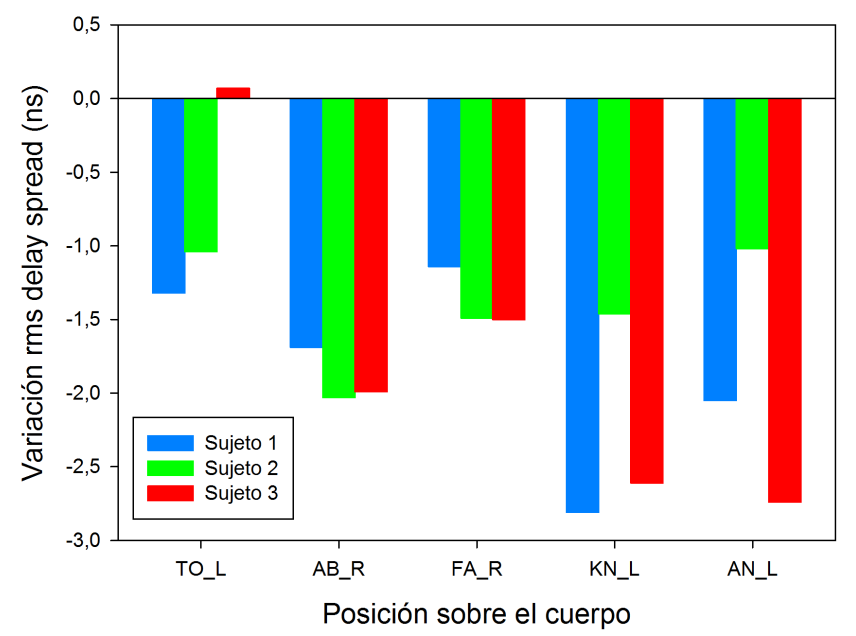

Figura 3.13: Variación media en el rms delay spread para cada posición de Rx en el cuerpo con sujetos a $2 \mathrm{~m}$ respecto al valor sin unfluencia del cuerpo.

A partir de la Figura 3.13 se puede observar que los enlaces que mayor variabilidad presentan respecto al valor de la dispersión temporal del canal radio, son los correspondientes 
a las posiciones de colocación de $\mathrm{Rx}$ AB_R (1.99 ns), KN_L (2.61 ns) y AN_L (2.05 ns). Respecto al número medio de componentes multicamino recibidas considerando cada una de las posiciones de colocación anteriores, estas presentan una reducción del 25.2\%, $29.9 \%$ y $24.3 \%$ respectivamente. Por el contrario, TO_L presenta un número de componentes multicamino recibido cercano al valor medido sin la influencia del sujeto en el SiCoMo, por lo tanto, la variación en el valor de $\tau_{r m s}$ es la menor respecto al resto de posiciones, con valores de $1.32 \mathrm{~ns}, 1.04 \mathrm{~ns}$ y $0.1 \mathrm{~ns}$.

\subsection{Influencia de la postura en el canal de propagación off-body UWB}

La posición adoptada por un sujeto en el cual se encuentra desplegada una red WBAN puede ocasionar la variación en el canal off-body entre dispositivos sobre el cuerpo y un elemento externo, a cierta distancia, que actúe como punto de acceso. Entre las posibles posturas de interés, en el presente apartado se han considerado las correspondientes a de pie y tumbado bajo condiciones estáticas en el canal y visión directa.

\subsubsection{Esquema de medidas}

El modelado del canal off-body para un conjunto de enlaces entre una antena Tx y una Rx sobre el cuerpo de un sujeto, considerando la postura adoptada por el mismo, se ha caracterizado en términos de la dispersión temporal y la variación de los parámetros del modelo de respuesta al impulso CM4 propuesto en el estándar IEEE 802.15.6 para la banda de UWB. Para ello se han realizado una serie de medidas en frecuencia del canal radio en el interior del laboratorio del SiCoMo, empleando el esquema de medidas mostrado en la Figura 3.1 con el analizador de redes configurado según los parámetros de la Tabla 3.1. Para estas medidas, se han considerado dos sujetos (Sujetos 1 y 2) cuyas características se detallan en la Tabla 3.3. En primer lugar, los sujetos se han colocado en posición de pie con los brazos paralelos al cuerpo frente a la antena transmisora situada sobre un trípode a una distancia de separación fija de $2 \mathrm{~m}$ y a $1.4 \mathrm{~m}$ de altura. En esta disposición se ha colocado la antena receptora en los cinco puntos considerados sobre la superficie del cuerpo de cada sujeto. En un segundo grupo de medidas se ha colocado a cada uno de los sujetos en posición tumbada sobre una mesa dispuesta a modo de camilla de dimensiones $1.9 \mathrm{x} 0.5 \mathrm{~m}$ cuyo punto central se encuentra a $2 \mathrm{~m}$ respecto a Tx y la antena $\mathrm{Rx}$ se ha colocado sobre los mismos puntos considerados en el caso del sujeto en posición de pie. En la Figura 3.14 se muestra el esquema general empleado para las medidas con sujetos de pie y tumbados. 


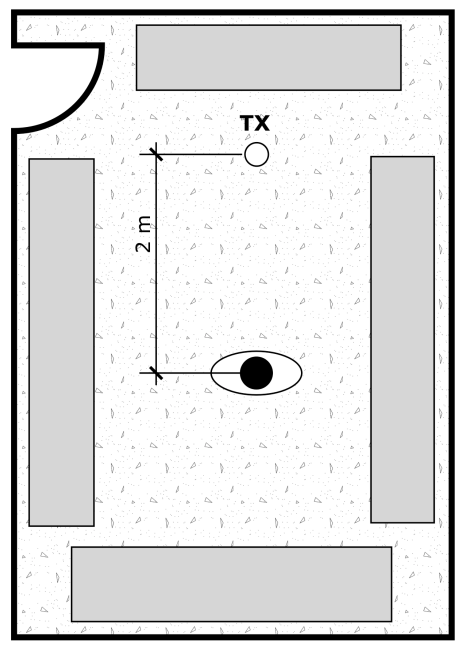

(a)

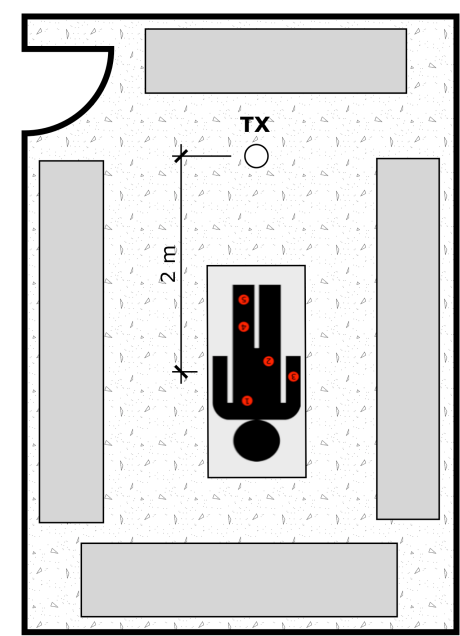

(b)

Figura 3.14: Esquema de medidas con sujetos de pie (a) y tumbados (b).

En la Figura 3.15 se muestra la disposición real de la mesa dispuesta a modo de camilla para la realización de las medidas sobre los sujetos tumbados.

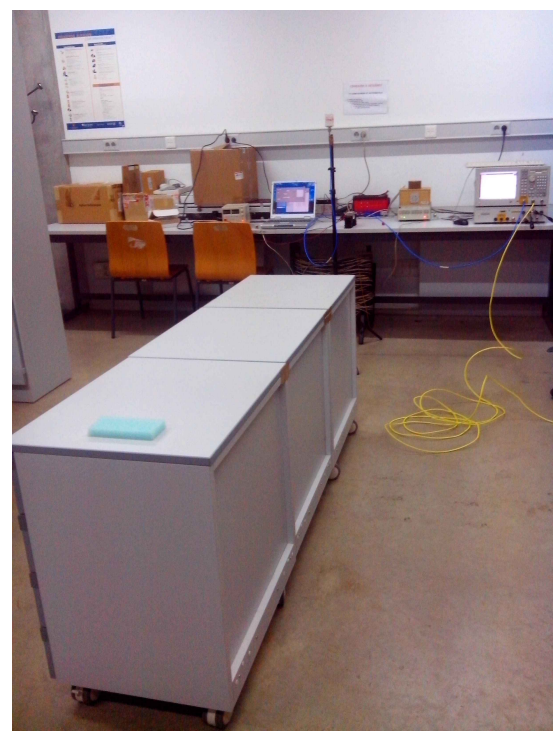

Figura 3.15: Mesa para medidas con sujetos tumbados.

Las medidas del canal se han realizado empleando el método en frecuencia descrito en el punto 3.2, con el analizador de redes vectorial Agilent ENA E5071B como dispositivo de generación de la señal de barrido y medida de la respuesta en frecuencia del canal. Para cada posición de la antena receptora se han tomado diez realizaciones de la función de transferencia del canal (parámetro de transmisión $S_{21}$ ) que posteriormente han sido transformadas en las correspondientes respuestas al impulso por medio de la transformada inversa de Fourier. A partir de las respuestas al impulso correspondientes a las medidas del canal de propagación para cada enlace considerado en cada uno de los dos sujetos dispuestos 
en posición de pie y tumbados, se ha obtenido el perfil de potencia-retardo por medio de la expresión (3.2).

\subsubsection{Dispersión temporal del canal radio}

La influencia de la postura de un sujeto en la variación de la dispersión temporal que experimenta el canal de propagación off-body entre una antena transmisora y una receptora sobre el cuerpo de un sujeto para cada posición de colocación de Rx considerada se ha caracterizado a partir del parámetro de ensanchamiento rms delay spread considerando un umbral de $-30 \mathrm{~dB}$ bajo la potencia de la contribución más significativa, con el fin de eliminar contribuciones debidas al ruido en las medidas. Los valores obtenidos para cada sujeto en cada una de las posturas se describen en la Tabla 3.11. Con el objetivo de evaluar la variación en el número de componentes multicamino recibidas en cada una de las posiciones de Rx sobre el cuerpo de cada sujeto de pie respecto del número recibido para la misma posición cuando se encuentra tumbado, en la Tabla 3.12 se muestra el número medio de componentes para cada caso.

\begin{tabular}{|c|c|c|c|c|}
\hline \multirow{2}{*}{ Enlace } & \multicolumn{4}{|c|}{$\tau_{\text {rms }}(\boldsymbol{n s})$} \\
\cline { 2 - 5 } & \multicolumn{2}{|c|}{ De Pie } & \multicolumn{2}{c|}{ Tumbado } \\
\cline { 2 - 5 } & Sujeto 1 & Sujeto 2 & Sujeto 1 & Sujeto 2 \\
\hline TO_L & 4.13 & 4.40 & 5.41 & 6.40 \\
\hline AB_R & 3.65 & 3.35 & 8.48 & 8.97 \\
\hline FA_R & 4.25 & 3.78 & 7.08 & 4.23 \\
\hline KN_L & 2.63 & 4.18 & 6.51 & 8.21 \\
\hline AN_L & 3.48 & 4.54 & 8.72 & 8.62 \\
\hline Promedio & 3.63 & 4.05 & 7.24 & 7.29 \\
\hline
\end{tabular}

Tabla 3.11. rms delay spread a $30 \mathrm{~dB}$ para las posiciones de $\mathrm{Rx}$ sobre el cuerpo de los sujetos en postura de pie y tumbado.

\begin{tabular}{|c|c|c|c|c|}
\hline \multirow{2}{*}{ Enlace } & \multicolumn{3}{|c|}{$\bar{c}$} \\
\cline { 2 - 5 } & \multicolumn{2}{|c|}{ De Pie } & \multicolumn{2}{c|}{ Tumbado } \\
\cline { 2 - 5 } & Sujeto 1 & Sujeto 2 & Sujeto 1 & Sujeto 2 \\
\hline TO_L & 30 & 32 & 37 & 37 \\
\hline AB_R & 31 & 24 & 48 & 59 \\
\hline FA_R & 30 & 29 & 45 & 28 \\
\hline KN_L & 22 & 29 & 32 & 40 \\
\hline AN_L & 27 & 28 & 41 & 50 \\
\hline Promedio & 28 & 28 & 41 & 43 \\
\hline
\end{tabular}

Tabla 3.12. Número de componentes multicamino recibidas para cada posición de Rx sobre el cuerpo en postura de pie y tumbado.

En promedio, la variación tanto del $\mathrm{rms}$ delay spread como del número de componentes multicamino recibido medido en el canal off-body para el caso de un sujeto en posición tumbada es aproximadamente del doble que el valor observado para el caso de un sujeto en posición de pie. Las posiciones que menor variación sufren respecto al 
ensanchamiento de retardo en el canal entre posturas son TO_L y FA_R con incrementos del $31 \%$ y $66 \%$ para el caso del sujeto 1 y del $45 \%$ y el $12 \%$ para el sujeto 2 . El resto de posturas se ha observado que presentan una alta variabilidad y dependencia con el número medio de componentes multicamino recibidas el cual es en promedio entre un $46 \%$ (sujeto 1 ) y un $53 \%$ (sujeto 2) superior para el caso de los sujetos en posición tumbada respecto a la posición de pie.

\subsubsection{Modelo de canal CM4 UWB}

El modelo de respuesta al impulso propuesto para el canal off-body (CM4) UWB en el estándar IEEE 802.15.6 considera, al contrario que en otros estándares como el IEEE 802.15.4 [IEEE 802.15.4, 2004], una variación del modelo Saleh-Valenzuela (S-V) en el cual las diferentes componentes multicamino recibidas se pueden agrupar en un único cluster. Por este motivo, el modelo se simplifica respecto al S-V original eliminando la caracterización del tiempo de llegada de componentes intra-cluster, dejando únicamente el tiempo inter-cluster. De esta forma, se plantea un modelo de respuesta a impulso de siguiente forma,

$$
h(\tau)=\sum_{l=0}^{L-1} \alpha_{l} \delta\left(\tau-\tau_{l}\right)
$$

donde $\alpha_{l}$ y $\tau_{l}$ son, respectivamente, la amplitud de la componente multicamino l-ésima y el retardo respecto al instante temporal de transmisión $t . \delta(\cdot)$ es la función delta.

En unidades lineales, la distribución de amplitudes sigue una ley exponencial decreciente caracterizada por el factor de decaimiento $\Gamma$ y el valor de la distribución Rice $K$ que modela la diferencia entre la amplitud de la componente dominante (componente en $\tau=0$ ) y la amplitud promedio del resto de componentes. De esta forma el modelado de la amplitud se realiza de la siguiente forma,

$$
\left|\alpha_{l}\right|^{2}=e^{-\frac{\tau_{l}}{\Gamma}-k[1-\delta(l)]} \beta
$$

donde $\Gamma$ es el factor de decaimiento exponencial, $k$ caracteriza la influencia del parámetro $K$ de la distribución Rice, el cual puede calcularse como $k=\Delta k(\ln (10) / 10)$ donde $\Delta k$ es la diferencia en $\mathrm{dB}$ entre el nivel de potencia de la contribución más significativa respecto de la media del resto de contribuciones. $\delta(\cdot)$ es la función delta, la cual vale 0 para cualquier valor de $l$ distinto de 0 . Por último $\beta$ es un término que modela la dispersión de los valores 
respecto de la media y estadísticamente se modela por medio de una distribución normal de media 0 y desviación típica $\sigma_{\beta}\left(\beta: N\left(0, \sigma_{\beta}\right)\right)$. La obtención de los valores para los parámetros de la distribución de amplitudes según el modelo anterior, se puede reducir a un ajuste lineal considerando el logaritmo neperiano de las amplitudes de las componentes multicamino recibidas, de la siguiente forma,

$$
\ln \left(\left|\alpha_{l}\right|^{2}\right)=\left\{\begin{array}{l}
0 \quad l=0 \\
\ln \left(e^{-\frac{\tau_{l}}{\Gamma}-k}\right) \quad l>0
\end{array}\right.
$$

A partir de lo anterior, el ajuste se puede realizar considerando la siguiente transformación,

$$
\ln \left(\left|\alpha_{l}\right|^{2}\right)=-\frac{\tau_{l}}{\Gamma}-k \rightarrow y=-a \cdot x-b
$$

El ajuste lineal se ha realizado a partir de las respuestas al impulso del canal $h(\tau)$ medidas para cada uno de los enlaces entre la antena transmisora y la receptora sobre los cinco puntos de colocación en el cuerpo de los sujetos. Con el propósito de realizar un promediado de todas las respuestas al impulso obtenidas para cada uno de los puntos de colocación de Rx sobre el cuerpo de los sujetos, diferenciando por cada una de las posturas, se ha realizado un proceso previo de normalización tanto en amplitud como en retardo sobre cada una de estas. La amplitud de cada una de las respuestas se ha normalizado a 1 y se ha desplazado el retardo de la contribución más significativa a $\tau_{0}=0$. Una vez realizada esta normalización, se ha calculado el PDP como $\left|h_{\text {norm }}(\tau)\right|^{2}$. Para cada grupo de PDPs normalizados, correspondientes a cada postura considerada, se ha calculado el perfil de potencia-retardo promedio (APDP, Averaged Power Delay Profile) de la siguiente forma,

$$
\begin{gathered}
A P D P_{\text {pie }}(\tau)=\frac{1}{10}\left(\sum_{p=1}^{5} P D P_{\text {S1 pie }}^{p}(\tau)+\sum_{p=1}^{5} P D P_{S 2 \text { pie }}^{p}(\tau)\right) \\
A P D P_{\text {tumb }}(\tau)=\frac{1}{10}\left(\sum_{p=1}^{5} P D P_{\text {S1tumb }}^{p}(\tau)+\sum_{p=1}^{5} P D P_{\text {S2tumb }}^{p}(\tau)\right)
\end{gathered}
$$

donde el índice $p$ indica cada una de las 5 posiciones de colocación de Rx sobre el cuerpo del sujeto. En la Figura 3.16 se muestra el APDP para cada una de las posturas consideradas. 


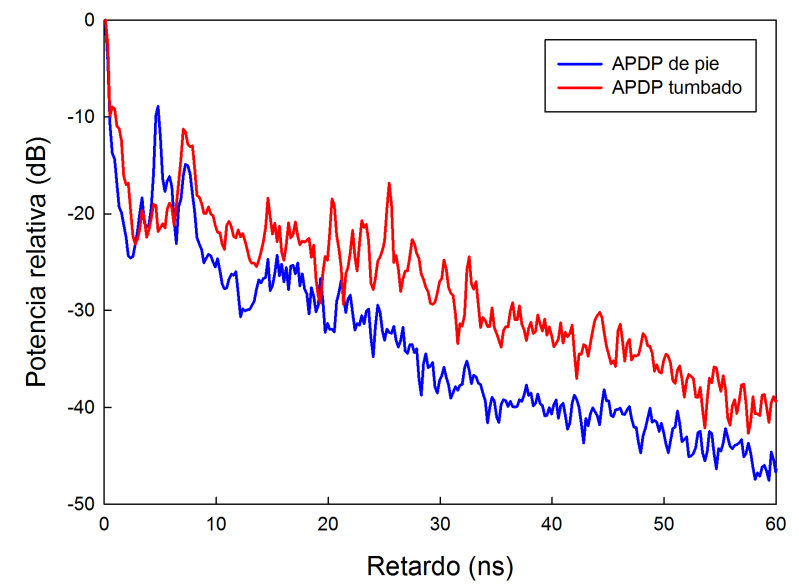

Figura 3.16: APDP normalizados para cada postura de los sujetos.

A partir de cada uno de los perfiles de potencia-retardo promediados obtenidos anteriormente se ha obtenido el factor de decaimiento $\Gamma$ y el valor del parámetro Rice $k$ para el modelo de distribución de amplitudes teniendo en cuenta el cambio indicado en la expresión (3.10). En la Figura 3.17 se muestra el ajuste realizado sobre cada uno de los APDP para cada postura considerada.

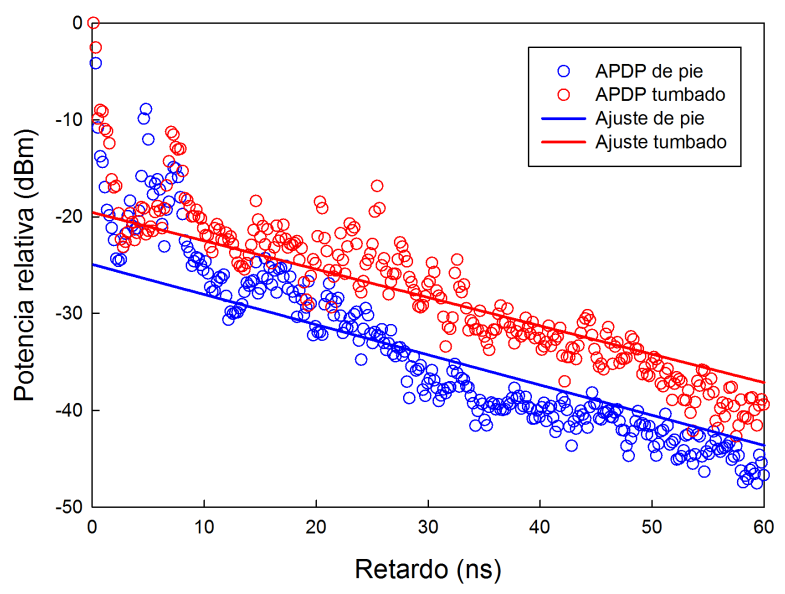

Figura 3.17. Ajuste lineal de los perfiles de potencia-retardo.

En el caso del ajuste de la distribución de amplitudes para las respuestas al impulso considerando los sujetos en posición de pie, la recta de regresión obtenida ha sido la siguiente:

$$
\ln \left(|\alpha(\tau)|^{2}\right)=-0.0718 \cdot \tau-5.74
$$

El error cometido en el ajuste, cuantificado a partir de la raíz cuadrada del error cuadrático medio (RMSE, Root Mean Square Error), es 0.792. Por otro lado, la recta de ajuste de la distribución de amplitudes para el caso de los sujetos tumbados ha sido la siguiente: 


$$
\ln \left(|\alpha(\tau)|^{2}\right)=-0.0675 \cdot \tau-4.50
$$

El valor del RMSE en este caso es 0.636 .

En la Tabla 3.13 se resumen los parámetros del modelo de amplitudes para la respuesta al impulso obtenidos a partir del proceso de ajuste lineal anterior.

\begin{tabular}{|c|c|c|}
\hline Postura & $\Gamma(\mathbf{n s})$ & $\boldsymbol{k}(\Delta \boldsymbol{k})$ \\
\hline De pie & 13.93 & $5.74(24.93 \mathrm{~dB})$ \\
\hline Tumbado & 14.82 & $4.50(19.54 \mathrm{~dB})$ \\
\hline
\end{tabular}

Tabla 3.13. Parámetros del modelo de ajuste lineal de los APDP según postura.

Se observa que en ambos casos, el factor de decaimiento es similar, mientras que la diferencia entre la componente más significativa y el resto de componentes, modelado a partir del parámetro $k$ de la distribución Rice, es inferior en el caso de los sujetos tumbados. Esto se puede justificar por el hecho de que, debido a la posición relativa en elevación entre Tx y Rx para cada enlace sobre el cuerpo, por un lado, las pérdidas por alineamiento entre las antenas son similares en todos los enlaces; y por otro, el nivel de shadowing debido a la obstrucción por partes del cuerpo es menor al caso de los sujetos de pie. Esto último puede causar, en primer lugar, que el número recibido de componentes multicamino en cada punto de colocación de Rx sea mayor; y en segundo lugar, que a un menor valor de retardo haya más componentes con un nivel de atenuación menor respecto a la potencia de la contribución principal.

Con el fin de modelar el shadowing para cada enlace Tx-Rx en las dos posturas consideradas, se ha realizado la caracterización estadística del parámetro $\beta$ empleando individualmente los PDPs, previamente normalizados en retardo y en amplitud, correspondientes a cada enlace para los dos sujetos en las dos posturas (10 PDPs por cada postura). De esta forma, para cada retardo $\tau_{l}$ en cada grupo de PDPs por postura, se ha calculado la dispersión respecto al ajuste lineal correspondiente obtenido a partir de las expresiones (3.13) y (3.14). Además, se han considerado únicamente como válidas aquellas componentes situadas por encima de un umbral de $-30 \mathrm{~dB}$, considerando como ruido todas las componentes por bajo de este. En la Figura 3.18 se muestra el modelado estadístico del parámetro $\beta$ para cada una de las posturas de los sujetos. 


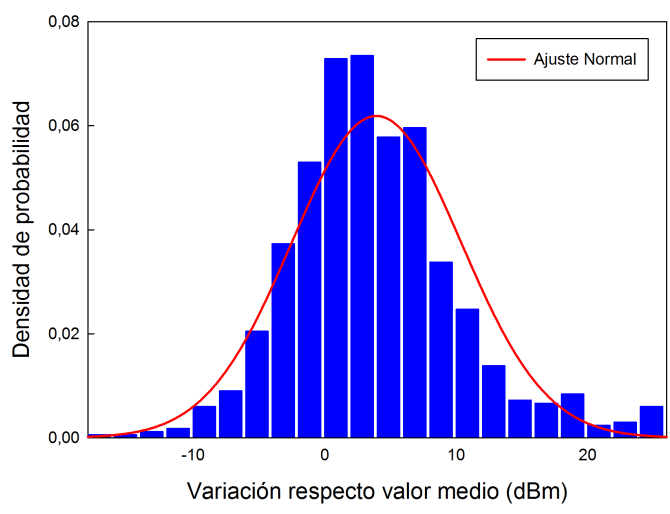

(a)

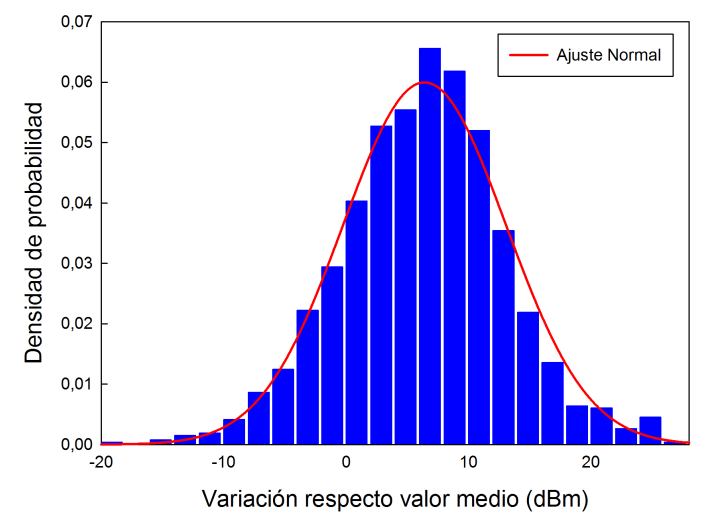

(c)

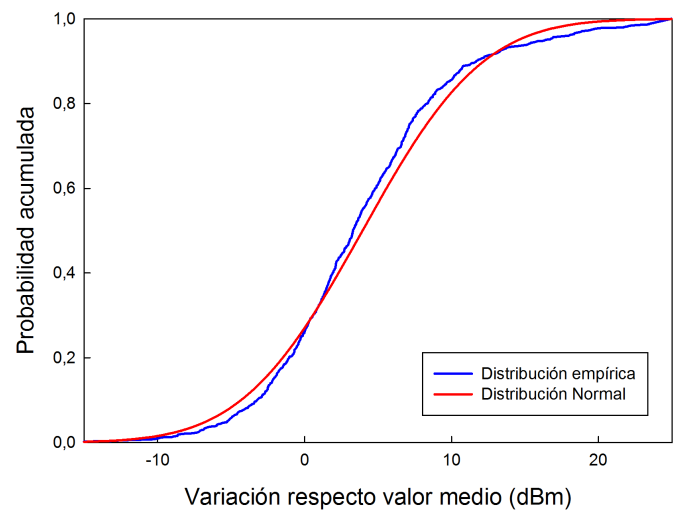

(b)

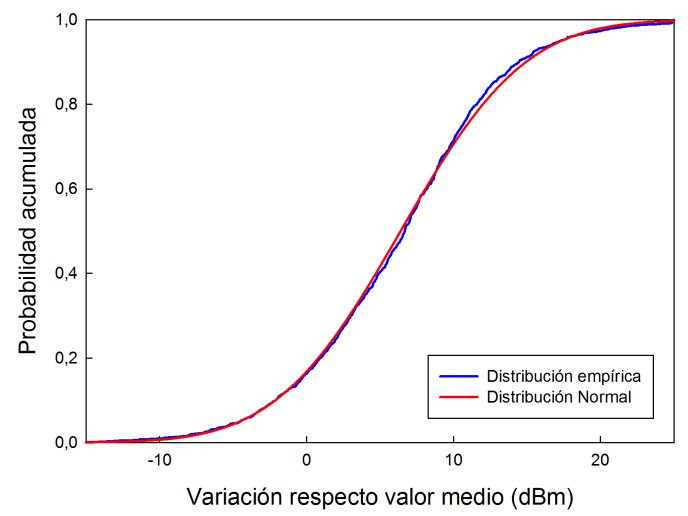

(d)

Figura 3.18: Ajuste estadístico del parámetro $\beta$. (a) función de distribución para sujetos de pie, (b) función de probabilidad acumulada para sujetos de pie, (c) función de distribución para sujetos tumbados, (d) función de probabilidad acumulada para sujetos tumbados.

Tal y como se observa, el modelado estadístico del parámetro de shadowing $\beta$ se puede realizar a partir del ajuste por medio de una función de distribución gaussiana o Normal (log-normal en el dominio logarítmico). Para el caso del shadowing en el canal off-body correspondiente a los enlaces para los sujetos en posición de pie, el ajuste se puede realizar por medio de una distribución Normal de parámetros $\mu_{\beta}=3.94$ y $\sigma_{\beta}=6.45$ con un error de ajuste, cuantificado a partir del RMSE de 0.03. De igual forma, para el caso de los sujetos en posición tumbada, el ajuste se puede realizar a partir de una función de distribución Normal de parámetros $\mu_{\beta}=6.39$ y $\sigma_{\beta}=6.65$, con un valor de RMSE de 0.01 .

La caracterización estadística de los parámetros del modelo de distribución de llegadas de componentes multicamino se ha realizado, al igual que en caso anterior, considerando individualmente cada respuesta $h(\tau)$ medida para cada enlace Tx-Rx en cada sujeto para cada una de las posturas consideradas con el fin de tener suficiente variedad estadística. En el modelo CM4 para UWB propuesto en estándar IEEE 802.15.6 el tiempo de 
llegada de las componentes se puede modelar por medio de una distribución de Poisson con tasa $\lambda$ y número medio de componentes recibidas $\bar{L}$, de la siguiente forma,

$$
\begin{gathered}
p\left(\tau_{l} \mid \tau_{l-1}\right)=\lambda \exp \left(-\lambda\left(\tau_{l}-\tau_{l-1}\right)\right) \\
p(L)=\frac{\bar{L}^{L} \exp (\bar{L})}{L !}
\end{gathered}
$$

El valor de cada uno de los parámetros se ha calculado por medio de un método de búsqueda de picos desarrollado e implementado en Matlab en el cual, considerando a la entrada un PDP y un valor de potencia umbral para tener en cuenta solo las componentes cuyo nivel de potencia relativo está a un cierto valor por bajo de $0 \mathrm{~dB}$, fijado a $-30 \mathrm{~dB}$ en este caso, se obtiene los valores de potencia de los picos correspondientes a cada componente multicamino con nivel de potencia significativo, su posición en retardo y un vector con las diferencias entre tiempos de llegada $\tau_{l}-\tau_{l-1}$. En la Figura 3.19 se muestra el modelado estadístico de la distribución de tiempo de llegada de componentes a partir de los datos obtenidos por medio del método anterior. Así mismo, el tiempo entre llegadas se observa que puede modelarse por medio de una distribución de Poisson con parámetro $\lambda=0.95$ con un valor de RMSE de 0.135 para el caso de los sujetos en posición de pie y $\lambda=1.08$ con un RMSE de 0.135 para sujetos tumbados.

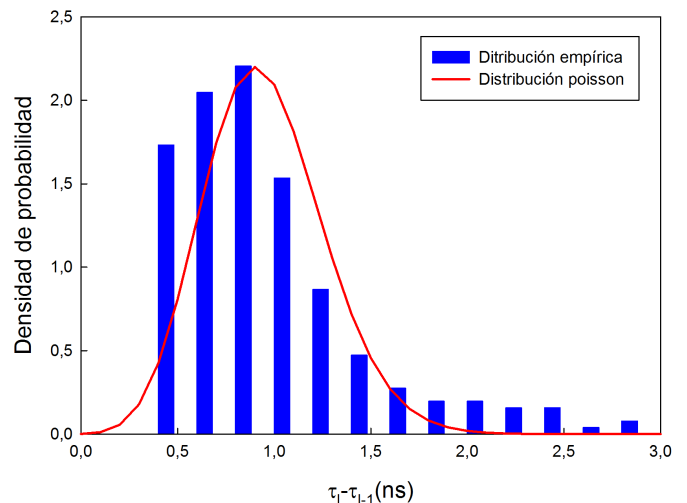

(a)

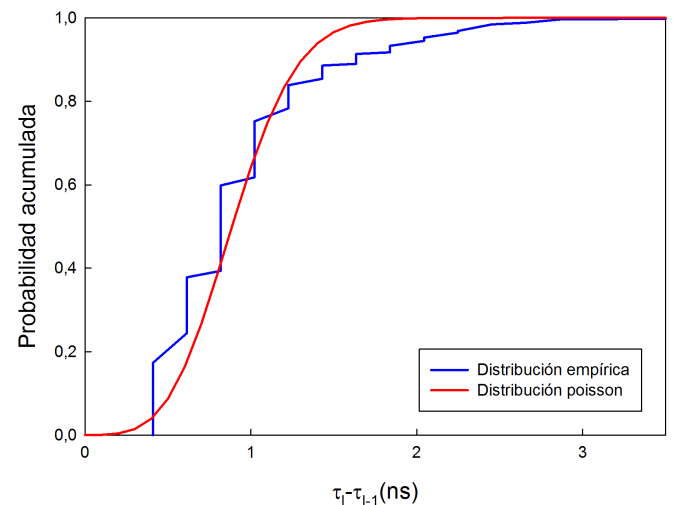

(b) 


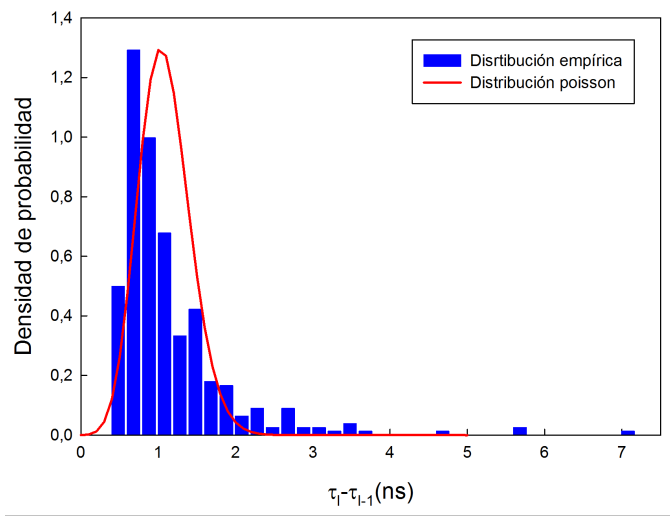

(c)

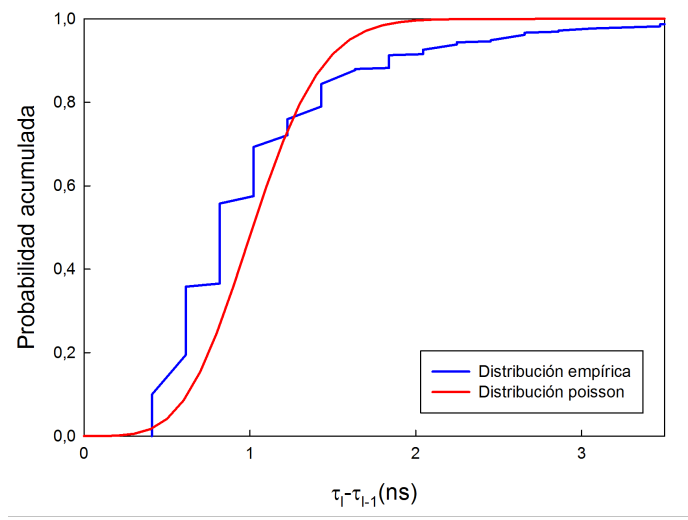

(d)

Figura 3.19: Ajuste estadístico del tiempo entre llegadas según modelo de Poisson. (a) función de distribución para sujetos de pie, (b) función de probabilidad acumulada para sujetos de pie, (c) función de distribución para sujetos tumbados, (d) función de probabilidad acumulada para sujetos tumbados.

Respecto al valor medio de componentes multicamino recibidas, se ha considerado al igual que en el caso anterior, un umbral de $-30 \mathrm{~dB}$ bajo la componente de mayor potencia (normalizada a $0 \mathrm{~dB}$ ). El número de componentes multicamino recibido $\bar{L}$ correspondiente a cada una de las posturas consideradas es $\bar{L}=27$ para sujetos de pie y $\bar{L}=39$ para sujetos tumbados.

En la Tabla 3.14 se resumen los valores obtenidos para los parámetros del modelo CM4 propuesto en el estándar IEEE 802.15.6 para la banda de UWB, obtenidos a partir del modelado de la respuesta al impulso del canal off-body entre una antena Tx y el conjunto de cinco puntos de colocación de una antena sobre el cuerpo de los sujetos, considerando estos en posición de pie y tumbados.

\begin{tabular}{|c|c|c|}
\hline \multirow{2}{*}{ Parámetro } & \multicolumn{2}{|c|}{ Postura } \\
\cline { 2 - 3 } & De pie & Tumbado \\
\hline$\Gamma(\boldsymbol{n} \boldsymbol{s})$ & 13.93 & 14.82 \\
\hline $\boldsymbol{k}(\Delta \boldsymbol{k}(\boldsymbol{d B}))$ & $5.74(24.93 \mathrm{~dB})$ & $4.50(19.54 \mathrm{~dB})$ \\
\hline $\boldsymbol{\sigma}_{\boldsymbol{\beta}}(\boldsymbol{d B})$ & 6.45 & 6.65 \\
\hline$\lambda(\boldsymbol{n} \boldsymbol{s})$ & 0.95 & 1.08 \\
\hline$\overline{\boldsymbol{L}}$ & 27 & 39 \\
\hline
\end{tabular}

Tabla 3.14. Parámetros obtenidos para el modelo CM4 UWB considerando sujetos de pie y tumbados.

Debido a la ausencia de estudios en los que se contemple la obtención de los parámetros del modelo CM4 en UWB dependiendo de la postura adoptada por los sujetos, únicamente se puede establecer una comparación con los valores para el modelo de canal correspondiente a sujetos de pie. Respecto al valor obtenido para la tasa de decaimiento $\Gamma$ (13.93 ns), mientras que el valor reportado en [Di Bari et al., 2013] es similar (11.3 ns), en 
ambos casos difiere significativamente del reportado en [IEEE P802.15-08-0780-09-0006, 2009] (44.6 ns). El valor obtenido correspondiente al modelado del factor $k$ ( $24.93 \mathrm{~dB})$ es similar al reportado en los dos estudios anteriores [22.2-26.2 dB]. Respecto al modelado del término de shadowing, en [Di Bari et al., 2013] el mejor ajuste se realiza por medio de la distribución log-normal con un valor de desviación típica $\sigma_{\beta}=7.1 \mathrm{~dB}$, similar al ajuste realizado en el presente estudio sobre la misma distribución pero con desviación típica $\sigma_{\beta}=$ $6.45 \mathrm{~dB}$.

El parámetro que mayor diferencia presenta es el número medio de componentes multicamino recibidas. Se ha reportado un valor de $\bar{L}=400$ en [IEEE P802.15-08-0780-090006, 2009] y $\bar{L}=132$ en [Di Bari et al., 2013] frente a los 27 obtenidos en el presente estudio. Esto puede deberse a las mayores dimensiones del entorno donde se realizaron las medidas o el umbral de potencia bajo la contribución principal considerado.

Por último, respecto al modelado del tiempo entre llegada de componentes, el modelado presentado en [Di Bari et al., 2013] se realiza por medio de una distribución gaussiana inversa (INVG), por lo que los valores no son comparables con los obtenidos en el presente estudio, y en [IEEE P802.15-08-0780-09-0006, 2009] el modelado propuesto es por medio de la distribución de Poisson pero no se presentan valores de referencia.

\subsection{Conclusiones}

En el presente capítulo se ha presentado un estudio de la caracterización del canal de propagación WBAN off-body en la banda de UWB considerando condiciones de propagación por visión directa (LOS) y canal estático, desde la perspectiva de la influencia del entorno, la posición de colocación de una antena sobre el cuerpo y la postura adoptada por los sujetos. A pesar de que el tamaño y estructura del entorno influye en que exista un mayor número de componentes recibidas con un nivel significativo de potencia a mayores retardos (componentes con potencia por encima de los $-90 \mathrm{~dB}$ hasta los $44 \mathrm{~ns}$ a $3 \mathrm{~m}$ para el TSC5 frente a $35 \mathrm{~ns}$ en el SiCoMo), en condiciones de visión directa el tamaño del entorno en el canal de propagación entre un dispositivo colocado en la superficie del cuerpo y otro exterior operando como punto de acceso es poco significativo. Respecto al canal de propagación sin influencia del cuerpo, en el caso de las antenas sobre el mismo, y para ambos entornos de medida, se ha observado tanto un aumento de $2 \mathrm{~dB}$ en el valor de las pérdidas de propagación como una disminución del $41 \%$ en la dispersión promedio del valor del rms delay spread $\tau_{r m s}$. 
Respecto a la variación en las características de propagación de los enlaces off-body según la posición de colocación de la antena sobre el cuerpo, se ha observado una alta variabilidad con el punto de colocación considerado. Las posiciones de colocación ubicadas en la parte alta del cuerpo se ha observado que presentan mayores pérdidas que las bajas, debido al menor número de componentes recibidas por reflexiones en el entorno pero, por el contrario, son las más estables respecto a la dispersión temporal del canal. Se ha determinado que el punto de colocación correspondiente al área del pecho es el más estable de los considerados. Por último, se ha observado un incremento en promedio entre el $46 \%$ y el 53\% en el número de componentes multicamino recibidas por posición de Rx sobre el cuerpo para el caso de sujetos en posición tumbada respecto a de pie. Así mismo, la variación media del ensanchamiento de retardo por posición de colocación de Rx según la postura se ha encontrado ser cercana al doble y presentar una alta variabilidad en ambas posturas consideradas. Además, se han obtenido valores para los parámetros estadísticos del modelo de respuesta al impulso CM4 para UWB propuesto en el IEEE 802.15.6 similares en ambas posturas consideradas. La principal variación observada ha sido la disminución en la diferencia de potencia entre la contribución principal y la potencia media del resto, en el caso de los sujetos tumbados (19.54 dB frente a $24.93 \mathrm{~dB}$ ). En ambos casos se ha obtenido un buen ajuste estadístico sobre una distribución normal del modelo de shadowing y Poisson para la distribución de tiempos de llegada de componentes. En general se puede concluir que la variación en el canal de propagación off-body UWB debida a la postura de los sujetos es inferior al efecto sobre los parámetros de propagación debido al punto de colocación de la antena sobre el cuerpo. 


\section{Capítulo 4 \\ Caracterización Dinámica del Canal In-Body}

En este capítulo se presentan los resultados de la caracterización del canal de propagación entre dos dispositivos, uno de ellos ubicado en el interior del cuerpo y otro colocado en tres posiciones respecto al anterior: sobre la superficie del mismo (canal in-body a on-body o IB2OB), en el exterior a cierta distancia de separación (canal in-body a off-body o IB2OFF) y en el interior del cuerpo (canal in-body a in-body o IB2IB), considerando el movimiento relativo entre ambos inducido por la respiración de un sujeto. Los efectos sobre el canal radio debidos a la variación en el tiempo de la distancia relativa entre dispositivos se ha analizado por un lado en términos de la dispersión en frecuencia o efecto Doppler, cuantificada a partir de los valores de ensanchamiento y modelado de la forma del espectro Doppler, y por otro en términos de la estabilidad temporal del canal radio caracterizada por medio de la función de autocorrelación y el tiempo de coherencia. 


\subsection{Estado del arte}

La caracterización de la propagación de señales desde el interior del cuerpo presenta serias limitaciones debidas tanto a la dificultad del modelado de la propagación a través de los tejidos y la variación en las características de radiación de las antenas por la interacción en campo cercano con el cuerpo, como por el hecho de que, en la mayoría de los casos no es posible realizar medidas sobre sujetos o animales vivos debido a restricciones éticas. Actualmente, el número de estudios in vivo es muy limitado y los existentes, como los presentados en [Santiago y Balasingham, 2013] y en [Floor at al., 2015], llevan a cabo la caracterización del canal in-body mediante medidas sobre especímenes de cerdos, debido a la similitud en las propiedades dieléctricas de los tejidos de estos con los humanos. La mayoría de estudios de la propagación en el canal in-body se plantean desde la perspectiva del modelado electromagnético empleando modelos digitales (phantoms numéricos), usualmente de partes concretas del cuerpo, en los cuales se considera o bien un único tejido (modelos homogéneos) o varios (heterogéneos), cada uno con una cierta permitividad relativa y conductividad, cuyos valores son dependientes de la frecuencia de operación [Khaleghi et al., 2012]. Esta dependencia, habitualmente se incluye en las simulaciones por medio de simplificaciones realizadas sobre las ecuaciones Cole-Cole, como las utilizadas en [Khaleghi et al., 2010]. Otra posibilidad a la hora de estudiar la propagación in-body es el empleo de materiales (phantoms físicos) diseñados para emular el comportamiento dieléctrico de determinados tejidos humanos dentro de un rango de frecuencia, como el phantom líquido empleado en [Santiago et al., 2014] o el formado por diferentes capas de tejido abdominal porcino en [Thotahewa et al., 2015].

En el estándar IEEE 802.15.6 la banda de frecuencias propuesta para su utilización en dispositivos implantados es principalmente la banda Medical Implant Communication Service (MICS) de $402 \mathrm{MHz}$ a $405 \mathrm{MHz}$ [IEEE 802.15.6, 2012], [Santiago et al., 2013c]. Aunque esta banda presenta unas pérdidas de propagación bajas, el reducido ancho de banda disponible $(300 \mathrm{kHz})$ hace que el ámbito de aplicación se limite casi por completo a la telemetría y por lo tanto sea insuficiente para aplicaciones en las que se requiera una tasa de transferencia de datos superior. Para salvar esta limitación y debido a las características inherentes de la tecnología UWB, tales como baja potencia de emisión, alto ancho de banda y reducidas dimensiones de los dispositivos, desde hace unos años está captando un gran interés en su aplicación a enlaces con implantes para aplicaciones donde se requiera una alta tasa de transferencia. En este sentido, la aplicación de referencia en la cual se basan la mayoría de estudios sobre propagación in-body en UWB existentes es la transmisión de video en tiempo real desde una cápsula endoscópica inalámbrica (WCE, Wireless Capsule Endoscopy) 
[Toennies et al., 2010]. Este tipo de cápsulas incluyen en un dispositivo de muy reducidas dimensiones, diseñado para poder ser ingerido por una persona, tanto los elementos de captación de video, iluminación, transmisión y alimentación. El modo de operación habitual de este tipo de dispositivos es la transmisión a una unidad receptora colocada en la zona abdominal de un sujeto de las fotografías obtenidas durante su paso por las diferentes zonas del tracto gastrointestinal. Estas imágenes son postprocesadas por software y convertidas en video. En la actualidad los dispositivos comerciales, como las PillCams ${ }^{\mathrm{TM}}$, operan en la frecuencia de 403-434 MHz con tasas de transferencia de $2.7 \mathrm{Mbps}$, las cuales son insuficientes para ofrecer la tasa de transferencia de datos necesaria para la transmisión de video en tiempo real de alta resolución.

La caracterización del canal de propagación in-body en los diferentes estudios existentes sobre UWB mayoritariamente se centran en el modelado de las pérdidas de propagación y muy pocos presentan modelos del comportamiento dispersivo temporal del canal. Además, debido a la heterogeneidad de la morfología humana, es necesario extender el estudio a múltiples partes del cuerpo ya que los resultados obtenidos para una parte concreta del cuerpo pueden no ser extrapolables a otras.

En la actualidad, la mayoría de estudios sobre propagación hacen referencia a enlaces entre implantes en la zona abdominal (para WCE) o la pectoral (para implantes cardiacos). Pocos estudios como el presentado en [Duncan y Cummings, 2013], en el cual se analiza mediante simulaciones el enlace entre un implante a $6 \mathrm{~mm}$ en la parta alta del brazo y un dispositivo externo en términos de potencia transmitida, tasa de absorción específica o SAR y pérdidas de propagación, se centran en otras áreas del cuerpo. En [Khaleghi et al., 2011] se presenta un estudio del canal in-body por medio de simulaciones en la banda de 1-6 GHz para implantes en el pecho a dos profundidades $(20 \mathrm{~cm}$ y $80 \mathrm{~cm}$ ). Los autores presentan tanto un modelo de pérdidas de propagación lineal, con exponente de pérdidas 0.85 y un modelo de respuesta al impulso en el cual tanto la distribución de amplitudes de las componentes multicamino como el tiempo entre llegadas se observa que sigue una estadística normal o gaussiana. Además, los autores han observado que la dispersión de retardo en el canal in-body es muy pequeña $(\approx 1 \mathrm{~ns}$ ), en comparación con otros entornos como indoor. En [Santiago et al., 2014] se realiza un estudio de viabilidad del empleo de UWB en in-body considerando diferentes separaciones entre dos antenas implantadas. Las medidas se realizan empleando un phantom líquido y se concluye que la banda de 3.1-4.6 GHz es la más recomendable para establecer enlaces UWB (suponiendo un ancho de banda de $500 \mathrm{MHz}$ ) manteniendo un nivel de pérdidas asumible para distancias de hasta $100 \mathrm{~mm}$. En [Santiago y Balasingham, 2013] se presenta un estudio tanto teórico como experimental, empleando un ejemplar de cerdo, de la 
propagación en UWB para la transmisión de video en tiempo real desde una cápsula endoscópica. Para lo cual, por medio de un procedimiento quirúrgico, se implantó un dispositivo transmisor en el tracto gastrointestinal del animal. Se observa que no es posible establecer enlaces por encima de 80 Mbps a profundidades superiores a $33 \mathrm{~mm}$ pero empleando compresión y a una tasa de 1 Mbps es posible realizar enlaces de hasta $120 \mathrm{~mm}$. En este estudio se concluye que para alcanzar los 150-200 mm necesarios en un escenario real, se requiere emplear diferentes soluciones técnicas adicionales. En [Santiago y Balasingham, 2013b], a partir del modelo pérdidas desarrollado en [Støa et al., 2010] y considerando una sensibilidad de $80 \mathrm{~dB}$ en el receptor se determina que conforme aumenta la distancia entre el implante y la superficie, el nivel de potencia de transmisión debe aumentarse para poder alcanzar la banda de frecuencias estandarizada en el IEEE 802.15.6 para UWB. En el caso de una distancia de $200 \mathrm{~mm}$, es necesario un nivel de potencia de 10 dBm para operar como máximo a la frecuencia de $3163.4 \mathrm{MHz}$.

Aunque la caracterización de la propagación en entornos in-body desarrollada en los diferentes estudios existentes asumen condiciones estáticas del canal, el movimiento relativo entre transmisor y receptor puede dar lugar a variaciones temporales y desplazamiento en frecuencia de la señal recibida (efecto Doppler). Los efectos sobre el canal radio debidos al movimiento del cuerpo han sido estudiados por diferentes autores principalmente para el canal on-body, como en [Smith et al., 2009] y [Liu et al., 2013] para banda estrecha o en [D'Errico y Ouvry, 2011] y [Fu et al., 2011] para banda estrecha y UWB, y puntualmente para otros canales como el body-body en [Oestges, 2015], pero hasta el momento no hay estudios de este tipo para el canal in-body.

En ausencia de movimiento del cuerpo debido a cambios en la postura, posición de las extremidades, etc., la respiración es la principal causa de variación en la distancia relativa entre dispositivos ubicados en la zona pectoral y abdominal del cuerpo tanto externa como interna. En el campo de las WBAN los efectos sobre el canal debidos a la respiración han sido poco tratados en la literatura. El único estudio del cual tenemos constancia es [Petrillo et al., 2015] en el cual se realiza un modelado del efecto de la respiración en el canal on-body entre dispositivos a $60 \mathrm{GHz}$ en el interior de una cámara anecoica. Por lo tanto el objetivo del presente capítulo es realizar la caracterización del efecto que tiene la respiración humana en el canal radio considerando los escenarios de propagación in-body a on-body (IB2OB), in-body a off-body (IB2OFF) e in-body a in-body (IB2IB), tal y como se puede ver en la Figura 2.9, en los cuales al menos uno de los dispositivos de la red de área corporal se encuentra ubicado en el interior del cuerpo. Los efectos derivados del movimiento entre dispositivos se han estudiado desde la perspectiva de la caracterización del efecto Doppler, a partir del 
ensanchamiento del espectro de potencia Doppler y el modelado de la forma de este, y por otro lado, desde la perspectiva de la tasa de variación en el tiempo del canal radio, modelada a partir de la autocorrelación y el tiempo de coherencia.

\subsection{Esquema de medidas del canal de propagación in-body en condiciones dinámicas}

El esquema general de los elementos empleados para realizar las medidas en frecuencia del canal de propagación bajo condiciones dinámicas, debidas al movimiento relativo entre transmisor $(\mathrm{Tx})$ y receptor $(\mathrm{Rx})$ causado por la respiración, considerando al menos un dispositivo en el interior del cuerpo se detalla en la Figura 4.1.

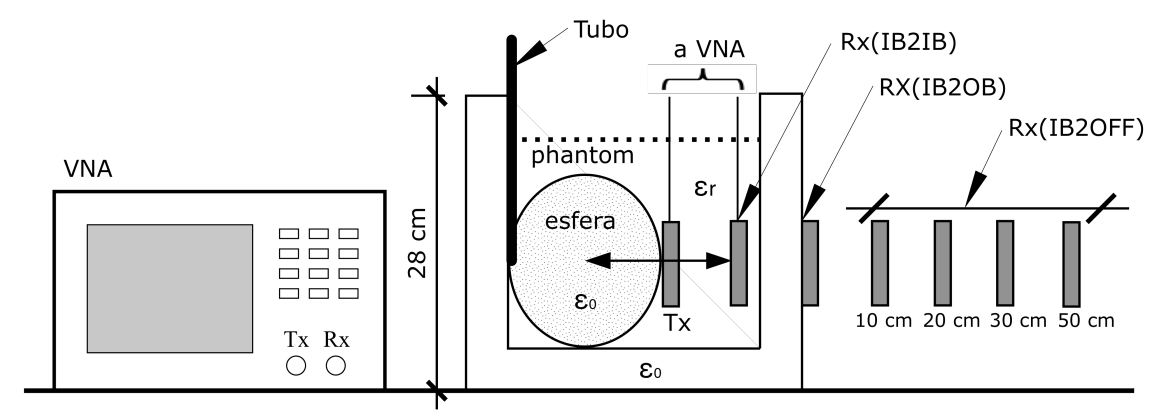

Figura 4.1: Esquema general de elementos para medidas en frecuencia del canal in-body dinámico debido al efecto de la respiración.

Los tres escenarios de propagación contemplados son los siguientes:

- In-body a on-body (IB2OB): un dispositivo ubicado en el interior del cuerpo y uno sobre la superficie del mismo.

- In-body a off-body (IB2OFF): un dispositivo ubicado en el interior del cuerpo y uno en el exterior y a una cierta distancia de separación.

- In-body a in-body (IB2IB): dos dispositivos ubicados en el interior del cuerpo.

Durante las medidas realizadas considerando cada uno de los escenarios de propagación anteriores, se ha simulado la cavidad torácica humana por medio de un recipiente construido en poliestireno expandido (EPS) de dimensiones $28 \times 28 \times 28 \mathrm{~cm}^{3}$ con una pared de $4 \mathrm{~cm}$, relleno de con un phantom líquido [Zhou et al., 2006] diseñado para emular las propiedades dieléctricas de los tejidos humanos con alto contenido en agua (como los músculos) en la banda de UWB entre las frecuencias de $3.1 \mathrm{GHz}$ y $10.6 \mathrm{GHz}$. Los detalles del phantom empleado pueden consultarse en el Apéndice B. En la Figura 4.2 se muestra una imagen del contenedor con el phantom líquido en su interior. 


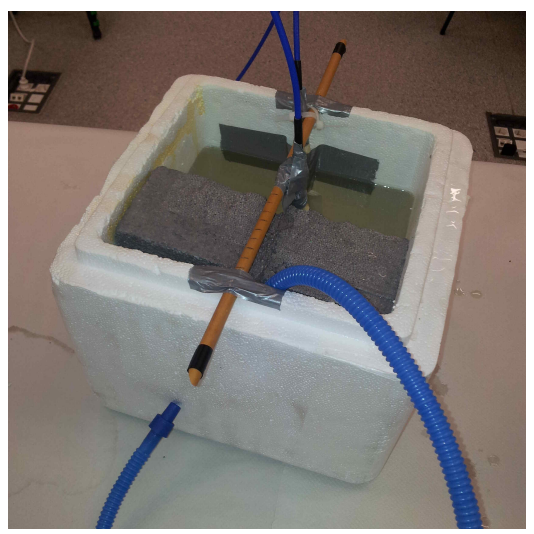

Figura 4.2: Phantom líquido y contenedor.

Las medidas del canal radio se han realizado en el dominio de la frecuencia empleando un analizador de redes vectorial (VNA) Agilent ENA E5072A controlado mediante un software desarrollado en Matlab por el equipo del Instituto de Telecomunicaciones y Aplicaciones Multimedia (iTEAM) en la Universidad Politécnica de Valencia (UPV). Para cada uno de los escenarios de propagación descritos anteriormente se ha llevado a cabo la caracterización dinámica del canal radio con el objetivo de modelar la influencia que la variación en el tiempo de la distancia de separación entre dos dispositivos (al menos uno de ellos en el interior del cuerpo) tiene en el canal de propagación, en términos del desplazamiento Doppler y el tiempo de coherencia. Los detalles de la configuración del VNA se detallan en la Tabla 4.1.

\begin{tabular}{|c|c|}
\hline Potencia Tx & $5 \mathrm{dBm}$ \\
\hline Rango de medida & $\begin{array}{c}3.1 \mathrm{GHz}, 4.8 \mathrm{GHz}, 6 \\
\mathrm{GHz} y \\
8.5 \mathrm{GHz}\end{array}$ \\
\hline $\begin{array}{c}\text { Ancho de banda } \\
\text { intermedio (IF) }\end{array}$ & $10 \mathrm{kHz}$ \\
\hline Span & $0(\mathrm{CW})$ \\
\hline Tiempo de medida & $60 \mathrm{~s}$ \\
\hline Número de puntos & 8005 \\
\hline
\end{tabular}

Tabla 4.1: Parámetros de configuración del analizador de redes para medidas in-body en condiciones dinámicas.

El VNA se ha configurado en modo de onda continua ( $\mathrm{CW}$, Continuous Wave) para emitir una señal sinusoidal a una única frecuencia central con un tiempo de muestro de 133 muestras/segundo. Esta configuración permite medir un desplazamiento Doppler máximo $f_{m} \approx 66 \mathrm{~Hz}$. El nivel de ruido del sistema se ha comprobado que se encuentra por bajo de $-105 \mathrm{dBm}$. Tanto para transmisión como para recepción se ha empleado una antena plana de dimensiones 5 x $4.4 \mathrm{~cm}$ diseñada para operar en la banda de UWB. Los detalles se pueden consultar en el Apéndice B. Las antenas colocadas en el interior del phantom han sido previamente recubiertas mediante un envoltorio de látex, eliminando al máximo el posible 
aire en el interior, con el fin de evitar que se cortocircuiten por el contacto con la solución líquida.

El conjunto pulmones-tráquea se ha emulado empleando una esfera de látex y un tubo corrugado de $96 \mathrm{~cm}$ conectado a esta, con una válvula en el otro extremo para retener el aire en el interior del sistema. Esta válvula permite por un lado la introducción de aire en el interior de la esfera para emular el hinchado de un pulmón y por otro lado la liberación controlada del mismo para reducir el contenido de aire en el interior. Acoplada a la esfera de látex por medio de un adhesivo se ha colocado la antena transmisora previamente aislada, mientras que la antena receptora se ha colocado en el interior del phantom para las medidas del canal IB2IB, sobre la superficie exterior del contenedor, orientada hacia Tx, para las medidas del canal IB2OB y sobre un soporte a cuatro distancias de separación respecto a la superficie del contenedor $(10 \mathrm{~cm}, 20 \mathrm{~cm}, 30 \mathrm{~cm}$ y $50 \mathrm{~cm})$ para las medidas del canal IB2OFF. La expansión máxima de la antena Tx se ha restringido a $4 \mathrm{~cm}$, con el fin de considerar la expansión pectoral habitual humana en condiciones de respiración normal [Olsén et al. 2011].

\subsection{Análisis de los datos}

La caracterización del canal de propagación entre un dispositivo ubicado en el interior del cuerpo (ya sea implantado, ingerido o inyectado) y otro dispositivo situado en el interior del mismo, colocado sobre la superficie o a una cierta distancia del cuerpo, se ha realizado a partir de las medidas en frecuencia del canal radio obtenidas mediante el VNA bajo las condiciones de canal dinámico y los parámetros de configuración detallados en la Tabla 4.1.

Al igual que el comportamiento dispersivo temporal del canal causado por el efecto multicamino puede caracterizarse a partir de los efectos en el tiempo y la frecuencia causados por la recepción de réplicas de una misma señal transmitida a diferentes retardos, el carácter dispersivo en frecuencia derivado del movimiento, ya sea del transmisor o receptor, o bien de los elementos presentes en el entorno, puede modelarse de igual manera en tiempo y frecuencia a partir de los efectos derivados del desplazamiento Doppler que experimenta la señal recibida. 


\subsubsection{Caracterización del movimiento relativo entre dispositivos}

Las medidas del canal de propagación, considerando este bajo condiciones dinámicas debido al movimiento relativo entre dispositivos causado por efecto de la respiración, obtenidas por medio del VNA configurado en modo de onda continua empleando los parámetros de configuración indicados en la Tabla 4.1, corresponden con muestras tomadas a valores $\Delta t$ de la respuesta en frecuencia variable en el tiempo del canal $H(f, t)$, o más concretamente del parámetro de transmisión $S_{21}$. Además, al realizarse las medidas en banda estrecha a una única frecuencia, los valores de $S_{21}$ corresponden con las muestras a $\Delta t$ de la amplitud de la señal recibida.

Asumiendo que el canal de propagación puede considerarse de tipo estacionario en sentido amplio con scattering incorrelado (WSSUS), ya que el único factor que altera las condiciones de propagación en el canal radio es el movimiento cíclico entre Tx y Rx por efecto de la respiración y este es en sí un proceso suficientemente lento $\left(f_{\text {samp }}=133 \mathrm{~Hz} \gg\right.$ $0.2 \mathrm{~Hz}$ para el caso de 12 respiraciones/minuto), y considerando una variación en las condiciones de propagación de tipo estocástico, el estudio del movimiento relativo entre dos dispositivos puede plantearse a partir de las funciones de autocorrelación de Bello [Bello, 1996] aplicando las simplificaciones derivadas del tipo de canal (ver Apéndice A, sección A.2). Debido a que las muestras se han tomado a una frecuencia central fija $(\Delta f=0)$, la función de autocorrelación del canal $R_{T}(f, m ; t, s)$ se puede reducir a $R_{T}(\Delta f=0 ; t)$. A partir del estudio de la función de autocorrelación, se puede cuantificar el efecto en el tiempo causado por el desplazamiento en frecuencia Doppler debido al movimiento entre dispositivos. Este estudio se puede realizar a partir del tiempo de coherencia $T_{c}$, definido como el valor temporal de la función de autocorrelación para el cual el valor de esta baja de un cierto valor (habitualmente entre 0.5 y 0.9 ).

Calculando la transformada de Fourier de $R_{T}(\Delta f=0 ; t)$ se puede obtener el espectro de potencia Doppler $D\left(f_{c}, \rho\right)$ (ver Apéndice A, sección A.2). De manera práctica, este se puede obtener a partir del módulo al cuadrado de la respuesta en frecuencia del canal medida en banda estrecha, tal y como se detalla en el Apéndice A, sección A.2.2. A partir de este se puede cuantificar el ensanchamiento en frecuencia debido al movimiento entre dispositivos por medio del primer momento central y la raíz cuadrada del segundo momento central, denominados ensanchamiento Doppler $B_{D}$ y ensanchamiento Doppler rms respectivamente. 


\subsubsection{Filtrado de las medidas}

Con el fin de reducir el ruido presente en las muestras obtenidas de la respuesta en frecuencia del canal $H\left(f_{c}, t\right)$ para cada escenario de propagación considerado (IB2OB, IB2OFF y IB2IB) se ha aplicado un proceso de filtrado paso bajo empleando un filtro de media móvil (MA, Moving Average) con un tamaño de ventana $L=20$ muestras. Con este tamaño se consigue una reducción aproximada del nivel de ruido equivalente a $\sqrt{L}=4.5 \mathrm{~dB}$.

La elección de este tipo de filtrado se debe a la simplicidad en su implementación y su eficiencia para reducir el ruido aleatorio presente en las medidas con un compromiso entre nivel de reducción y alteración de la forma de la señal filtrada. El filtro se ha implementado en Matlab a partir de la siguiente expresión,

$$
H_{\text {filt }}\left[f_{c}, t_{i}\right]=\frac{1}{L} \sum_{j=0}^{L-1} H\left[f_{c}, t_{i-j}\right] \quad \forall i \geq L
$$

En la expresión anterior, las $L-1$ primeras muestras de $H_{\text {filt }}\left[f_{c}, t_{i}\right]$ son copia de las muestras de la respuesta original sin filtrar. Por último, la respuesta filtrada debe desplazarse hacia la izquierda $L / 2$ muestras para compensar el desplazamiento temporal debido a la ventana deslizante.

La respuesta en frecuencia del filtro se puede obtener a partir de la siguiente expresión [Smith, 1999],

$$
|H(\hat{\omega})|=\left|\frac{\sin (\hat{\omega} L / 2)}{L \sin (\hat{\omega} / 2)}\right|
$$

La representación en frecuencia del filtro empleado se muestra en la Figura 4.3.

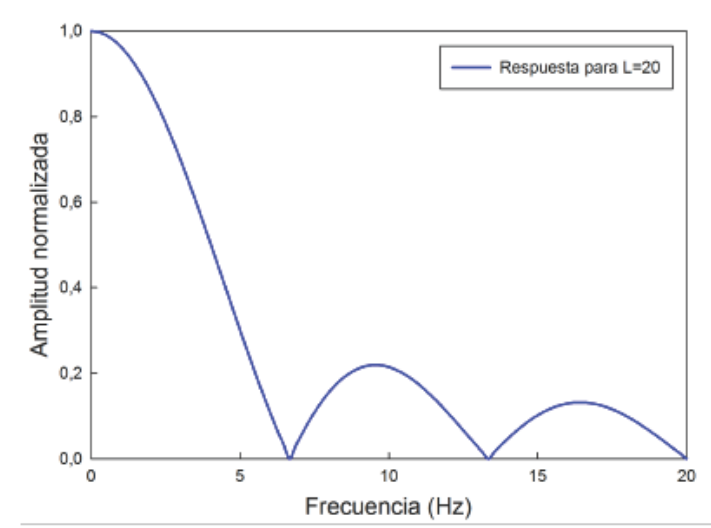

Figura 4.3: Respuesta en frecuencia del filtro de media móvil para $L=20$. 
El efecto de filtrado sobre una respuesta en frecuencia medida empleando el filtro anterior se muestra en la Figura 4.4.

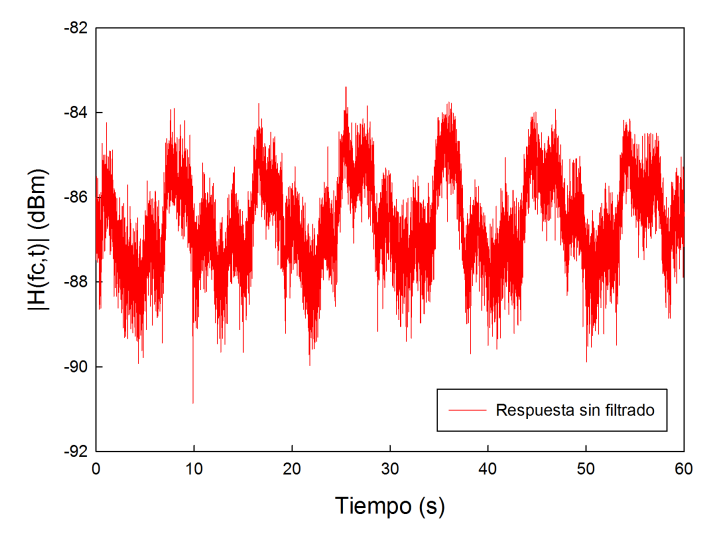

(a)

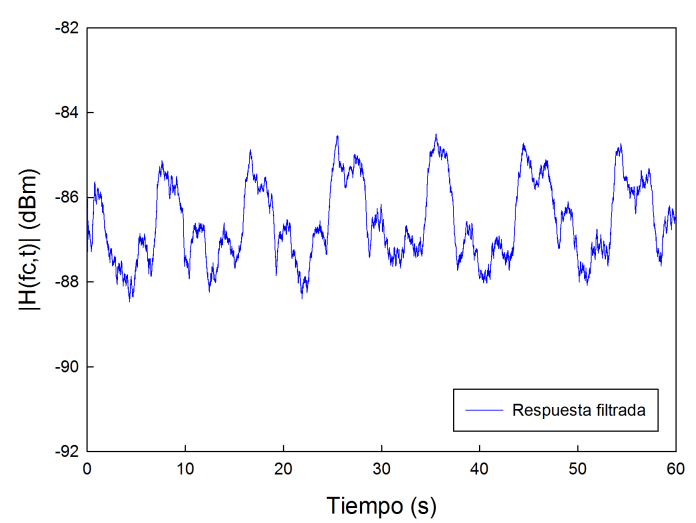

(b)

Figura 4.4: Reducción del ruido presente en la respuesta en frecuencia medida. (a) Respuesta original, (b) respuesta filtrada.

El efecto sobre el espectro Doppler $D\left(f_{c}, t\right)$ del proceso de filtrado en el tiempo de las muestras correspondientes a las respuestas $H\left(f_{c}, t\right)$ del canal medidas, es la atenuación de las componentes frecuenciales debidas a ruido fuera de la banda de paso del filtro. En la Figura 4.5 se muestra este efecto.

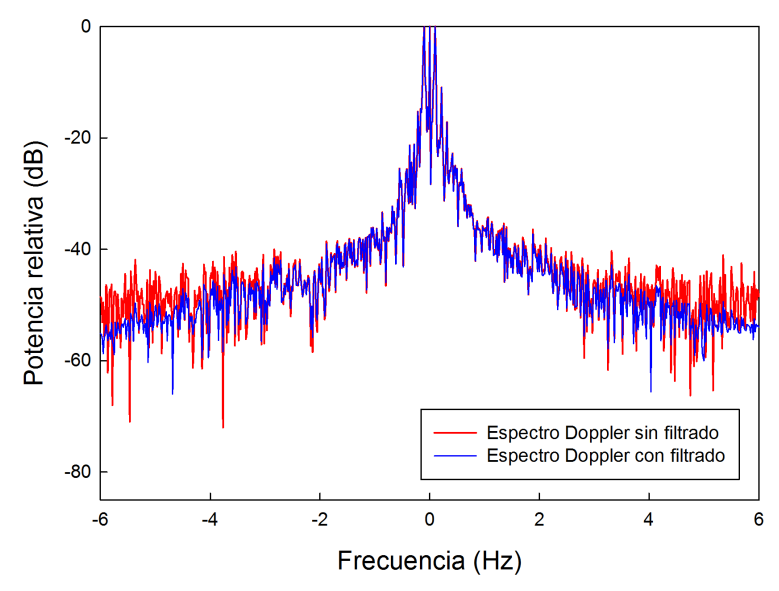

Figura 4.5: Efecto del filtrado sobre el espectro Doppler.

Como se observa en la Figura 4.5, la anchura del espectro Doppler debida a componentes con frecuencias no asociadas a ruido no se ve afectada por el filtrado empleado, mientras que las componentes a partir de los $2 \mathrm{~Hz}$ aproximadamente se ven atenuadas. 


\subsection{Efecto de la respiración en el canal in-body}

Durante la respiración el cuerpo humano obtiene oxígeno y expulsa el dióxido de carbono de su interior. En la respiración entran en juego multitud de elementos entre los que cabe destacar los pulmones, la caja torácica (compuesta básicamente por las costillas, esternón y músculos intercostales) y el diafragma. El proceso de la respiración se divide en dos fases: inspiración y espiración. Durante la inspiración, proceso de carácter activo, el diafragma se contrae realizando un movimiento hacia la cavidad abdominal con un recorrido medio de 1-2 cm en el caso de respiración tranquila, alcanzando los 10-12 cm en el caso de respiración forzada, por ejemplo durante el ejercicio. Este movimiento ejerce por un lado una compresión de los órganos situados en la parte superior del abdomen y por otro lado una variación en la dimensión vertical del muro pectoral [West, 2012]. La dimensión lateral y antero-posterior del tórax también aumenta debido a la contracción de los músculos intercostales y el movimiento de las costillas. Además, conforme los pulmones se hinchan, estos ocupan la cavidad torácica causando el desplazamiento y rotación del corazón [Shechter et al., 2004]. Durante la espiración, básicamente un proceso pasivo durante la respiración normal, los pulmones se desinflan y el muro pectoral vuelve a su posición de reposo. El ciclo de inspiración-espiración se repite entre $12-18$ veces por minuto (proceso de $0.2 \mathrm{~Hz}$ a $0.3 \mathrm{~Hz}$ ), incrementando hasta 50 veces por minuto $(0.8 \mathrm{~Hz})$ durante condiciones de respiración forzada [Garrett y Kirkendall, 1999].

En el caso de dos dispositivos de una WBAN, ubicados ambos en el interior del cuerpo (canal IB2IB) o uno de ellos en el interior y otro en la superficie del mismo (canal IB2OB), o a una cierta distancia de este (canal IB2OFF) el movimiento bien del pecho o abdomen, bien de los órganos internos durante la respiración genera un movimiento relativo entre Tx y Rx que puede ocasionar un desplazamiento en la frecuencia de la señal recibida o efecto Doppler, dando lugar a desvanecimiento temporal en el canal radio.

Con el propósito de establecer un modelo teórico que describa la variación temporal del proceso respiratorio, se han considerado las medidas previamente filtradas, empleando el filtro de media móvil de la expresión (4.1) con un tamaño de ventana $L=20$, de la respuestas al impulso variables en el tiempo $H\left(f_{c}, t\right)$ obtenidas mediante el analizador de redes vectorial, configurado este en modo $\mathrm{CW}$ con los parámetros detallados en la Tabla 4.1. Para el caso del canal IB2OB la antena transmisora se ha colocado en el interior del contenedor, inmersa en el phantom líquido y acoplada a la esfera hinchable de látex mediante adhesivo, mientras que la antena receptora se ha colocado sobre la pared exterior del contenedor, 
orientada hacia $\mathrm{Tx}$, a una distancia inicial de $13 \mathrm{~cm}$. El esquema detallado empleado para estas medidas se muestra en la Figura 4.6.

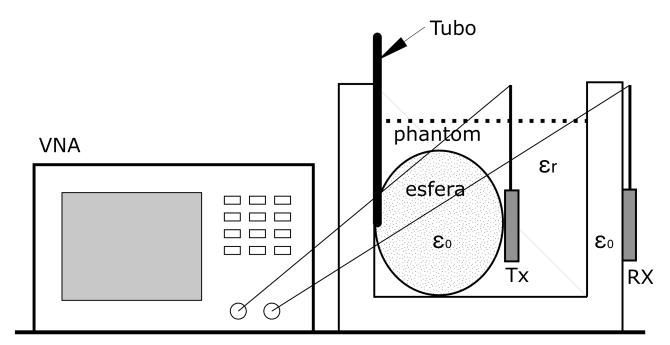

Figura 4.6: Esquema de elementos para medidas del canal dinámico IB2OB.

Para las medidas del canal IB2OFF, al igual que en el caso anterior, la antena Tx se ha colocado inmersa en el phantom pero en este caso la antena $\mathrm{Rx}$ se ha colocado sobre un soporte a cuatro distancias de separación diferentes respecto a la pared exterior del contenedor: $10 \mathrm{~cm}, 20 \mathrm{~cm}, 30 \mathrm{~cm}$ y $50 \mathrm{~cm}$, tal y como se muestra en la Figura 4.7 .

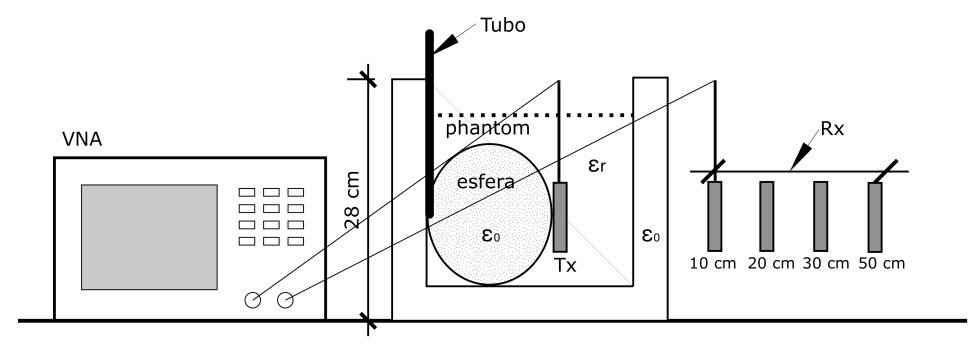

Figura 4.7: Esquema de elementos para medidas del canal dinámico IB2OFF.

Por último, para las medidas del canal IB2IB, ambas antenas se han ubicado en el interior del contenedor, inmersas en el phantom líquido guardando una separación inicial entre ellas de $7 \mathrm{~cm}$. En la Figura 4.8 se muestra el detalle de los elementos para las medidas en este canal.

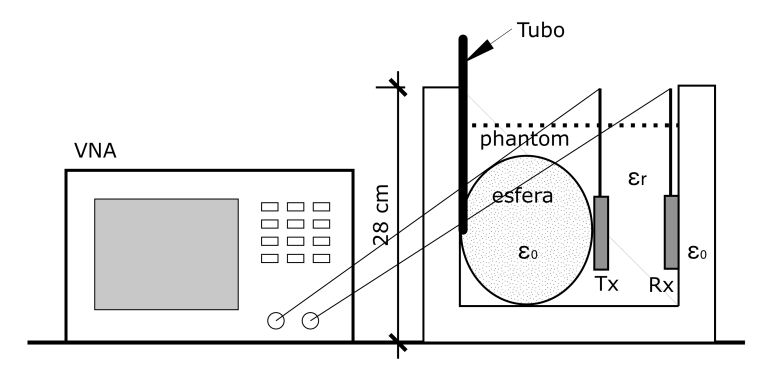

Figura 4.8: Esquema de elementos para medidas del canal dinámico IB2IB.

Para los tres escenarios anteriores la extensión del recorrido entre Tx y $\mathrm{Rx}$ se ha restringido a $4 \mathrm{~cm}$ para emular la expansión torácica humana habitual. Además, la respuesta del canal se ha medido en un intervalo de $60 \mathrm{~s}$ durante el cual, se ha insuflado/liberado de 
manera cíclica aire en el interior de la esfera de látex empleando el tubo de conexión simulando el hinchado/deshinchado de un pulmón durante las fases de inspiración y espiración.

\subsubsection{Modelado del proceso respiratorio}

Debido a la naturaleza periódica de la respiración, la variación en el tiempo de la amplitud de la señal recibida se puede considerar un proceso periódico también. Bajo condiciones ideales, esta variación presentaría un patrón sinusoidal perfecto de amplitud constante pero en la realidad, la dependencia con la frecuencia de las características dieléctricas de los tejidos biológicos hace que, conforme esta aumenta, la señal recibida presenta una mayor atenuación y distorsión. Con el fin de desarrollar un modelo teórico que describa la variación periódica en la amplitud de la señal recibida, cuantificada a partir del módulo de la respuesta al impulso variable en el tiempo $H\left(f_{c}, t\right)$ medida por medio del VNA para cada uno de los escenarios de propagación considerados, se propone la siguiente expresión,

$$
f(t)=A+\alpha \cdot \operatorname{sen}(2 \pi \beta t+\varphi)
$$

donde el parámetro $A$ representa el nivel medio de potencia de la señal recibida, el cual está relacionada con las pérdidas de propagación del canal a la frecuencia considerada $f_{c}$. El parámetro $\beta$ corresponde con la frecuencia de respiración (BR, Breathing Rate), en respiraciones por minuto. De esta forma $\beta=B R / 60$. El parámetro $\alpha$ modela la amplitud de la señal recibida y está relacionado con la máxima excursión del proceso oscilatorio entorno al valor medio de potencia de la señal recibida (parámetro $A$ ) y depende tanto de la profundidad de respiración (máxima excursión Tx-Rx) como de la respuesta en frecuencia del canal a $f_{c}$. Puede obtenerse por inspección visual como la mitad del valor pico a pico para

cada respuesta en frecuencia $H\left(f_{c}, t\right)$. Por último, $\varphi$ representa el desplazamiento en fase inicial y puede obtenerse mediante ajuste.

Respecto a la variación del parámetro $A$ del modelo con la frecuencia de transmisión, en todos los escenarios de propagación considerados (IB2OB, IB2OFF y IB2IB), para el rango de frecuencias de 3.1-8 GHz, se ha observado un comportamiento decreciente lineal $(y=a x+b)$ tal y como se muestra en la Figura 4.9. Esta variación puede relacionarse con el carácter homogéneo del phantom empleado para las medidas, por lo que en condiciones de propagación reales considerando estructuras heterogéneas compuestas por 
tejidos con diferentes propiedades dieléctricas (como son los órganos humanos), la variación puede no ser lineal.

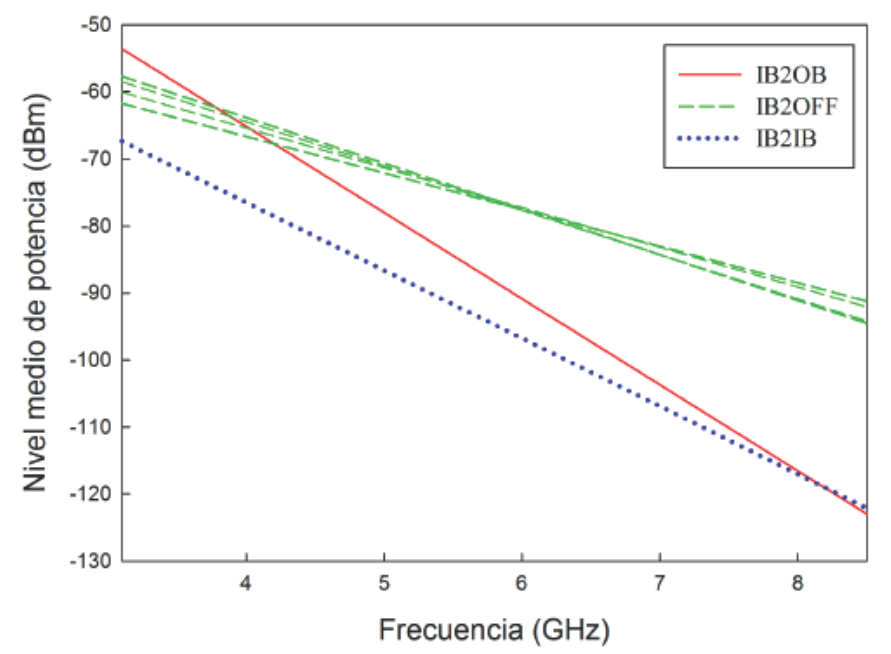

Figura 4.9: Variación con la frecuencia de la potencia media recibida (parámetro A) para IB2IB, IB2OB y IB2OFF $(10 \mathrm{~cm}, 20 \mathrm{~cm}, 30 \mathrm{~cm}$ y $50 \mathrm{~cm})$.

En la Figura 4.9 se observa que, para el grupo de medidas en el canal IB2OFF la variación con la frecuencia de la potencia recibida en las cuatro distancias de separación consideradas es similar, con un valor medio de la pendiente de -6.21. Esto puede deberse a que las pérdidas en el interior del phantom son muy superiores a las correspondientes a la propagación en el aire. Como consecuencia, la variación en la distancia off-body $(10-50 \mathrm{~cm})$ no afecta de manera significativa en el nivel de potencia recibida. Para el caso de las medidas del canal IB2IB, la pendiente obtenida es de -10.14 , mientras que el canal IB2OB presenta la mayor pendiente de los tres casos, con un valor de -12.85 .

Con el fin de evaluar la bondad de ajuste del modelo propuesto sobre los valores medidos correspondientes al modulo de la señal recibida en cada escenario de propagación, se ha empleado como criterio la raíz cuadrada del error cuadrático medio (RMSE) definido como,

$$
R M S E=\sqrt{\frac{1}{n} \sum_{i=1}^{n}\left(X_{i}-\bar{X}_{i}\right)^{2}}
$$

donde $X_{i}-\bar{X}_{i}$ es la diferencia entre la i-ésima muestra del módulo de la respuesta en frecuencia medida para el canal considerado y la del modelo teórico de la expresión (4.3). Esta medida da una idea la desviación típica del error de ajuste, de forma que conforme este se acerca a 0 , el modelo propuesto se ajusta de manera más perfecta al proceso original medido. 
Debido a que el proceso real tiene una duración de 60 segundo y durante este tiempo no todos los periodos presentan un patrón periódico similar (por variaciones en la excursión máxima Tx-Rx, distorsión debida a la frecuencia, etc.), la bondad de ajuste se ha evaluado considerando únicamente los tres periodos consecutivos del proceso más estables, seleccionados de forma visual. Ya que los valores correspondientes a los parámetros $A, \alpha$ y $\beta$ pueden obtenerse directamente del proceso real, el único parámetro que resta obtener es $\varphi$, por lo que la obtención de este y el cálculo del RMSE se ha realizado mediante ajuste con Matlab y la herramienta cftool.

A continuación, se presentan detallados para cada uno de los escenarios de propagación considerados, teniendo en cuenta la variación sobre el canal radio debida a la respiración, los parámetros de ajuste sobre el modelo propuesto.

\subsubsection{Modelado del proceso respiratorio para el canal IB2OB}

En la Figura 4.10 se muestra para cada frecuencia considerada el ajuste por medio del modelo teórico definido en la expresión (4.3) del módulo de las respuestas en frecuencia variables en el tiempo $H\left(f_{c}, t\right)$ medidas para el canal IB2OB.

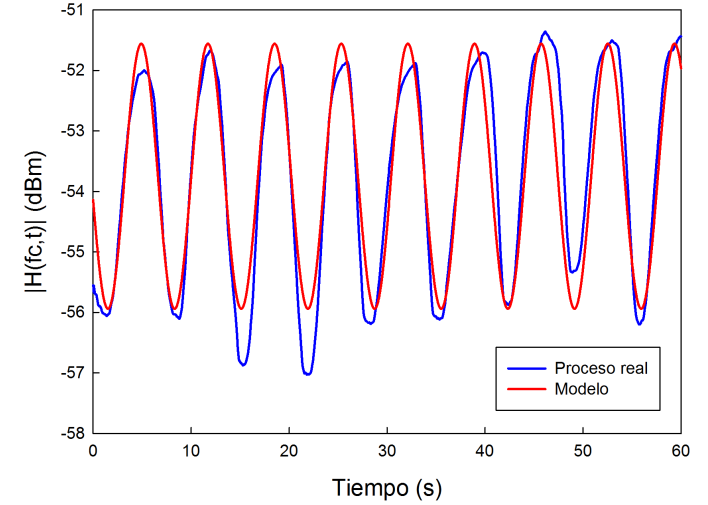

(a)

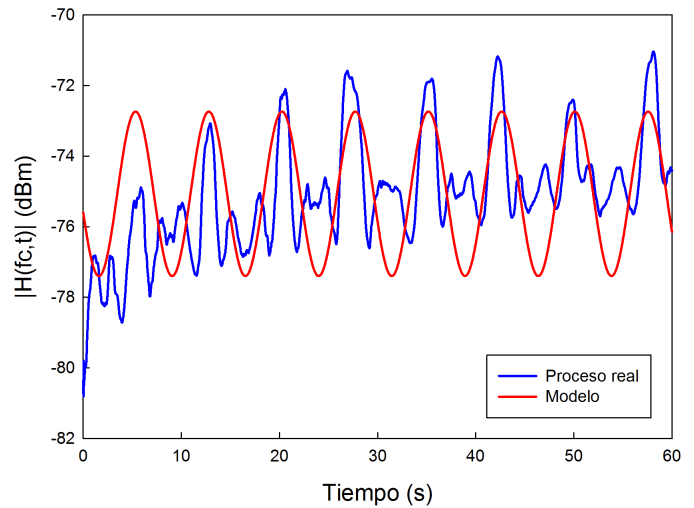

(b) 


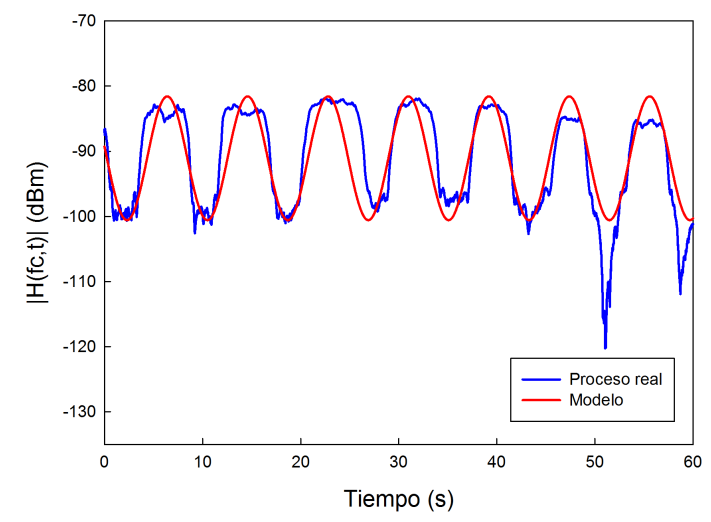

(c)

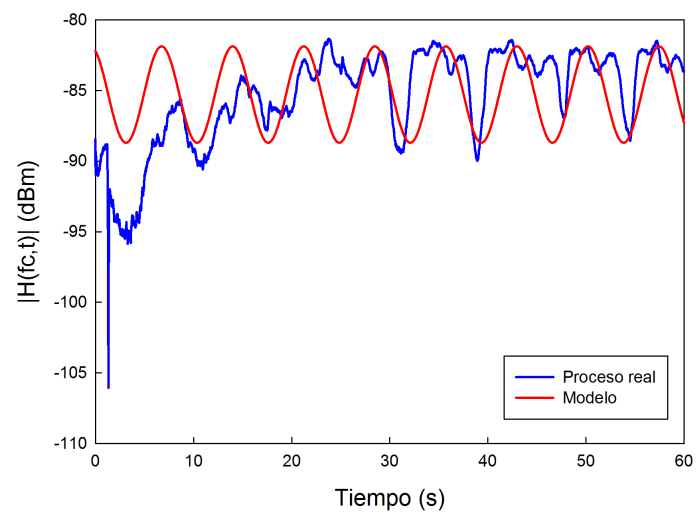

(d)

Figura 4.10: Ajuste de $\left|H\left(f_{c}, t\right)\right|$ al modelo teórico para el canal IB2OB. (a) $3.1 \mathrm{GHz}$, (b) $4.8 \mathrm{GHz}$, (c) $6 \mathrm{GHz}$ y (d) $8.5 \mathrm{GHz}$.

Los parámetros correspondientes al ajuste sobre el modelo teórico así como el valor del criterio RMSE para cada frecuencia considerada se detallan en la Tabla 4.2.

\begin{tabular}{|c|c|c|c|c|c|}
\hline $\begin{array}{c}\boldsymbol{f}_{\boldsymbol{c}} \\
(\mathbf{G H z})\end{array}$ & $\boldsymbol{A}$ & $\boldsymbol{\alpha}$ & $\boldsymbol{\beta}$ & $\boldsymbol{\varphi}$ & RMSE \\
\hline $\mathbf{3 . 1}$ & -53.75 & 2.19 & 0.147 & 3.19 & 0.60 \\
\hline $\mathbf{4 . 8}$ & -75.07 & 2.33 & 0.134 & 3.48 & 1.46 \\
\hline $\mathbf{6}$ & -91.08 & 9.49 & 0.122 & 2.72 & 2.67 \\
\hline $\mathbf{8 . 5}$ & -85.29 & 3.41 & 0.138 & 2.53 & $2.88^{*}$ \\
\hline
\end{tabular}

Tabla 4.2: Valores del modelo teórico de proceso respiratorio para el canal IB2OB.

Tal y como se observa en la Figura 4.10, conforme aumenta la frecuencia la amplitud de la señal recibida presenta un mayor nivel de distorsión, lo cual hace que el ajuste del modelo teórico sobre el proceso real sea cada vez peor. A $3.1 \mathrm{GHz}$ el ajuste es bueno (RMSE $=0.60$ ) empeorando hasta los $8.5 \mathrm{GHz}$ en donde el modelo obtenido, a pesar de obtener un valor para $\varphi$ y el RMSE mediante Matlab, el ajuste del proceso original no es posible empleando el modelo propuesto. Esto se ha identificado con un asterisco (*) en la tabla anterior. Además, se puede observar que a $4.8 \mathrm{GHz}$ la amplitud de la respuesta en frecuencia medida presenta un nivel de distorsión superior al que se da para $6 \mathrm{GHz}$. Esto puede estar relacionado tanto con la variación en el patrón de radiación, como con la respuesta en frecuencia en campo cercano de las antenas empleadas para esa frecuencia. 


\subsubsection{Modelado del proceso respiratorio para el canal IB2OFF}

En la Figura 4.11 y Figura 4.12 se muestran para cada frecuencia considerada el ajuste por medio del modelo teórico definido en la expresión (4.3) del módulo de las respuestas en frecuencia variables en el tiempo $H\left(f_{c}, t\right)$ medidas para el canal IB2OFF a las distancias de separación entre Tx y Rx de $10 \mathrm{~cm}$ y $50 \mathrm{~cm}$.

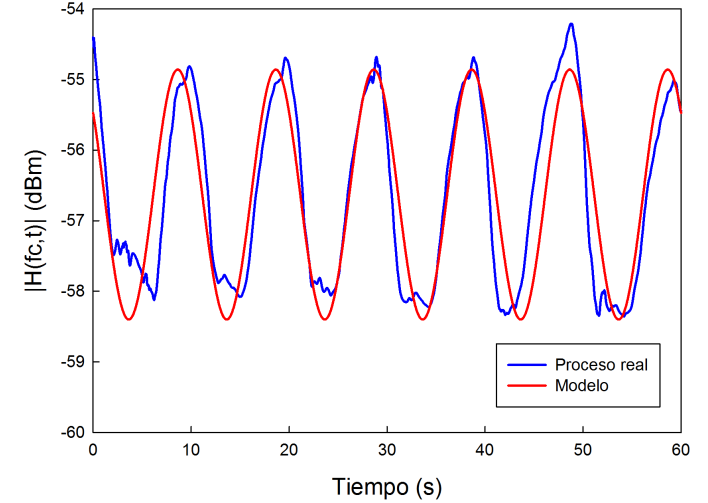

(a)

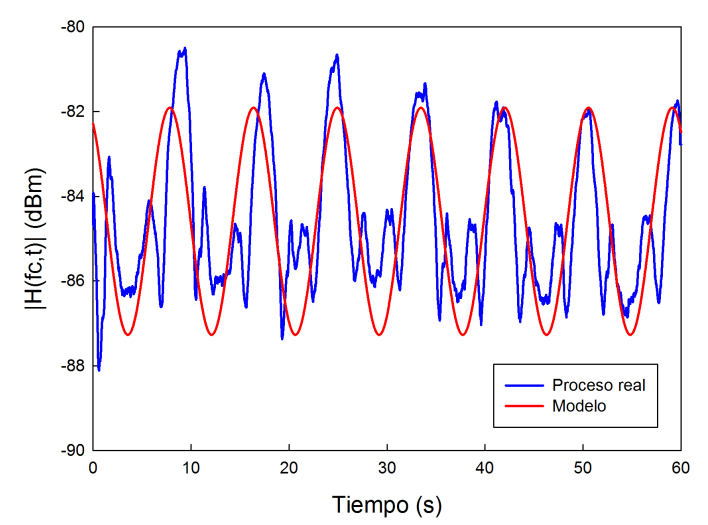

(c)

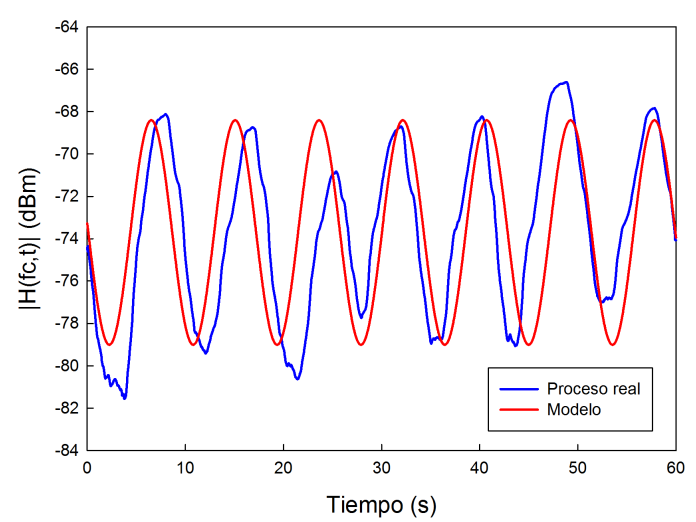

(b)

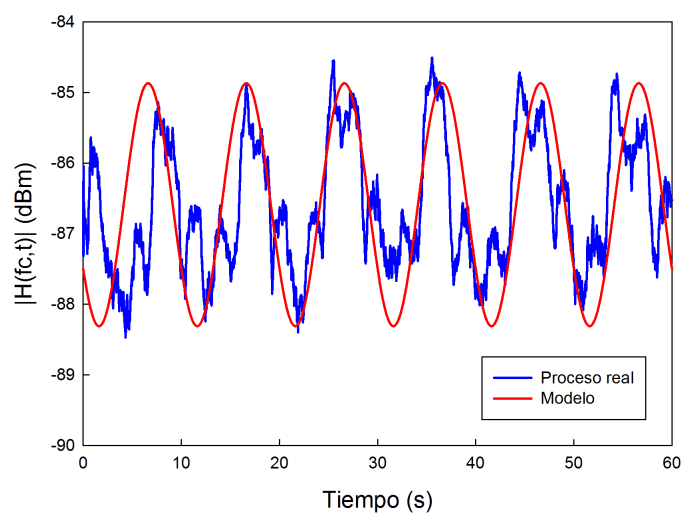

(d)

Figura 4.11: Ajuste de $H\left(f_{c}, t\right)$ al modelo teórico para el canal IB2OFF para $10 \mathrm{~cm}$ entre Tx y Rx. (a) $3.1 \mathrm{GHz}$, (b) $4.8 \mathrm{GHz}$, (c) $6 \mathrm{GHz}$ y (d) $8.5 \mathrm{GHz}$.

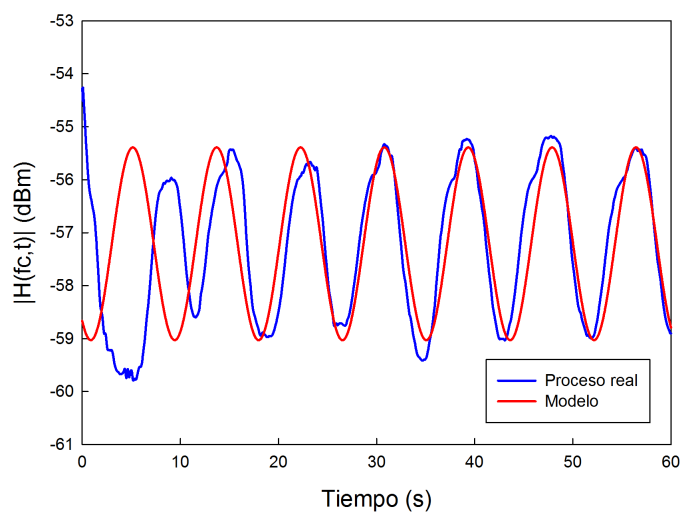

(a)

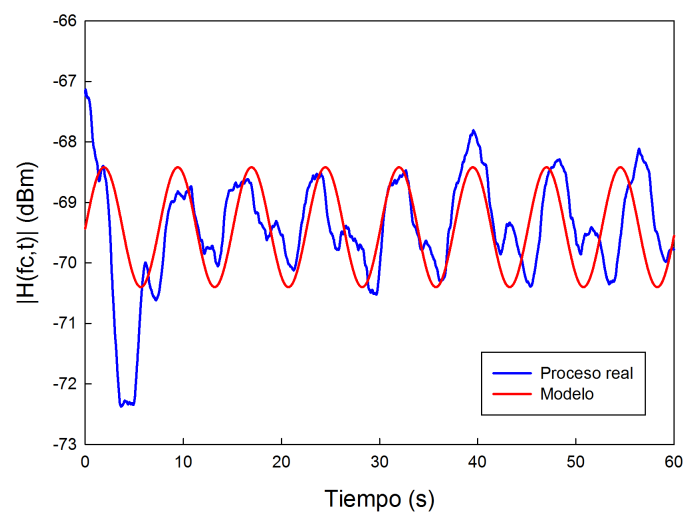

(b) 


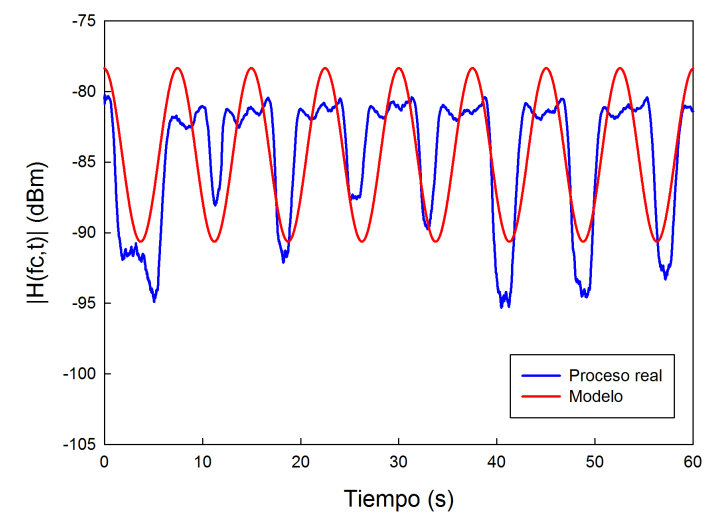

(c)

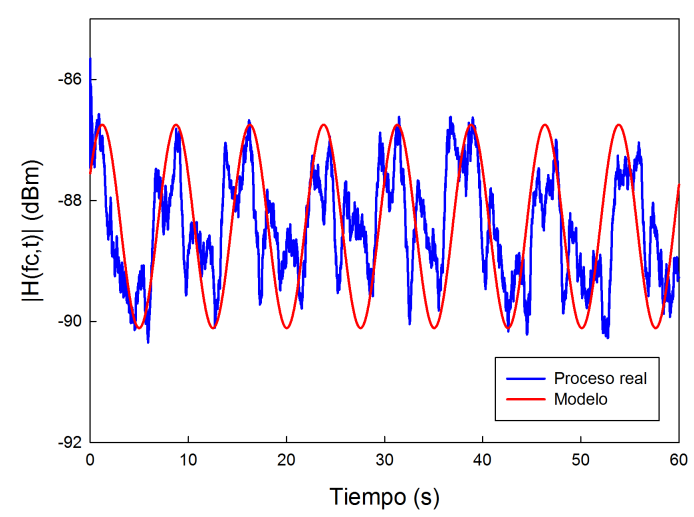

(d)

Figura 4.12: Ajuste de $H\left(f_{c}, t\right)$ al modelo teórico para el canal IB2OFF para $50 \mathrm{~cm}$ entre Tx y Rx. (a) $3.1 \mathrm{GHz}$, (b) $4.8 \mathrm{GHz}$, (c) $6 \mathrm{GHz}$ y (d) $8.5 \mathrm{GHz}$.

Los parámetros correspondientes al ajuste sobre el modelo teórico así como el valor del criterio RMSE para cada frecuencia considerada y distancia de separación off-body se detallan en la Tabla 4.3.

\begin{tabular}{|c|c|c|c|c|c|c|}
\hline $\begin{array}{c}\text { Distancia } \\
\text { Off-body } \\
(\mathbf{c m})\end{array}$ & $\begin{array}{c}\boldsymbol{f}_{\boldsymbol{c}} \\
(\mathbf{G H z})\end{array}$ & $\boldsymbol{A}$ & $\boldsymbol{\alpha}$ & $\boldsymbol{\beta}$ & $\boldsymbol{\varphi}$ & RMSE \\
\hline \multirow{4}{*}{$\mathbf{1 0}$} & $\mathbf{3 . 1}$ & -56.63 & 1.77 & 0.100 & 2.51 & 0.46 \\
\cline { 2 - 7 } & $\mathbf{4 . 8}$ & -73.71 & 5.3 & 0.117 & -2.52 & 1.65 \\
\cline { 2 - 7 } & $\mathbf{6}$ & -84.59 & 2.68 & 0.117 & 2.39 & 1.27 \\
\cline { 2 - 7 } & $\mathbf{8 . 5}$ & -86.59 & 1.72 & 0.100 & -2.29 & 0.68 \\
\hline \multirow{4}{*}{$\mathbf{2 0}$} & $\mathbf{3 . 1}$ & -54.73 & 0.67 & 0.100 & -2.19 & 0.24 \\
\cline { 2 - 7 } & $\mathbf{4 . 8}$ & -68.49 & 0.77 & 0.117 & 1.35 & 0.37 \\
\cline { 2 - 7 } & $\mathbf{6}$ & -84.97 & 6.43 & 0.117 & -1.29 & 1.49 \\
\cline { 2 - 7 } & $\mathbf{8 . 5}$ & -90.69 & 2.01 & 0.117 & 2.44 & 1.0 \\
\hline \multirow{4}{*}{$\mathbf{3 0}$} & $\mathbf{3 . 1}$ & -54.13 & 0.79 & 0.100 & 2.99 & 0.39 \\
\cline { 2 - 7 } & $\mathbf{4 . 8}$ & -70.99 & 1.59 & 0.117 & 2.29 & $1.13 *$ \\
\cline { 2 - 7 } & $\mathbf{6}$ & -85.34 & 2.89 & 0.117 & -1.89 & $2.57 *$ \\
\cline { 2 - 7 } & $\mathbf{8 . 5}$ & -89.74 & 2.57 & 0.133 & 1.71 & 1.07 \\
\hline \multirow{4}{*}{$\mathbf{5 0}$} & $\mathbf{3 . 1}$ & -57.21 & 1.82 & 0.117 & -1.88 & 0.34 \\
\cline { 2 - 7 } & $\mathbf{4 . 8}$ & -69.41 & 0.99 & 0.133 & -0.39 & 0.50 \\
\cline { 2 - 7 } & $\mathbf{6}$ & -83.30 & 3.38 & 0.133 & 1.97 & 2.07 \\
\cline { 2 - 7 } & $\mathbf{8 . 5}$ & -88.43 & 1.68 & 0.133 & 0.89 & $0.94 *$ \\
\hline
\end{tabular}

Tabla 4.3: Valores del modelo teórico de proceso respiratorio para el canal IB2OFF.

Respecto a la variación con la frecuencia de la bondad de ajuste del modelo sobre el proceso real, tal y como se puede observar a partir de $4.8 \mathrm{GHz}$, esta no sigue una tendencia clara conforme aumenta la frecuencia de operación. En la Tabla 4.3 se han marcado con un asterisco aquellos casos para los cuales, a pesar de obtener un valor de $\varphi$ y RMSE, el modelo teórico no se ajusta al proceso real. 
Tal y como se observa tanto en las Figuras 4.11 y 4.12 como en la Tabla 4.3, la variación del valor para el parámetro $A$ correspondiente al nivel medio de potencia recibido presenta una dependencia con la frecuencia mayor que con la distancia entre Tx y Rx. Esta variación se muestra en la Figura 4.13.

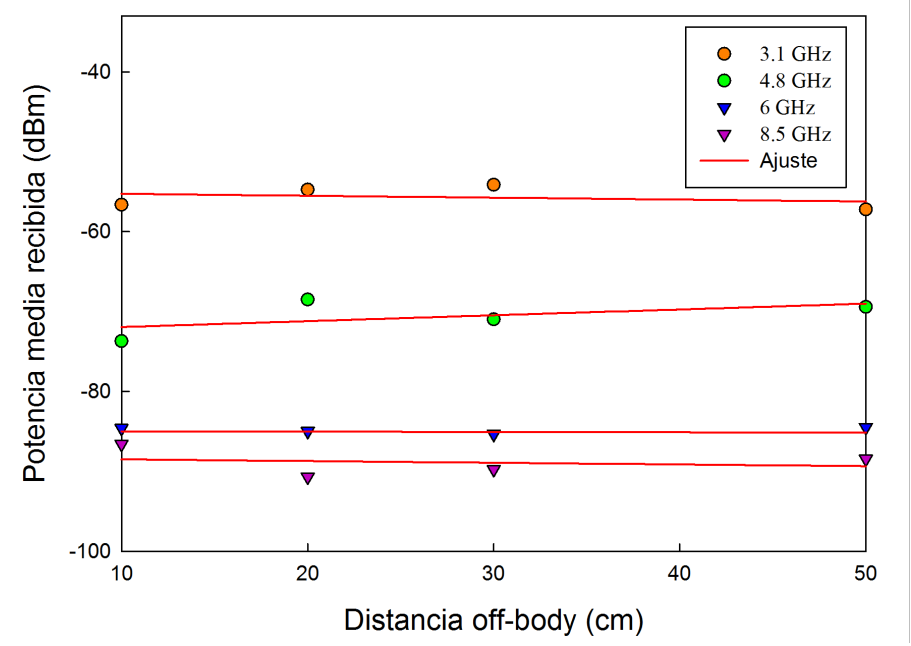

Figura 4.13: Variación en la potencia media recibida (parámetro A) según distancia para el canal IB2OFF.

Los valores absolutos medidos para las pendientes, a cada frecuencia considerada según la distancia off-body son $0.024,0.074,0.0037$ y 0.021 , con unos valores de desviación típica $\sigma$ de $1.42,1.91,0.40$ y 1.78 respectivamente para los valores de frecuencia $f_{c}$ de 3.1 GHz, $4.8 \mathrm{GHz}, 6 \mathrm{GHz}$ y $8.5 \mathrm{GHz}$. Esto demuestra que la variación en la potencia media recibida con la distancia, para el conjunto de separaciones considerado es despreciable frente a la variación con la frecuencia.

El parámetro $\alpha$ no se observa que presente una variación clara con la distancia de separación off-body. Para la frecuencia de $8.5 \mathrm{GHz}$, este parámetro presenta una desviación típica de $0.39 \mathrm{~dB}$ (comparado con los valores de $1.01 \mathrm{~dB}, 1.9 \mathrm{~dB}$ y $2.13 \mathrm{~dB}$ para el resto de frecuencias). La razón de esta variación puede estar debida a un efecto de saturación producido por la cercanía de los valores medidos con el nivel de ruido del sistema.

\subsubsection{Modelado del proceso respiratorio para el canal IB2IB}

En la Figura 4.14 se muestra para cada frecuencia considerada el ajuste por medio del modelo teórico definido en la expresión (4.3) del módulo de las respuestas en frecuencia variables en el tiempo $H\left(f_{c}, t\right)$ medidas para el canal IB2IB. 


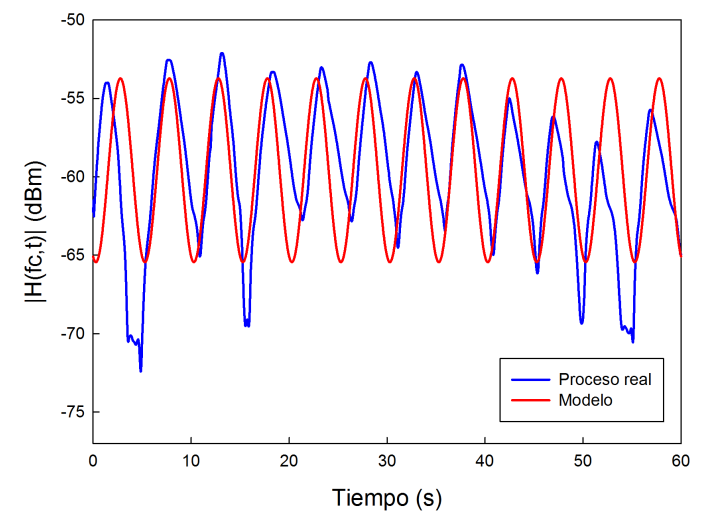

(a)

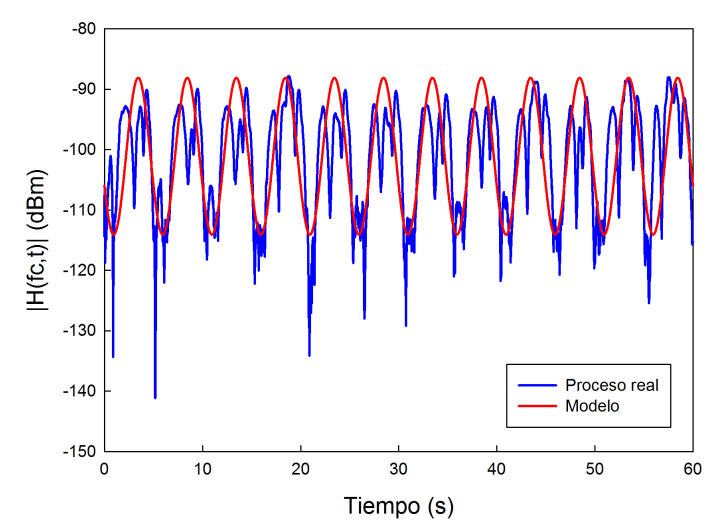

(c)

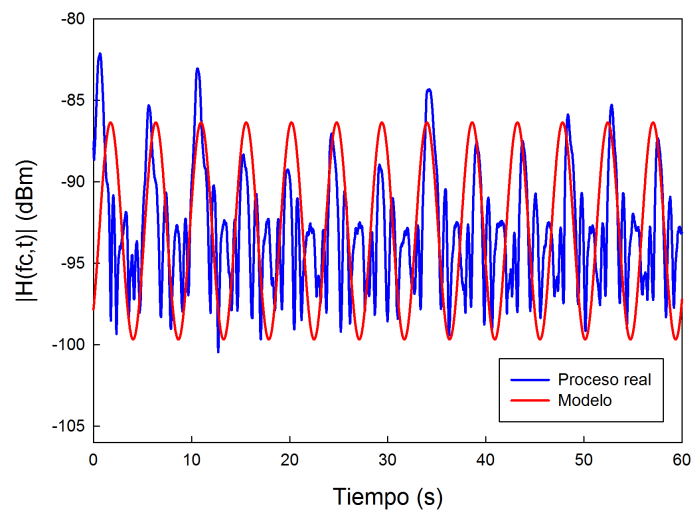

(b)

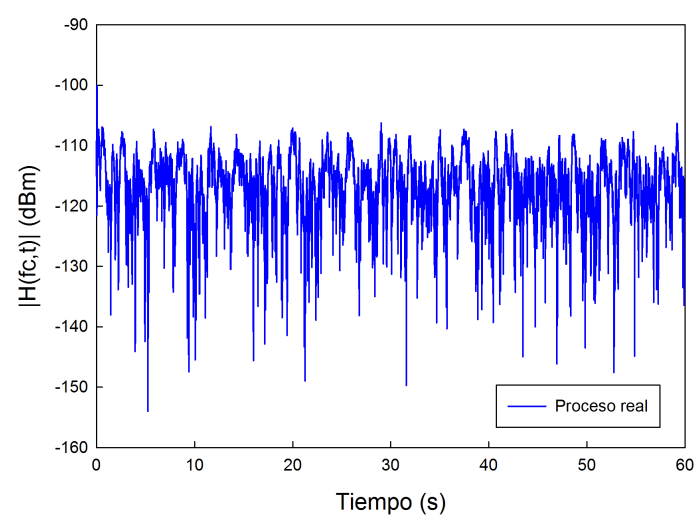

(d)

Figura 4.14: Ajuste de $H\left(f_{c}, t\right)$ al modelo teórico para el canal IB2IB. (a) $3.1 \mathrm{GHz}$, (b) $4.8 \mathrm{GHz}$, (c) 6 GHz y (d) $8.5 \mathrm{GHz}$.

Los parámetros correspondientes al ajuste sobre el modelo teórico así como el valor del criterio RMSE para cada frecuencia considerada se detallan en la Tabla 4.4.

\begin{tabular}{|c|c|c|c|c|c|}
\hline $\begin{array}{c}\boldsymbol{f}_{\boldsymbol{c}} \\
(\mathbf{G H z})\end{array}$ & $\boldsymbol{A}$ & $\boldsymbol{\alpha}$ & $\boldsymbol{\beta}$ & $\boldsymbol{\varphi}$ & RMSE \\
\hline $\mathbf{3 . 1}$ & -59.59 & 5.86 & 0.200 & -2.80 & 1.97 \\
\hline $\mathbf{4 . 8}$ & -93.02 & 6.66 & 0.217 & -0.43 & 3.70 \\
\hline $\mathbf{6}$ & -101.1 & 12.97 & 0.200 & 3.28 & 7.1 \\
\hline $\mathbf{8 . 5}$ & - & - & - & - & - \\
\hline
\end{tabular}

Tabla 4.4: Valores del modelo teórico de proceso respiratorio para el canal IB2IB.

Respecto al ajuste del modelo teórico sobre el proceso real, al igual que se observó para el caso del canal IB2OB, conforme aumenta la frecuencia el valor del RMSE es mayor. Esto se puede observar en la Figura 4.14. Para el caso de $8.5 \mathrm{GHz}$ no es posible derivar los parámetros del modelo al estar el nivel de potencia de la señal recibida bajo el umbral de ruido del sistema. 
Para el caso del canal de propagación IB2IB, únicamente la frecuencia de $3.1 \mathrm{GHz}$ puede considerarse operativa, ya que para el resto de frecuencias consideradas, los niveles de potencia media recibida se encuentran por bajo del nivel de ruido del sistema (para $8.5 \mathrm{GHz}$ ) o muy cerca del mismo (para $4.8 \mathrm{GHz}$ y $6 \mathrm{GHz}$ ). Esto puede ser debido a la dependencia con la frecuencia de la interacción en campo de cercano de ambas antenas separadas $7 \mathrm{~cm}$.

\subsection{Caracterización en frecuencia del canal in-body dinámico}

El estudio del efecto en frecuencia causado por el movimiento entre dos antenas (Tx y Rx), considerando al menos una de ellas ubicada en el interior del cuerpo, se ha realizado por medio de la caracterización del efecto Doppler considerando por un lado, el análisis de la forma del mismo y por otro lado el ensanchamiento en frecuencia cuantificado a partir de los parámetros Doppler spread $B_{D}$ y rms Doppler spread $\rho_{r m s}$. Para ello se han calculado mediante la expresión (A.16) los espectros Doppler $D\left(f_{c}, \rho\right)$ correspondientes a cada una de las medidas, previamente filtradas empleando el filtro de media móvil de la expresión (4.1) con un tamaño de ventana $L=20$, de las respuestas al impulso variables en el tiempo $H\left(f_{c}, t\right)$ obtenidas mediante el analizador de redes vectorial, configurado este en modo $\mathrm{CW}$ con los parámetros detallados en la Tabla 4.1. Para el caso del canal IB2OB la antena transmisora se ha colocado en el interior del contenedor, inmersa en el phantom líquido y acoplada a la esfera hinchable de látex mediante adhesivo, mientras que la antena receptora se ha colocado sobre la pared exterior del contenedor, orientada hacia Tx, a una distancia inicial de $13 \mathrm{~cm}$, tal y como se detalla en la Figura 4.6. Para las medidas del canal IB2OFF, al igual que en el caso anterior, la antena Tx se ha colocado inmersa en el phantom pero en este caso la antena $\mathrm{Rx}$ se ha colocado sobre un soporte a cuatro distancias de separación diferentes respecto al contenedor: $10 \mathrm{~cm}, 20 \mathrm{~cm}, 30 \mathrm{~cm} \mathrm{y} 50 \mathrm{~cm}$, tal y como se detalla en la Figura 4.7. Por último, para las medidas del canal IB2IB, ambas antenas se han ubicado en el interior del contenedor, inmersas en el phantom líquido guardando una separación inicial entre ellas de $7 \mathrm{~cm}$. El esquema detallado de los elementos para estas medidas se muestra en la Figura 4.8 .

Para los tres escenarios anteriores la extensión del recorrido entre Tx y Rx se ha restringido a $4 \mathrm{~cm}$ para emular la expansión torácica humana habitual. Además, la respuesta del canal se ha medido en un intervalo de $60 \mathrm{~s}$ durante el cual, se ha insuflado/liberado de manera cíclica aire en el interior de la esfera de látex empleando el tubo de conexión simulando el hinchado/deshinchado de un pulmón durante las fases de inspiración y espiración. 


\subsubsection{Modelado de la forma del espectro Doppler}

Debido a que la recepción de componentes multicamino no está angularmente equiespaciada entre $[-\pi, \pi]$ y existe una componente dominante debida a visión directa (LoS) entre Tx y Rx, la forma del espectro Doppler $D\left(f_{c} \rho\right)$ va a diferir de la clásica forma de U prevista por el modelo de Jakes [Rappaport, 1996]. Por el contrario, presentará una forma aproximadamente simétrica respecto a $0 \mathrm{~Hz}$ con un máximo a esta frecuencia debido a la componente LOS y con máximos secundarios a menor potencia a la frecuencia del proceso respiratorio y sus sucesivos armónicos, mezclados con componentes debidas al ruido. La componente de continua (dc) a $0 \mathrm{~Hz}$ se ha observado que presenta una amplitud aleatoria respecto al nivel de la primera componente frecuencial significativa. Esto puede provocar errores en la estima de los parámetros de ensanchamiento Doppler si, como en el caso actual, se establece un nivel fijo de umbral bajo la componente principal. Por este motivo se ha realizado un proceso de normalización en la amplitud de cada espectro consistente en primer lugar, en reducir la potencia de la componente a $0 \mathrm{~Hz}$ e igualarla a la potencia correspondiente a la primera componente frecuencial significativa con mayor nivel de potencia. Tras esto, el espectro se ha normalizado para que la componente a $0 \mathrm{~Hz}$ tenga un nivel de potencia de $0 \mathrm{~dB}$. En la Figura 4.15 se muestra el resultado del anterior proceso de normalización del espectro Doppler.

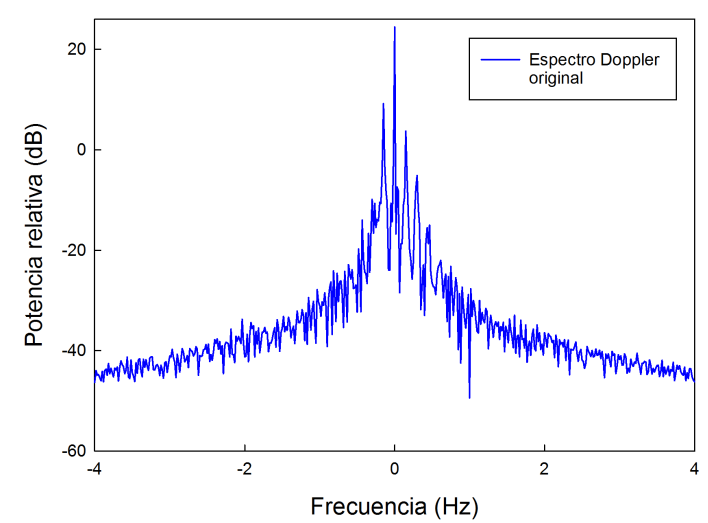

(a)

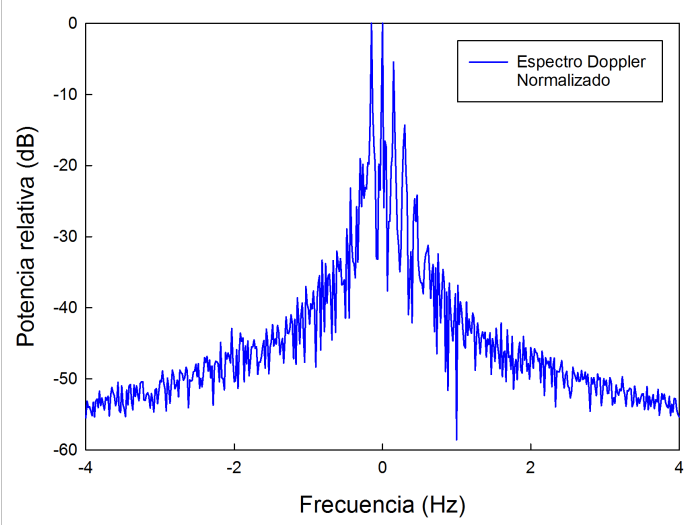

(b)

Figura 4.15: Normalización en amplitud del espectro Doppler. (a) Espectro sin normalizar, (b) espectro normalizado a $0 \mathrm{~dB}$.

De manera general, si no hay contribuciones adicionales debidas a la interacción con el entorno (condiciones de propagación en cámara anecoica), la forma del espectro Doppler se puede modelar según [Liu et al., 2013] a partir de la siguiente expresión, 


$$
f(\rho)=\frac{1}{1+2 \rho^{2}}+\sum_{m \neq 0}^{\infty} \frac{1}{m a+s\left(\rho-m f_{0}\right)^{2}} \frac{1}{x b^{(|m|-1)}}
$$

donde $f_{0}$ es la frecuencia fundamental, $m$ es el orden de los armónicos, $x$ y $b$ describen el decaimiento global y local del espectro a la frecuencia de los armónicos, y a describe el ensanchamiento Doppler entorno a los armónicos. Este modelo ha sido empleado en [Petrillo et al., 2015] para modelar el espectro Doppler debido a la respiración en el canal on-body a $60 \mathrm{GHz}$ en cámara anecoica. Pero tal y como se indica en el estudio original, debido a la complejidad de su aplicación en entornos donde es difícil determinar los armónicos presentes en el espectro Doppler (como en entornos indoor), se puede emplear la siguiente expresión simplificada,

$$
f(\rho)=\frac{1}{1+s \cdot \rho \mid}
$$

Aunque esta expresión modela de manera precisa la forma del espectro Doppler en general, cuando interesa únicamente caracterizar la forma de este en un intervalo pequeño de frecuencias, como es el caso actual (aproximadamente [-4 Hz, $4 \mathrm{~Hz}]$ ) la expresión anterior se ha observado que no proporciona el ajuste más óptimo. Con el fin de modelar la forma del espectro Doppler dentro del rango de frecuencias de interés, se ha realizado un proceso de ajuste de esta sobre tres funciones de distribución que han sido consideradas por otros autores en diferentes estudios: normal o gaussiana [D’Errico y Ouvry, 2011], [Fu et al., 2011] y [Hanssens et al., 2016], Cauchy [Hanssens et al., 2016] y Laplace [D’Errico y Ouvry, 2011], [Fu et al., 2011] y [Hanssens et al., 2016]. Las expresiones para cada una de las funciones de distribución consideradas son las siguientes:

- Normal o gaussiana:

$$
f(x)=\frac{1}{\sqrt{2 \pi \sigma^{2}}} e^{\frac{-(x-\mu)^{2}}{2 \sigma^{2}}}
$$

donde $\mu$ y $\sigma$ son respectivamente la media y la deviación típica de la función. 
- Cauchy:

$$
f(x)=\left(\frac{\lambda}{\pi}\right) \frac{1}{\lambda^{2}+(x-\alpha)^{2}}
$$

donde $\lambda$ es un parámetro de escala de la distribución e indica el ancho medio al máximo medio, y $\alpha$ es la posición del máximo de la función.

- Laplace:

$$
f(x)=\frac{1}{2 s} e^{-\frac{|x-\alpha|}{s}}
$$

donde $\alpha$ es la posición del máximo de la función y $s$ es un factor de escala.

Las funciones anteriores se han elegido por ser simétricas y continuas en el rango $x \in(-\infty,+\infty)$. Para evaluar la bondad en el ajuste a cada una de las funciones de distribución anteriores sobre la forma del espectro Doppler se ha empleado como criterio la minimización de la raíz cuadrada del error cuadrático medio (RMSE), definido por medio de la expresión (4.4).

\subsubsection{Canal de propagación IB2OB}

En la Tabla 4.5 se describe para cada una de las funciones de ajuste consideradas, los valores del criterio RMSE para cada una de las frecuencias de operación en el canal de propagación IB2OB.

\begin{tabular}{|c|c|c|c|}
\hline $\begin{array}{c}\boldsymbol{f}_{\boldsymbol{c}} \\
(\mathbf{G H z})\end{array}$ & Normal & Cauchy & Laplace \\
\hline $\mathbf{3 . 1}$ & 0.072 & 0.066 & 0.063 \\
\hline $\mathbf{4 . 8}$ & 0.082 & 0.086 & 0.081 \\
\hline $\mathbf{6}$ & 0.074 & 0.071 & 0.069 \\
\hline $\mathbf{8 . 5}$ & 0.082 & 0.078 & 0.076 \\
\hline
\end{tabular}

Tabla 4.5: Valor de RMSE para ajuste de la forma del espectro Doppler en el canal IB2OB.

Tal y como se observa en la Tabla 4.5, la función que mejor se ajusta a la forma del espectro Doppler para todas las frecuencias en el canal IB2OB es la de Laplace, siendo la normal la que, en general, presenta un peor ajuste. En la Figura 4.16 se muestran las funciones de ajuste para el caso de $f_{c}=3.1 \mathrm{GHz}$. 


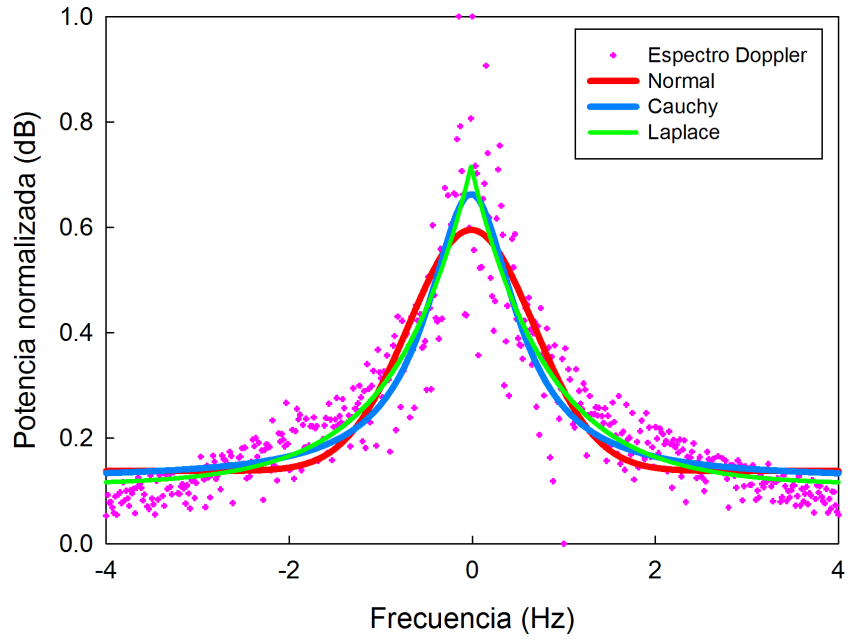

Figura 4.16: Ajuste de la forma del espectro Doppler para el canal IB2OB a 3.1 GHz.

\subsubsection{Canal de propagación IB2OFF}

En la Tabla 4.6 se detalla, al igual que en el caso del canal IB2OB, para cada una de las funciones de ajuste consideradas, los valores del criterio RMSE para cada una de las frecuencias de operación en el canal de propagación IB2OB a las cuatro distancias de separación: $10 \mathrm{~cm}, 20 \mathrm{~cm}, 30 \mathrm{~cm}$ y $50 \mathrm{~cm}$.

\begin{tabular}{|c|c|c|c|c|}
\hline $\begin{array}{c}\text { Distancia } \\
\text { Tx-Rx } \\
(\mathbf{c m})\end{array}$ & $\begin{array}{c}\boldsymbol{f}_{\boldsymbol{c}} \\
(\mathbf{G H z})\end{array}$ & Normal & Cauchy & Laplace \\
\hline \multirow{4}{*}{$\mathbf{1 0}$} & $\mathbf{3 . 1}$ & 0.073 & 0.063 & 0.061 \\
\cline { 2 - 5 } & $\mathbf{4 . 8}$ & 0.073 & 0.068 & 0.064 \\
\cline { 2 - 5 } & $\mathbf{6}$ & 0.078 & 0.084 & 0.079 \\
\cline { 2 - 5 } & $\mathbf{8 . 5}$ & 0.076 & 0.069 & 0.065 \\
\hline \multirow{4}{*}{$\mathbf{2 0}$} & $\mathbf{3 . 1}$ & 0.076 & 0.065 & 0.059 \\
\cline { 2 - 5 } & $\mathbf{4 . 8}$ & 0.076 & 0.067 & 0.059 \\
\cline { 2 - 5 } & $\mathbf{6}$ & 0.074 & 0.077 & 0.072 \\
\cline { 2 - 5 } & $\mathbf{8 . 5}$ & 0.079 & 0.075 & 0.074 \\
\hline \multirow{4}{*}{30} & $\mathbf{3 . 1}$ & 0.073 & 0.063 & 0.055 \\
\cline { 2 - 5 } & $\mathbf{4 . 8}$ & 0.072 & 0.072 & 0.065 \\
\cline { 2 - 5 } & $\mathbf{6}$ & 0.075 & 0.081 & 0.074 \\
\cline { 2 - 5 } & $\mathbf{8 . 5}$ & 0.087 & 0.082 & 0.079 \\
\hline \multirow{5}{*}{$\mathbf{5 0}$} & $\mathbf{3 . 1}$ & 0.076 & 0.067 & 0.058 \\
\cline { 2 - 5 } & $\mathbf{4 . 8}$ & 0.079 & 0.072 & 0.064 \\
\cline { 2 - 5 } & $\mathbf{6}$ & 0.081 & 0.079 & 0.075 \\
\cline { 2 - 5 } & $\mathbf{8 . 5}$ & 0.072 & 0.065 & 0.063 \\
\hline
\end{tabular}

Tabla 4.6: Valor de RMSE para ajuste de la forma del espectro Doppler en el canal IB2OFF.

Para todas las distancias y frecuencias, se observa que el ajuste de la forma del espectro Doppler mediante la función de Laplace es la que mejor resultado presenta con un menor valor para el RMSE. En la Figura 4.17 se muestra gráficamente el valor medio del criterio de ajuste empleado para las tres funciones y las cuatro distancias de separación. 


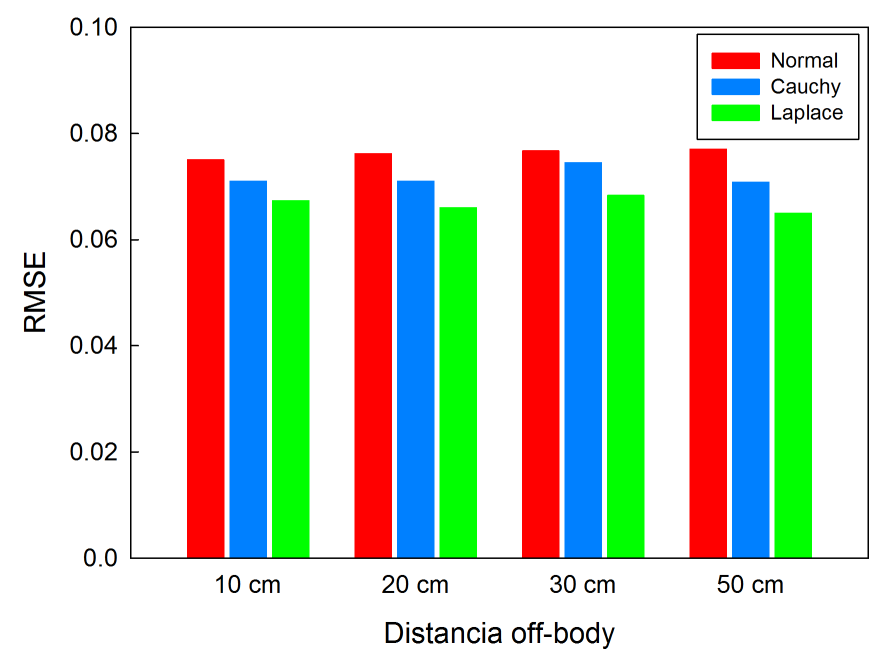

Figura 4.17: Valores de RMSE para el ajuste de la forma del espectro Doppler en el canal IB2OFF.

Independientemente de la distancia de separación entre Tx y Rx y la frecuencia de operación, como se observa en la Figura 4.14 el ajuste de la forma del espectro Doppler por medio de la función de Laplace es la que presenta el ajuste más óptimo con un valor medio de RMSE de 0.067 frente a 0.072 para Cauchy y 0.076 para la normal.

\subsubsection{Canal de propagación IB2IB}

Respecto al ajuste de la forma del espectro Doppler para el canal de propagación IB2IB, en la Tabla 4.7 se detalla el valor del criterio de ajuste RMSE sobre cada una de las funciones consideradas.

\begin{tabular}{|c|c|c|c|}
\hline $\begin{array}{c}\boldsymbol{f}_{\boldsymbol{c}} \\
(\mathbf{G H z})\end{array}$ & Normal & Cauchy & Laplace \\
\hline $\mathbf{3 . 1}$ & 0.081 & 0.134 & 0.124 \\
\hline $\mathbf{4 . 8}$ & 0.084 & 0.131 & 0.123 \\
\hline $\mathbf{6}$ & 0.088 & 0.107 & 0.101 \\
\hline $\mathbf{8 . 5}$ & - & - & - \\
\hline
\end{tabular}

Tabla 4.7: Valor de RMSE para ajuste de la forma del espectro Doppler en el canal IB2IB.

Al contrario de lo observado en los canales IB2OB e IB2OFF, en el caso del canal de propagación entre dos dispositivos ubicados en el interior del cuerpo, la función que mejor modela la forma del espectro Doppler es la normal. En la Figura 4.18 se muestra el ajuste a $3.1 \mathrm{GHz}$ de las diferentes funciones evaluadas sobre la forma del espectro Doppler en el canal IB2IB. 


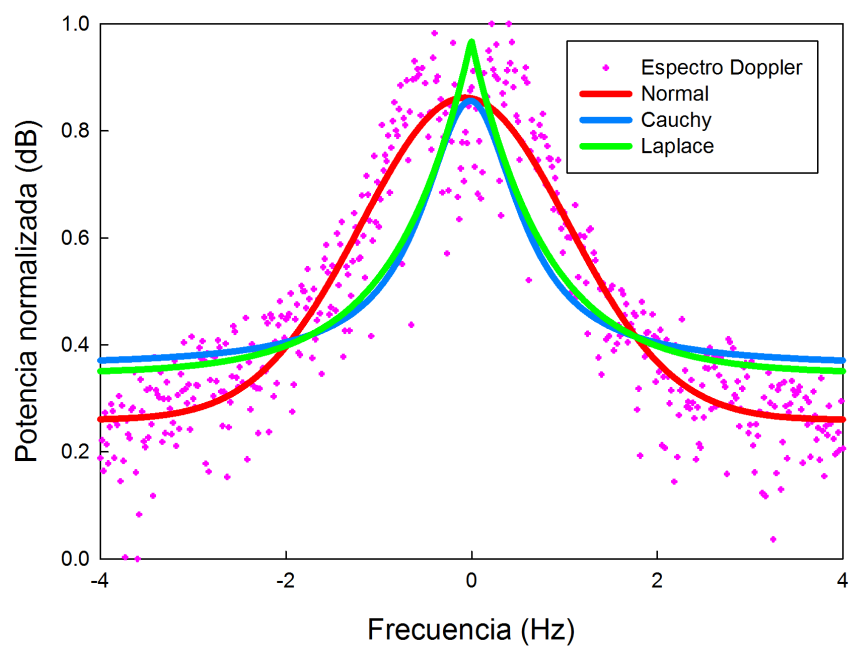

Figura 4.18: Ajuste de la forma del espectro Doppler para el canal IB2IB a $3.1 \mathrm{GHz}$.

A partir de $6 \mathrm{GHz}$ no es posible realizar el ajuste de la forma del espectro debido a que los valores de este provienen de muestras que se encuentran muy cerca o bajo el nivel de ruido del sistema.

\subsubsection{Estudio del ensanchamiento Doppler}

A partir del espectro Doppler para cada escenario de propagación, calculado por medio de la expresión (A.16) y normalizado en amplitud a $0 \mathrm{~dB}$, se han obtenido los parámetros de ensanchamiento Doppler spread y rms Doppler spread aplicando un umbral fijo de $-35 \mathrm{~dB}$ de manera que se considera ruido los valores bajo este y únicamente se tienen en cuenta las componentes frecuenciales cuyos niveles de potencia se encuentran por encima.

Con el fin de obtener valores teóricos de referencia para establecer un marco de comparación entre los valores de Doppler spread medidos frente a los primeros, en primer lugar se ha calculado el valor de ensanchamiento de referencia $\left(B_{\text {Dref }}\right)$ considerando la respiración un proceso sinusoidal perfecto, modelado por medio de la expresión (4.3), con la misma frecuencia que el proceso respiratorio real asociado y una excursión máxima entre Tx y Rx de $4 \mathrm{~cm}$. En la Figura 4.19 se muestra un ejemplo simplificado de un proceso teórico ideal de respiración (2 respiraciones/minuto). 


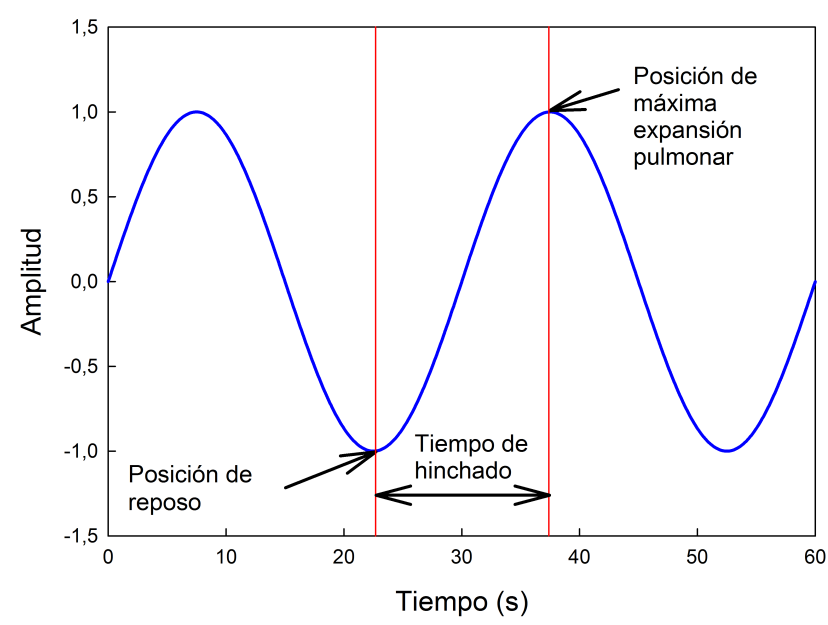

Figura 4.19: Modelo teórico de proceso respiratorio.

Una vez que se ha determinado la posición temporal de máxima y mínima expansión pulmonar, sabiendo que la excursión es de $4 \mathrm{~cm}$ se puede calcular la velocidad relativa $v$ de Tx hacia $\mathrm{Rx}$. A partir de esta velocidad y conociendo la frecuencia de operación $f_{c}$ y la permitividad relativa del phantom a esa frecuencia $\varepsilon_{r}\left(f_{c}\right)$ [Zhou et al., 2006], se puede calcular el máximo desplazamiento Doppler $f_{m}$ a partir de la expresión (A.15), y a partir de este el valor teórico de Doppler spread como $B_{\text {Dref }}=2 \cdot f_{m}$. A modo de referencia, y considerando una tasa de respiración de 12 respiraciones/minuto (proceso de $0.2 \mathrm{~Hz}$ ), en la Tabla 4.8 se muestran los valores teóricos para el Doppler spread a las frecuencias consideradas.

\begin{tabular}{|c|c|c|}
\hline $\begin{array}{c}\boldsymbol{f}_{\boldsymbol{c}} \\
(\mathbf{G H z})\end{array}$ & $\boldsymbol{\varepsilon}_{\boldsymbol{r}}$ & $\begin{array}{c}\boldsymbol{B}_{\text {Dref }} \\
(\mathbf{H z})\end{array}$ \\
\hline $\mathbf{3 . 1}$ & 59 & 2.54 \\
\hline $\mathbf{4 . 8}$ & 53 & 3.73 \\
\hline $\mathbf{6}$ & 49 & 4.48 \\
\hline $\mathbf{8 . 5}$ & 41 & 5.81 \\
\hline
\end{tabular}

Tabla 4.8: Valores de referencia del Doppler spread para 12 respiraciones/minuto.

Tal y como se observa, el valor de $B_{D}$ depende fuertemente de la frecuencia y en cualquier caso se encuentra por bajo de los $6 \mathrm{~Hz}$ bajo condiciones de respiración tranquila (12 respiraciones/minuto).

\subsubsection{Canal de propagación IB2OB}

En la Figura 4.20 se muestra los espectros Doppler correspondientes a las frecuencias 3.1 GHz, $4.8 \mathrm{GHz}, 6 \mathrm{GHz}$ y $8.5 \mathrm{GHz}$ para el canal de propagación IB2OB. 


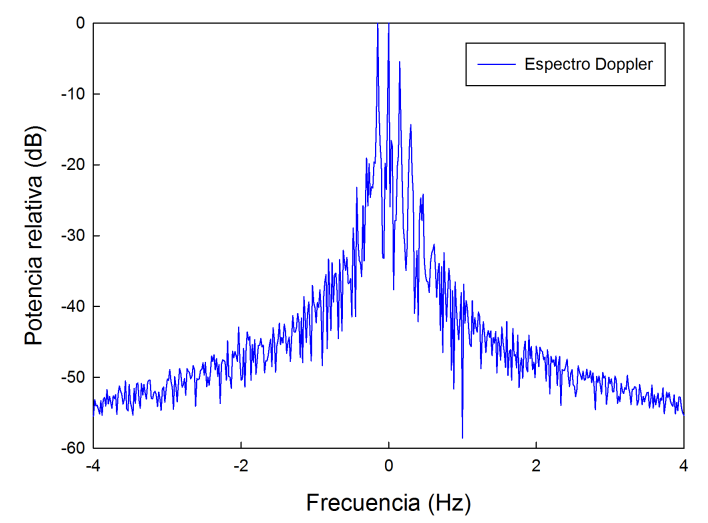

(a)

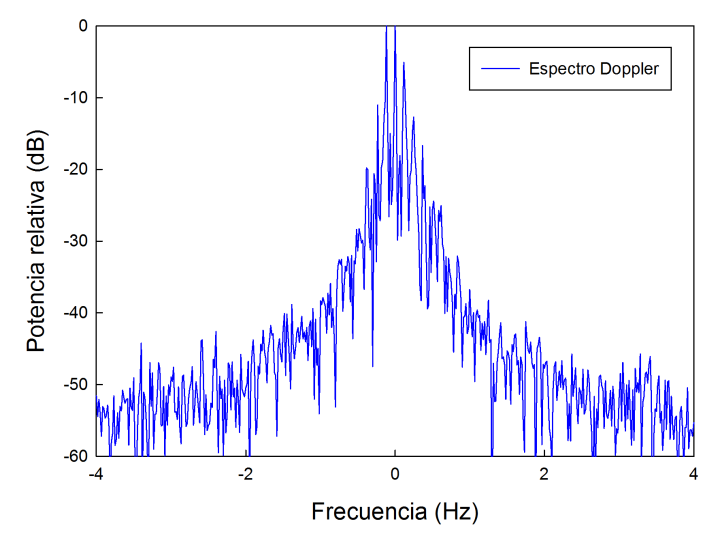

(c)

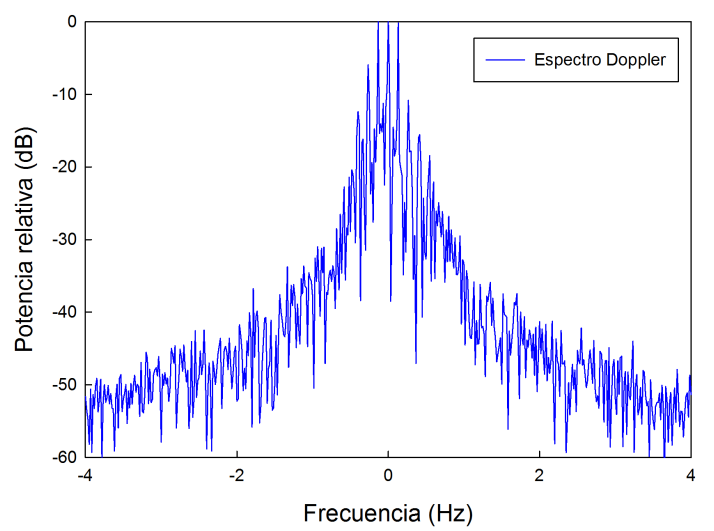

(b)

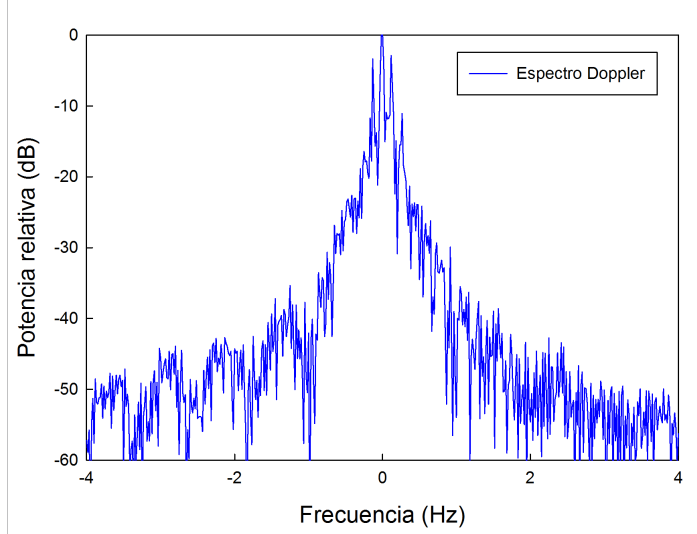

(d)

Figura 4.20: Espectros Doppler para el canal IB2OB. (a) $3.1 \mathrm{GHz}$, (b) $4.8 \mathrm{GHz}$, (c) $6 \mathrm{GHz}$ y (d) 8.5 $\mathrm{GHz}$.

Los parámetros de ensanchamiento en frecuencia del espectro Doppler, extraídos de las medidas, $B_{D}$ y $\rho_{r m s}$ se listan en la Tabla 4.9. Con el fin de establecer una referencia teórica para el valor del Doppler spread a cada una de las frecuencias, previamente se han calculado los valores teóricos de referencia. De esta forma se incluye el parámetro $\Delta B_{\text {Dref }}=\left(B_{D}-B_{\text {Dref }}\right)$ que representa la diferencia entre el valor teórico $\left(B_{D r e f}\right)$ y el real teniendo en cuenta el valor de la permitividad relativa del phantom a cada frecuencia y $\Delta B_{\text {Dref }_{0}}$ considerando un valor único de $\varepsilon_{r}=1$. Los valores negativos para estos parámetros indican un valor de ensanchamiento inferior al calculado teóricamente.

\begin{tabular}{|c|c|c|c|c|}
\hline $\begin{array}{c}\boldsymbol{f}_{\boldsymbol{c}} \\
(\mathbf{G H z})\end{array}$ & $\begin{array}{c}\boldsymbol{B}_{\boldsymbol{D}} \\
(\mathbf{H z})\end{array}$ & $\begin{array}{c}\Delta \boldsymbol{B}_{\text {Dref }} \\
\mathbf{( H z )}\end{array}$ & $\begin{array}{c}\Delta \boldsymbol{B}_{\text {Dref }} \\
(\mathbf{H z})\end{array}$ & $\begin{array}{c}\boldsymbol{\rho}_{\text {rms }} \\
\mathbf{( H z )}\end{array}$ \\
\hline $\mathbf{3 . 1}$ & 0.9 & -1.02 & 0.65 & 0.25 \\
\hline $\mathbf{4 . 8}$ & 1.25 & -1.2 & 0.91 & 0.36 \\
\hline $\mathbf{6}$ & 0.9 & -1.7 & 0.53 & 0.25 \\
\hline $\mathbf{8 . 5}$ & 1.08 & -2.74 & 0.49 & 0.32 \\
\hline
\end{tabular}

Tabla 4.9: Parámetros de ensanchamiento Doppler medidos para el canal IB2OB. 
Tal y como se observa comparando los valores medidos con los teóricos, el valor medido siempre es inferior al teórico considerando todo el canal de propagación entre Tx y $\mathrm{Rx}$ ocupado por el phantom, mientras que es superior al considerando propagación en el aire. Esto es previsible ya que únicamente un porcentaje del canal de propagación está ocupado por el phantom ( $9 \mathrm{~cm}$ con la antena Tx en posición de reposo), siendo el resto aire (4 $\mathrm{cm}$ por la pared del contenedor de $\varepsilon_{r} \approx 1$ ).

De manera general, conforme aumenta la frecuencia de operación aumentan las pérdidas en el canal y la amplitud de la señal recibida sufrirá mayor fluctuación debido a la cercanía al nivel de ruido tal y como se mostró en la Figura 4.10. Esto ocasiona en el dominio de la frecuencia un mayor ensanchamiento del espectro Doppler. En los valores medidos y listados en la Tabla 4.9, a partir de $4.8 \mathrm{GHz}$ no se observa de manera directa esta variación con la frecuencia. Esto puede ser debido a la respuesta en frecuencia de las antenas y al hecho de encontrarse en campo cercano.

\subsubsection{Canal de propagación IB2OFF}

En las Figura 4.21 y 4.22 se muestran los espectros Doppler correspondientes a las frecuencias $3.1 \mathrm{GHz}, 4.8 \mathrm{GHz}, 6 \mathrm{GHz}$ y $8.5 \mathrm{GHz}$ para el canal de propagación IB2OFF considerando las distancias de separación entre Tx y Rx $10 \mathrm{~cm}, 20 \mathrm{~cm}, 30 \mathrm{~cm}$ y $50 \mathrm{~cm}$.

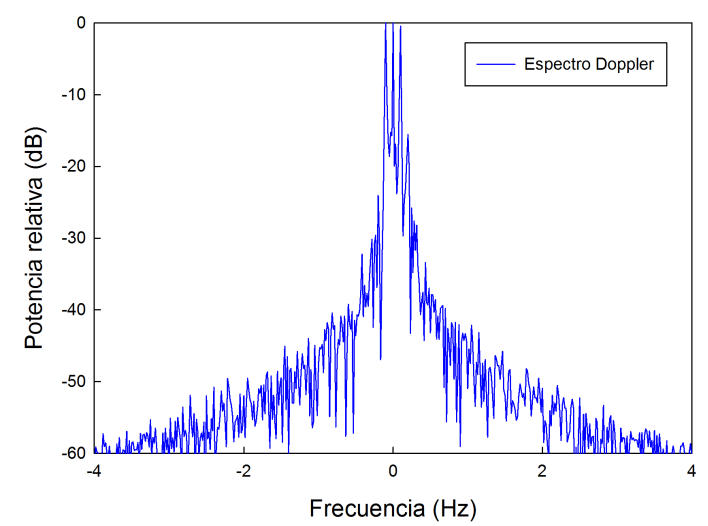

(a)

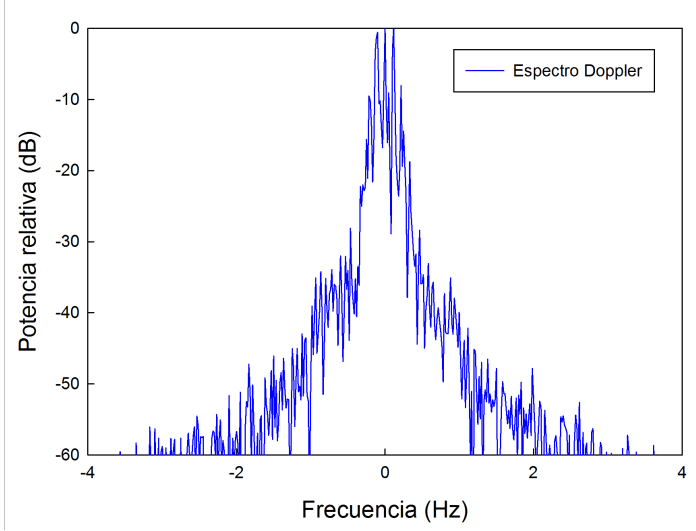

(b) 


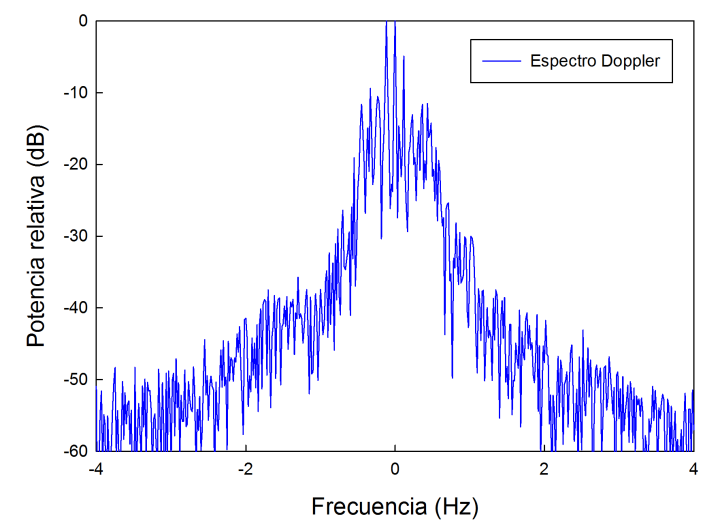

(c)

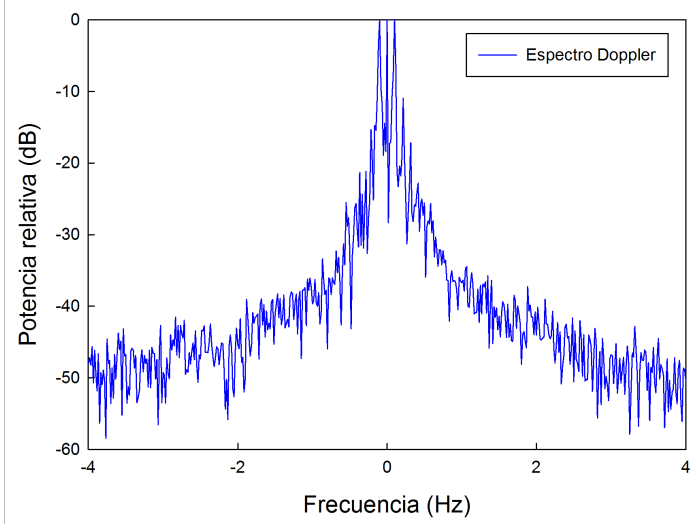

(d)

Figura 4.21: Espectros Doppler para el canal IB2OFF a $10 \mathrm{~cm}$. (a) $3.1 \mathrm{GHz}$, (b) $4.8 \mathrm{GHz}$, (c) $6 \mathrm{GHz}$ y (d) $8.5 \mathrm{GHz}$.

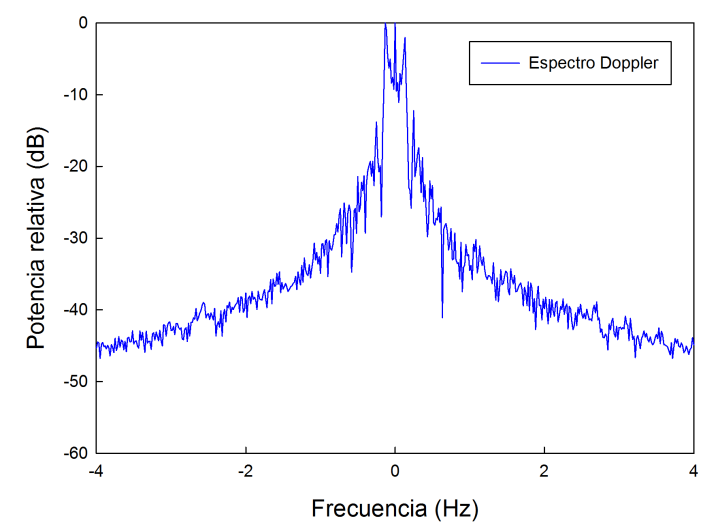

(a)

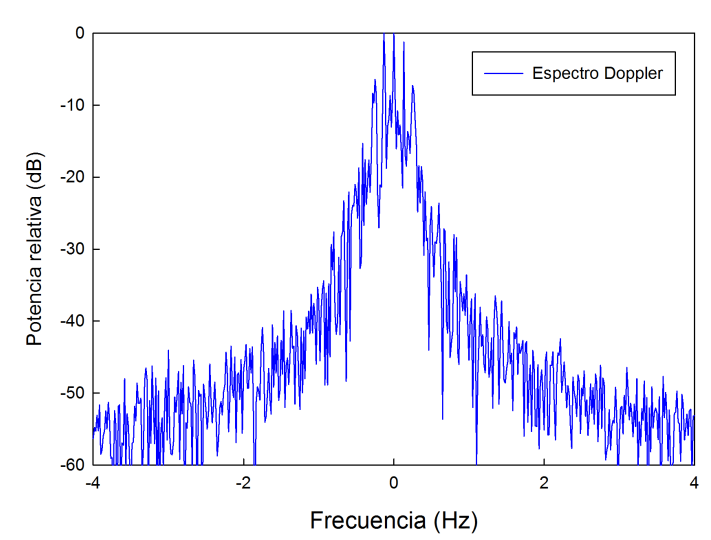

(c)

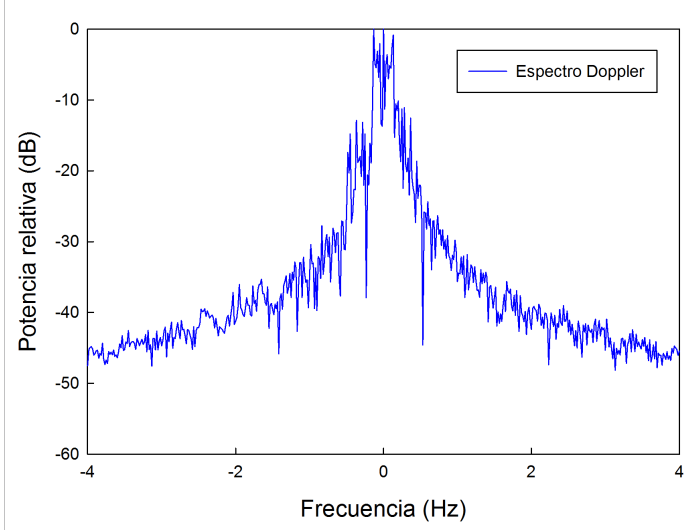

(b)

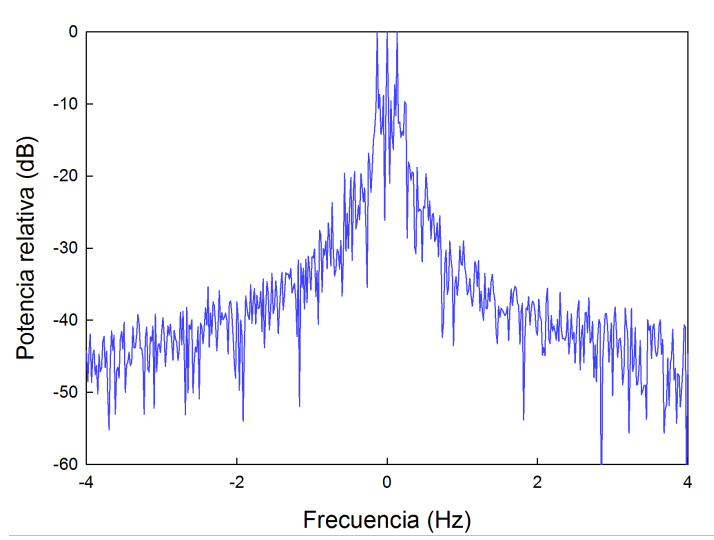

(d)

Figura 4.22: Espectros Doppler para el canal IB2OFF a $50 \mathrm{~cm}$. (a) $3.1 \mathrm{GHz}$, (b) $4.8 \mathrm{GHz}$, (c) $6 \mathrm{GHz}$ y (d) $8.5 \mathrm{GHz}$.

En la Tabla 4.10 se muestran los valores de ensanchamiento del espectro Doppler para el canal IB2OFF considerando las cuatro distancias de separación entre Tx y RX: $10 \mathrm{~cm}$, $20 \mathrm{~cm}, 30 \mathrm{~cm}$ y $50 \mathrm{~cm}$. Al igual que en el caso del canal IB2OB se incluyen los parámetros 
$B_{\text {Dref }}$ y $B_{\text {Dref } \varepsilon_{0}}$ para establecer una comparación entre el valor medido respecto del calculado teóricamente considerando la permitividad relativa del phantom a la frecuencia de operación y la permitividad relativa del aire.

\begin{tabular}{|c|c|c|c|c|c|}
\hline $\begin{array}{c}\text { Distancia } \\
\text { Tx-Rx } \\
(\mathbf{c m})\end{array}$ & $\begin{array}{c}\boldsymbol{f}_{\boldsymbol{c}} \\
(\mathbf{G H z})\end{array}$ & $\begin{array}{c}\boldsymbol{B}_{\boldsymbol{D}} \\
(\mathbf{H z})\end{array}$ & $\begin{array}{c}\Delta \boldsymbol{B}_{\text {Dref }} \\
\mathbf{( H z )}\end{array}$ & $\begin{array}{c}\Delta \boldsymbol{B}_{\text {Dref }} \\
(\mathbf{H z})\end{array}$ & $\begin{array}{c}\boldsymbol{\rho}_{\text {rms }} \\
(\mathbf{H z})\end{array}$ \\
\hline \multirow{4}{*}{$\mathbf{1 0}$} & $\mathbf{3 . 1}$ & 0.42 & -0.85 & 0.25 & 0.12 \\
\cline { 2 - 6 } & $\mathbf{4 . 8}$ & 0.67 & -1.5 & 0.38 & 0.21 \\
\cline { 2 - 6 } & $\mathbf{6}$ & 1.15 & -1.45 & 0.78 & 0.35 \\
\cline { 2 - 6 } & $\mathbf{8 . 5}$ & 0.78 & -2.6 & 0.25 & 0.23 \\
\hline \multirow{4}{*}{$\mathbf{2 0}$} & $\mathbf{3 . 1}$ & 0.85 & -0.42 & 0.68 & 0.23 \\
\cline { 2 - 6 } & $\mathbf{4 . 8}$ & 1.23 & -0.94 & 0.94 & 0.35 \\
\cline { 2 - 6 } & $\mathbf{6}$ & 1.28 & -1.32 & 0.91 & 0.39 \\
\cline { 2 - 6 } & $\mathbf{8 . 5}$ & 0.85 & -2.53 & 0.32 & 0.23 \\
\hline \multirow{4}{*}{$\mathbf{3 0}$} & $\mathbf{3 . 1}$ & 1.0 & -0.27 & 0.83 & 0.31 \\
\cline { 2 - 6 } & $\mathbf{4 . 8}$ & 1.23 & -0.94 & 0.94 & 0.33 \\
\cline { 2 - 6 } & $\mathbf{6}$ & 1.63 & -0.97 & 1.26 & 0.44 \\
\cline { 2 - 6 } & $\mathbf{8 . 5}$ & 1.02 & -2.36 & 0.49 & 0.30 \\
\hline \multirow{4}{*}{$\mathbf{5 0}$} & $\mathbf{3 . 1}$ & 1.0 & -0.48 & 0.81 & 0.32 \\
\cline { 2 - 6 } & $\mathbf{4 . 8}$ & 1.08 & -1.09 & 0.79 & 0.33 \\
\cline { 2 - 6 } & $\mathbf{6}$ & 1.27 & -1.33 & 0.90 & 0.34 \\
\cline { 2 - 6 } & $\mathbf{8 . 5}$ & 1.3 & -2.08 & 0.77 & 0.36 \\
\hline
\end{tabular}

Tabla 4.10: Parámetros de ensanchamiento Doppler medidos para el canal IB2OFF.

Respecto a la variación del ensanchamiento del espectro Doppler con la frecuencia de operación, se observa que, a excepción de los valores para la frecuencia de $8.5 \mathrm{GHz}$, sí se observa el aumento en los valores de los parámetros de ensanchamiento $B_{D}$ y $\rho_{r m s}$. Los valores medidos, al igual que en el caso del canal IB2OB, son inferiores a los teóricos considerando todo el medio ocupado por el phantom y superiores a los correspondientes a propagación en el aire. De las distancias consideradas, únicamente en $50 \mathrm{~cm}$ para frecuencias inferiores a $6 \mathrm{GHz}$ se puede considerar que las antenas se encuentran en campo lejano $\left(R \gg 2 D^{2} / \lambda\right)$. Debido a que no todo el canal está ocupado por el phantom, la distancia $R$ para la cual las antenas se pueden considerar así para $4.8 \mathrm{GHz}$ será superior a $8 \mathrm{~cm}$ (con $\left.\varepsilon_{r}=1\right)$ e inferior a $58 \mathrm{~cm}\left(\varepsilon_{r} \approx 53\right)$.

En la Figura 4.23 se detalla la variación del valor del Doppler spread con la distancia a cada frecuencia considerada. 


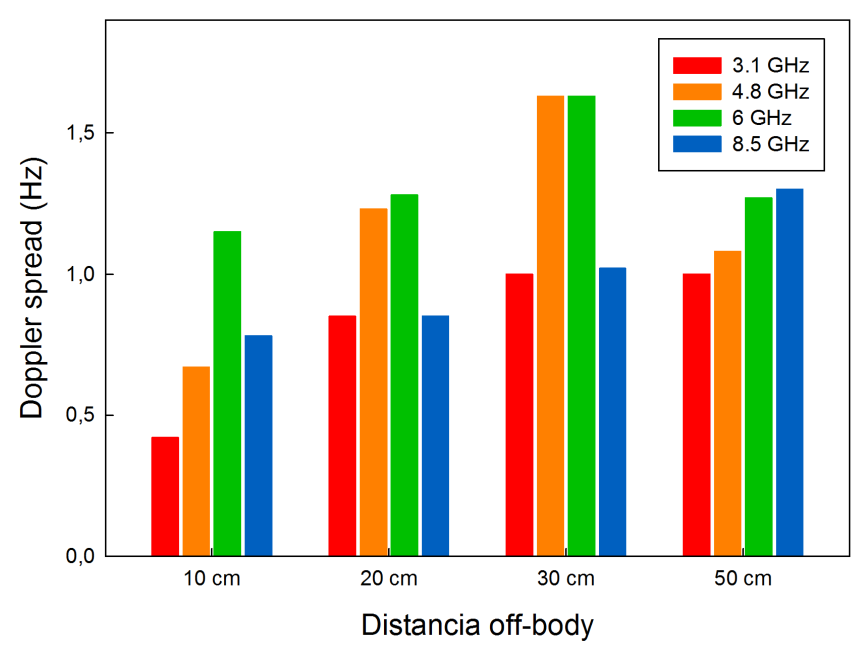

Figura 4.23: Variación del Doppler spread con la distancia en el canal IB2OFF.

Como se observa en la Figura 4.23, únicamente a $50 \mathrm{~cm}$ (condiciones de propagación aproximadamente en campo lejano) el valor del Doppler spread aumenta de manera homogénea con la frecuencia de operación. Para distancias inferiores, únicamente se observa este aumento en el rango de frecuencias de 3.1-6 GHz aunque con una mayor desviación típica $\sigma$ en el incremento por distancia $(0.24-0.37 \mathrm{~Hz}$ frente a $0.14 \mathrm{~Hz}$ para $50 \mathrm{~cm})$.

\subsubsection{Canal de propagación IB2IB}

En la Figura 4.24 se muestra los espectros Doppler correspondientes a las frecuencias 3.1 GHz, $4.8 \mathrm{GHz}, 6 \mathrm{GHz}$ y $8.5 \mathrm{GHz}$ para el canal de propagación IB2IB.

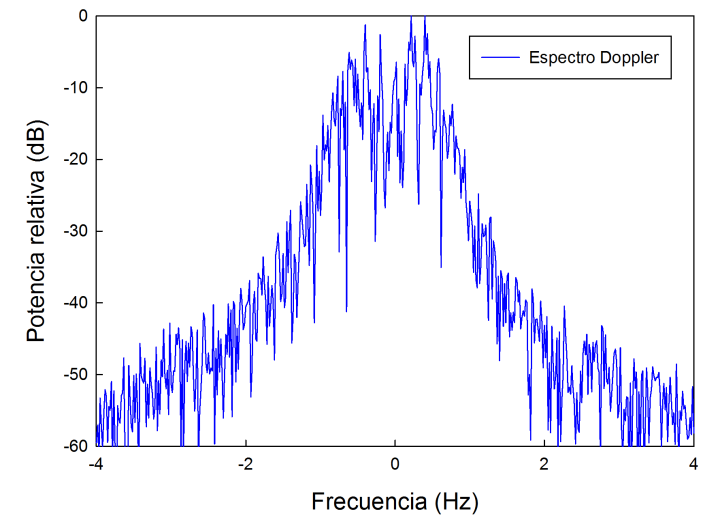

(a)

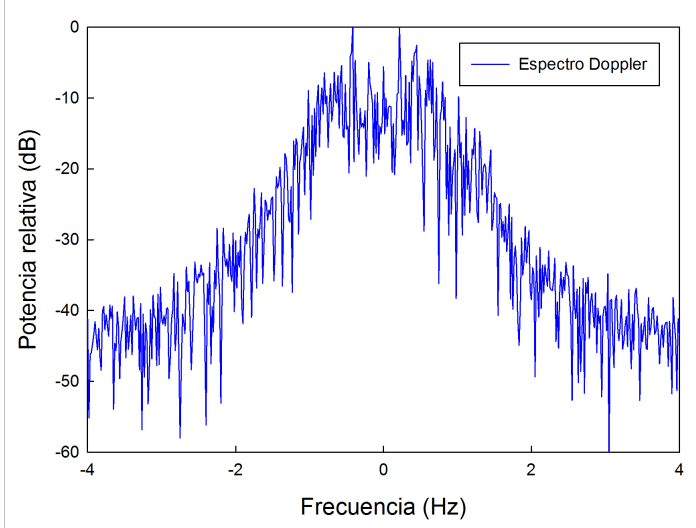

(b) 


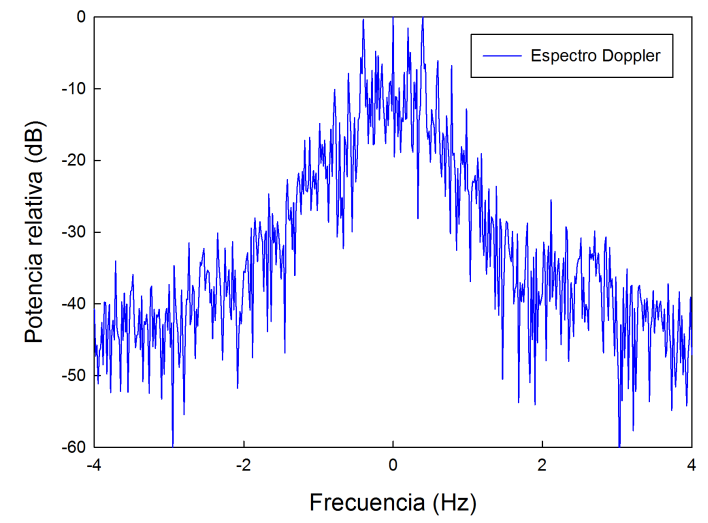

(c)

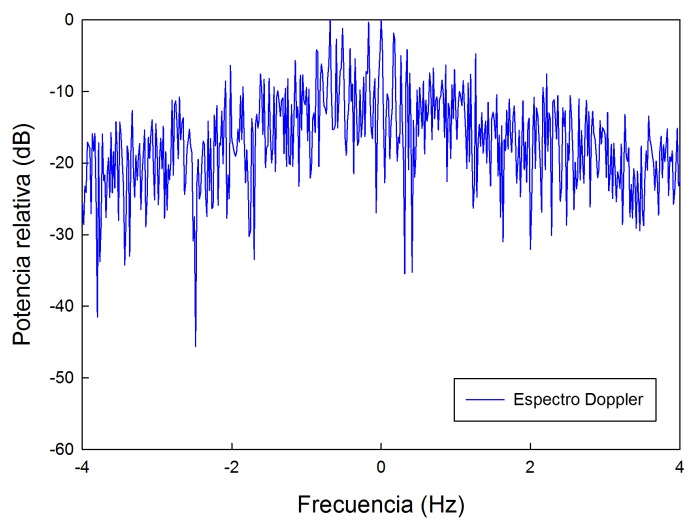

(d)

Figura 4.24: Espectros Doppler para el canal IB2IB. (a) $3.1 \mathrm{GHz}$, (b) $4.8 \mathrm{GHz}$, (c) $6 \mathrm{GHz}$ y (d) 8.5 $\mathrm{GHz}$.

En la Tabla 4.11 se muestran los valores de ensanchamiento del espectro Doppler para el canal IB2IB con las dos antenas sumergidas en el phantom. Además, se incluye el parámetro $\Delta B_{\text {Dref }}$ para establecer el valor de referencia teórico teniendo en cuenta el valor de la permitividad relativa del phantom a cada frecuencia considerada.

\begin{tabular}{|c|c|c|c|}
\hline $\begin{array}{c}\boldsymbol{f}_{\boldsymbol{c}} \\
(\mathbf{G H z})\end{array}$ & $\begin{array}{c}\boldsymbol{B}_{\boldsymbol{D}} \\
(\mathbf{H z})\end{array}$ & $\begin{array}{c}\Delta \boldsymbol{B}_{\text {Dref }} \\
(\mathbf{H z})\end{array}$ & $\begin{array}{c}\boldsymbol{\rho}_{\text {rms }} \\
\mathbf{( H z )}\end{array}$ \\
\hline $\mathbf{3 . 1}$ & 2.30 & -0.24 & 0.65 \\
\hline $\mathbf{4 . 8}$ & 3.45 & -0.27 & 0.98 \\
\hline $\mathbf{6}$ & 3.05 & -1.43 & 0.82 \\
\hline $\mathbf{8 . 5}$ & - & - & - \\
\hline
\end{tabular}

Tabla 4.11: Parámetros de ensanchamiento Doppler medidos para el canal IB2IB.

$\mathrm{Al}$ encontrarse ambas antenas en el interior del phantom, el efecto de campo cercano es más significativo que en el resto de escenarios de propagación. En este caso únicamente la frecuencia de $3.1 \mathrm{GHz}$ se puede considerar hábil, debido a que a frecuencias superiores, tal y como se observa en la Figura 4.14 b, c y d y la Figura 4.24 b, c y d, la potencia de la señal recibida se encuentra muy cercana o bajo el nivel de ruido del sistema. De la Tabla 4.11 se deduce que el valor de Doppler spread obtenido y el teórico son similares, ya que para el cálculo de este último se ha tenido en cuenta la permitividad relativa del phantom a esa frecuencia. Esto confirma además, que el valor de $\varepsilon_{r}=59$ para la frecuencia de $3.1 \mathrm{GHz}$ reportado en [Zhou et al., 2006] para el phantom empleado es muy aproximado al existente en este caso. 


\subsection{Caracterización en el tiempo del canal in-body dinámico}

El estudio del efecto del movimiento relativo entre una antena transmisora y otra receptora considerando al menos una de ellas colocada en el interior del cuerpo, debido a respiración se puede realizar desde la perspectiva de la variación temporal del canal radio por medio de la función de autocorrelación y el tiempo de coherencia $T_{c}$. Debido a que el proceso respiratorio es de carácter periódico, la función de autocorrelación del canal $R_{H}(t)$ también lo será. De manera ideal, considerando el modelo teórico de la expresión (4.3), la función de autocorrelación $R_{x x}(\lambda)$ se puede calcular como la siguiente esperanza matemática,

$$
R_{x x}(\lambda)=E\left\{f^{*}(t) \cdot f(t+\lambda)\right\}
$$

Suponiendo $f(t)$ determinista, la autocorrelación puede calcularse integrando en un periodo de la función de la siguiente forma,

$$
R_{x x}(\lambda)=\frac{1}{T_{c}} \int_{0}^{T_{c}} f(t) \cdot f(t+\lambda) d t
$$

Por lo tanto, considerando el modelo teórico para la variación en la amplitud de la señal recibida presentado en la sección 4.4.1, $f(t)=A+\alpha \cdot \operatorname{sen}(2 \pi \beta t+\varphi)$, se obtiene que la función de autocorrelación de la señal recibida es de la siguiente forma,

$$
R_{x x}(\lambda)=C t e \cdot \cos (2 \pi \beta \lambda)
$$

donde Cte es una constante que depende de $A$ y de $\alpha$. En la realidad, debido a que se considera una señal recibida limitada en el tiempo, cuya amplitud además está afectada por desvanecimiento causado por efectos de la respuesta del canal a la frecuencia considerada, efectos de campo cercano, etc. la función de autocorrelación real $R_{H}(t)$ calculada por medio de la expresión (A.18) a partir de las muestras obtenidas del parámetro de dispersión $S_{21}$ medido con el analizador de redes vectorial, tenderá a 0 en el extremo del intervalo $(60 \mathrm{~s})$. En la Figura 4.25 se muestra un ejemplo de $R_{H}(t)$, donde además se muestra el ajuste de la envolvente de la misma por medio de la función exponencial siguiente,

$$
f(\lambda)=k \cdot e^{-\frac{\lambda}{\Gamma}}
$$


donde $\Gamma$ es la tasa de decaimiento de la función de autocorrelación.

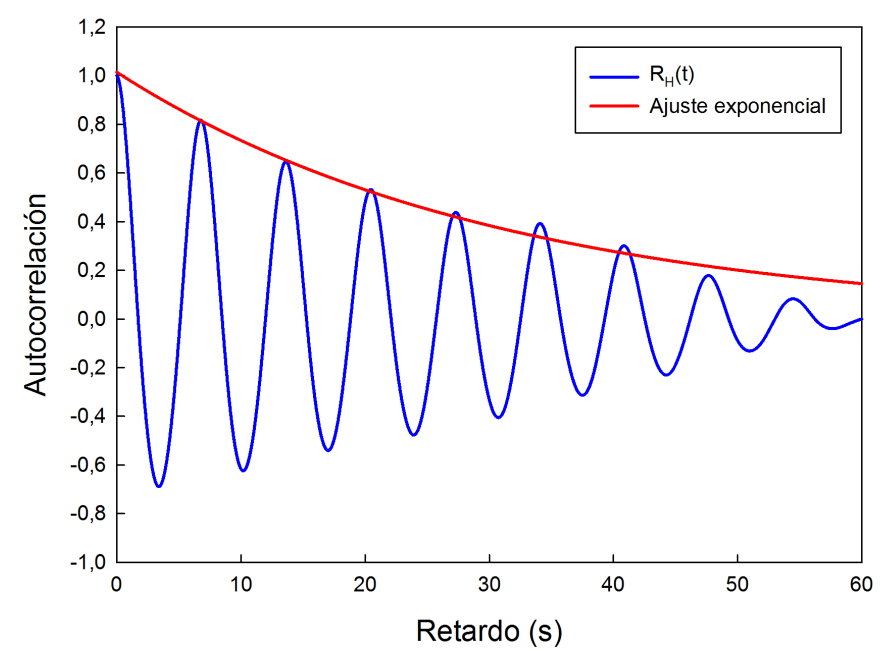

Figura 4.25: Función de autocorrelación del proceso respiratorio real y ajuste exponencial.

A partir de la función de autocorrelación, se puede obtener el tiempo de coherencia del canal $T_{c}$ como el retardo en el cual la función tiene un valor (por primera vez) inferior a un cierto umbral (entre 0.5 y 0.9 ). Este parámetro se relaciona de manera inversa con el ensanchamiento Doppler $\left(T_{c}=1 / B_{D}\right)$ observado en la señal recibida, de manera que conforme aumenta el ensanchamiento del espectro Doppler, menor es el tiempo de coherencia del canal, por lo que además, se reducirá la máxima tasa posible de transferencia en el canal libre de errores (sin emplear técnicas de ecualización del canal).

Con el fin de realizar la caracterización de la variación temporal en el canal derivada del movimiento relativo entre antenas debido a la respiración, se han calculado las funciones de autocorrelación $R_{x x}(\lambda)$ correspondientes a cada una de las medidas previamente filtradas, empleando el filtro de media móvil de la expresión (4.1) con un tamaño de ventana $L=20$, de las respuestas al impulso variables en el tiempo $H\left(f_{c}, t\right)$ obtenidas mediante el analizador de redes vectorial, configurado este en modo de onda continua con los parámetros detallados en la Tabla 4.1. Tal y como se describió en la sección 4.4, para el caso del canal IB2OB la antena transmisora se ha colocado en el interior del contenedor, inmersa en el phantom líquido y acoplada a la esfera hinchable de látex mediante adhesivo, mientras que la antena receptora se ha colocado sobre la pared exterior del contenedor, orientada hacia Tx, a una distancia inicial de $13 \mathrm{~cm}$, tal y como se detalla en la Figura 4.6. Para las medidas del canal IB2OFF, al igual que en el caso anterior, la antena Tx se ha colocado inmersa en el phantom pero en este caso la antena Rx se ha colocado sobre un soporte a cuatro distancias de separación diferentes respecto al contenedor: $10 \mathrm{~cm}, 20 \mathrm{~cm}, 30 \mathrm{~cm}$ y $50 \mathrm{~cm}$, tal y como se 
detalla en la Figura 4.7. Por último, para las medidas del canal IB2IB, ambas antenas se han ubicado en el interior del contenedor, inmersas en el phantom líquido guardando una separación inicial entre ellas de $7 \mathrm{~cm}$. El esquema detallado de los elementos para estas medidas se muestra en la Figura 4.8. Para los tres escenarios anteriores la extensión del recorrido entre $\mathrm{Tx}$ y $\mathrm{Rx}$ se ha restringido a $4 \mathrm{~cm}$ para emular la expansión torácica humana habitual. Además, la respuesta del canal se ha medido en un intervalo de $60 \mathrm{~s}$ durante el cual, se ha insuflado/liberado de manera cíclica aire en el interior de la esfera de látex empleando el tubo de conexión simulando el hinchado/deshinchado de un pulmón durante las fases de inspiración y espiración.

\subsubsection{Caracterización de la autocorrelación en el canal IB2OB}

A partir de la función de autocorrelación $R_{H}(t)$ para las medidas realizadas en el caso del canal de propagación IB2OB se ha calculado el tiempo de coherencia $T_{c}$ teniendo en cuenta diferentes valores de umbral entre 0.5 y 0.9 . Además, se ha obtenido la tasa de decaimiento $\Gamma$ de la función de autocorrelación mediante del ajuste de la envolvente por medio de la función exponencial (4.13). Los valores se listan en la Tabla 4.12.

\begin{tabular}{|c|c|c|c|c|c|c|}
\hline $\begin{array}{c}\boldsymbol{f}_{\boldsymbol{c}} \\
(\mathbf{G H z})\end{array}$ & $\begin{array}{c}\boldsymbol{T}_{c 05} \\
(\mathbf{s})\end{array}$ & $\begin{array}{c}\boldsymbol{T}_{c 06} \\
(\mathbf{s})\end{array}$ & $\begin{array}{c}\boldsymbol{T}_{c 07} \\
(\mathbf{s})\end{array}$ & $\begin{array}{c}\boldsymbol{T}_{c 08} \\
\mathbf{( s )}\end{array}$ & $\begin{array}{c}\boldsymbol{T}_{c 09} \\
(\mathbf{s})\end{array}$ & $\Gamma$ \\
\hline $\mathbf{3 . 1}$ & 1.07 & 0.93 & 0.78 & 0.63 & 0.42 & 30.9 \\
\hline $\mathbf{4 . 8}$ & 0.94 & 0.80 & 0.65 & 0.51 & 0.34 & 24.5 \\
\hline $\mathbf{6}$ & 1.16 & 1.01 & 0.83 & 0.65 & 0.41 & 24.5 \\
\hline $\mathbf{8 . 5}$ & 1.63 & 1.36 & 1.08 & 0.82 & 0.51 & 8.7 \\
\hline
\end{tabular}

Tabla 4.12: Tiempos de coherencia según umbral y tasa de decaimiento de la autocorrelación para el canal IB2OB.

El desplazamiento temporal a partir del cual dos señales dejan de presentar un cierto nivel de correlación en el canal IB2OB es cada vez menor conforme aumenta la frecuencia de operación, debido al desvanecimiento en el canal. Los valores de desplazamiento Doppler máximo $f_{m}$ calculado como $B_{D} / 2$ a partir de los valores listados en la Tabla 4.9, para cada una de las frecuencias, son $0.45 \mathrm{~Hz}, 0.63 \mathrm{~Hz}, 0.45 \mathrm{~Hz}$ y $0.54 \mathrm{~Hz}$ respectivamente para $3.1 \mathrm{GHz}, 4.8 \mathrm{GHz}, 6 \mathrm{GHz}$ y $8.5 \mathrm{GHz}$. A partir de estos valores, y considerando la expresión (A.20) con $k=0.423$ se obtienen los valores de tiempo de coherencia: $0.94 \mathrm{~s}, 0.68 \mathrm{~s}, 0.94 \mathrm{~s}$ y 0.78 s. Considerando los valores de la Tabla 4.12 se observa que esos valores corresponden aproximadamente a los tiempos de coherencia para un nivel de correlación de 0.7. Empleando este nivel, el error medio entre los valores medidos para $T_{c}$ a partir del espectro Doppler y los obtenidos a partir de la función de autocorrelación presentan una variación media de $0.15 \mathrm{~s}$. 


\subsubsection{Caracterización de la autocorrelación en el canal IB2OFF}

Considerando un nivel de 0.7 para la obtención del tiempo de coherencia a partir de la función de autocorrelación, en la Tabla 4.13 se detallan los valores tanto para $T_{c}$ como para $\Gamma$ correspondientes a las cuatro distancias de separación entre Tx y Rx para el caso del canal de propagación IB2OFF a las frecuencias consideradas.

\begin{tabular}{|c|c|c|c|c|}
\hline & \multicolumn{4}{|c|}{$\boldsymbol{T}_{\boldsymbol{c} 07}(\boldsymbol{s}) / \Gamma$} \\
\hline $\begin{array}{c}\boldsymbol{f}_{\boldsymbol{c}} \\
(\mathbf{G H z})\end{array}$ & $\mathbf{1 0} \mathbf{~ c m}$ & $\mathbf{2 0} \mathbf{c m}$ & $\mathbf{3 0} \mathbf{~ c m}$ & $\mathbf{5 0} \mathbf{~ c m}$ \\
\hline $\mathbf{3 . 1}$ & $1.19 / 33.59$ & $1.24 / 19.04$ & $1.13 / 12.97$ & $1.0 / 18.26$ \\
\hline $\mathbf{4 . 8}$ & $1.01 / 18.48$ & $0.91 / 21.15$ & $0.75 / 32.08$ & $0.96 / 17.49$ \\
\hline $\mathbf{6}$ & $0.53 / 17.72$ & $0.55 / 20.41$ & $0.69 / 27.62$ & $0.67 / 14.66$ \\
\hline $\mathbf{8 . 5}$ & $1.04 / 31.58$ & $0.82 / 30.07$ & $1.24 / 8.73$ & $0.76 / 27.1$ \\
\hline
\end{tabular}

Tabla 4.13: Tiempos de coherencia según umbral y tasa de decaimiento de la autocorrelación para el canal IB2OFF.

Tal y como se observa en la Tabla 4.13, considerando un nivel correlación de 0.7 se obtiene una variación media de $0.21 \mathrm{~s}$ en la estima de $T_{c}$ respecto al valor obtenido a partir del espectro Doppler empleando la expresión (A.20). Al igual que se ha comentado en el punto 4.5.2.2 respecto al valor de $B_{D}$, la variación del tiempo de coherencia con la frecuencia y la distancia presenta una alta variabilidad para distancias inferiores a $50 \mathrm{~cm}$ tal y como se muestra en la Figura 4.26, la cual está relacionada con la Figura 4.23 debido a la relación inversa ente $T_{c} \mathrm{y} B_{D}$.

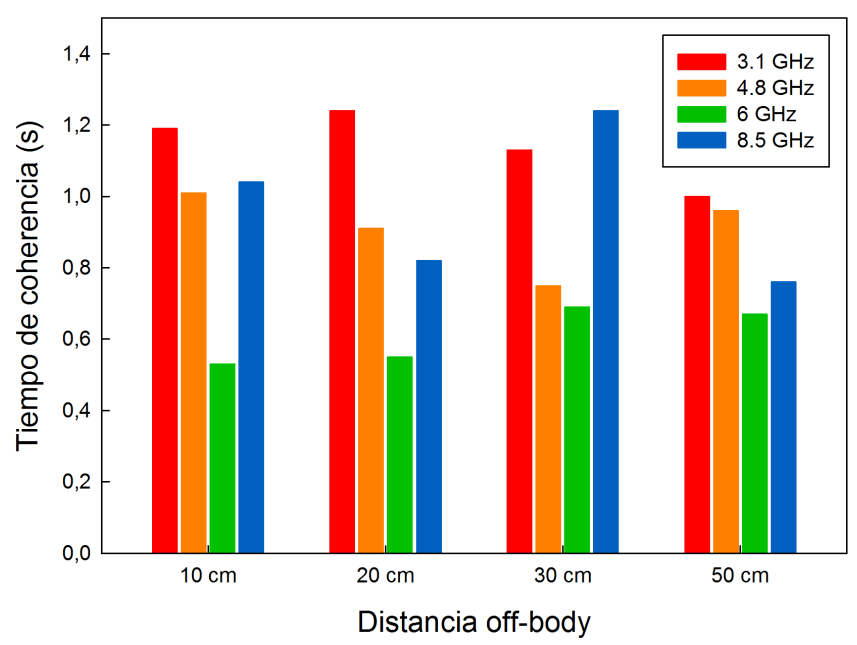

Figura 4.26: Variación del tiempo de coherencia con la distancia en el canal IB2OFF. 
En general, para todas las distancias se observa una reducción en el tiempo de coherencia del canal conforme aumenta la frecuencia de operación, a excepción de $8.5 \mathrm{GHz}$. Además, tal y como se observa en la Figura 4.26, únicamente a $50 \mathrm{~cm}$ la reducción es más homogénea, probablemente debido a las condiciones de propagación en campo lejano por parte de las antenas. Por último, la reducción en el tiempo de coherencia conforme aumenta la distancia de separación no parece seguir una tendencia clara a partir de $4.8 \mathrm{GHz}$.

\subsubsection{Caracterización de la autocorrelación en el canal IB2IB}

Los valores para el tiempo de coherencia $T_{c}$ para un nivel de correlación de 0.7 y $\Gamma$ para el caso del canal de propagación IB2IB se encuentran listados en la Tabla 4.14.

\begin{tabular}{|c|c|c|}
\hline $\begin{array}{c}\boldsymbol{f}_{\boldsymbol{c}} \\
(\mathbf{G H z})\end{array}$ & $\begin{array}{c}\boldsymbol{T}_{\boldsymbol{c 0 7}} \\
(\mathbf{s})\end{array}$ & $\Gamma$ \\
\hline $\mathbf{3 . 1}$ & 0.29 & 5.34 \\
\hline $\mathbf{4 . 8}$ & 0.22 & 1.85 \\
\hline $\mathbf{6}$ & 0.28 & 9.09 \\
\hline $\mathbf{8 . 5}$ & ND & ND \\
\hline
\end{tabular}

Tabla 4.14: Tiempos de coherencia según umbral y tasa de decaimiento de la autocorrelación para el canal IB2IB.

Como se observa en la Tabla 4.14, considerando un nivel correlación de 0.7 se obtiene una variación medio de $0.03 \mathrm{~s}$ en la estima de $T_{c}$ respecto al valor obtenido a partir del espectro Doppler empleando la expresión (A.20). A $8.5 \mathrm{GHz}$ no es posible determinar el tiempo de coherencia del canal puesto que las muestras obtenidas se encuentran bajo el umbral de ruido del sistema.

\subsection{Conclusiones}

En este capítulo se han presentado los resultados de la caracterización de los efectos sobre el canal de propagación in-body derivados del movimiento relativo entre dos dispositivos de una WBAN por causa de la respiración. Se han considerado diferentes enlaces entre dos dispositivos, uno de ellos ubicado en el interior del cuerpo y otro dispositivo colocado sobre la superficie (canal IB2OB), en el exterior a una cierta distancia de separación respecto al cuerpo (canal IB2OFF) y, por último, también en el interior del mismo (canal IB2IB). Para ello se ha empleado un phantom líquido diseñado para emular las características dieléctricas de los tejidos humanos con alto contenido en agua en la banda de UWB de 3.1-10.6 GHz. El estudio se ha llevado a cabo atendiendo a los efectos en el dominio de la frecuencia y el tiempo derivados del movimiento entre dispositivos. En este sentido, los 
efectos en el dominio de la frecuencia se han caracterizado por medio del estudio del espectro Doppler de la señal recibida a partir del modelado de la forma del mismo y los parámetros Doppler spread y ensanchamiento rms y, por otro lado, el efecto en el tiempo se ha caracterizado por medio de la función de autocorrelación y el tiempo de coherencia del canal radio. Adicionalmente, se ha realizado el modelado del proceso respiratorio en base a una función periódica sinusoidal cuyos parámetros se han calculado para cada escenario de propagación.

En el canal de propagación IB2OB, cuando ambas antenas se encuentran en movimiento relativo debido a la respiración, aunque teóricamente se debería observar un aumento en el Doppler spread $B_{D}$ con la frecuencia, a partir de $4.8 \mathrm{GHz}$ esto no se observa de manera clara. La causa de este comportamiento puede deberse a la respuesta en frecuencia y la interacción en campo cercano de las antenas empleadas. Respecto al modelado de la forma del espectro Doppler, se ha observado que la función de Laplace es la que proporciona un ajuste más óptimo comparado con la normal o la función de Cauchy. Además, del estudio de la función de autocorrelación, se ha obtenido valores entre $0.65 \mathrm{~s}$ y $1.08 \mathrm{~s}$ para el tiempo de coherencia $T_{c}$ considerando un nivel de correlación del 0.7 , lo cual supone una variación de $0.15 \mathrm{~s}$ respecto al valor de $T_{c}$ si se obtiene este de manera teórica partir de la relación inversa con el Doppler spread .

El canal IB2OFF entre una antena colocada en el interior del cuerpo y otra en el exterior se ha estudiado considerando cuatro distancias de separación: $10 \mathrm{~cm}, 20 \mathrm{~cm}, 30 \mathrm{~cm}$ y $50 \mathrm{~cm}$. Al igual que se ha observado en el canal IB2OB, la función que mejor se ajusta a la forma del espectro Doppler a todas las distancias y frecuencias consideradas es la de Laplace. Respecto a la variación de $B_{D}$ se puede establecer que para una distancia de separación de $50 \mathrm{~cm}$, el ensanchamiento Doppler es incremental de manera clara con la frecuencia. Esto puede deberse al hecho de que a esa distancia las antenas se encuentran en campo lejano o muy cercano a este por lo que las características de radiación de las antenas son más estables. Por el contrario, para distancias inferiores este aumento únicamente se da para frecuencias inferiores a $8.5 \mathrm{GHz}$ y presenta una mayor desviación típica ( $\sigma \in[0.24 \mathrm{~Hz}$, $0.37 \mathrm{~Hz}])$ comparado con el caso a $50 \mathrm{~cm}(\sigma=0.14 \mathrm{~Hz})$. Esto puede ser consecuencia de encontrarse las antenas en campo cercano. En cuanto a la variación temporal del canal se observa el mismo comportamiento respecto al valor de $B_{D}$ descrito para $50 \mathrm{~cm} \mathrm{y} \mathrm{las}$ distancias inferiores. Considerando un nivel de correlación de 0.7 , el valor de $T_{c}$ varía entre $1.24 \mathrm{~s}$ y $0.53 \mathrm{~s}$. Además, a excepción de a $8.5 \mathrm{GHz}$ se observa una reducción en el tiempo de 
coherencia del canal conforme aumenta la frecuencia. Por el contrario, la variación conforme aumenta la distancia off-body no es clara para frecuencias por encima de $4.8 \mathrm{GHz}$.

Por último, para el canal IB2IB correspondiente a las dos antenas ubicadas en el interior del cuerpo, se ha observado que a diferencia del resto de escenarios de propagación considerados, la función que mejor se ajusta a la forma del espectro Doppler es la normal o gaussiana. Además, debido a la escasa distancia entre antenas y estar ambas inmersas en el phantom, las frecuencias por encima de $4.8 \mathrm{GHz}$ no son practicables debido a la interacción de las antenas en campo cercano lo cual provoca que el nivel de la señal recibido se encuentre bajo el nivel de ruido del sistema. Se ha observado un incremento con la frecuencia en los valores de $B_{D}$ para $3.1 \mathrm{GHz}$ y $4.8 \mathrm{GHz}$, muy similares a los obtenidos mediante el modelo teórico. De igual manera, se ha observado una reducción con la frecuencia en el valor de $T_{c}$ para un nivel de correlación de 0.7 , alcanzando un valor mínimo de 0.22 s a $4.8 \mathrm{GHz}$.

Por último, respecto a los valores para el tiempo de coherencia medidos en los tres escenarios de propagación considerados, estos son muy superiores a los reportados por otros autores considerando la principal causa de variación en la posición relativa entre dispositivos el movimiento de partes del cuerpo. En este sentido, en [D'Errico y Ouvry, 2011] se reportan valores a $2.4 \mathrm{GHz}$ de $T_{c}$ entre $31.2 \mathrm{~ms}$ y $94.75 \mathrm{~ms}$ para el canal on-body, los cuales contrastan con los valores entre 0.22-1.24 s medidos por causa de la respiración. Esto unido a que los periodos de símbolo para cualquiera de las modulaciones contempladas en el estándar IEEE 802.15.6 para la banda de UWB son del orden de ns, se puede concluir que los efectos sobre el canal radio debidos a la variación en la posición relativa entre dispositivos de una WBAN por causa de la respiración son despreciables respecto a los correspondientes a otras fuentes de movimiento. 


\section{Capítulo 5 \\ Conclusiones y Futuras Líneas de Investigación}

En este capítulo se resumen las principales conclusiones obtenidas a partir de los resultados de los estudios presentados en los capítulos anteriores en torno a la caracterización estática y dinámica de la propagación en redes de área corporal inalámbricas (WBAN) en Ultra Wideband. Así mismo, se detallan las posibles líneas de investigación en el campo del modelado del canal de propagación WBAN que podrían desarrollarse más allá del ámbito de la presente tesis. 


\subsection{Conclusiones}

En el capítulo 2 se han presentado los fundamentos teóricos relacionados con las WBAN, los elementos que las componen, las bandas de frecuencias propuestas para establecer los enlaces entre dispositivos y el estado de la estandarización actual, con referente el estándar IEEE 802.15.6. Además, se ha justificado el empleo de la banda UWB en aplicaciones para WBAN debido a las características ofrecidas, como es la posibilidad de dar soporte al desarrollo de aplicaciones que requieran una alta tasa de transferencia o el bajo nivel de potencia radiada lo cual se relaciona con un bajo nivel de tasa de absorción específica (SAR) y alta seguridad en las comunicaciones. Por último, se presentan las características generales de la propagación en la banda UWB aplicada a entornos centrados en el cuerpo y los modelos de canal CM3 y CM4 propuestos en la norma IEEE 802.15.6 para el caso de enlaces on-body y off-body entre dispositivos en esta banda.

En el capítulo 3 se han presentado los estudios correspondientes a la caracterización del canal de propagación off-body entre un dispositivo receptor colocado en determinados puntos sobre la superficie del cuerpo de sujetos reales y un dispositivo transmisor en las inmediaciones actuando como punto de acceso, operando en la banda de frecuencias de 3.1-8 GHz y considerando condiciones de visión directa y estacionalidad en el canal radio. El análisis se ha realizado desde la perspectiva del estudio de la influencia del entorno de medidas, el lugar de colocación sobre el cuerpo de una antena y la postura adoptada por un sujeto. El modelado se ha realizado tanto por medio de la caracterización de las pérdidas de propagación y shadowing (bloqueo de la señal), como por medio del estudio de la dispersión temporal en el canal debido a la propagación multicamino. Además, se ha obtenido el modelo de respuesta al impulso CM4 para UWB propuesto en el estándar IEEE 802.15.6 para dos posturas posibles adoptadas por los sujetos: de pie y tumbados.

Tras los estudios realizados, se puede concluir que la principal causa de variación en las condiciones de propagación en el canal off-body a frecuencias de UWB es la variación del lugar de colocación de un dispositivo sobre el cuerpo de un sujeto. Se ha observado una alta variabilidad y dependencia con las medidas tanto en las pérdidas de propagación como en la dispersión de retardo del canal radio por punto de colocación. Se han medido variaciones de hasta $7.9 \mathrm{~dB}$ en las pérdidas de propagación y hasta 2.61 ns en el valor de dispersión de retardo en enlaces entre un punto de acceso externo y una antena sobre el cuerpo respecto a los valores sin la influencia del cuerpo. Además, considerando la variación en los valores anteriores, se ha encontrado que las posiciones de colocación en la parte superior del cuerpo son las más estables. 
Respecto a la variación en las condiciones de propagación del canal off-body debidas al tamaño del entorno de medidas, se ha observado que aunque al aumentar las dimensiones del entorno un mayor número de componentes llegan con un nivel significativo de potencia al receptor, bajo condiciones de propagación por visión directa cuando un dispositivo está ubicado sobre el cuerpo, debido al efecto de bloqueo de componentes multicamino ejercido por este, la influencia es poco significativa. Así mismo, independientemente del tamaño del entorno, se ha observado un aumento similar de $2 \mathrm{~dB}$ en las perdidas de propagación sobre el cuerpo respecto a condiciones de propagación sin influencia del cuerpo y una disminución del $41 \%$ en el valor de la dispersión de retardo en el canal.

Por último, con respecto a la influencia de la postura en las condiciones de propagación del canal, se ha observado un aumento entre un $46 \%$ y un $53 \%$ en el número de componentes multicamino recibidas por posición de colocación de la antena en el cuerpo para el caso de los sujetos tumbados respecto a la posición de pie. Así mismo, se ha observado que el modelo de canal CM4 propuesto en el estándar IEEE 802.15.6 para UWB se ajusta de manera óptima a las respuestas al impulso obtenidas para ambas posturas consideradas, pudiendo caracterizarse la disminución de la potencia según el retardo mediante una distribución exponencial, el shadowing mediante una distribución normal (o log-normal en el dominio logarítmico) y la distribución del tiempo de llegadas mediante Poisson.

En el capítulo 4 se han presentado los resultados correspondientes a la caracterización en banda estrecha del canal in-body bajo condiciones dinámicas debidas al movimiento relativo entre dispositivos causado por la respiración, considerando cuatro frecuencias englobadas dentro de la banda UWB: $3.1 \mathrm{GHz}, 4.8 \mathrm{GHz}, 6 \mathrm{GHz}$ y $8.5 \mathrm{GHz}$. Se ha considerado un dispositivo en el interior del cuerpo y otro en tres posibles ubicaciones respecto al anterior: sobre el cuerpo, exterior a este a una cierta distancia de la superficie y en el interior del mismo. Esto da lugar a tres posibles canales de propagación: in-body a on-body (IB2OB), in-body a off-body (IB2OFF) e in-body a in-body (IB2IB). Las condiciones de propagación en el interior del cuerpo humano se han simulado por medio de un phantom líquido diseñado para emular las características dieléctricas de los tejidos biológicos con alto contenido en agua en la banda de UWB. El estudio se ha llevado a cabo tanto desde la perspectiva de los efectos en el dominio de la frecuencia, modelando la forma del espectro Doppler y la variación en el ensanchamiento del mismo, como desde la perspectiva de la variación en el tiempo modelada por medio de la función de autocorrelación y el tiempo de coherencia del canal. 
Respecto al modelado de la forma del espectro Doppler, se ha observado que la distribución que mejor se ajusta es la de Laplace tanto en el canal IB2OB como IB2OFF. Por el contrario, la distribución normal o gaussiana es la que mejor ajuste proporciona para la forma del espectro en el canal IB2IB. Debido a la interacción en campo cercano entre las antenas, no se observa a excepción de a $50 \mathrm{~cm}$ para IB2OFF un aumento en el valor del Doppler spread $B_{D}$ con la frecuencia. Además, asociado a esto, al aumentar la frecuencia las pérdidas de propagación introducidas por el medio biológico hacen que el nivel de señal recibido se encuentre muy próximo al nivel de ruido del sistema dejando algunas de las frecuencias impracticables. Esto se observa en especial para el caso del canal IB2IB para frecuencias a partir de $4.8 \mathrm{GHz}$.

En cuanto a la variación temporal en el canal radio asociada al desplazamiento en frecuencia Doppler debido a la respiración, se han obtenido valores para el tiempo de coherencia $T_{c}$ en el rango de $0.22-1.24 \mathrm{~s}$, muy superiores a los reportados por otros autores considerando otras causas para la variación en la posición relativa entre dispositivos de una WBAN. Además, considerando los periodos de símbolo correspondientes a las diferentes modulaciones propuestas en el estándar IEEE 802.15.6 para la banda UWB, se puede concluir que los efectos sobre el canal radio derivados de la respiración son despreciables respecto a los debidos a otras fuentes de movimiento.

\subsection{Futuras líneas de investigación}

El campo de las redes de área corporal inalámbricas no ha dejado de evolucionar desde que se inició la presente tesis. Esto ha ocasionado que, principalmente debido a la futura implantación de los sistemas $5 \mathrm{G}$ en los cuales las WBAN son uno de los elementos clave, la necesidad de caracterización de la propagación en entornos centrados en el cuerpo sea cada vez más importante. Esto tiene como consecuencia que, los estudios presentados aquí puedan ser ampliados cubriendo diferentes aspectos como son los siguientes:

- Estudio del canal estático y dinámico inter-ban o body-body (B2B) entre dispositivos ubicados en la superficie del cuerpo de diferentes sujetos.

- Caracterización de la propagación in-body empleando modelos sintéticos de tejidos heterogéneos y en condiciones reales por medio de medidas en animales.

- Caracterización del canal de propagación desacoplando el efecto de las antenas.

- Modelado de la propagación en la banda de milimétricas, especialmente a $60 \mathrm{GHz}$ y frecuencias superiores (idealmente hasta $\mathrm{THz}$ para futuras redes a nano-escala). 
- Evaluación de técnicas Multiple-Input Multiple-Output (MIMO) en enlaces WBAN UWB con el fin de aumentar la capacidad del canal.

- Desarrollo de materiales multicapa que emulen de manera sintética el comportamiento dieléctrico de los tejidos biológicos a frecuencias tanto de UWB como superiores. 
APÉNDICES 



\section{Modelado del Canal Radio}

A lo largo de los Capítulos 3 y 4 se presentan los resultados correspondientes a la caracterización del canal WBAN considerando en el primero condiciones estáticas y en el segundo dinámicas por el efecto de la respiración. El estudio se ha realizado desde la perspectiva del modelado estadístico del canal a partir de las medidas en frecuencia realizadas empleando como elemento central un analizador de redes vectorial (VNA). En este apéndice se describe la metodología seguida para obtener los parámetros que permiten modelar la propagación en el canal radio tanto en el tiempo como en la frecuencia. En primer lugar se introduce la caracterización en términos de funciones de canal considerando las características de este variables en el tiempo tanto de manera determinista como estocástica. Seguidamente se describen los diferentes tipos de canales habituales y las simplificaciones que pueden derivarse de los mismos sobre las funciones de autocorrelacion del canal. Tras esto se presenta el modelado de las pérdidas de propagación y shadowing, además del efecto de dispersión temporal en el canal debido a la propagación multicamino. Por último se presenta el modelado de los efectos en tiempo y frecuencia derivados del movimiento relativo entre transmisor y receptor.

\section{A.1. Funciones de canal}

El estudio de la propagación de señales en un canal radio puede realizarse desde la perspectiva del análisis de sistemas modelando los efectos en el dominio del tiempo o de la frecuencia producidos por este sobre una señal transmitida. De esta forma, el canal de propagación se puede considerar equivalente a un filtro lineal cuyas características, en general, variarán en el tiempo [Parsons, 2000]. Esta variación puede considerarse tanto determinista como aleatoria, por lo que la salida del canal, vista igualmente como la salida de un filtro lineal, pueden estudiarse por medio de las posibles relaciones tiempo-frecuencia existentes entre entrada y salida. 


\section{A.1.1. Canales deterministas variables en el tiempo}

En el caso de que la variación en el tiempo de las características del canal radio se consideren deterministas, la caracterización de la propagación se puede llevar a cabo por medio de operaciones lineales sobre el siguiente conjunto de funciones que permiten describir el comportamiento del canal en el tiempo y/o la frecuencia [Bello, 1963]:

- $h(t, \tau)$ : Función de ensanchamiento del retardo a la entrada (input delay-spread function).

- $\quad H(f, \rho)$ : Función de ensanchamiento Doppler a la salida (output Doppler-spread function).

- $T(f, t)$ : Función de transferencia variable en el tiempo (time variant transfer function).

- $S(\tau, \rho)$ : Función de ensanchamiento Doppler-retardo (delay Doppler-spread function).

Estas funciones están interrelacionadas por medio de transformadas de Fourier directas $(\mathrm{TF})$ o inversas $\left(\mathrm{TF}^{-1}\right)$. En la Figura A.1 se muestra la relación existente entre las funciones anteriores.

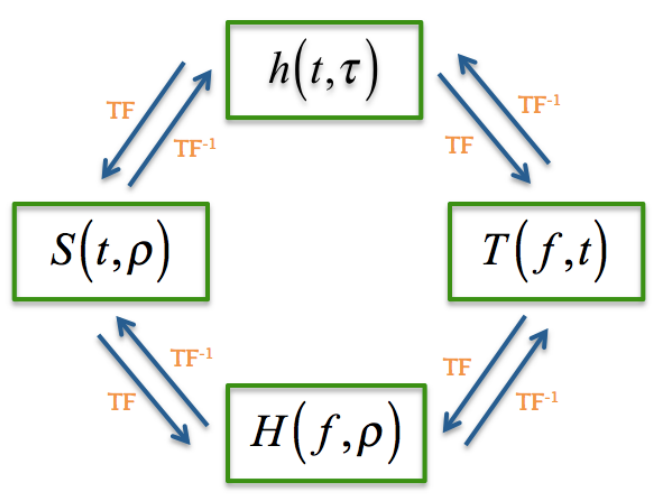

Figura A.1: Relaciones entre funciones de canal.

\section{A.1.2. Canales aleatorios variables en el tiempo}

Habitualmente, la respuesta del canal de propagación no se puede predecir de manera determinista, por lo cual, esta se debe modelar en términos estadísticos. Debido a que, como consecuencia, las funciones descritas anteriormente se convierten en procesos aleatorios, la caracterización completa del canal implicaría el conocer las distribuciones de probabilidad 
conjuntas de todas las funciones anteriores, lo cual es muy complicado en casos reales. Por esto, suponiendo la salida del canal un proceso gausiano (en virtud del teorema del límite central), la descripción del mismo por medio de la media y la autocorrelación se puede considerar estadísticamente completa [Bello, 1963]. De esta forma, las funciones de canal se transforman en las siguientes autocorrelaciones [Parsons, 2000]:

- $\quad E\left[h(t, \tau) h^{*}(s, \eta)\right]=R_{h}(t, s ; \tau, \eta)$

- $\quad E\left[H(f, \rho) H^{*}(m, \mu)\right]=R_{H}(f, m ; \rho, \mu)$

- $\quad E\left[T(f, t) T^{*}(m, s)\right]=R_{T}(f, m ; t, s)$

- $\quad E\left[S(\tau, \rho) S^{*}(\eta, \mu)\right]=R_{S}(\tau, \eta ; \rho, \mu)$

donde por un lado, $t$ y $s$ son variables de tiempo y, $\tau$ y $\eta$ variables de retardo temporal, y por otro, $f$ y $m$ son variables de frecuencia y, $\rho$ у $\mu$ variables de desplazamiento Doppler.

De manera similar al caso de las funciones de canal deterministas, las funciones de autocorrelación anteriores están relacionadas por medio de transformadas de Fourier dobles directas e inversas (DF y $\mathrm{DF}^{-1}$ ). En la Figura A.2 se muestra estas relaciones.

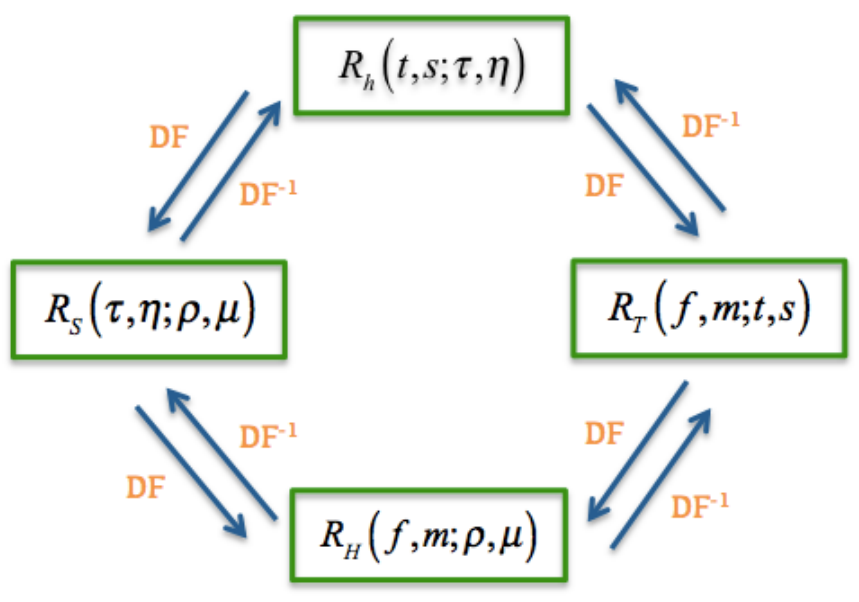

Figura A.2: Relaciones entre funciones de autocorrelación de canal. 


\section{A.1.3. Clasificación de canales habituales. Simplificaciones sobre las funciones de autocorrelación}

Las funciones de autocorrelación de canal descritas arriba pueden simplificarse atendiendo a diferentes características identificadas habitualmente en los canales reales. En general se puede establecer la siguiente clasificación de canales:

- Canales estacionarios en sentido amplio (WSS): en este tipo de canales la estadística del desvanecimiento (fading) puede considerarse, en general, estacionaria para periodos pequeños de tiempo o distancia. Por lo tanto, el valor de la autocorrelación es únicamente dependiente de la diferencia de tiempos y no del instante concreto.

- Canales con scattering incorrelado (US): en estos canales las estadísticas de desvanecimiento correspondientes a contribuciones de dispersores con diferentes reatardos están incorreladas entre sí.

- Canales estacionarios en sentido amplio con scattering incorrelado (WSSUS): son aquellos canales que combinan las características de los canales anteriores. Presentan scatering incorrelado en las variables de retardo temporal y Doppler, y estacionalidad en sentido amplio en las variables de tiempo y frecuencia.

\section{A.2. Caracterización del canal radio}

Asumiendo que el canal radio presenta condiciones de estacionalidad en sentido amplio y scatering incorrelado (WSSUS), es posible realizar el modelado de la propagación en base a las funciones de autocorrelación de canal atendiendo a las propiedades de correlación principalmente en las variables retardo y tiempo [Pahlavan y Levesque, 2005]. De esta forma, la mayor parte de parámetros que nos van a permitir caracterizar el canal radio pueden extraerse de las siguientes dos funciones de autocorrelaciones simplificadas:

$$
\begin{aligned}
& R_{h}(t, s ; \tau, \eta)=R_{h}(\Delta t ; \tau) \delta(\eta-\tau) \triangleq R_{h}(\Delta t ; \tau) \\
& R_{T}(f, m ; t, s)=R_{T}(\Delta f ; t) \delta(s-t) \triangleq R_{T}(\Delta f ; t)
\end{aligned}
$$

En las expresiones anteriores la última simplificación se debe al hecho de considerar condiciones de US (Uncorrelated Scattering) en el canal, por lo que el único valor distinto de cero de la función de autocorrelación se da cuando coinciden los retardos temporales. 
A partir de la expresión (A.1), considerando el canal estático, es decir, cuyas características son independientes de la realización temporal $(\Delta t=0)$, la autocorrelación se convierte en,

$$
R_{h}(\Delta t=0 ; \tau) \Rightarrow P_{h}(\tau)
$$

A $P_{h}(\tau)$ se le denomina perfil de potencia-retardo (PDP) y representa la distribución de la potencia recibida en función del retardo temporal como consecuencia de la transmisión de un impulso en $t=0$.

Por otro lado, considerando en (A.2) una separación en frecuencias de cero $(\Delta f=0)$, la autocorrelación $R_{T}(\Delta f=0 ; t)$ representa la decorrelación de la respuesta al impulso a diferentes instantes temporales cuando se transmite un tono a una única frecuencia. A partir de la transformada de Fourier de esta función de autocorrelación se puede modelar el desplazamiento en frecuencia o efecto Doppler sufrido por una señal debido a la variación de la respuesta del canal en el tiempo.

$$
D(\rho)=\int_{-\infty}^{\infty} R_{T}(\Delta f=0 ; t) \cdot e^{-j 2 \pi \rho t} d t
$$

A $D(\rho)$ se le conoce como espectro de potencia Doppler o Doppler power spectrum.

\section{A.2.1. Modelado estático del canal radio}

Considerando el canal radio estacionario o estático en el tiempo $(\Delta t=0)$, el comportamiento del canal se puede modelar según la expresión (A.3) a partir de un conjunto de parámetros obtenidos por medio del análisis del perfil de potencia-retardo calculado a partir de la respuesta al impulso del canal de la siguiente forma [Rappaport, 1996],

$$
P_{h}(\tau)=|h(\tau)|^{2}
$$

\section{A.2.1.1. Pérdidas de propagación y shadowing}

Las pérdidas de propagación modelan la disminución en la potencia de una señal transmitida debido a la propagación en un cierto medio. Considerando una separación suficiente del transmisor como para considerar campo radiado $\left(R>>2 D^{2} / \lambda\right)$, la reducción 
en el nivel de la señal recibida es proporcional a $1 / d^{2}$, de manera que bajo condiciones de visión directa (LOS) la potencia de la señal recibida puede calcularse de la siguiente forma [Rappaport, 1996],

$$
P_{r}(d)=P_{t} G_{t} G_{r}\left(\frac{\lambda}{4 \pi d}\right)^{2}
$$

donde $P_{t}$ es la potencia transmitida, $G_{t}$ y $G_{r}$ son respectivamente, la ganancia de la antena transmisora y receptora, $\lambda$ es la longitud de onda de la señal transmitida y $d$ es la distancia de separación respecto del transmisor.

De manera general, la potencia media de una señal recibida decrece con el logaritmo de la distancia $d$ con una tasa de reducción modelada por medio de un exponente de pérdidas $n(o \gamma)$. De esta forma, la potencia media de una señal recibida puede expresarse de manera general como,

$$
P L(d B)=P L\left(d_{0}\right)+10 n \log _{10}\left(\frac{d}{d_{0}}\right)
$$

donde $P L\left(d_{0}\right)$ son las pérdidas a una distancia de referencia $d_{0}$ y $n$ es el exponente de pérdidas. En condiciones de propagación en espacio libre $n \approx 2$ lo cual equivale a una reducción de potencia de $20 \mathrm{~dB}$ por década.

Para modelar la variación de las pérdidas de propagación respecto a la media producido por el shadowing u obstrucción del camino directo entre transmisor y receptor, se suele incluir un término adicional a la expresión (A.7) cuya estadística, tal y como se ha reportado en diferentes estudios en WBAN, la distribución estadística que mejor caracteriza al término de shadowing es la normal o gaussiana (log-normal en el dominio logarítmico) [Goswami et al., 2015], teóricamente con media nula y desviación típica $\sigma_{s}\left(N\left(0, \sigma_{s}\right)\right)$. De esta forma, las pérdidas de propagación pueden modelarse de la siguiente forma,

$$
P L(d B)=P L\left(d_{0}\right)+10 n \log _{10}\left(\frac{d}{d_{0}}\right)+S_{\sigma}
$$

De manera experimental, las pérdidas de propagación se pueden modelar a partir del perfil de potencia-retardo del canal, obtenido por medio de la expresión (A.5), de la siguiente forma, 


$$
P L(d B)=10 \log _{10}\left(\frac{1}{\sum_{\tau} P_{h}(\tau)}\right)
$$

\section{A.2.1.2. Dispersión de retardo temporal}

Como consecuencia del efecto multicamino en el canal de propagación, la señal recibida estará formada por múltiples réplicas retardadas y atenuadas de la señal transmitida. Esto produce un aumento en el ensanchamiento efectivo de la señal recibida en el tiempo, y desvanecimientos en frecuencia.

A partir del perfil de potencia-retardo del canal se puede cuantificar el efecto de dispersión de retardo en base a dos parámetros principales: retardo medio o $\tau_{m}$ (mean excess delay) y valor cuadrático medio (rms) del ensanchamiento o $\tau_{r m s}$. Estos parámetros son respectivamente el primer momento central y la raíz cuadrada del segundo momento central del PDP y se pueden calcular de la siguiente forma [Rappaport, 1996],

$$
\begin{gathered}
\tau_{m}=\frac{\sum_{k=1}^{L} P_{h}\left(\tau_{k}\right) \cdot \tau_{k}}{\sum_{k=1}^{L} P_{h}\left(\tau_{k}\right)} \\
\tau_{r m s}=\sqrt{\frac{\sum_{k=1}^{L} P_{h}\left(\tau_{k}\right) \cdot \tau_{k}^{2}}{\sum_{k=1}^{L} P_{h}\left(\tau_{k}\right)}-\left(\frac{\sum_{k=1}^{L} P_{h}\left(\tau_{k}\right) \cdot \tau_{k}}{\sum_{k=1}^{L} P_{h}\left(\tau_{k}\right)}\right)^{2}}
\end{gathered}
$$

Debido a que diferentes canales con perfiles de potencia-retardo distintos pueden dar lugar al mismo retardo medio $\tau_{m}$, habitualmente se emplea el parámetro $\tau_{r m s}$ para cuantificar el efecto de la dispersión de retardo en el canal.

\section{A.2.1.3. Ancho de banda de coherencia}

Como se ha indicado en la sección anterior, el efecto en frecuencia derivado del ensanchamiento de la señal recibida como consecuencia de la propagación multicamino, es el aumento del desvanecimiento en frecuencia del canal. Se puede definir el ancho de banda de coherencia $B_{c}$ como el rango de frecuencias para las cuales el canal presenta una atenuación plana (igual para todas las frecuencias). De esta forma, si el ancho de banda de la señal 
transmitida $B_{s} \ll B_{c}$, el desvanecimiento sufrido será plano, mientras que si sucede lo contrario, el desvanecimiento será selectivo en frecuencia, es decir, unas frecuencias sufrirán más desvanecimiento que otras. Esto es equivalente a decir que una señal sufrirá desvanecimiento plano en frecuencia si el periodo de símbolo $T_{s} \gg \tau_{r m s}$ (habitualmente $\left.T_{s} \geq 10 \tau_{r m s}\right)$.

Debido a la relación inversa entre tiempo y frecuencia, el ancho de banda de coherencia del canal y la dispersión de retardo se pueden relacionar de la siguiente forma,

$$
B_{c} \approx \frac{1}{k \cdot \tau_{r m s}}
$$

donde $k=5$ para un nivel de correlación en frecuencia de 0.5 y $k=50$ para un nivel de correlación de 0.9 [Rappaport, 1996].

\section{A.2.2. Modelado dinámico del canal radio}

Como consecuencia del movimiento relativo entre una antena transmisora (Tx) y una receptora $(\mathrm{Rx})$ o de los elementos en el canal, las diferentes componentes recibidas llegarán al receptor con diferencias de fase variables con el tiempo. De esta manera, considerando una señal transmitida sin modular $s(t)=\Re\left\{e^{j\left(2 \pi f_{c} t+\phi_{0}\right)}\right\}$, la señal recibida será de la forma:

$$
r(t)=\Re\left\{\sum_{i=1}^{L} \beta_{i}(t) \cdot e^{-j\left(2 \pi f_{c}\left(t-\tau_{i}(t)\right)+\phi_{0}\right)}\right\}
$$

donde $\tau_{i}(t)$ es el retardo temporal correspondiente a la componente multicamino i-ésima recibida, el cual dependerá de la longitud del camino recorrido y de la velocidad $v$ de desplazamiento de Tx hacia Rx. En el caso de que el medio de transmisión no sea el aire $\left(\varepsilon_{r}=1\right)$, la velocidad de propagación en el medio ya no será $c \approx 3 \cdot 10^{8}$ si no que vendrá determinada por la permitividad relativa $\varepsilon_{r}$ del medio para la frecuencia considerada. De manera simplificada, considerando una única componente recibida (LOS), el retardo sufrido por esta debido al efecto del medio de propagación será,

$$
\tau_{\text {medio }} \approx \frac{d_{0}-v t}{c / \sqrt{\left|\varepsilon_{r}\right|}}
$$


Derivando la fase de $r(t)$ respecto al tiempo obtenemos la frecuencia instantánea. De esta forma,

$$
\frac{\partial \phi(t)}{\partial t}=f_{c} \pm \frac{f_{c} v}{c / \sqrt{\left|\varepsilon_{r}\right|}}
$$

Es decir, la frecuencia de la señal recibida en función del tiempo sufrirá una variación o efecto Doppler que dependerá de la velocidad relativa entre Tx y Rx.

La caracterización del desplazamiento Doppler que sufre una señal puede realizarse a partir del espectro de potencia Doppler calculado por medio de la expresión (A.4). De manera práctica, este puede obtenerse a partir de la esperanza $E\left\{H^{*}\left(f_{c}, \Delta t\right) \cdot H\left(f_{c}, t+\Delta t\right)\right\}$. Alternativamente, la función de autocorrelación $R_{T}(\Delta f=0 ; t)$ se puede expresar a partir de la operación: $\quad R_{T}\left(f_{c} ; \Delta t\right)=H\left(f_{c}, \Delta t\right) \otimes H^{*}\left(f_{c},-\Delta t\right) \quad$ donde $\otimes \quad$ es $\quad$ el operador convolución. Tomando la transformada de Fourier de la autocorrelación anterior, la convolución se transforma en una multiplicación, por lo tanto, el espectro Doppler considerando una frecuencia de transmisión $f_{c}$, se puede calcular como: $D\left(f_{c}, \rho\right)=H\left(f_{c}, \rho\right) \cdot H^{*}\left(f_{c},-\rho\right)$ y debido a la simetría real de $H\left(f_{c}, \rho\right)$, se obtiene que: $D\left(f_{c}, \rho\right)=\left|H\left(f_{c}, \rho\right)\right|^{2}$. Por lo tanto, ya que la función $H\left(f_{c}, t\right)$ corresponde con el parámetro de dispersión $S_{21}$ del canal medido por medio del VNA, la función $D\left(f_{c}, \rho\right)$ se puede obtener a partir del módulo al cuadrado de la transformada de Fourier de la respuesta en frecuencia del canal para la frecuencia $f_{c}$ de la siguiente forma,

$$
D\left(f_{c}, \rho\right)=\left|T F\left\{H\left(f_{c}, t\right)\right\}\right|^{2}
$$

\section{A.2.2.1. Dispersión Doppler}

A partir de la función $D\left(f_{c}, \rho\right)$ obtenida por medio de la expresión (A.16) se pueden derivar dos parámetros que permiten modelar el carácter dispersivo en frecuencia del canal de propagación. Estos parámetros son el ensanchamiento Doppler $B_{D}$, definido como el rango de frecuencias para el cual el espectro Doppler es superior a un cierto umbral y el 
ensanchamiento Doppler rms, calculado como la raíz cuadrada del segundo momento central de la función $D\left(f_{c}, \rho\right)$ de la siguiente forma,

$$
\rho_{r m s}=\sqrt[2]{\frac{\int \rho^{2} D\left(f_{c}, \rho\right) d \rho}{\int D\left(f_{c}, \rho\right) d \rho}}
$$

\section{A.2.2.2. Tiempo de coherencia del canal}

Por medio de la función de autocorrelación (ACF, Autocorrelation Function), obtenida a partir de la respuesta en frecuencia $H\left(f_{c}, t\right)$ del canal, se puede realizar un análisis de la variación temporal del mismo debida al movimiento relativo entre Tx y Rx. Podemos calcular la función de autocorrelación para una frecuencia de operación $f_{c}$ de la siguiente forma.

$$
R_{T}\left(f_{c} ; t\right)=E\left\{H\left(f_{c}, t\right) \cdot H^{*}\left(f_{c}, t\right)\right\}
$$

donde $H\left(f_{c}, t\right)$ es la respuesta en frecuencia del canal formada por cada uno de los valores medidos por medio del VNA para $N$ instantes temporales, obtenida como:

$$
H\left(f_{c}, t\right)=\left\{H\left(f_{c}, t_{1}\right), H\left(f_{c}, t_{2}\right), \ldots, H\left(f_{c}, t_{N}\right)\right\}
$$

A partir de la función de autocorrelación $R_{T}\left(f_{c} ; t\right)$, se puede obtener el tiempo de coherencia del canal $T_{c}$ definido como el intervalo de tiempo durante el cual la amplitud de dos señales presentan un cierto grado de coherencia [Rappaport, 1996]. Visto de otra manera, es el tiempo durante el cual la respuesta al impulso del canal es invariante. En general, el grado de coherencia que se suele considerar para el cálculo varía de 0.5 a 0.9 . Al igual que ancho de banda de coherencia y el ensanchamiento de retardo guardan una relación inversa, $T_{c}$ puede obtenerse a partir del desplazamiento Doppler del canal, de la siguiente forma,

$$
T_{c}=\frac{k}{f_{m}}
$$

donde $f_{m}$ es el máximo desplazamiento Doppler $\left(f_{m} \approx v f_{c} / c\right)$. Si se considera un valor de correlación superior a $0.5, k$ puede fijarse a 0.423 [Kumar, 2015]. 
En el caso de que el periodo de la señal transmitida $T_{s}<<T_{c}$, el canal se mantendrá estable durante el tiempo de transmisión de cada símbolo. Por lo tanto, el canal presentará desvanecimiento lento. Esto es equivalente a considerar que el ancho de banda de la señal transmitida $B_{s}$ es inferior al valor del Doppler spread $\left(B_{s}<B_{D}\right)$. Si por el contrario $T_{s}>T_{c}, 0$ $B_{s} \gg B_{D}$, el canal variará durante el tiempo de transmisión dando lugar a desvanecimiento rápido.

\section{A.2.3. Otras estadísticas de segundo orden}

Además de los parámetros descritos en las secciones anteriores, los cuales permiten realizar una caracterización del canal en términos de dispersión temporal o frecuencial, en ocasiones es de utilidad el empleo de estadísticas de segundo orden para obtener una visión más completa del canal radio bajo condiciones dinámicas. Dentro de este tipo de estadísticas, las más empleadas son:

- Tasa de cruce por nivel (LCR, Level Crossing Rate).

- Duración media del desvanecimiento (AFD, Average Fading Duration).

La tasa de cruce por nivel permite determinar, para un determinado nivel de potencia de recepción, el número de veces por segundo que la señal recibida cruza ese nivel en sentido ascendente o descendente.

Por otro lado, la duración del desvanecimiento se define como el tiempo medio que la envolvente de la señal recibida permanece bajo un determinado nivel de potencia y permite determinar el tiempo medio durante el cual la señal sufre un determinado nivel de desvanecimiento.

Dado que, en diferentes estudios la distribución estadística que mejor se ajusta al desvanecimiento en los canales BAN es la log-normal [Taparugssanagorn et al., 2009], los valores teóricos de $\operatorname{LCR}\left(N_{R_{d B}}\right)$ y $\operatorname{AFD}\left(T_{R_{d B}}\right)$ pueden obtenerse de la siguiente forma [Cotton y Scanlon, 2007],

$$
\begin{gathered}
N_{R_{d B}}\left(r_{d B}\right)=f_{m} \cdot \exp \left(-\frac{\left(r_{d B}-\mu_{d B}\right)^{2}}{2 \sigma_{d B}^{2}}\right) \\
T_{R_{d B}}\left(r_{d B}\right)=\frac{N_{R_{d B}}^{-1}\left(r_{d B}\right)}{2}\left(1+e r f\left[\frac{r_{d B}-\mu_{d B}}{\sigma_{d B} \sqrt{2}}\right]\right)
\end{gathered}
$$


donde $f_{m}=v \cdot f_{c} / c$ es el máximo desplazamiento Doppler a la frecuencia $f_{c}, \mu$ y $\sigma$ son respectivamente la media y la desviación típica de la envolvente de la señal recibida y erf $[\cdot]$ es la función de error, la cual puede estimarse de la siguiente forma

$$
\operatorname{erf}[u]=\frac{2}{\sqrt{\pi}} \sum_{n=0}^{\infty} \frac{(-1)^{n} u^{2 n+1}}{(2 n+1) n !}
$$

\section{A.2.4. Metodología de modelado del canal de propagación}

A la hora de realizar la caracterización del canal de propagación con el fin de plantear un modelo del mismo se puede optar por dos enfoques básicos: determinista y estadístico (o analíticos).

\section{A.2.4.1. Modelado determinista}

El modelado determinista del canal radio se basa en la caracterización detallada de todos los elementos que influyen en la propagación en un escenario concreto (geometría del entorno, posición de Tx y Rx, propiedades electromagnéticas de los materiales, etc.) y en la aplicación de técnicas de análisis electromagnético, tales como el método de los elementos finitos (FEM, Finite Elements Method), el método de las diferencias finitas en el dominio del tiempo (FDTD, Finite Difference Time Domain), el método de los momentos (MoM, Method of Moments), trazado/lanzado de rayos, etc., con el objetivo de resolver las ecuaciones de Maxwell teniendo en cuenta todas las condiciones de contorno especificadas.

Con el fin de caracterizar la propagación en el cuerpo humano por medio de simulaciones electromagnéticas, habitualmente se emplean modelos numéricos (phantoms numéricos), habitualmente restringidos a una cierta parte del cuerpo. Estos pueden variar desde modelos geométricos simplificados a modelos detallados en los que se definen múltiples tipologías de tejidos, cada uno de ellos con sus propiedades dieléctricas. Para más detalles acerca de la propagación en el cuerpo y phantoms, se puede consultar el Apéndice B, sección B.1.1 de la presente tesis.

Debido a los altos requerimientos computaciones que los métodos deterministas conllevan, sobre todo conforme aumenta el área analizada y la frecuencia, su empleo se suele centrar en el modelado de la propagación en pequeñas áreas del cuerpo, usualmente empleando simplificaciones según el tipo de escenario de propagación bajo estudio (in-body, on-body u off-body) y la frecuencia de operación. De esta forma, se pueden emplear modelos 
unicapa (homogéneos) en escenarios on-body u off-body a frecuencias de UWB debido a que a esas frecuencias la propagación se produce en la superficie del cuerpo y no a través de él, o modelos multicapa (heterogéneos) para simular la propagación en el interior del cuerpo teniendo en cuenta los diferentes tejidos involucrados. Además, debido a la alta dependencia de los resultados con el escenario analizado, las conclusiones obtenidas por medio de los métodos deterministas son difícilmente extrapolables a otros escenarios de propagación (otros entornos, partes del cuerpo, diferentes frecuencias, etc.).

\section{A.2.4.2. Modelado estadístico}

El modelado estadístico del canal se basa en la caracterización de los diferentes factores que influyen en la propagación de señales por medio de variables aleatorias caracterizadas por medio de sus distribuciones de probabilidad. Los diferentes modelos de propagación son, por tanto, formulados en base a expresiones matemáticas cuyos parámetros se modelan a partir de las observaciones derivadas de medidas experimentales del canal de propagación real. A diferencia de los métodos deterministas, el ámbito de aplicación de las expresiones que describen la propagación en el canal radio no está reducido a las condiciones en las cuales se obtuvo el modelo sino que pueden extrapolarse, pudiéndose adaptar a distintos escenarios. Esto es de gran utilidad ya que, en condiciones reales, la posición del transmisor y receptor varía en el tiempo así como la posición de los elementos dispersores (scatters) entre ambos, haciendo los modelos deterministas poco viables en estos casos.

El proceso central a partir del cual se plantea un modelo estadístico de la propagación de señales en un canal WBAN lo constituye la medida (channel sounding) del mismo, llevado a cabo sobre sujetos reales o sobre phantoms físicos diseñados para emular las propiedades dieléctricas de los tejidos humanos correspondientes a una cierta parte del cuerpo (ver el Apéndice B, sección B.1.2), siendo estos los más empleados a la hora de realizar medidas destinadas a la caracterización del canal WBAN in-body.

Las medidas del canal radio pueden realizarse en dos dominios: en tiempo y en frecuencia.

\section{A. Medidas del canal en el tiempo}

Las medidas del canal de propagación en el dominio del tiempo se basan en el empleo como señal transmitida de un pulso de anchura temporal $\tau_{b b}$ muy reducida, o un tren de estos, y la obtención de la respuesta al impulso $h(t, \tau)$ del canal empleando un osciloscopio digital. Tanto el ancho y forma del pulso como el periodo de repetición deben 
escogerse adecuadamente de manera que se pueda caracterizar el comportamiento variable en el tiempo del canal con la resolución requerida, así como asegurarse de que todas las posibles réplicas de la señal transmitida hayan sido recibidas antes de emitir el siguiente pulso. Hay que tener en cuenta que la resolución de las medidas, es decir, la capacidad de determinar componentes multicamino individuales viene limitado por el ancho de banda del pulso transmitido $\left(1 / \tau_{b b}\right)$. En la Figura A.3. se puede ver un esquema simplificado de un sistema de medidas del canal radio en el tiempo.

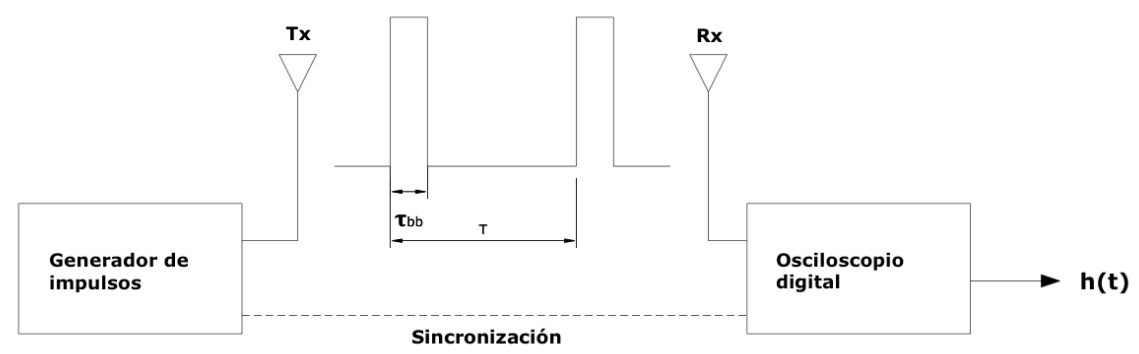

Figura A.3: Esquema de medidas del canal radio en el tiempo.

La señal recibida por el osciloscopio será por lo tanto $r(t)=x(t) \otimes h(t, \tau)$, es decir, la convolución entre la señal transmitida y la respuesta al impulso del canal. Con el fin de poder obtener $h(t, \tau)$ es necesario por lo tanto disponer en recepción de una réplica de la señal transmitida, de manera que aplicando la convolución inversa es posible obtener la respuesta al impulso del canal. Por este motivo, cuanto más sencillo sea el pulso empleado en transmisión más sencillo será el proceso de obtención de la respuesta del canal.

La principal ventaja de las técnicas de medidas del canal radio en el tiempo es que este puede variar durante el intervalo de tiempo en el cual se realiza la medida, por lo cual es posible realizar una caracterización el comportamiento variable temporal del mismo. Sin embargo, en su aplicación a UWB, presenta la desventaja de la generación de impulsos de duración tan pequeña y el alto grado de sincronización necesario.

\section{B. Medidas del canal en frecuencia}

Las medidas en el dominio de la frecuencia se basan en el barrido por medio de una señal sinusoidal de un rango de frecuencias de interés y la medición de la respuesta en frecuencia del canal $H(f, t)$ por medio de un analizador redes vectorial (VNA). Usualmente el analizador de redes actúa tanto como generador de la señal de barrido como de receptor por lo cual se pueden obtener medidas con un alto rango dinámico realizando las configuración de ancho de banda intermedio (IF, Intermediate Frequency) y potencia transmitida requeridas. 
La respuesta en frecuencia del canal viene caracterizada por el parámetro de dispersión $S_{21}$ medido por medio del VNA y la resolución temporal de la medida se puede aproximar por la inversa del ancho de banda $(\Delta \tau \approx 1 / B W)$.

Una vez obtenida la respuesta en frecuencia, es posible obtener la respuesta al impulso del canal realizando la transformada inversa de Fourier (IFT, Inverse Fourier Transform). Previo a realizar la IFT, las muestras de la respuesta en frecuencia son multiplicadas por una función ventana (Rectangular, Hann, Hamming, etc.) con el fin de reducir los errores por truncamiento debidos al hecho de tener una respuesta en frecuencia finita. Esto tiene como resultado un mejora en el nivel de los lóbulos secundarios de la respuesta al impulso pero conlleva una reducción en la resolución temporal proporcional a las características de la ventana empleada [Wang y Wang, 2013].

Ya que con únicamente un equipo es suficiente para realizar las medidas, es el método más empleado para la banda de UWB. La principal desventaja de este método es que, debido al alto tiempo requerido por el barrido en frecuencia y a que el canal debe permanecer estático durante ese tiempo (el tiempo de barrido debe ser inferior al tiempo de coherencia del canal), no es posible caracterizar el comportamiento variable en el tiempo del canal. Además de manera general, el método de medidas en frecuencia está limitado por el máximo retardo detectable, limitado superiormente por la siguiente expresión:

$$
\tau_{\max }=\frac{N_{\text {samp }}-1}{B W}
$$

donde $N_{s m p}$ es el número de muestras en frecuencia realizadas y $B W$ es el ancho de banda total medido. En la Figura A.4. se muestra un esquema simplificado de este tipo de técnicas de medida del canal de propagación.

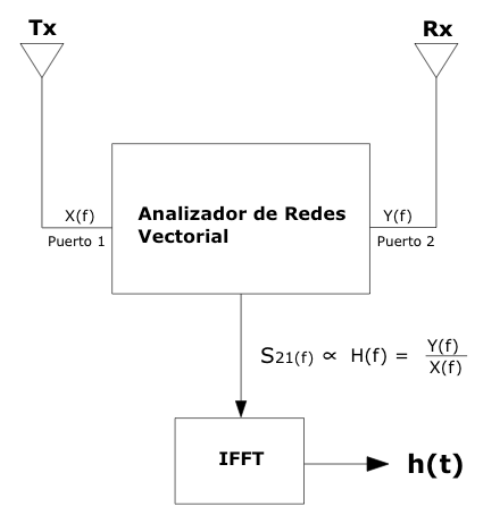

Figura A.4: Esquema de medidas del canal radio en frecuencia. 


\section{Fundamentos de la Propagación en Tejidos Biológicos}

El cuerpo humano es quizás la estructura biológica más compleja existente. De manera general, está formado por multitud de tejidos, constituidos estos por agrupaciones organizadas de células con niveles de especialización similares. Los tejidos, a su vez, son los elementos constitutivos de los órganos. El estudio de la propagación de señales a través del cuerpo, por lo tanto, supone el estudio de la interacción entre una onda electromagnética y el cuerpo a nivel tanto celular como de los tejidos.

Este apéndice presenta una descripción de los fundamentos teóricos de la propagación en tejidos biológicos por medio de la caracterización de la variación en los parámetros dieléctricos (permitividad relativa y conductividad) en función de la frecuencia. Así mismo, se presentan modelos, tanto numéricos como sintéticos, que permiten emular el comportamiento de los tejidos biológicos, con el propósito de caracterizar la propagación de señales radio en el cuerpo sin la necesidad de emplear tejidos reales.

\section{B.1. Propagación en tejidos biológicos}

El estudio del comportamiento del cuerpo ante la exposición a una onda electromagnética se fundamenta en el fenómeno de la polarización (reorientación de los dipolos respecto al campo incidente) a nivel celular. Para esto, es importante distinguir las partes de las células implicadas. De esta forma podemos distinguir tres elementos:

- Fluido intracelular.

- Membrana celular.

- Fluido extracelular.

Cada uno de los elementos anteriores posee unas características eléctricas en cuanto resistividad y capacitancia cuya variación depende de, además del tipo de células, de la frecuencia de operación. Por otro lado, las células se unen entre sí formando tejidos a través 
del fluido extracelular, de manera que dependiendo de las características de esta unión, se puede hacer la siguiente clasificación de los mismos:

- Tejidos con alto contenido en agua (tejido muscular, sangre, etc.).

- Tejidos con bajo contenido en agua (tejido óseo, piel, etc.).

Un caso particular de la clasificación anterior es el tejido adiposo, cuyas características dependen de la localización de este tipo de tejido en el cuerpo, siendo el nivel de concentración de agua mayor o menor dependiente de esta.

La forma habitual de modelar las características dieléctricas de los tejidos humanos es por medio del análisis de la variación con la frecuencia de la permitividad compleja $\left(\dot{\varepsilon}_{r}(\omega)=\varepsilon_{0}\left(\varepsilon_{r}^{\prime}-j \varepsilon_{r}^{\prime \prime}\right)\right)$ de estos. Esta variación no es uniforme y para cada tejido se puede identificar una serie de zonas de dispersión a lo largo de su espectro dieléctrico, cada una caracterizada por un mecanismo de interacción principal entre el campo electromagnético y el tejido según la frecuencia [Wang y Wang, 2013]. De esta manera, a bajas frecuencias (zona $\alpha$ ), la dispersión en la permitividad compleja esta asociada a los procesos de difusión iónica en la membrana celular. Para frecuencias intermedias por bajo de $\mathrm{GHz}$ (zona $\beta$ ) la dispersión está asociada, entre otros fenómenos, a la polarización de la membrana celular (conducción de corriente a través de esta). Por último, a frecuencias de GHz (zona $\gamma$ ) la dispersión está asociada a la polarización de las moléculas de agua. En la Figura B.1 se muestra cada una las zonas de dispersión identificadas.

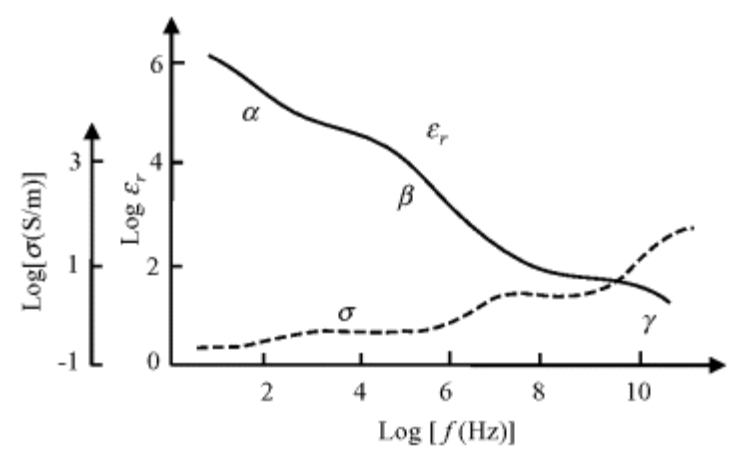

Figura B.1: Zonas de dispersión en el espectro dieléctrico para tejidos biológicos [Wang y Wang, 2013].

En la Figura B.1 se puede observar adicionalmente, la relación inversa con la frecuencia entre la permitividad relativa y la conductividad para los tejidos. La variación con la frecuencia de la permitividad relativa, para cada una de las zonas anteriores puede modelarse a partir de una expresión de Debye como la siguiente, 


$$
\dot{\varepsilon}_{r}(\omega)=\varepsilon_{\infty}+\frac{\Delta \varepsilon}{1+j \omega \tau}
$$

donde $\tau$ es el tiempo de relajación, definido como el retardo experimentado por un material dieléctrico en polarizarse por la acción de un campo electromagnético incidente, por la anchura de la zona $\Delta \varepsilon$ y por el valor de la permitividad a frecuencias de terahercios $\varepsilon_{\infty}$ $(\omega \tau \gg 1)$. A partir de esta expresión, el comportamiento dieléctrico de los tejidos humanos se ha observado que puede modelarse por medio de cuatro zonas de dispersión ( $\alpha, \beta, \gamma$ más una zona en alta frecuencia) por medio de la expresión conocida como 4 Cole-Cole [Cole y Cole, 1941],

$$
\dot{\varepsilon}_{r}(\omega)=\varepsilon_{\infty}+\sum_{n=1}^{4} \frac{\Delta \varepsilon_{n}}{1+\left(j \omega \tau_{n}\right)^{1-\alpha_{n}}}+\frac{\sigma_{0}}{j \omega \varepsilon_{0}}
$$

Esta expresión constituye una generalización semiempírica de la expresión de dispersión de Debye, válida para frecuencias hasta $10 \mathrm{GHz}$ [Wang y Wang, 2013]. A diferencia de la expresión de Debye, se incluye un parámetro $\alpha_{n}$ que modela la anchura de la zona de dispersión, ajustándose mejor al comportamiento observado. Adicionalmente, se incluye el término $\sigma_{0}$ mediante el cual se modela la corriente de conducción iónica a frecuencia $0 \mathrm{~Hz}$. Aunque esta expresión permite modelar de manera precisa la variación con la frecuencia de la permitividad relativa compleja para los tejidos, su utilización es complicada, principalmente mediante simulaciones electromagnéticas, por lo cual se suelen emplear simplificaciones de la misma, eliminando unos términos u otros en función de la banda de frecuencias bajo estudio [Khaleghi et al., 2010].

Con el fin de realizar el modelado de la dependencia con la frecuencia de la permitividad compleja de lo tejidos biológicos, a partir de medidas experimentales realizadas sobre muestras reales de animales (principalmente ovejas) y material forense, Gabriel et al. realizaron un extenso trabajo de caracterización en base a las expresiones Cole-Cole que, hasta el momento, constituye la base de referencia más completa sobre caracterización de las propiedades dieléctricas en tejidos biológicos. En [Gabriel y Gabriel, 1996] se presentan los resultados de la caracterización anterior sobre los tejidos más comunes, relativa al rango de frecuencias de $10 \mathrm{~Hz}$ a $20 \mathrm{GHz}$. En la Tabla B.1 se muestra un ejemplo particularizado a un conjunto de tejidos y órganos a $5 \mathrm{GHz}$. 


\begin{tabular}{|c|c|c|c|}
\hline Tejido/Órgano & $\begin{array}{c}\text { Conductividad } \\
\text { (S/m) }\end{array}$ & $\begin{array}{c}\text { Permitividad } \\
\text { relativa }\end{array}$ & $\begin{array}{c}\text { Tangente de } \\
\text { pérdidas }\end{array}$ \\
\hline Piel seca & 3.06 & 35.77 & 0.31 \\
\hline Piel húmeda & 3.57 & 39.61 & 0.32 \\
\hline Grasa & 0.24 & 5.03 & 0.17 \\
\hline Músculo & 4.04 & 49.54 & 0.29 \\
\hline Hueso esponjoso & 1.81 & 16.05 & 0.41 \\
\hline Hueso cortical & 0.96 & 10.04 & 0.34 \\
\hline Médula ósea & 0.23 & 5.04 & 0.17 \\
\hline Materia gris cerebral & 4.10 & 45.15 & 0.33 \\
\hline Materia blanca cerebral & 2.86 & 33.44 & 0.31 \\
\hline Humor vítreo & 5.41 & 65.81 & 0.30 \\
\hline Sangre & 5.40 & 53.95 & 0.36 \\
\hline Corazón & 4.86 & 50.27 & 0.35 \\
\hline Riñón & 4.94 & 48.06 & 0.37 \\
\hline Hígado & 3.83 & 39.26 & 0.35 \\
\hline Pulmón hinchado & 3.94 & 44.86 & 0.32 \\
\hline Pulmón deshinchado & 1.72 & 18.97 & 0.33 \\
\hline Esófago & 5.16 & 57.89 & 0.32 \\
\hline Estómago & 5.16 & 57.89 & 0.32 \\
\hline Colon & 4.58 & 49.72 & 0.33 \\
\hline Duodeno & 5.16 & 57.89 & 0.32 \\
\hline Intestino delgado & 5.75 & 49.98 & 0.41 \\
\hline
\end{tabular}

Tabla B.1: Propiedades dieléctrica de los principales tejidos y órganos a $5 \mathrm{GHz}$.

A partir de los modelos desarrollados por Gabriel et al. es posible obtener tanto la permitividad relativa como la conductividad según la frecuencia para la mayoría de tejidos biológicos existentes. Esto constituye un elemento indispensable en los estudios tanto de dosimetría para el calculo de la tasa de absorción específica (SAR) como del modelado de las pérdidas de propagación en redes WBAN. Una aplicación de los modelos de Gabriel et al. es la disponible a través desde la web del Instituto de Física Aplicada (IFAC) de Florencia en [IFAC-CNR].

\section{B.2. Emulación de tejidos. Phantoms}

El modelado de la interacción del cuerpo humano con una señal electromagnética es en general complejo. Debido a que en ocasiones no es posible llevar a cabo la caracterización de la propagación sobre sujetos reales, por las dificultades técnicas o éticas que implican, habitualmente se emplean modelos denominados phantoms, que simulan las características de los tejidos biológicos en un cierto rango de frecuencias. Estos proporcionan además, un entorno de medidas estable, controlable y repetible, condiciones difícilmente alcanzables en medidas sobre sujetos reales [Wang y Wang, 2013]. Aunque se pueden realizar diversas 
clasificaciones para la tipología de phantoms (según contenido en agua, rango de frecuencias, etc.) la más habitual es la siguiente en base a la naturaleza de los modelos:

- Modelos numéricos o virtuales (phantoms numéricos).

- Modelos sintéticos (phantoms sintéticos).

○ Líquidos.

○ Semisólidos.

○ Sólidos.

En las siguientes secciones se detallan las características de cada uno de los tipos de phantoms listados.

\section{B.2.1. Modelos numéricos (phantoms numéricos)}

Los modelos numéricos, o phantoms numéricos, son modelos anatómicos virtuales o modelos geométricos simplificados diseñados para la caracterización de la propagación en unas condiciones específicas por medio de un software de modelado electromagnético. Los modelos anatómicos virtuales se forman a partir de imágenes médicas obtenidas mediante imágenes por resonancia magnética (MRI) o tomografía axial computarizada (TAC), que posteriormente son segmentadas y transformadas en estructuras compuestas por píxeles volumétricos 3D (voxels) de una determinada resolución y unas características dieléctricas concretas que emulan a las del tejido humano al cual se asocian. Los modelos obtenidos por este método pueden exportarse a un software de análisis electromagnético y ser empleados en las simulaciones. Existen diferentes modelos disponibles, tanto libres (normalmente obtenidos por medio de la voxelización de imágenes médicas obtenidas de la Visible Human Project ${ }^{\circledR}$ de la National Library of Medicine) como comerciales como los de la Virtual Population de la fundación IT'IS, los cuales son una extensión de los modelos desarrollados para la Virtual Family [Christ et al, 2010], algunos de los cuales se muestran en la Figura B.2.

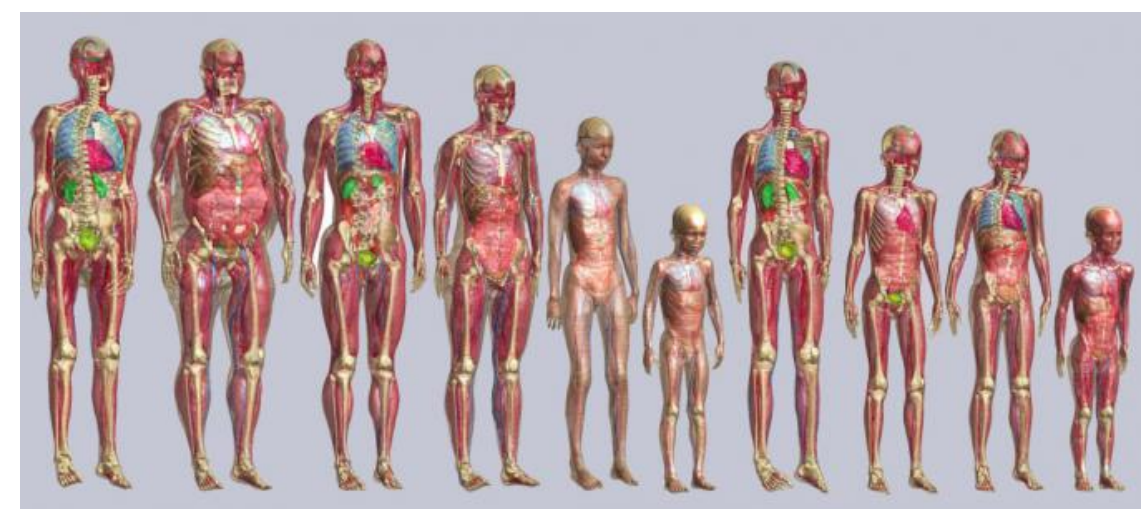

Figura B.2: Modelos numéricos de la Virtual Population [IT’IS Foundation] 
En ocasiones es interesante incluir la componente dinámica en las simulaciones, para lo cual se emplean diferentes técnicas entre las que están la captura de movimiento (Motion Capture) [Hoeckel et al., 2015], [Swaisaenyakorn et al., 2012] o las aplicaciones de animación 3D como Poser $^{\odot}$ combinadas con herramientas de modelado electromagnético. Esto permite aplicar como entrada a la simulación cada uno de los fotogramas generados de forma que, ajustando el nivel de detalle requerido y el número de fotogramas es posible obtener resultados de simulaciones teniendo en cuenta determinadas dinámicas.

\section{B.2.2. Modelos sintéticos (phantoms sintéticos)}

Aunque los modelos numéricos descritos anteriormente permiten incluir en las simulaciones ciertos aspectos de la interacción de señales electromagnéticas con los tejidos biológicos de manera teórica, no son capaces de proporcionar resultados realistas derivados de un entorno sujeto a interferencias electrónicas, mecánicas y ambientales [Mobashsher y Abbosh, 2015]. Con el objetivo de poder caracterizar los efectos sobre los tejidos biológicos en ámbitos como la dosimetría, hipertermia, imagen por microondas, implantes, propagación en WBAN, etc., de manera repetible y sin la necesidad de realizar medidas sobre sujetos reales, es posible emplear diferentes materiales diseñados con el fin de emular las características de los tejidos biológicos en una cierta banda de frecuencias. A estos materiales se les denominan phantoms de tejidos sintéticos (synthetic tissue phantoms) o phantoms sintéticos.

Dependiendo del resultado final del proceso de fabricación, este tipo de phantoms se pueden clasificar en líquidos, semisólidos y sólidos [Hall y Hao, 2012].

\section{B.2.2.1. Phantoms líquidos}

Este tipo de phantoms están fabricados en base a soluciones líquidas con una determinada formulación la cual posee unas ciertas características dieléctricas, similares a las de los tejidos biológicos que se presenten simular, dentro de una determinada banda de frecuencias. Los compuestos que principalmente se emplean para el diseño de este tipo de phantoms son agua desionizada, azúcar (para controlar la permitividad relativa) y cloruro de sodio (para controlar la conductividad). Otros compuestos como el hidroxietilcelulosa se suelen emplear para controlar la permitividad y la conductividad [Wang y Wang, 2013] mediante gelificación. Por su naturaleza, este tipo de phantoms son los que permiten emular de manera más precisa los tejidos con alto contenido en agua, como son los músculos, aunque la emulación de tejidos con bajo contenido en agua (como el tejido óseo y algunos tipos de 
tejido adiposo) es posible a partir de emulsiones de agua y diferentes tipos de aceites (parafina, aceites vegetales, etc.) [Mobashsher y Abbosh, 2015].

Este tipo de phantoms tienen la ventaja de ser sencillos de fabricar y permiten caracterizar de manera precisa la distribución de campo en el interior del medio biológico. Por el contrario, suponen un medio de propagación homogéneo por lo que el comportamiento en entornos de reales heterogéneos compuestos por múltiples capas de tejidos puede no ser el modelado. Además, al ser soluciones líquidas, debe emplearse un contenedor que presente un comportamiento dieléctrico caracterizado por una baja permitividad relativa y conductividad [Hall y Hao, 2012]. Por último, este tipo de phantoms no son adecuados para la caracterización de la tasa de absorción específica (SAR) en la superficie.

A pesar de que este tipo de phantoms han sido empleados extensivamente en el análisis de la propagación en WBAN a frecuencias de la banda ISM, hay pocos estudios en los cuales se empleen formulaciones válidas para frecuencias superiores, como las de la banda UWB. En este sentido, el phantom propuesto en [Zhou et al., 2006], empleado en la presente tesis durante las medidas para la caracterización dinámica del canal radio considerando al menos uno dispositivo de la red WBAN en el interior del cuerpo, supone uno de los pocos diseños destinados a ser empleados en el modelado de la propagación en la banda de UWB. Otros ejemplos de phantoms líquidos validos para UWB y frecuencias superiores son los presentados en [Palacios et al., 2016] diseñados para emular entre 3-18 GHz las características dieléctricas de diferentes tipos de tejidos humanos.

\section{B.2.2.2. Phantoms semisólidos}

Los phantoms de tipo semisólidos son variaciones de los anteriores a los cuales se les ha añadido un agente gelatinizante, como es el agar, el TX-151 o la glicerina. Para controlar las propiedades dieléctricas se suele emplear polvo de poliestireno (permitividad) y cloruro sódico (conductividad) [Hall y Hao, 2012], [Wang y Wang, 2013]. En general, el proceso se hace dentro de un molde, de manera que el resultado final es una estructura con un alto contenido en agua con una forma determinada. Por lo tanto, este tipo de phantoms son adecuados para la emulación de estructuras multicapa de tejidos biológicos blandos en los cuales las capas de tejido poseen una determinada forma o patrón [Mobashsher y Abbosh, 2015].

Uno de los principales problemas de este tipo de phantoms es su rápida degradación con el tiempo, por lo que son difícilmente reusables o almacenables durante un largo periodo 
de tiempo. Se han reportado duraciones de cerca de un mes para phantoms semisólidos con base de TX-151 recubiertos de vinilo y hasta seis meses con glicerina [Wang y Wang, 2013].

Aunque de manera similar a lo que sucede con los phantoms líquidos, el número de estudios en la literatura sobre el diseño de materiales semisólidos para emular tejidos biológicos a frecuencias de UWB o superiores es reducido, esta es la tipología de phantoms más extendida. Algunos ejemplos de phantoms semisólidos son los presentados en [Yilmaz et al., 2014] para emular el comportamiento dieléctrico de la piel mojada, tejido adiposo, sangre y músculo en la banda de $0.3-20 \mathrm{GHz}$, los descritos en [Ishido et al., 2004] para cuerpo y cabeza en la banda de 3-6 GHz, o los diseñados para aplicaciones de imagen por microondas para la detección de cáncer de mama descritos en [Bakar et al., 2011] y [Baskharoun et al., 2012] para la banda de UWB de 3-11 GHz y 3-10 GHz respectivamente.

\section{B.2.2.3. Phantoms sólidos}

Este tipo de phantoms están fabricados por medio de estructuras capaces de mantener la forma durante un largo periodo de tiempo y son especialmente adecuados para la caracterización de la propagación alrededor del cuerpo o en su interior debido a que pueden emular de manera precisa estructuras multicapa no homogéneas [Hall y Hao, 2012]. Aunque, al igual que en los casos anteriores, pueden tener diferentes composiciones, en general suelen están fabricados a partir de diferentes mezclas de materiales tales como cerámica y grafito, silicona con fibra de carbono, plástico conductivo con carbón negro, etc. [Hall y Hao, 2012]. Debido a la complejidad que conlleva la fabricación de este tipo de phantoms, tienen un precio superior a los de los tipos anteriores [Mobashsher y Abbosh, 2015], aunque al degradarse más lentamente, la vida útil es muy superior comparada con la de los phantoms líquidos o semisólidos. Suelen tener partes desmontables y pueden a su vez rellenarse con otros materiales, según el efecto a investigar.

Al contrario de los phantoms líquidos o semisólidos, los cuales tienen una base de agua, el control de las características dieléctricas en los phantoms sólidos es complejo, por lo que obtener materiales con una buen comportamiento en una banda ancha de frecuencias es, en general, difícil. Un ejemplo de phantom sólido multicapa es el presentado en [Garrett y Fear, 2015], fabricado en base a una mezcla de grafito, carbón negro y uretano, desarrollado para aplicaciones de imagen por microondas para la detección de cáncer de mama en la banda de $10 \mathrm{MHz}$ a $12 \mathrm{GHz}$. Adicionalmente, en [Guraliuc et al., 2014] se presenta un phantom sólido fabricado en base a una mezcla de carbón negro y polidimetilsiloxano para emular el comportamiento de la piel humana a $60 \mathrm{GHz}$. 


\section{Hardware y Software para Medidas del Canal Radio}

El proceso de medidas del canal de propagación puede llevarse a cabo tanto en el dominio del tiempo como de la frecuencia. En el caso de la caracterización en el dominio del tiempo, el objetivo final es la obtención de respuesta al impulso $h(\tau)$ del canal radio. Por el contrario, el objetivo de la caracterización en el dominio de la frecuencia es la obtención de la respuesta en frecuencia $H\left(f_{c}\right)$. A partir de la relación existente entre ambos dominios, ambas respuestas están relacionadas por medio de una transformada de Fourier (directa o inversa),

$$
\begin{gathered}
h(\tau)=\int_{-\infty}^{\infty} H(f) e^{j 2 \pi f \tau} d f \\
H(f)=\int_{-\infty}^{\infty} h(\tau) e^{-j 2 \pi f \tau} d \tau
\end{gathered}
$$

En las expresiones anteriores se asume que el canal de propagación es estático, por lo tanto las respuestas son invariantes en el tiempo. En el caso habitual, la respuesta del canal varía en el tiempo debido bien al movimiento de Tx y/o Rx o bien debido al movimiento de los elementos reflectores, difractores o dispersores presentes en el canal. Por esta razón, ambas respuestas se modelan como procesos estocásticos $h(t, \tau)$ y $H(f, t)$ en los cuales, cada instante de medida $t$ del canal da lugar a una realización temporal del proceso.

Debido a la mayor simplicidad a la hora de obtener una caracterización del canal de propagación, el método de medidas en frecuencia es habitualmente el más empleado en el estudio de la propagación radio en entornos centrados en el cuerpo como son las redes de área corporal inalámbricas, y por lo tanto es el método elegido durante las campañas de medida realizadas que han servido de base durante la presente tesis. Por lo tanto, el objetivo de este apéndice es la descripción de los diferentes elementos hardware y software empleados para la caracterización en el dominio de la frecuencia del canal de propagación WBAN. 


\section{C.1. Analizador de redes vectorial}

El elemento central que posibilita la medida de la respuesta en frecuencia del canal de propagación es el analizador de redes vectorial (VNA). Durante las campañas de medidas desarrolladas a lo largo de la presente tesis se emplearon dos equipos del fabricante Agilent: ENA E5071B y ENA E5072A, tal y como se pueden ver en la Figura B.1.

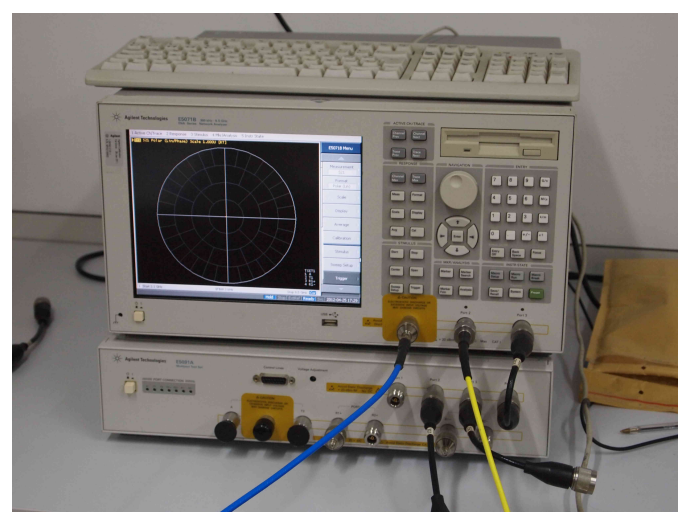

(a)

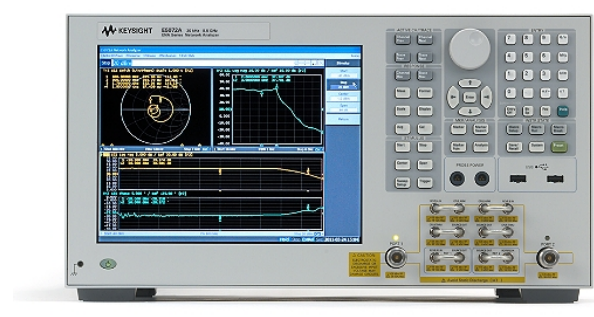

(b)

Figura C.1: Analizadores de redes empleados en las medidas. (a) Agilent ENA E5071B, (b) Agilent ENA E5072A.

Ambos analizadores permiten realizar medidas hasta los $8.5 \mathrm{GHz}$, por lo tanto la banda de frecuencias efectiva en UWB se acotó de 3.1-8.5 GHz. Debido a que únicamente se emplearon dos antenas en las medidas (sistema SISO), el puerto 1 se utilizó para transmisión y el puerto 2 para recepción.

Con el fin de eliminar la influencia de los cables, conectores y amplificador en las medidas, previo a cada cambio de disposición de los elementos o configuración particular del analizador se realizó un calibrado del sistema. El tipo de calibrado empleado fue del tipo Response(Thru). En este tipo de calibración se conecta el cable que conectaría con la antena Tx con el que iría a la antena Rx de manera que queda cerrado el circuito entre el puerto de Tx del analizador (puerto 1) y el de Rx (puerto 2). De esta manera se pueden compensar las pérdidas y desplazamientos en fase ocasionadas por los elementos presentes en el sistema medida a excepción de las antenas.

\section{C.2. Antenas}

Para las medidas se emplearon tres tipos de antenas, todas ellas con una banda de operación dentro de la banda de UWB de $3.1 \mathrm{GHz}$ a $8.5 \mathrm{~Hz}$ considerada: EM-6116, Johanson 3100AT51A7200 y antena UWB iTEAM. 


\section{A. Antena EM-6116}

La antena EM-6116 posee un patrón de radiación omni-direccional y $1 \mathrm{dBi}$ de ganancia. Esta antena puede trabajar en la banda de frecuencias de 2-10 GHz, cubriendo casi al completo la banda de UWB (3.1-10.6 GHz). Esta antena únicamente se empleó como transmisora. En la Figura C.2. se puede ver una imagen de esta antena junto con el diagrama de las pérdidas de retorno medidas para esta.

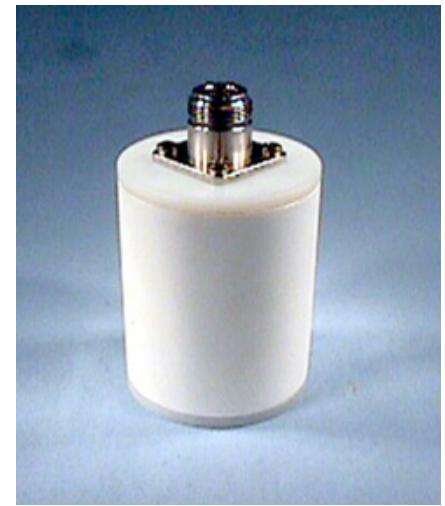

(a)

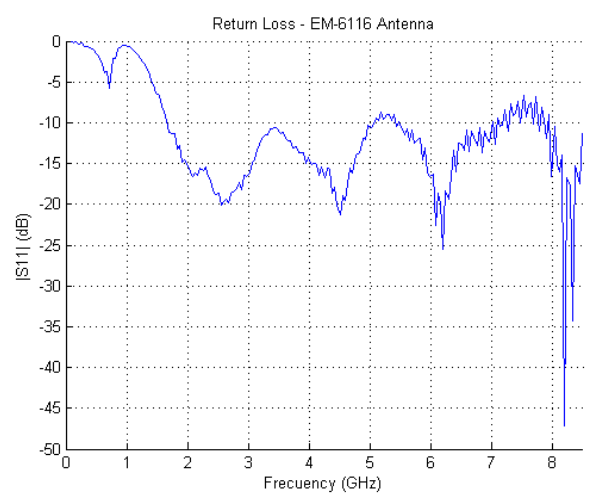

(b)

Figura C.2: Antena EM-6116. (a) Imagen de la antena, (b )pérdidas de retorno.

B. Antena Johanson Tech. 3100AT51A7200

La antena 3100AT51A7200 del fabricante Johanson Technology se encuentra montada sobre una PCB de 54x70 mm y por motivos de fiabilidad nos fue suministrada por el propio fabricante. La antena presenta un formato de chip conteniendo un monopolo sobre un substrato cerámico. Es una de las pocas antenas de bajo perfil actuales que es capaz de trabajar en la banda completa de UWB (3.1-10.3 GHz) y posee unas pérdidas de retorno para la banda de trabajo inferiores a $-10 \mathrm{~dB}$. Esta antena tiene una ganancia media de $-3.5 \mathrm{dBi}$ y una ganancia de pico de $1.5 \mathrm{dBi}$. En la Figura C.3. se puede ver una imagen de esta antena junto con el diagrama de las pérdidas de retorno medidas para esta. 


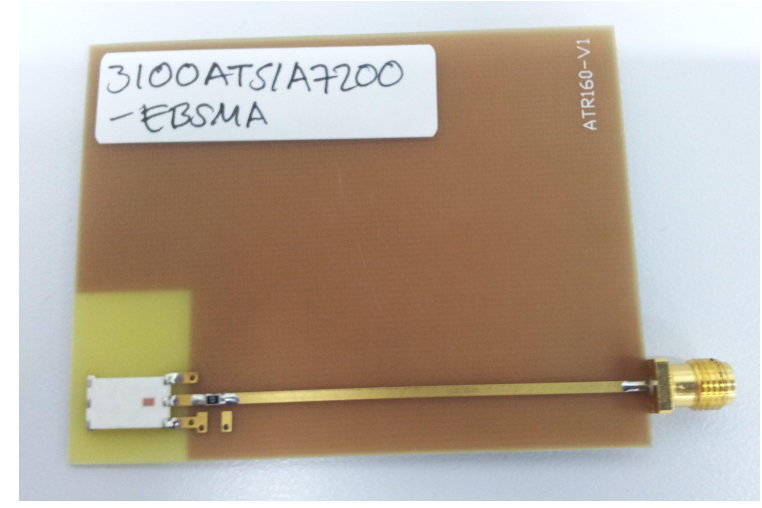

(a)

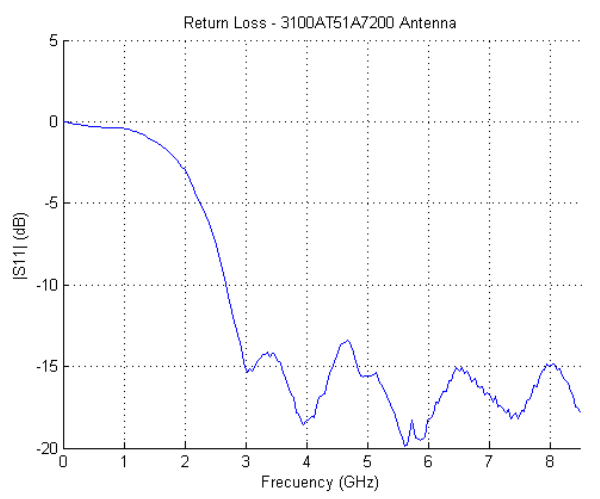

(b)

Figura C.3: Antena Johanson Tech. 3100AT51A7200. (a) Imagen de la antena, (b) pérdidas de retorno.

\section{Antena UWB iTEAM}

Se trata de una antena de tipo parche de tamaño $5 \times 4.4 \mathrm{~cm}$ desarrollada por parte del iTEAM de la UPV. Esta antena se diseño para operar en la banda de UWB. En la Figura C.4. se puede ver una imagen de esta antena junto con las pérdidas de retorno (simuladas y medidas).

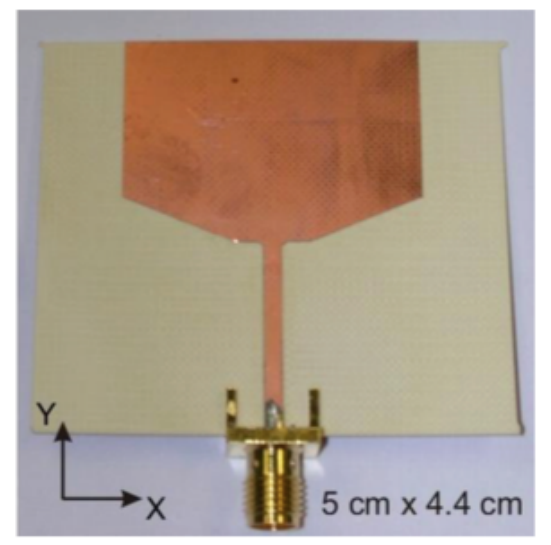

(a)

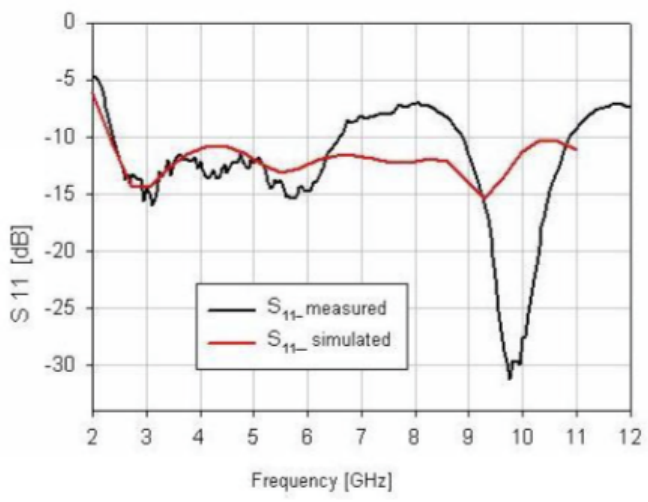

(b)

Figura C.4: Antena UWB iTEAM. (a) Imagen de la antena, (b) pérdidas de retorno.

\section{C.3. Amplificador}

Con el fin de aumentar el nivel de la señal transmitida y mejorar el rango dinámico de las medidas, se empleó un amplificador Mini-Circuits ZVE-8G con una ganancia para la banda de 3.1-8 GHz de $34 \mathrm{~dB}$ a una tensión de alimentación de $12 \mathrm{~V}$. 


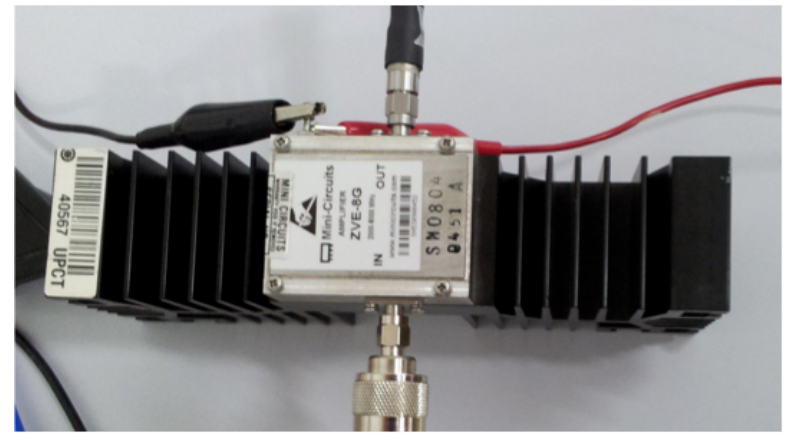

(a)

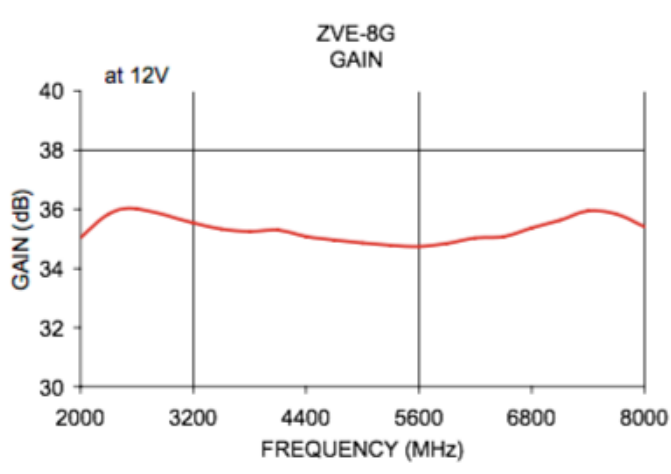

(b)

Figura C.5: Amplificador Mini-Circuits ZVE-8G. (a) Imagen del amplificador, (b) respuesta en frecuencia.

\section{C.4. Fuente de alimentación}

Con el fin de alimentar los amplificadores, se empleó la fuente de alimentación variable modelo EP613-A. Esta fuente puede proporcionar una tensión de salida entre 0 y 24 $\mathrm{V}$, con una corriente alterna de hasta 3 A. En la Figura C.6. se muestra una imagen de la fuente empleada.

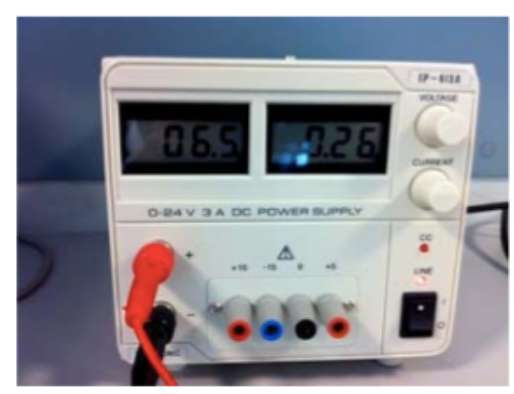

Figura C.6: Fuente de alimentación EP613-A.

\section{C.5. Cables}

Para la interconexión de los diferentes elementos empleados se usaron dos tipos de cables coaxiales: cables de $1.5 \mathrm{~m}$ con unas pérdidas de $2 \mathrm{~dB}$ y cable de $6 \mathrm{~m}$ con unas pérdidas de $10 \mathrm{~dB}$.

\section{C.6. Phantom UWB}

En las medidas para la caracterización del canal BAN cuando uno de los dispositivos se encuentra implantado en el interior del cuerpo se empleó un phantom líquido diseñado para simular la permitividad relativa compleja $\dot{\varepsilon}=\varepsilon^{\prime}-j \varepsilon^{\prime \prime}$ de los tejidos humanos con alto 
contenido en agua (como los músculos) a las frecuencias de UWB de $3.1 \mathrm{GHz}$ a $10.6 \mathrm{GHz}$. Este phantom fue formulado en base a una solución de sacarosa $\left(\mathrm{C}_{12} \mathrm{H}_{22} \mathrm{O}_{11}, 1.0 \mathrm{~mol} / \mathrm{l}\right)$ tal y como se describe en [Zhou et al., 2006]. La parte real de la permitividad compleja $\varepsilon^{\prime}$ dentro de la banda de operación presenta una variación entre 30 y 50 para una temperatura de referencia de $25^{\circ} \mathrm{C}$. En la Figura C.7 se muestra la variación en la parte real y compleja del phantom para el rango de frecuencias de operación.

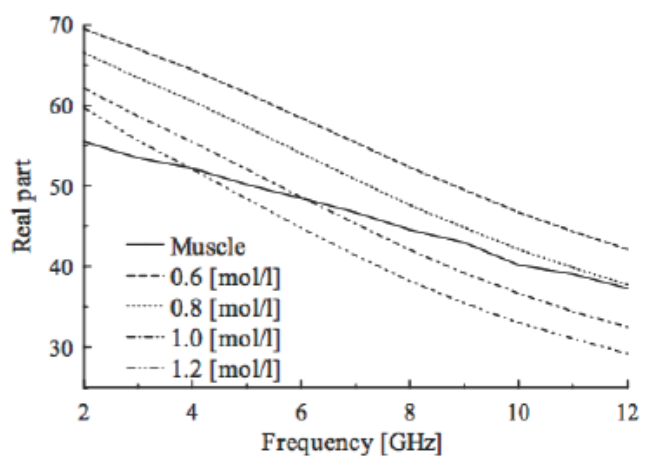

(a)

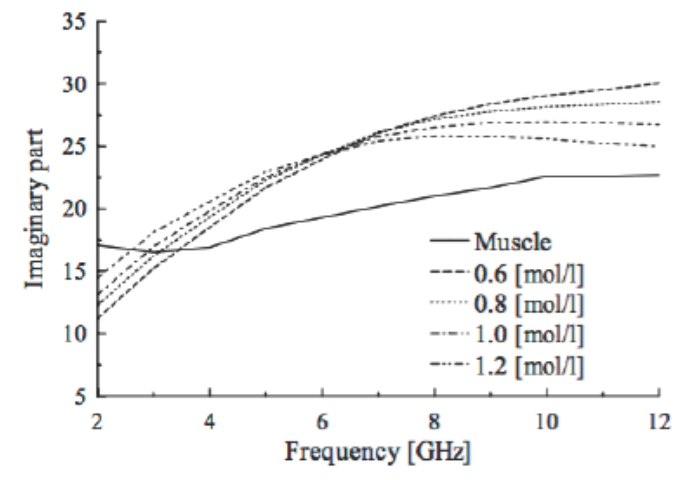

(b)

Figura C.7: Permitividad relativa compleja del phantom empleado en comparación con la del tejido muscular. (a) Parte real, (b) parte imaginaria. [Zhou et al., 2006].

\section{C.7. Software controlador}

El control del equipo ENA E5071B se realizó por medio de una aplicación previamente desarrollada por el departamento de Sistemas de Comunicaciones Móviles ( $\mathrm{SiCoMo}$ ) de la UPCT, instalada en un ordenador portátil conectado a su vez con el VNA por medio de un bus GPIB (General-Purpose Interface Bus) empleando el interfaz de conexión Agilent 8235A USB/GPIB. En la figura C.8. se muestra el aspecto gráfico de esta aplicación.
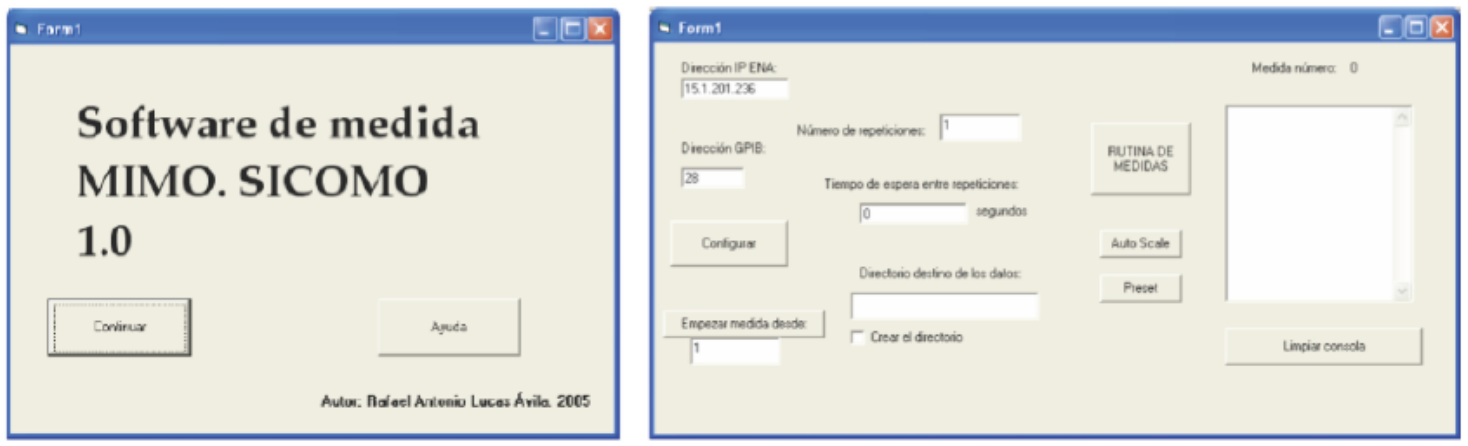

Figura C.8: Aplicación controladora para ENA E5071B. 
Esta aplicación permite por un lado generar una configuración para el proceso de medidas y enviarla al VNA y por otro lado lanzar el proceso de medidas configurando, además, el número de repeticiones de la medida, el tiempo entre el inicio de cada medida y el directorio de almacenamiento de los archivos.

Los ficheros generados y almacenados en el directorio del VNA configurado por medio de esta aplicación, contienen información de módulo y fase para cada uno de los pasos en frecuencia indicados (1601 de $3.1 \mathrm{GHz}$ a $10 \mathrm{GHz}$ ). Esta información corresponde con el muestreo de la respuesta en frecuencia del (parámetro $\mathrm{S}_{21}$ ) canal medido. Por lo tanto, a partir de esta información, se puede recomponer la respuesta en frecuencia compleja $H(f)$.Un ejemplo del formato de estos ficheros es el mostrado en la Figura C.9.

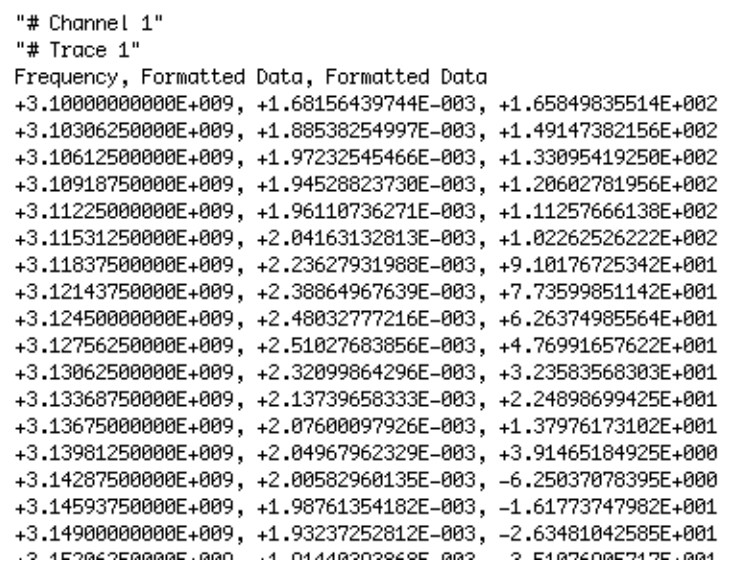

Figura C.9: Ejemplo de fichero de datos generado por el VNA.

Con el fin de automatizar y controlar las medidas realizadas mediante el equipo ENA E5072A se empleó una aplicación desarrollada en Matlab por parte del iTEAM de la UPV funcionando en un ordenador portátil conectado al analizador por medio de una conexión LAN mediante Ethernet. 


\section{LISTA DE FIGURAS}

Figura 1.1: Distribución de la población en Europa según edad media [UN, 2015]..................1

Figura 2.1: Niveles de interconexión en WBAN [Movassaghi et al., 2014].............................8

Figura 2.2: Topologías de red contempladas en el estándar IEEE 802.15.6. (a) Topología en estrella, (b) topología en estrella extendida [IEEE 802.15.6, 2012] .........................................15

Figura 2.3: Modos de acceso en IEEE 802.15.6. (a) Con baliza y delimitadores de trama, (b) sin baliza y delimitadores de trama, (c) sin baliza ni delimitadores de trama [Ullah et al., 2013].

Figura 2.4: Estructura de PPDU para PHY NB [IEEE 802.15.6, 2012] ................................18

Figura 2.5: Estructura de PPDU para PHY HBC [IEEE 802.15.6, 2012]..............................19

Figura 2.6: Estructura de PPDU para PHY UWB [IEEE 802.15.6, 2012] .............................21

Figura 2.7: Estructura de seguridad en el estándar IEEE 802.15.6. ....................................22

Figura 2.8: Mascaras de emisión genéricas UWB. (a) ETSI [ETSI TR 103 181-1, 2015], (b)

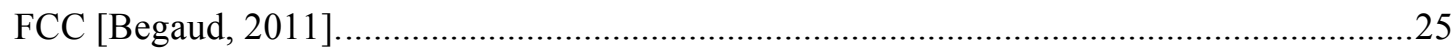

Figura 2.9: Canales de propagación en BAN [IEEE P802.15-08-0780-09-0006, 2009] ........28

Figura 2.10: Propagación multicamino en WBAN.

Figura 2.11: Dependencia de las pérdidas de propagación según la frecuencia en tejidos con alto contenido en agua [Wang y Wang, 2013].

Figura 2.12: Modelo de respuesta al impulso variable temporal en un canal multicamino

[Rappaport, 1996].

Figura 2.13: Modelo de respuesta al impulso para el canal CM3 en el estándar IEEE 802.15.6.

Figura 3.1: Esquema de medidas en frecuencia del canal off-body UWB..............................43

Figura 3.2: Medición del rango dinámico del sistema de medidas. ...........................................44

Figura 3.3: Plano de la planta sótano del edificio de la ETSIT.................................................46

Figura 3.4: Esquema del entorno de medidas en el laboratorio TSC5 ……............................46 
Figura 3.5: Esquema del entorno de medidas en el laboratorio del SiCoMo. .47

Figura 3.6: Posiciones de colocación de la antena Rx sobre el cuerpo de los sujetos 48

Figura 3.7: Montaje para medidas del canal sin presencia de un sujeto. (a) escenario general de medidas, (b) detalle de la antena receptora.

Figura 3.8: PDPs análisis de influencia del entorno de medidas. (a) Sin sujeto en laboratorio TSC5, (b) sin sujeto en laboratorio del SiCoMo, (c) con sujeto en laboratorio TSC5, (d) con sujeto en laboratorio del SiCoMo.

Figura 3.9: Variación de las pérdidas de camino respecto del entorno de medidas en medidas con y sin cuerpo. (a) TSC5, (b) SiCoMo. 54

Figura 3.10: Variación del ensanchamiento de retardo del canal por entorno de medidas. (a) Sin sujeto, (b) con antena en sujeto.

Figura 3.11: Entorno de medidas y posiciones de colocación de la antena Rx sobre el cuerpo.

Figura 3.12: Valor medio de pérdidas de propagación por cada enlace en el cuerpo respecto a los valores de referencia sin cuerpo. .60

Figura 3.13: Variación media en el rms delay spread para cada posición de Rx en el cuerpo con sujetos a $2 \mathrm{~m}$ respecto al valor sin unfluencia del cuerpo.

Figura 3.14: Esquema de medidas con sujetos de pie (a) y tumbados (b).......

Figura 3.15: Mesa para medidas con sujetos tumbados.

Figura 3.16: APDP normalizados para cada postura de los sujetos.

Figura 3.17. Ajuste lineal de los perfiles de potencia-retardo.

Figura 3.18: Ajuste estadístico del parámetro $\beta$. (a) función de distribución para sujetos de pie, (b) función de probabilidad acumulada para sujetos de pie, (c) función de distribución para sujetos tumbados, (d) función de probabilidad acumulada para sujetos tumbados.

Figura 3.19: Ajuste estadístico del tiempo entre llegadas según modelo de Poisson. (a) función de distribución para sujetos de pie, (b) función de probabilidad acumulada para sujetos de pie, (c) función de distribución para sujetos tumbados, (d) función de probabilidad acumulada para sujetos tumbados.

Figura 4.1: Esquema general de elementos para medidas en frecuencia del canal in-body dinámico debido al efecto de la respiración. 
Figura 4.3: Respuesta en frecuencia del filtro de media móvil para $L=20$.

Figura 4.4: Reducción del ruido presente en la respuesta en frecuencia medida. (a) Respuesta

original, (b) respuesta filtrada. 84

Figura 4.5: Efecto del filtrado sobre el espectro Doppler. 84

Figura 4.6: Esquema de elementos para medidas del canal dinámico IB2OB. .86

Figura 4.7: Esquema de elementos para medidas del canal dinámico IB2OFF. .86

Figura 4.8: Esquema de elementos para medidas del canal dinámico IB2IB. 86

Figura 4.9: Variación con la frecuencia de la potencia media recibida (parámetro A) para IB2IB, IB2OB y IB2OFF $(10 \mathrm{~cm}, 20 \mathrm{~cm}, 30 \mathrm{~cm}$ y $50 \mathrm{~cm})$.

Figura 4.10: Ajuste de $\left|H\left(f_{c}, t\right)\right|$ al modelo teórico para el canal IB2OB. (a) $3.1 \mathrm{GHz}$, (b) 4.8

$\mathrm{GHz}$, (c) $6 \mathrm{GHz}$ y (d) $8.5 \mathrm{GHz}$.

Figura 4.11: Ajuste de $H\left(f_{c}, t\right)$ al modelo teórico para el canal IB2OFF para $10 \mathrm{~cm}$ entre Tx y Rx. (a) $3.1 \mathrm{GHz}$, (b) $4.8 \mathrm{GHz}$, (c) $6 \mathrm{GHz}$ y (d) $8.5 \mathrm{GHz}$. 91

Figura 4.12: Ajuste de $H\left(f_{c}, t\right)$ al modelo teórico para el canal IB2OFF para $50 \mathrm{~cm}$ entre Tx y

Rx. (a) $3.1 \mathrm{GHz}$, (b) $4.8 \mathrm{GHz}$, (c) $6 \mathrm{GHz}$ y (d) $8.5 \mathrm{GHz}$. .92

Figura 4.13: Variación en la potencia media recibida (parámetro A) según distancia para el canal IB2OFF.

Figura 4.14: Ajuste de $H\left(f_{c}, t\right)$ al modelo teórico para el canal IB2IB. (a) $3.1 \mathrm{GHz}$, (b) 4.8

$\mathrm{GHz}$, (c) $6 \mathrm{GHz}$ y (d) $8.5 \mathrm{GHz}$. .94

Figura 4.15: Normalización en amplitud del espectro Doppler. (a) Espectro sin normalizar, (b) espectro normalizado a $0 \mathrm{~dB}$.

Figura 4.16: Ajuste de la forma del espectro Doppler para el canal IB2OB a $3.1 \mathrm{GHz}$.

Figura 4.17: Valores de RMSE para el ajuste de la forma del espectro Doppler en el canal IB2OFF 100

Figura 4.18: Ajuste de la forma del espectro Doppler para el canal IB2IB a $3.1 \mathrm{GHz}$. 101

Figura 4.19: Modelo teórico de proceso respiratorio. 102

Figura 4.20: Espectros Doppler para el canal IB2OB. (a) $3.1 \mathrm{GHz}$, (b) $4.8 \mathrm{GHz}$, (c) $6 \mathrm{GHz}$ y (d) $8.5 \mathrm{GHz}$. 103 
Figura 4.21: Espectros Doppler para el canal IB2OFF a $10 \mathrm{~cm}$. (a) $3.1 \mathrm{GHz}$, (b) $4.8 \mathrm{GHz}$, (c)

$6 \mathrm{GHz}$ y (d) $8.5 \mathrm{GHz}$. 105

Figura 4.22: Espectros Doppler para el canal IB2OFF a $50 \mathrm{~cm}$. (a) $3.1 \mathrm{GHz}$, (b) $4.8 \mathrm{GHz}$, (c)

$6 \mathrm{GHz}$ y (d) $8.5 \mathrm{GHz}$. 105

Figura 4.23: Variación del Doppler spread con la distancia en el canal IB2OFF. 107

Figura 4.24: Espectros Doppler para el canal IB2IB. (a) $3.1 \mathrm{GHz}$, (b) $4.8 \mathrm{GHz}$, (c) $6 \mathrm{GHz}$ y

(d) $8.5 \mathrm{GHz}$

Figura 4.25: Función de autocorrelación del proceso respiratorio real y ajuste exponencial.110

Figura 4.26: Variación del tiempo de coherencia con la distancia en el canal IB2OFF. 112

Figura A.1: Relaciones entre funciones de canal. 126

Figura A.2: Relaciones entre funciones de autocorrelación de canal. 127

Figura A.3: Esquema de medidas del canal radio en el tiempo 138

Figura A.4: Esquema de medidas del canal radio en frecuencia.

Figura B.1: Zonas de dispersión en el espectro dieléctrico para tejidos biológicos [Wang y Wang, 2013].

Figura B.2: Modelos numéricos de la Virtual Population [IT'IS Foundation]

Figura C.1: Analizadores de redes empleados en las medidas. (a) Agilent ENA E5071B, (b) Agilent ENA E5072A.

Figura C.2: Antena EM-6116. (a) Imagen de la antena, (b )pérdidas de retorno.

Figura C.3: Antena Johanson Tech. 3100AT51A7200. (a) Imagen de la antena, (b) pérdidas de retorno.

Figura C.4: Antena UWB iTEAM. (a) Imagen de la antena, (b) pérdidas de retorno. 152

Figura C.5: Amplificador Mini-Circuits ZVE-8G. (a) Imagen del amplificador, (b) respuesta en frecuencia. 153

Figura C.6: Fuente de alimentación EP613-A. 153

Figura C.7: Permitividad relativa compleja del phantom empleado en comparación con la del tejido muscular. (a) Parte real, (b) parte imaginaria. [Zhou et al., 2006]. 154

Figura C.8: Aplicación controladora para ENA E5071B. 154

Figura C.9: Ejemplo de fichero de datos generado por el VNA. 155 


\section{LISTA DE TABLAS}

Tabla 2.1: Requisitos de operación para distintas aplicaciones WBAN [Cordeiro, 2007]. .....10

Tabla 2.2: Comparativa de tecnologías inalámbricas para WBAN.........................................11

Tabla 2.3: Requisitos funcionales del estándar IEEE 802.15.6..............................................14

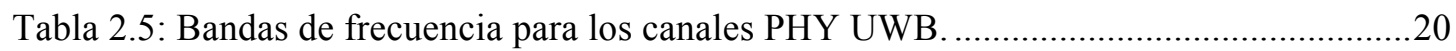

Tabla 2.6: Modulaciones para PHY UWB según los modos de calidad de servicio.................21

Tabla 2.7: Escenarios de interconexión entre dispositivos y modelos de canal [IEEE P802.15-

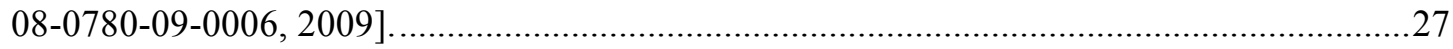

Tabla 3.1: Configuración del VNA para las medidas off-body UWB en estático. ....................44

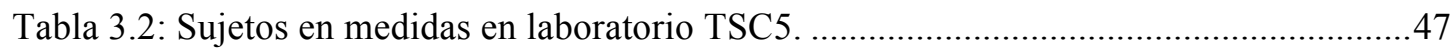

Tabla 3.3: Sujetos en medidas en laboratorio del SiCoMo. ......................................................48

Tabla 3.4: Valores de ajuste al modelo de pérdidas para medidas sin sujetos.........................53

Tabla 3.5: Valores de ajuste al modelo de pérdidas para medidas con sujetos........................53

Tabla 3.6: rms Delay Spread para medidas con y sin sujeto en laboratorios TSC5 y SiCoMo.

Tabla 3.7: Mean Excess Delay para medidas con y sin sujeto en laboratorios TSC5 y

SiCoMo.

Tabla 3.8: Parámetros del modelo de pérdidas para cada enlace off-body Tx-Rx.

Tabla 3.9: Número medio de componentes multicamino recibidas para cada punto de colocación de Rx.

Tabla 3.10: rms delay spread a $30 \mathrm{~dB}$ para las posiciones de Rx sobre el cuerpo de los sujetos a las tres distancias consideradas.

Tabla 3.11. rms delay spread a $30 \mathrm{~dB}$ para las posiciones de $\mathrm{Rx}$ sobre el cuerpo de los sujetos en postura de pie y tumbado.

Tabla 3.12. Número de componentes multicamino recibidas para cada posición de Rx sobre el cuerpo en postura de pie y tumbado. 
Tabla 3.13. Parámetros del modelo de ajuste lineal ...............................................................68

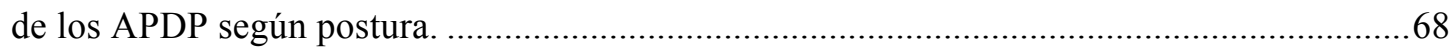

Tabla 3.14. Parámetros obtenidos para el modelo CM4 UWB considerando sujetos de pie y tumbados

Tabla 4.1: Parámetros de configuración del analizador de redes para medidas in-body en condiciones dinámicas.

Tabla 4.2: Valores del modelo teórico de proceso respiratorio para el canal IB2OB..............90

Tabla 4.3: Valores del modelo teórico de proceso respiratorio para el canal IB2OFF. ...........92

Tabla 4.4: Valores del modelo teórico de proceso respiratorio para el canal IB2IB................94

Tabla 4.5: Valor de RMSE para ajuste de la forma del espectro Doppler en el canal IB2OB.98

Tabla 4.6: Valor de RMSE para ajuste de la forma del espectro Doppler en el canal IB2OFF.

Tabla 4.7: Valor de RMSE para ajuste de la forma del espectro Doppler en el canal IB2IB.100

Tabla 4.8: Valores de referencia del Doppler spread para 12 respiraciones/minuto...............102

Tabla 4.9: Parámetros de ensanchamiento Doppler medidos para el canal IB2OB...............103

Tabla 4.10: Parámetros de ensanchamiento Doppler medidos para el canal IB2OFF. ..........106

Tabla 4.11: Parámetros de ensanchamiento Doppler medidos para el canal IB2IB. .............108

Tabla 4.12: Tiempos de coherencia según umbral y tasa de decaimiento de la autocorrelación

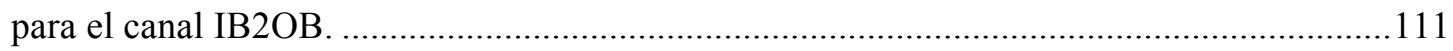

Tabla 4.13: Tiempos de coherencia según umbral y tasa de decaimiento de la autocorrelación para el canal IB2OFF

Tabla 4.14: Tiempos de coherencia según umbral y tasa de decaimiento de la autocorrelación para el canal IB2IB

Tabla B.1: Propiedades dieléctrica de los principales tejidos y órganos a $5 \mathrm{GHz}$. 144 


\section{BIBLIOGRAFÍA}

[Abbasi et al., 2011] Q. H. Abbasi, M. M. Khan, A. Alomainy, Y. Hao, Sectorised Radio Channel Characterisation for Ultra Wideband Body-centric Wireless Communications, Proceedings of the 5th European Conference on Antennas and Propagation (EUCAP), 2011.

[Abbasi et al., 2012] Q. H. Abbasi, M. M. Khan, S. Liaqat, A. Alomainy, Y. Hao, Ultra Wideband Off-Body Radio Channel Characterisation for Different Environments, 7th International Conference on Electrical \& Computer Engineering (ICECE), 2012.

[Ali et al., 2010] A. J. Ali, W. G. Scanlon, S. L. Cotton, Pedestrian Effects in Indoor UWB Off-Body Communication Channels, Loughborough Antennas and Propagation Conference (LAPC), 2010.

[Aoyagi et al., 2008] T. Aoyagi, J. I. Takada, K. Takizawa, N. Katayama, T. Kobayashi, K. Y. Yazdandoost, H. Li, R. Kohno, Channel model for wearable and implantable WBANs, IEEE 802.15-08-0416-04-0006, November 2008.

[Bakar et al., 2011] A. A. Bakar, A. Abbosh, M. Bialkowski, Fabrication and Characterization of a Heterogeneous Breast Phantom for Testing an Ultrawideband Microwave Imaging System, Microwave Conference Proceedings (APMC), Asia-Pacific, December 2011.

[Baskharoun et al., 2012] Y. Baskharoun, A. Trehan, N. K. Nikolova, M. D. Noseworthy, Physical Phantoms for Microwave Imaging of the Breast, IEEE Topical Conference on Biomedical Wireless Technologies, Networks, and Sensing Systems (BioWireleSS), January 2012.

[Begaud, 2011] X. Begaud, Ultra Wide Band Antennas, Ed. John Wiley \& Sons, Ltd, 2011.

[Bello, 1963] P. Bello, Characterization of randomly time-variant linear channels. IEEE Transactions on Communications Systems, vol. 11, no. 4, pp. 360-393, December 1963. 
[Catherwood y Scanlon, 2013] P. A. Catherwood, W. G. Scanlon, Body-centric ultrawideband multi-channel characterisation and spatial diversity in the indoor environment, IET Microwaves, Antennas \& Propagation, vol. 7, no. 1, pp. 61-70, January 2013.

[Chen et al., 2011] X. Chen, X. Lu, D. Jin, L. Su, L. Zeng, Channel Modeling of UWB-Based Wireless Body Area Networks, IEEE International Conference on Communications (ICC), 2011.

[Christ et al, 2010] A. Christ, W. Kainz, E. G. Hahn, K. Honegger, M. Zefferer, E. Neufeld, W. Rascher, R. Janka, W. Bautz, J. Chen, B. Kiefer, P. Schmitt, H. Hollenbach, J. Shen, M. Oberle, D. Szczerba, A. Kam, J. W. Guag, and N. Kuster. The virtual family development of surface based anatomical models of two adults and two children for dosimetric simulations, Physics in Medicine and Biology, vol. 55, no. 2, pp. 23-38, January 2010.

[Cole y Cole, 1941] K. S. Cole, R. H. Cole, Dispersion and absorption in dielectrics: I. Alternating current characteristics, Journal of Chemical Physics, pp.341-351, April 1941.

[Cordeiro, 2007] C. Cordeiro, IEEE 802.15-07-0564-0ban: Use cases, applications, and requirements for bans, January 2007.

[Cotton y Scanlon, 2007] S. L. Cotton, W. G. Scanlon, Higher order statistics for lognormal small-scale fading in mobile radio channels, IEEE Antennas Wireless Propagation Letters, vol. 6 , pp. 540-543, 2007.

[D’Errico y Ouvry, 2011] R. D'Errico, L. Ouvry, Doppler Characteristics and Correlation Properties of On-Body Channels, Proceedings of the 5th European Conference on Antennas and Propagation (EUCAP), April 2011.

[Di Bari et al., 2013] R. Di Bari, Q. H. Abbasi, A. Alomainy, Y. Hao, An Advanced UWB Channel Model for Body-Centric Wireless Networks, Progress In Electromagnetics Research, vol. 136, pp. 79-99, 2013.

[Di Benedetto y Giancola, 2004] M. G. Di Benedetto, G. Giancola, Understanding Ultra Wideband Radio Fundamentals, Ed. Prentice Hall, PTR, NJ, USA, 2004.

[Duncan y Cummings, 2013] K. Duncan, R. E. Cummings, Selecting a Safe Power Level for an Indoor Implanted UWB Wireless Biotelemetry Link, IEEE Biomedical Circuits and Systems Conference (BioCAS), October 2013. 
[Edwards y Khattak, 2010] R. M. Edwards, M. I. Khattak, Understanding Body-centric Antennas, Loughborough Antennas and Propagation Conference (LAPC), 2010.

[ETSI TR 103 181-1, 2015] Short Range Devices (SRD) using Ultra Wide Band (UWB); Technical Report Part 1: UWB signal characteristics and overview CEPT/ECC and EC regulation.

[Floor at al., 2015] P. A. Floor, R. Chavez-Santiago, S. Brovoll,, Ø. Aardal, J. Bergsland, O.J. H. N. Grymyr, P. S. Halvorsen, R. Palomar, D. Plettemeier, S.-E. Hamran, T. A. Ramstad, I. Balasingham, In-Body to On-Body Ultrawideband Propagation Model Derived From Measurements in Living Animals, IEEE Journal Of Biomedical And Health Informatics, vol. 19, no. 3, pp. 938-948, May 2015.

[Fort et al., 2006] A. Fort, J. Ryckaert, C. Desset, P. De Doncker, P. Wambacq, L. Van Biesen, Ultra-Wideband Channel Model for Communication Around the Human Body, IEEE Journal on Selected Areas in Communications, vol. 24, no. 4, pp. 927-933, April 2006.

[Fort et al., 2006b] A. Fort, C. Desset, P. De Doncker, P Wambacq, L. Van Biesen, An UltraWideband Body Area Propagation Channel Model-From Statistics to Implementation, IEEE Transactions on Microwave Theory and Techniques, vol. 54, no. 4, pp. 1820-1826, June 2006.

[Fu et al., 2011] R. Fu, Y. Ye, N. Yang, K. Pahlavan, Doppler Spread Analysis of Human Motions for Body Area Network Applications, IEEE 22nd International Symposium on Personal Indoor and Mobile Radio Communications (PIMRC), September 2011.

[Gabriel y Gabriel, 1996] S. Gabriel, R. W. Lau, C. Gabriel, The dielectric properties of biological tissues: II. Measurements in the frequency range $10 \mathrm{~Hz}$ to $20 \mathrm{GHz}$, Physics in Medicine and Biology, vol. 41, no. 11, pp. 2251-2269, 1996.

[Serna et al., 2015] R. G. G. Serna, C. G. Pardo, J. M. M. Garcia-Pardo, Effect of the Receiver Attachment Position on Ultra Wideband Off-Body Channels, IEEE Antennas and Wireless Propagation Letters, vol. 14, pp. 1101-1104, January 2015.

[Garrett y Kirkendall, 1999] W. E. Garrett, D. T. Kirkendall, Pulmonary Responses to Exercise And Training, in Exercise and Sport Science, Ed. Lippincott Williams and Wilkins, 1999, pp. 117-134. 
[Garrett y Fear, 2015] J. Garrett, E. Fear, A New Breast Phantom With a Durable Skin Layer for Microwave Breast Imaging, IEEE Transactions on Antennas and Propagation, vol. 63, no. 4, pp. 1693-1700, April 2015.

[Ghannoum et al., 2010] H. Ghannoum, C. Roblin, X. Begaud, Investigation and Modeling of the UWB On-Body Propagation Channel, Wireless Personal Communications, January 2010, vol. 52, no. 1, pp. 17-28.

[Ghassemzadeh et al., 2003] S. S. Ghassemzadeh, L. J. Greenstein, A. Kavčić, T. Sveinsson, V. Tarokh, An Empirical Indoor Path Loss Model For Ultra-Wideband Channels, Journal of Communications and Networks, vol. 5, no. 4, pp. 303-308, December 2003.

[Ghavami et al., 2005] M Ghavami, L. B. Michael, R. Kohno, Ultra Wideband Signals And Systems In Communication Engineering, Ed. John Wiley \& Sons, Ltd, 2nd Edition, 2007.

[Goswami et al., 2015] D. Goswami, K. C. Sarma, A. Mahanta, Experimental determination of Path Loss and Delay Dispersion Parameters for On-Body UWB BAN Channel, IEEE International Conference on Signal Processing, Informatics, Communication and Energy Systems (SPICES), 2015.

[Goulianos et al., 2008] A. A. Goulianos, T. W. Brown, S. Stavrou, Ultra-wideband measurement and results for sparse off-body communication channels, Loughborough Antennas and Propagation Conference, Loughborough, UK, March 2008.

[Goulianos et al., 2010] A. A. Goulianos, T. Brown, S. Stavrou, Power delay profile modelling of the ultra wideband off-body propagation channel, IET Microwaves, Antennas \& Propagation, vol. 4, no, 1, pp. 62-71, January 2010.

[Gupta et al., 2013] S. K. S. Gupta, T. Mukherjee, K. K. Venkatasubramanian, Body Area Networks: Safety, Security, and Sustainability, Ed. Cambridge University Press, 2013.

[Guraliuc et al., 2014] A. R. Guraliuc, M. Zhadobov, O. De Sagazan, R. Sauleau, Solid Phantom for Body-Centric Propagation Measurements at $60 \mathrm{GHz}$, IEEE Transactions on Microwave Theory and Techniques, vol. 62, no. 6, pp. 1373-1380, July 2014.

[Hall y Hao, 2012] P. S. Hall, Y. Hao, Antennas and Propagation for Body-Centric Wireless Communications, 2nd Edition, Ed. Artech House, 2012. 
[Hanssens et al., 2016] B. Hanssens, E. Tanghe, L. Martens, C. Oestges, W. Joseph, Measurement-Based Analysis of Delay-Doppler Characteristics in an Indoor Environment, IEEE Transactions on Antennas and Propagation, vol. 64, no. 1, pp. 370-374, January 2016.

[Hirose et al., 2012] M. Hirose, H. Yamamoto, T. Kobayashi, Statistical Modeling of OnBody Ultra-Wideband Channels Considering Surrounding Environments, International Symposium on Wireless Communication Systems (ISWCS), 2012.

[Hoeckel et al., 2015] A. Hoeckel, G. Lee, E. Forrister, D. Xue, G. Benton, B. Garner, Y. Li, "Simulation and Measurement of Dynamic On-Body Wave Propagations", Symposium on Wireless and Microwave Circuits and Systems (WMCS), Texas, USA, April 2015.

[IEEE 802.15.4, 2004] A. F. Molisch, K. Balakrishnan, D. Cassioli, C.-C. Chong, S. Emami, A. Fort, J. Karedal, J. Kunisch, H. Schantz, U. Schuster, K. Siwiak, IEEE 802.15.4a channel model - final report, November 2004.

[IEEE P802.15-08-0780-09-0006, 2009] K. Y. Yazdandoost and K. Sayrafian-Pour, Channel Model for Body Area Network (BAN). IEEE P802.15-08-0780-09-0006, April, 2009.

[IEEE 802.15.6, 2012] 802.15.6-2012 IEEE Standard for local and metropolitan area networks - Part 15.6: Wireless Body Area Networks.

[ICNIRP, 2009] ICNIRP, Exposure to high frequency electromagnetic fields, biological effects and health consequences (100 kHz-300 GHz), 2009.

[IFAC-CNR] An Internet resource for the calculation of the Dielectric Properties of Body Tissues in the frequency range $10 \mathrm{~Hz}-100 \mathrm{GHz}$, http://niremf.ifac.cnr.it/tissprop/.

[Ishido et al., 2004] R. Ishido, T. Onishi, K. Saito, S. Uebayashi, K. Ito, A study on the solid phantoms for 3-6 GHz and evaluation of SAR distributions based on the thermographic method, Proceedings of the 2004 International Symposium on Electromagnetic Compatibility EMC'04, Sendai, Japan, vol. 2, pp. 577-580, June 2004.

[IT'IS Foundation] Virtual Population, https://www.itis.ethz.ch/virtual-population/virtualpopulation.

[Khaleghi et al., 2010] A. Khaleghi, R. C. Santiago, I. Balasingham, Ultra-wideband pulsebased data communications for medical implants, IET Communications, vol. 4, no. 15, pp. 1889-1897, October 2010. 
[Khaleghi et al., 2011] A. Khaleghi, R. Chávez-Santiago, I. Balasingham, Ultra-wideband statistical propagation channel model for implant sensors in the human chest, IET Microwaves, Antennas \& Propagation, vol. 5, no. 15, pp. 1805-1812, December 2011.

[Khaleghi et al., 2012] A. Khaleghi, R. C. Santiago, I. Balasingham, An improved ultra wideband channel model including the frequency-dependent attenuation for in-body communications, Annual International Conference of the IEEE Engineering in Medicine and Biology Society (EMBC), September 2012.

[Khan et al., 2011] M. M. Khan, Q. H. Abbasi, A. Alomainy, Y. Hao, Study of Line of Sight (LOS) and None Line of Sight (NLOS) Ultra Wideband Off-Body Radio Propagation for Body Centric Wireless Communications in Indoor, Proceedings of the 5th European Conference on Antennas and Propagation (EUCAP), 2011.

[Khan et al., 2013] M. M. Khan, Q. H. Abbasi, A. Alomainy, Y. Hao, C. Parini, Experimental characterisation of ultra-wideband off-body radio channels considering antenna effects, IET Microwaves, Antennas \& Propagation, vol. 7, no. 5, pp. 370-380, April 2013.

[Kumar, 2015] S. Kumar, Wireless Signal Propagation and Fading, in Wireless Communications Fundamental \& Advanced Concepts: Design Planning and Applications, Ed. River Publishers, 2015, pp. 127-209.

[Liu et al., 2013] L. Liu, S. van Roy, F. Quitin, P. De Doncker, C. Oestges, Statistical Characterization and Modeling of Doppler Spectrum in Dynamic On-Body Channels, IEEE Antennas and Wireless Propagation Letters, vol. 12, pp. 186-189, February 2013.

[Maduranga et al., 2014] K. Maduranga, S. Thotahewa, J. Redouté, M. Rasit Yuce, Ultra Wideband Wireless Body Area Networks, Ed. Springer, 2014.

[Maman et al., 2014] M. Maman, B. Denis, R. D’Errico, Research Trends in Wireless Body Area Networks: From On-Body to Body-to-Body Cooperation, 8th International Symposium on Medical Information and Communication Technology (ISMICT), 2014.

[Mobashsher y Abbosh, 2015] A. T. Mobashsher, A. M. Abbosh, Artificial Human Phantoms: Human Proxy in Testing Microwave Apparatuses That Have Electromagnetic Interaction with the Human Body, IEEE Microwave Magazine, vol. 16, no. 6, pp. 42-62, July 2015.

[Molisch, 2009] A. F. Molisch, Ultra-Wide-Band Propagation Channels, Proceedings of the IEEE, vol. 97, no. 2, pp. 353-371, February 2009. 
[Movassaghi et al., 2014] S. Movassaghi, M. Abolhasan, J. Lipman, D. Smith, A. Jamalipour, Wireless Body Area Networks: A Survey, IEEE Communications Surveys \& Tutorials, vol. 16, no. 3, pp. 1658-1686, Third Quarter 2014.

[Negra et al., 2016] R. Negra, I. Jemili, A. Belghith, Wireless Body Area Networks: Applications and technologies, Procedia Computer Science, vol. 83, pp. 1274-1281, 2016.

[Oestges, 2015] C. Oestges, Channel Dynamics in Body Area Networks: Recent Results and Challenges, 49th Asilomar Conference on Signals, Systems and Computers, November 2015.

[Olsén et al. 2011] M. F. Olsén, H. Lindstrand, J. L. Broberg, E. Westerdahl, Measuring chest expansion; A study comparing two different instructions, Advances in Physiotherapy, vol. 13, no. 3, pp. 128-132, August 2011.

[OMS, 2015] Organización Mundial de la Salud, Informe Mundial sobre el envejecimiento y la salud, 2015.

[Pahlavan y Levesque, 2005] K. Pahlavan, A. H. Levesque, Wireless Information Networks, Ed. John Wiley \& Sons, Ltd, 2nd Edition, 2005.

[Palacios et al., 2016] S. C. Palacios, C. G. Pardo, A. F. Leal, N. Cardona, A. V. Lluch, Tailor-Made Tissue Phantoms Based on Acetonitrile Solutions for Microwave Applications up to $18 \mathrm{GHz}$, IEEE Transactions on Microwave Theory and Techniques, vol. 64, no. 11, pp. 3987-3994, November 2016.

[Pasquero y D’Errico, 2016] O. P. Pasquero, R. D’Errico, A Spatial Model of the UWB OffBody Channel in Indoor Environments, IEEE Transactions on Antennas and Propagation, vol. 64, no. 9, pp. 3981-3989, September 2016.

[Petrillo et al., 2015] L. Petrillo, T. Mavridis, J. Sarrazin, A. Benlarbi-Delai, P. De Doncker, Statistical On-Body Measurement Results at $60 \mathrm{GHz}$. IEEE Transactions on Antennas and Propagation, vol. 63, no. 1, pp. 400-403, January 2015.

[Rappaport, 1996] T. S Rappaport, Wireless Communication Principle and Practice, Ed. Prentice Hall. 1996.

[Roblin, 2011] C. Roblin, Analysis of the Channel Power Delay Profile of WBAN Scenarios in Various Indoor Environments, IEEE International Conference on Ultra-Wideband (ICUWB), 2011. 
[Saleh y Valenzuela, 1987] A. A. M Saleh, R. A. Valenzuela, A statistical model for indoor multipath propagation, IEEE Journal on Selected Areas in Communications, vol. 5, pp. 128137, February 1987.

[Santiago y Balasingham, 2013] R. C. Santiago, I. Balasingham, Perspectives and Challenges for the Ultra Wideband Capsule Endoscope, COST IC1004 TD(13) 08052, September 2013.

[Santiago y Balasingham, 2013b] R. C. Santiago, I. Balasingham, Computation of the Transmission Frequency Band for the Ultra Wideband Capsule Endoscope, 7th International Symposium on Medical Information and Communication Technology (ISMICT), March 2013.

[Santiago et al., 2013c] R. C. Santiago, K. S. Pour, A. Khaleghi, K. Takizawa, J. Wang, I. Balasingham, H.-B. Li, Propagation models for IEEE 802.15.6 standardization of implant communication in body area networks, IEEE Communications Magazine, vol. 51, no. 8, pp. 80-87, August 2013.

[Santiago et al., 2014] R. C. Santiago, C. G. Pardo, A. F. Leal, A. V. Lluch, I. Balasingham, N. Cardona, Ultra Wideband Propagation for In-Body to In-Body Communications, COST $259 \mathrm{TD}(14)$ 11060, September 2014.

[Sawada et al., 2008] H. Sawada, T. Aoyagi, J. I. Takada, K. Y. Yazdandoost, R. Kohno, Channel model between body surface and wireless access point for UWB band, IEEE 802.1508-0576-00-0006, August 2008.

[See et al., 2012] T. S. P. See, T. M. Chiam, M. C. K. Ho, M. R. Yuce, Experimental Study on the Dependence of Antenna Type and Polarization on the Link Reliability in On-Body UWB Systems, IEEE Transactions on Antennas and Propagation, vol. 60, no. 11, pp. 53735380, November 2012.

[Shechter et al., 2004] G. Shechter, C. Ozturk, J. R. Resar, E. R. McVeigh, Respiratory motion of the heart from free breathing coronary angiograms, IEEE Transactions on Medical Imaging, vol. 23, no. 8, pp. 1046-1056, August 2004.

[Smith, 1999] S. W. Smith, Moving Average Filters, in Digital Signal Processing: A Practical Guide for Engineers and Scientists, 2nd ed., Ed. Newnes, 1999, pp. 277-284. 
[Smith et al., 2009] D. B. Smith, J. Zhang, L. W. Hanlen, D. Miniutti, D. Rodda, B. Gilbert, Temporal correlation of dynamic on-body area radio channel, Electronics Letters, vol. 45, no. 24, pp. 1212-1213, November 2009.

[Smith et al., 2013] D. B. Smith, D. Miniutti, T. A. Lamahewa,L. W. Hanlen, Propagation Models for Body Area Networks: A Survey and New Outlook, IEEE Antennas and Propagation Magazine, vol. 55, no. 5, pp. 97-117, October 2013.

[Støa et al., 2010] S. Støa, R. C. Santiago, I. Balasingham, An ultra wideband communication channel model for the human abdominal region, IEEE GLOBECOM Workshops (GC Wkshps), December 2010.

[Swaisaenyakorn et al., 2012] S. Swaisaenyakorn, P.R. Young, S.W. Kelly, J.C. Batchelor, Comparison of 3D Scanned Human Models for Off-Body Communications using Motion Capture, Loughborough Antennas and Propagation Conference (LAPC), November 2012.

[Takizawa et al., 2008] K. Takizawa, T. Aoyagi, J. Takada, N. Katayama, K. Yekeh, Y. Takehiko, K. R. Kohno, Channel models for wireless body area networks, 30th Annual International Conference of the IEEE Engineering in Medicine and Biology Society, 2008. EMBS 2008.

[Taparugssanagorn et al., 2009] A. Taparugssanagorn, C. Pomalaza-Ráez, A. Isola, R. Tesi, M. Hämäläinen, J. Iinatti, UWB Channel Modeling for Wireless Body Area Networks in Medical Applications, Proc. international symposium on medical information and communication technology (ISMICT), 2009.

[Taparugssanagorn et al., 2009b] A. Taparugssanagorn,C. Pomalaza-Ráez, R. Tesi, A. Isola, M. Hämäläinen, J. Iinatti, UWB Channel for Wireless Body Area Networks at Hospital, 12th International Symposium on Wireless Personal Multimedia Communications WPMC, Sep 710, 2009, Sendai, Japan.

[TG6 TRD, 2008] Zhen B., Patel M., Lee S., Won E., Astrin A. Project IEEE P802.15 Working Group for Wireless Personal Area Networks (WPANs). IEEE Standard Association; Piscataway, NJ, USA: 2008. TG6 Technical Requirements Document (TRD).

[Thotahewa et al., 2015] K. M. S. Thotahewa, J.-M. Redoutè, M. Rasit Yuce, Propagation, Power Absorption, and Temperature Analysis of UWB Wireless Capsule Endoscopy Devices Operating in the Human Body, IEEE Transactions on Microwave Theory and Techniques, vol. 63, no. 11, pp. 3823-3833, November 2015. 
[Toennies et al., 2010] J. L. Toennies, G. Tortora, M. Simi, P. Valdastri, R. J. Webster, Swallowable medical devices for diagnosis and surgery: the state of the art, Journal of Mechanical Engineering Science, vol. 224, no. 7, pp. 1397-1414, July 2010.

[Tuovinen et al., 2013] T. Tuovinen, T. Kumpuniemi, M. Hämäläinen, K. Yekeh Yazdandoost, J. Iinatti, Effect of the Antenna-Body Distance on the On-Extand On-On Channel Link Path Gain in UWB WBAN Applications, 35th Annual International Conference of the IEEE Engineering in Medicine and Biology Society (EMBC), 2013.

[Ullah et al., 2013] S. Ullah, M. Mohaisen, M. A. Alnuem, A Review of IEEE 802.15.6 MAC, PHY, and Security Specifications, International Journal of Distributed Sensor Networks, April 2013.

[UN, 2015] United Nations, Department of Economic and Social Affairs, Population Division (2015). World Population Prospects: The 2015 Revision, Key Findings and Advance Tables. Working Paper No. ESA/P/WP.241.

[Wang y Wang, 2013] J. Wang, Q. Wang, Body Area Communications: Channel Modeling, Communication Systems, and EMC, Ed. John Wiley \& Sons, 2013.

[West, 2012] J. B. West, Mechanics of Breathing, in Respiratory Physiology: The Essentials, 9th ed., Ed. Lippincott Williams \& Wilkins, 2012, pp. 95-124.

[Yilmaz et al., 2014] T. Yilmaz, R. Foster, Y. Hao, Broadband Tissue Mimicking Phantoms and a Patch Resonator for Evaluating Noninvasive Monitoring of Blood Glucose Levels, IEEE Transactions on Antennas and Propagation, vol. 62, no. 6, pp. 3064-3075, June 2014.

[Yoo y Cotton, 2015] S. K. Yoo, S. L. Cotton, A Statistical Characterization of Shadowed Fading in Indoor Off-Body Communications Channels at $5.8 \mathrm{GHz}$, 9th European Conference on Antennas and Propagation (EuCAP), April 2015.

[Yu et al., 2016] Y. Yu, P. F. Cui, W. J. Lu, Y. Liu, H.-B. Zhu, Off-Body Radio Channel Impulse Response Model Under Hospital Environment: Measurement and Modeling, IEEE Communications Letters, vol. 20, no. 11, pp. 2332-2335, November 2016.

[Zasowski et al., 2005] T. Zasowski, G. Meyer, F. Althaus, A. Wittneben, Propagation Effects in UWB Body Area Networks, IEEE International Conference on Ultra-Wideband ICU 2005. 2005. 
[Zhou et al., 2006] J. Zhou, D. Hara, T. Kobayashi, Development of ultra wideband electromagnetic phantoms for antennas and propagation studies, First European Conference on Antennas and Propagation, Nice, France, Nov. 6-10, pp. 1-6, 2006. 


\section{ACRÓNIMOS}

ACF

AFD

AP

APDP

AR

BR

EAP

ECC

EIRP

ETSI

FEM

FDTD

CAP

CE

CEPT

CM

CSA

CW

DAA

FCC

FCS

BER

GTK

HBC

IEEE

IFT

ISM

ITS

LCR
Autocorrelation Function

Average Fading Duration

Access Point

Averaged Power Delay Profile

Autoregressive

Breathing Rate

Exclusive Acces Phase

Electronic Communications Commitee

Equivalent Isotropic Radiated Power

European Telecommunications Standards Institute

Finite Elements Method

Finite Difference Time Domain

Contention Access Phase

Comisión Europea

Conférence Européenne des Postes et Télécommunications

Channel Model

Clear Channel Assessment

Continuous Wave

Detection And Avoidance

Federal Communications Commision

Frame Check Sequence

Bit Error Rate

Group Temporal Key

Human Body Communications

Institute of Electrical and Electronics Engineers

Inverse Fourier Transform

Industrial, Scientific and Medical

Intelligent Transport Systems

Level Crossing Rate 
LDC

LE

LOS

MA

MAC

MAP

MBAN

MICS

MK

MoM

MPC

NB

NLOS

PAN

PDP

PL

PHR

PLCP

PPDU

PS

PSD

PSDU

PTK

QoS

RAP

RMSE

SAR

SHR

RI

RSS

$\mathbf{R x}$

SFD

TG

Tx

US

UWB
Low Duty Cycle

Low Energy

Line of Sight

Moving Average

Media Access Control

Managed Access Phase

Medical Body Area Networks

Medical Implants Communication Services

Master Key

Method of Moments

Multipath Component

Narrow Band

Non Line of Sight

Personal Area Network

Power Delay Profile

Path Loss

Physical-layer Header

Physical Layer Convergence Protocol

Physical Layer Protocol Data Unit

Personal Server

Power Spectral Density

Physical-layer Service Data Unit

Pairwise Temporal Key

Quality of Service

Random Access Phase

Root Mean Square Error

Specific Absortion Rate

Synchronization Header

Rate Indicator

Received Signal Strength

Receptor

Start Frame Delimiter

Task Group

Transmisor

Uncorrelated Scattering

Ultra Wideband 
VNA

WBAN

WCE

WLAN

WMTS

WSS

WSSUS
Vector Network Analyzer

Wireless Body Area Network

Wireless Capsule Endoscopy

Wireless Local Area Network

Wireless Medical Telemetry System

Wide-Sense Stationary

Wide-Sense Stationary Uncorrelated Scattering 